\title{
TRANSITIONING PEER CONSULTING: A TECHNOLOGY-IN-PRACTICE APPROACH
}

By

Julie Abbott Watson

A thesis submitted to Victoria University of Wellington in fulfilment of the requirements for the degree of

Doctor of Philosophy in Information Systems

Victoria University of Wellington

2010 


\section{Abstract}

The purpose of this study is to examine the application of ICT to enhance the peer consulting activities of groups of professionals. In this study, peer consulting is defined as the sharing of people's experience through action and reflection in the context of actual practice (Eisen, 2001). The research is undertaken within two New Zealand counselling services organisations, one for-profit and one not-for-profit. The primary guiding research question is:

"How do NZ social services organisations apply online technologies to enhance the professional development of their staff?"

The study is qualitative in nature, and follows the action research methodology. Within one in-depth action research cycle, key participants of each organisation and the researcher collaborate to describe the problem situation, and select and set up pilot online systems. Groups of counselling practitioners then participate in actual online peer consulting sessions, after which the outcomes of the sessions are evaluated and learnings gained.

Data gathered through interviews, observations and systems statistics are analysed to derive the first of two major theoretical contributions of this research, the Model of Peer Consulting Transition. This model reflects the experiences of the research participants as they move through the developmental stages of Defining, Structuring, Experimenting, Engaging and Embedding.

The second theoretical contribution of this study is the novel application of the Technology-in-Practice framework developed by Orlikowski (2000). This framework is the lens through which the environmental factors that exist within each organisational situation and influence peer consulting transition are explained.

The theoretical models developed in this study provide an important contribution to the use of ICT in facilitating professional development. In addition, the participant organisations benefitted directly from being part of the study. The development of an alternative way to engage staff in professional development activities saves time and financial resources, and engaging in actual peer consulting sessions offered participants the opportunity to further develop their respective professional capabilities. 


\section{Acknowledgements}

“Action Precedes Clarity” (Peter Sheahan, 2007)

Beginning several years ago with a distant research goal and much enthusiasm I could not have predicted the outcomes of this study, or the many life events I would encounter during this time. The pursuit of this $\mathrm{PhD}$ was, at times, an isolating, frustrating, challenging and wholly wonderful experience. Along the way many people have provided me with support and guidance who I wish to thank.

This study was possible because of the involvement of two participant organisations. I appreciate their willingness to engage in this study and share their organisational and personal experiences with me, and their unwavering commitment to the goals of this project. I am also grateful to the individual participants who extended their trust and openness and gave their time, energy and honest testimonies without question.

My supervisor, Professor Pak Yoong, was a constant companion through all stages of this journey. Pak has guided me through the $\mathrm{PhD}$ programme, and his input has been invaluable. Undertaking research in action and working with research organisations to address actual issues meant that the way forward was often changing, and not always clear. I particularly appreciate how Pak encouraged me to have confidence in my thinking, decisions, and actions and challenged me to find clarity of understanding.

I would also like to acknowledge the contributions of my co-supervisor Professor Sid Huff. Sid offered constructive feedback and advice that helped to refine my thinking and focus the thesis.

I am fortunate to have been part of a $\mathrm{PhD}$ study group through which I gained vital support and encouragement. I thank all members for sharing their ideas and experiences with me and for reminding me that although a $\mathrm{PhD}$ pathway is unique, it need not be lonely.

I would also like to thank my friends and family members who listened to countless tales of research anguish, attempted to understand and assist with aspects of my study, and never stopped believing that I would achieve this Doctorate. Special thanks to Mel and Craig Waite for their generous assistance with proofreading and formatting the final thesis.

Lastly, I owe a debt of gratitude to my husband, Ross, for supporting me in every way through the many years that I have undertaken this study. As he recently told a friend, "it's the hardest thing we've ever done". 


\section{Contents}

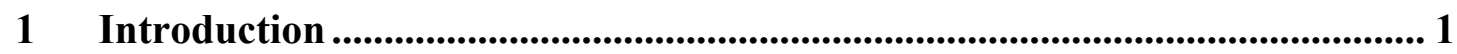

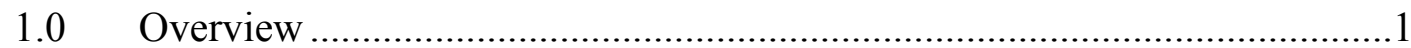

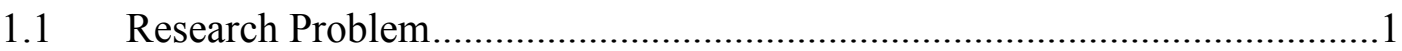

1.2 The Professional Development Challenge ....................................................

1.3 ICT for Continuing Professional Development: nicety or necessity? ............4

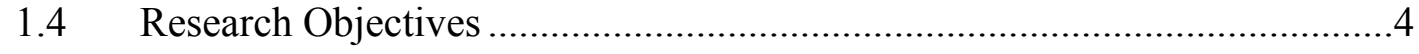

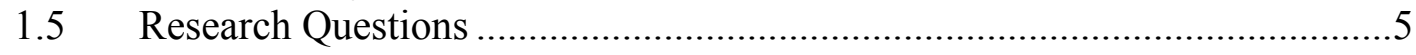

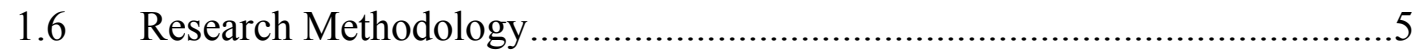

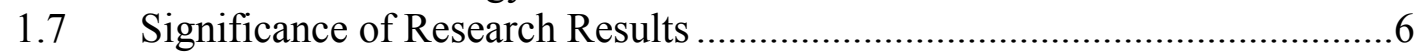

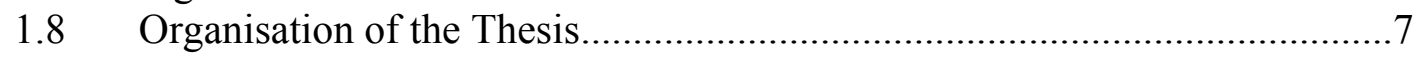

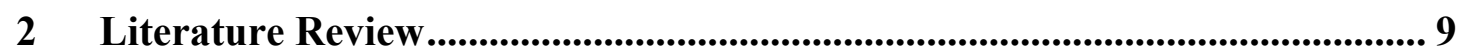

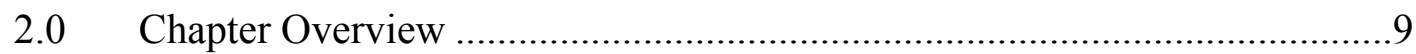

2.1 The Nature of NZ Community Organisations and SMEs .............................9

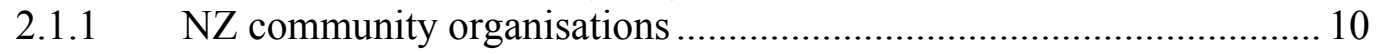

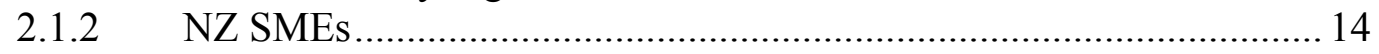

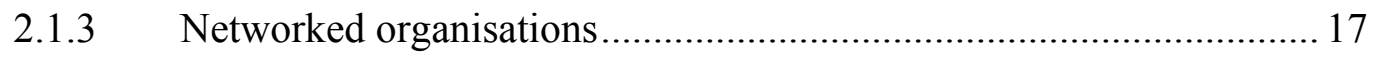

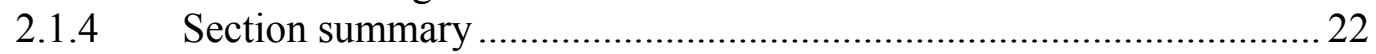

2.2 Technology Support for NZ Community Organisations and SMEs ............23

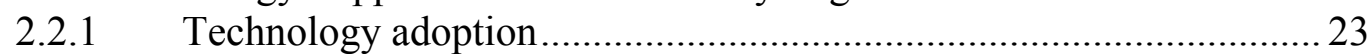

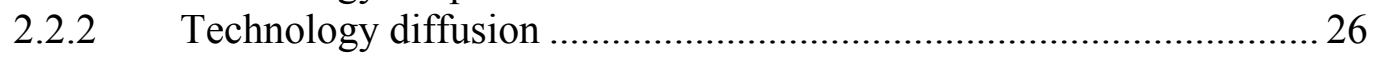

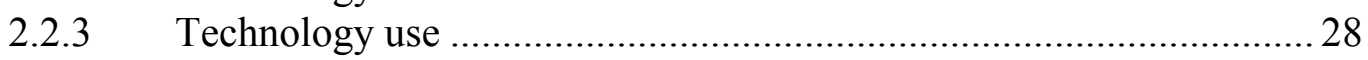

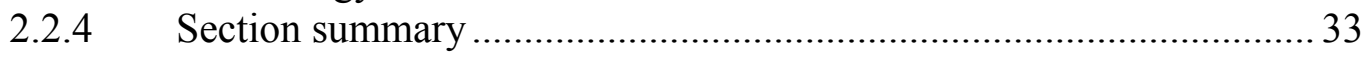

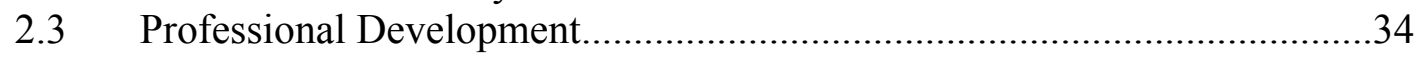

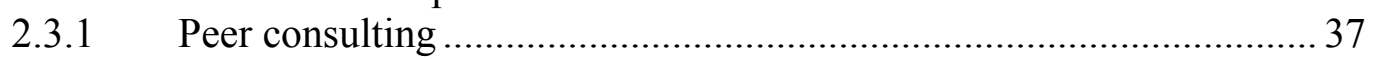

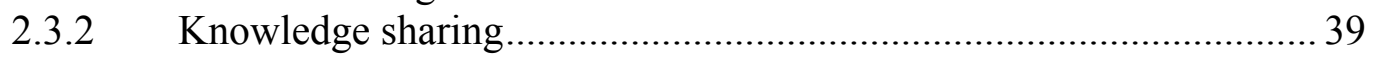

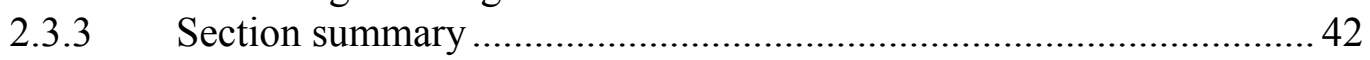

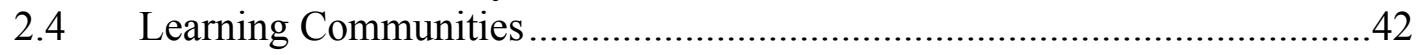

2.4.1 The nature of communities of practice............................................... 43

2.4.2 Technology use within communities of practice.............................. 47

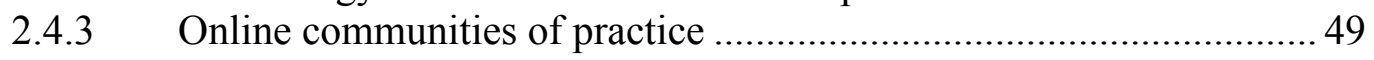

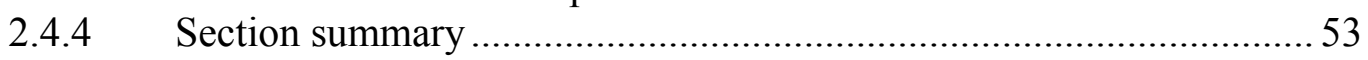

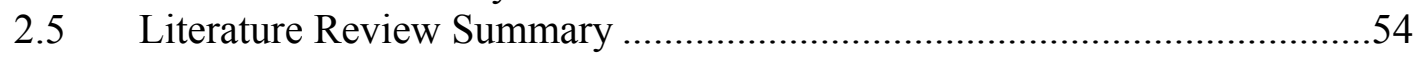

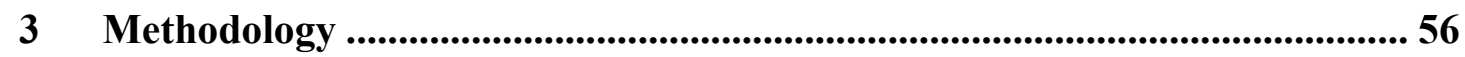

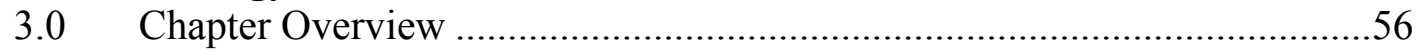

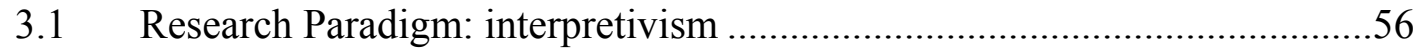

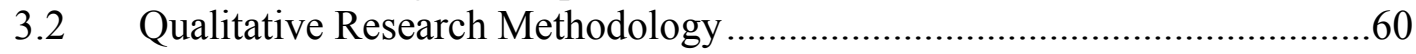

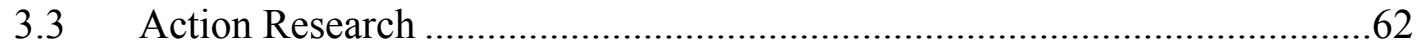

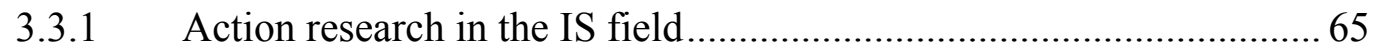

3.4 The Action Research Cycle ..................................................................67

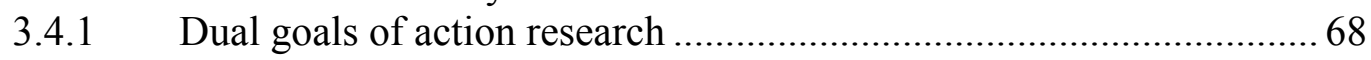

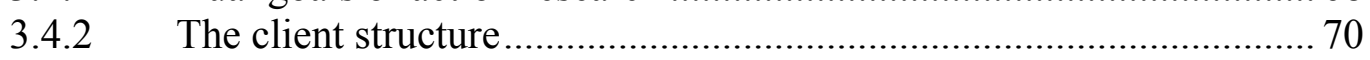

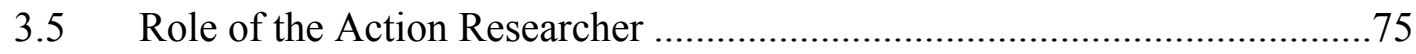

3.5.1 Researcher role at action research stages ....................................... 77 
3.5.2 Collaboration within action research ................................................ 78

3.6 Data Collection Procedures........................................................................ 79

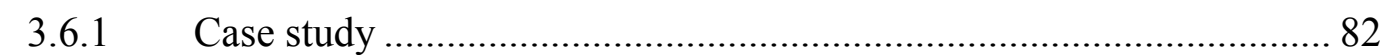

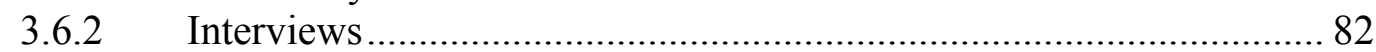

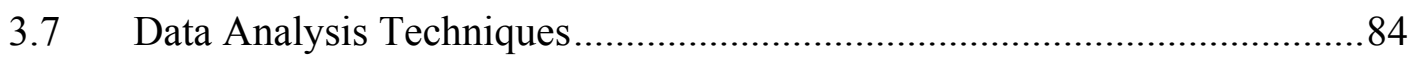

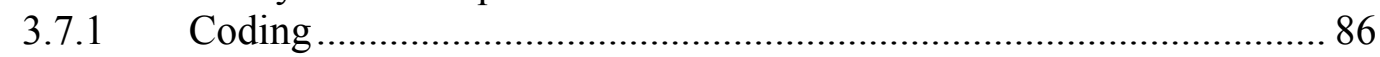

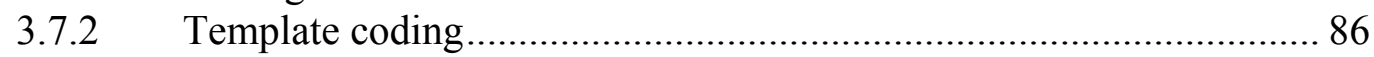

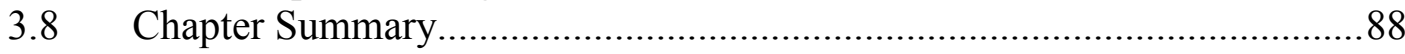

4 Overview of the Model of Peer Consulting Transition ..................................89

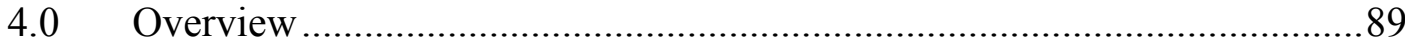

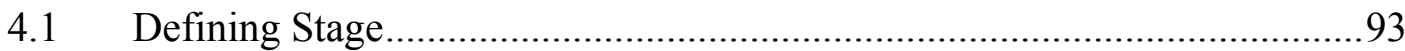

4.1.1 Mapping current peer consulting activities ...................................... 93

4.1.2 Documenting current ICT support ............................................... 94

4.1.3 Developing the online peer consulting concept ............................. 95

4.1.4 Galvanising point ................................................................... 95

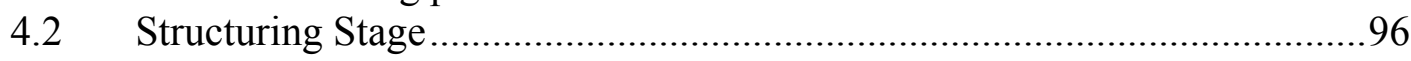

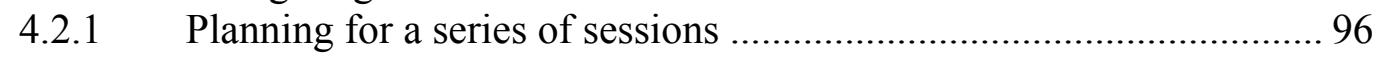

4.2.2 Selecting and setting up systems................................................. 97

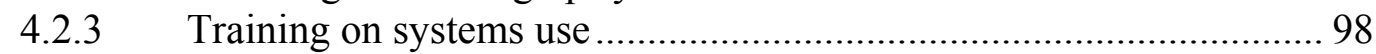

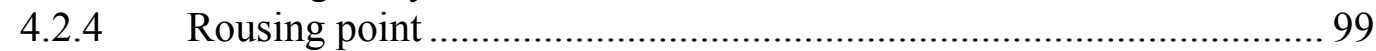

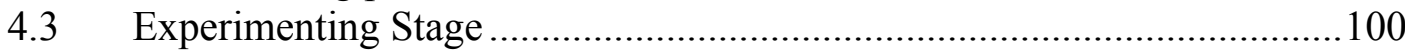

4.3.1 Participating in informal online peer consulting ............................. 100

4.3.2 Altering the plan for sessions ..................................................... 101

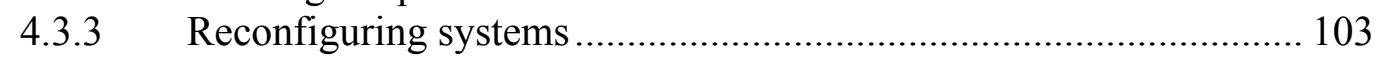

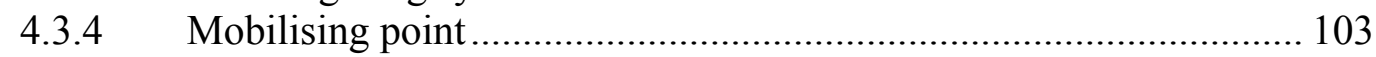

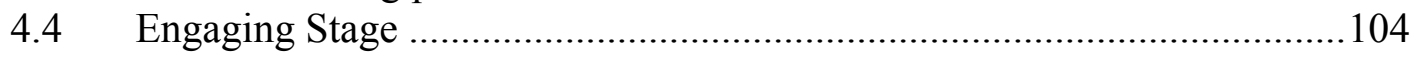

4.4.1 Participating in peer consulting sessions....................................... 104

4.4.2 Formalising peer consulting sessions.......................................... 105

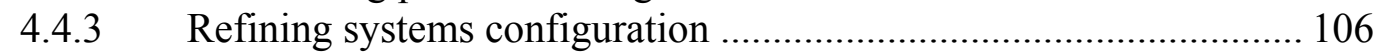

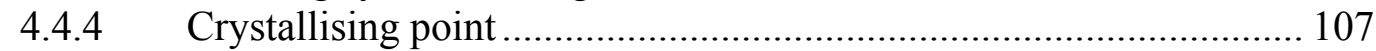

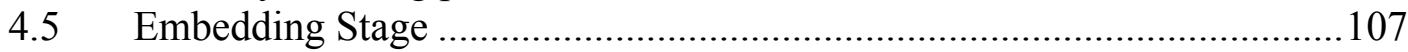

4.5.1 Cementing the online peer consulting concept ............................... 108

4.5.2 Advancing technical infrastructure ............................................... 108

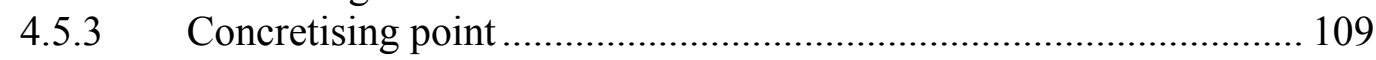

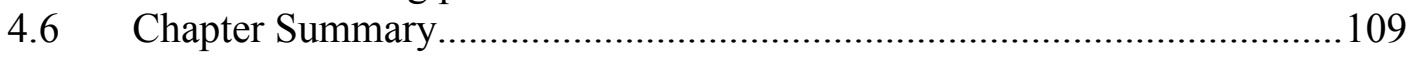

$5 \quad$ Peer Consulting Transition: The Story of Associated Counselling .................89

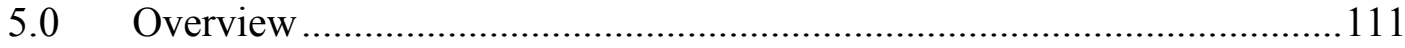

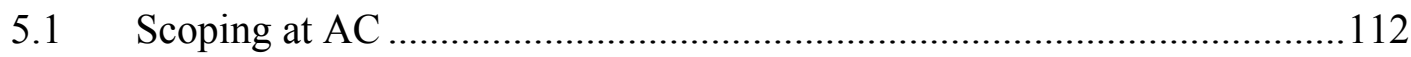

5.1.1 Determining current peer consulting activities ............................ 112

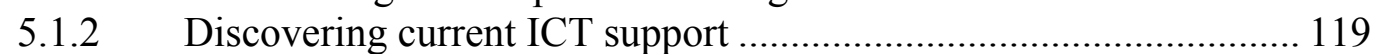

5.1.3 Developing the online peer consulting concept .............................. 120

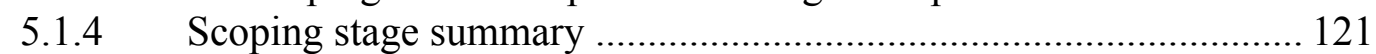

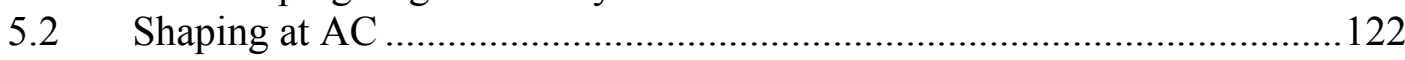

5.2.1 Honing the online peer consulting concept.................................... 123

5.2.2 Selecting and setting up systems.............................................. 128

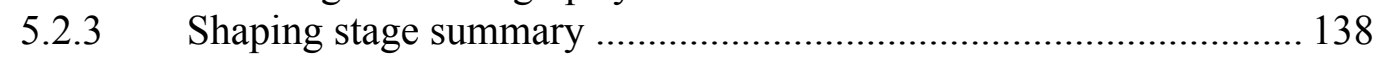

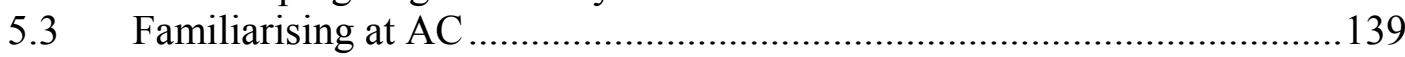

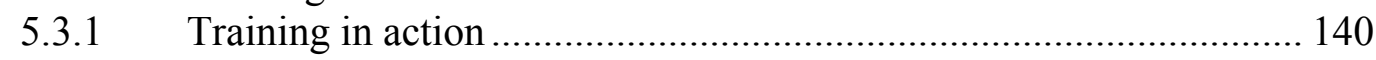


5.3.2 Planning online peer consulting sessions .................................... 142

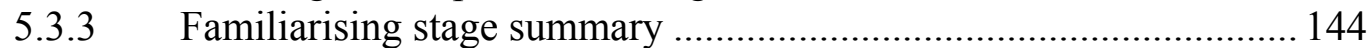

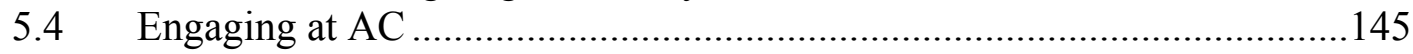

5.4.1 Performing formal online peer consulting .................................... 145

5.4.2 Reforming the online peer consulting sessions ............................. 155

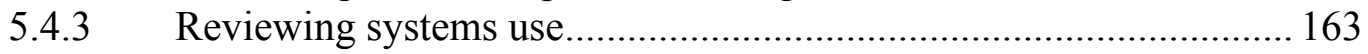

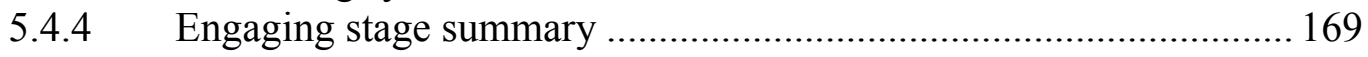

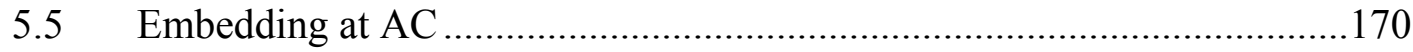

5.5.1 Cementing the online peer consulting concept ............................ 171

5.5.2 Advancing technical infrastructure ............................................ 175

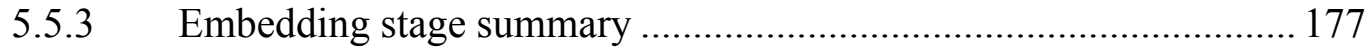

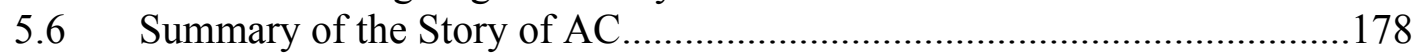

6 Peer Consulting Transition: The Story of Counselling Incorporated ........ 111

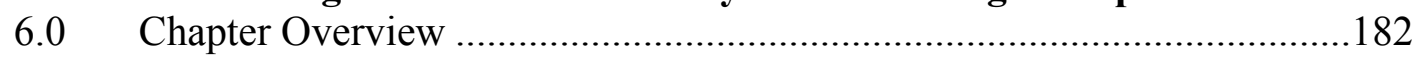

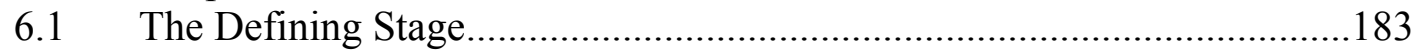

6.1.1 Developing the online peer consulting concept ........................... 183

6.1.2 Mapping current peer consulting activities .................................... 185

6.1.3 Documenting current ICT support ............................................... 191

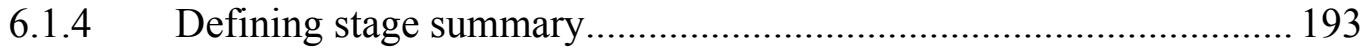

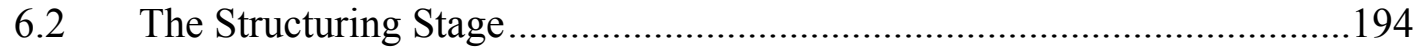

6.2.1 Planning for a series of peer consulting sessions ........................... 195

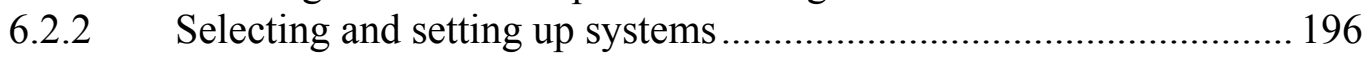

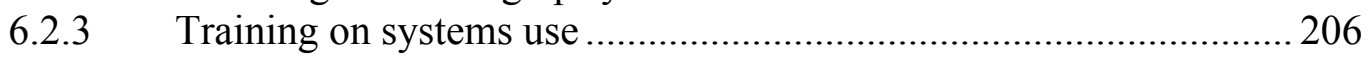

6.2.4 Structuring stage summary ...................................................... 207

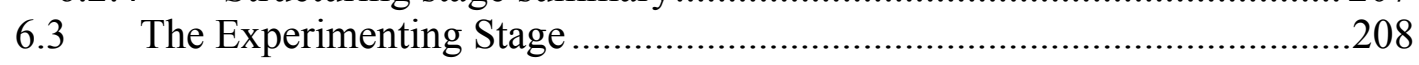

6.3.1 Actioning informal online peer consulting .....................................209

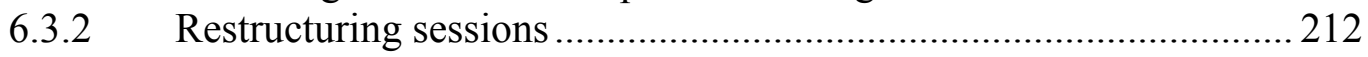

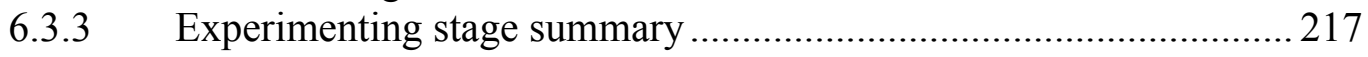

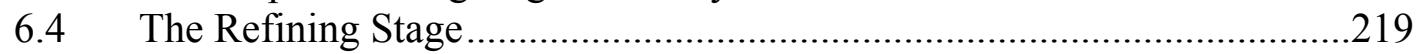

6.4.1 Performing formal online peer consulting .................................... 219

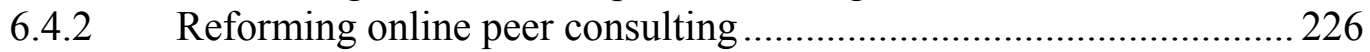

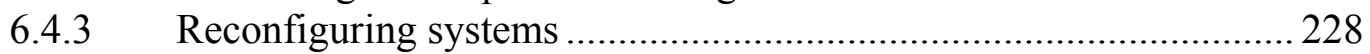

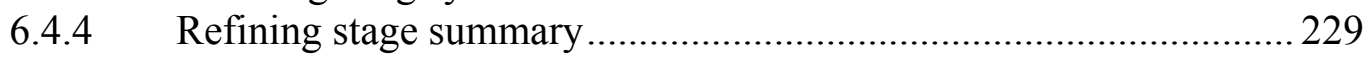

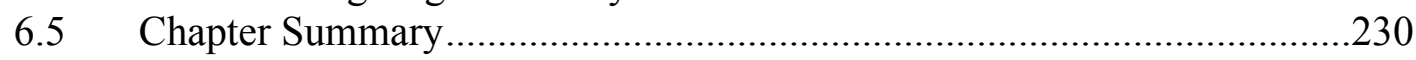

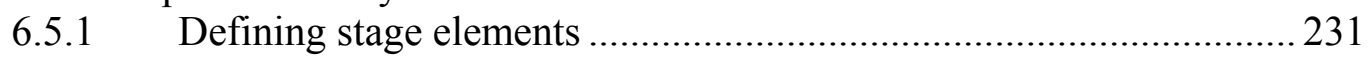

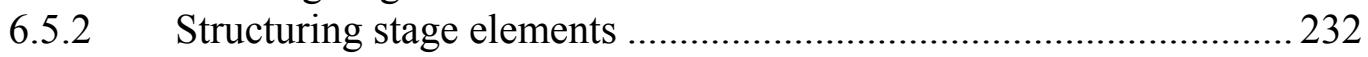

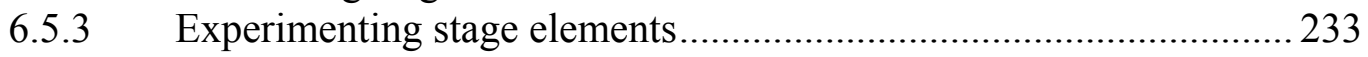

6.5.4 Refining stage elements ........................................................ 233

\section{Enacted Social and Technology Structures: ICT Use and Organisational}

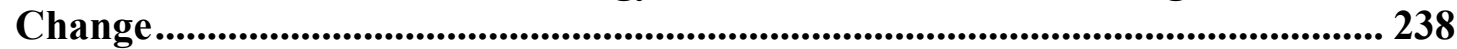

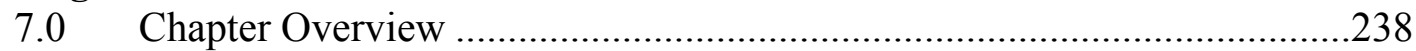

7.1 Technology-in-Practice Framework ......................................................2239

7.2 Application of the Technology-in-Practice Framework............................247

7.3 Technologies-in-Practice at the Engaging Stage......................................249

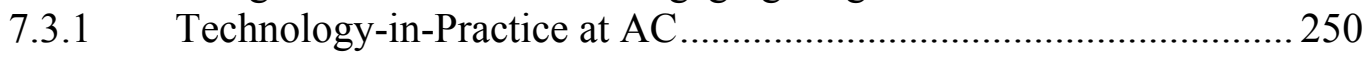

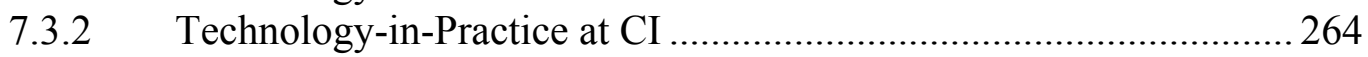

7.4 Technology-in-Practice Summary: Organisational Factors Revealed .......280 
8 Discussion, Implications and Conclusion ......................................................282

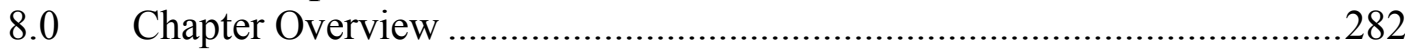

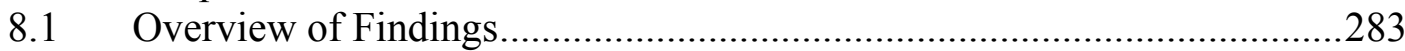

8.2 Addressing the Research Questions .....................................................28

8.2.1 Model of Peer Consulting Transition.............................................. 285

8.2.2 Application of the TiP Framework ............................................... 288

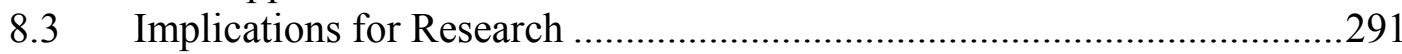

8.3.1 Generating the Model of Peer Consulting Transition ....................... 292

8.3.2 Applying the Technology-in-Practice framework ......................... 293

8.3.3 Applying Media Synchronicity Theory …......................................... 295

8.3.4 Highlighting leadership roles ......................................................... 296

8.3.5 The Distance Action Research approach ........................................ 297

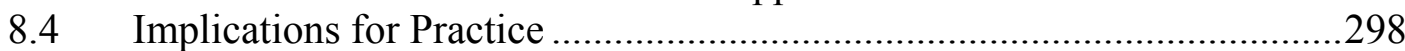

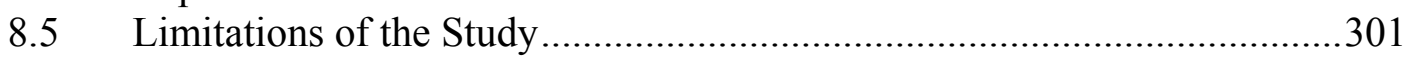

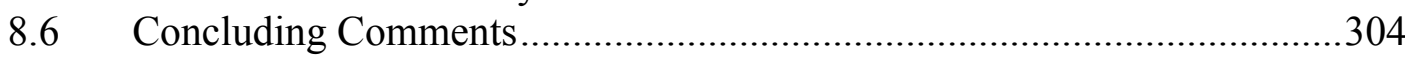

\section{Appendices}

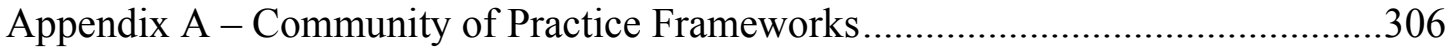

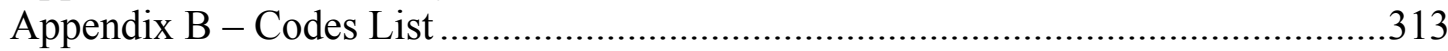

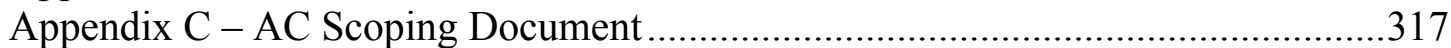

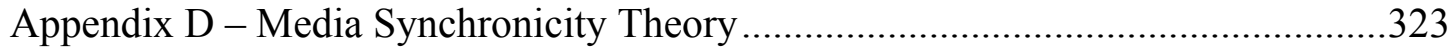

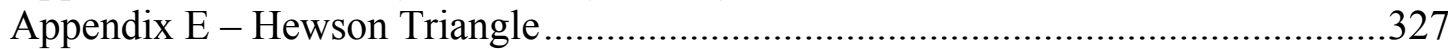

Appendix F - Issues on which CI Peers Consult ..................................................330

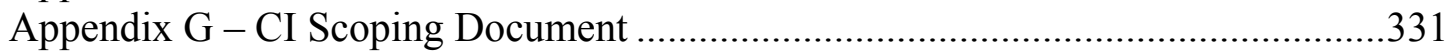

Appendix H - The Nature of CI's Peer Consulting Process ......................................333

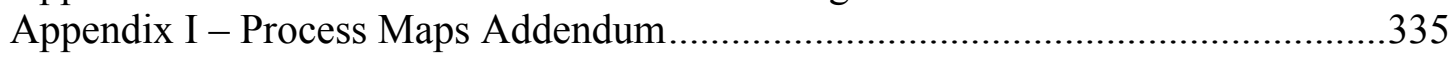

Appendix J - CI Peer Consulting Session Plan .........................................................336

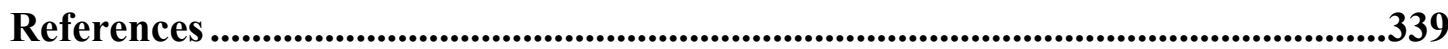

\section{List of Figures}

Figure 1: The Action Research Cycle (Baskerville \& Pries-Heje, 1999; p. 4).......... 68

Figure 2: Action Research Viewed as a Dual Cycle Process (McKay \& Marshall, 1999; p. 603).

Figure 3: Researcher Role in Action Research (Chisholm \& Elden, 1993; p. 287). . 75

Figure 4: Stages of Peer Consulting Transition....

Figure 5: Model of Peer Consulting Transition ..................................................... 110

Figure 6: Elements of Media Synchronicity Theory (adapted from Dennis \&

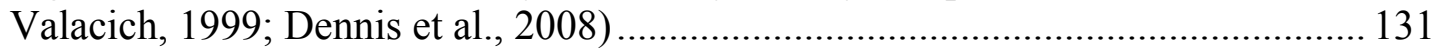

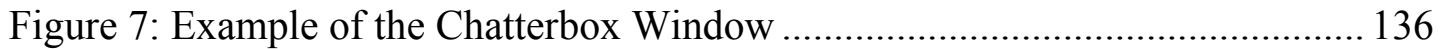

Figure 8: Peer Consutling Transition at AC ...................................................... 180

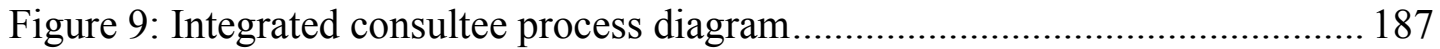

Figure 10: Integrated consultor process diagram ................................................... 189

Figure 11: Integrated Consultee Process Diagram Showing Communications

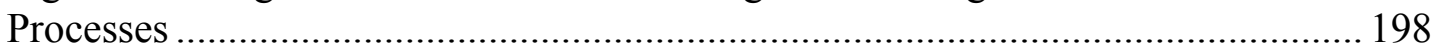

Figure 12: Integrated Consultor Process Diagram Showing Communications

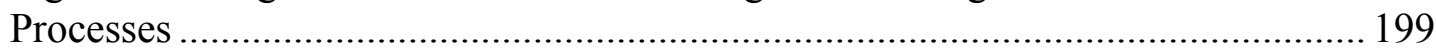

Figure 13: View of a Blackboard Window …....................................................... 204

Figure 14: The Stages of Peer Consulting Transition at CI ................................... 236 
Figure 15: Enactment of Technologies-in-Practice (adapted from Orlikowski, 2000)

Figure 16: Collaborative Technology-in-Practice at the Engaging Stage within

AC

Figure 17: Isolated Use Technology-in-Practice at the Refining Stage within CI... 278

Figure 18: Stages of Development (Wenger, 1998; p. 3)...................................... 307

Figure 19: The Stages of Community Development (McDermott, 2000; p. 17). .... 309

Figure 20: A Supervision Triangle (Hewson, 2002) ............................................... 328

Figure 21: Detail of Hewson Supervision Structure ............................................... 329

\section{List of Tables}

Table 1: Managerial Practices and IS Competencies (Adapted from Cragg, 2008) .. 27

Table 2: The Main Categories of Information Systems (Franke, 2001; p. 54-55).... 32

Table 3: Characteristics of Positivism and Interpretivism (adapted from Weber, 2004;

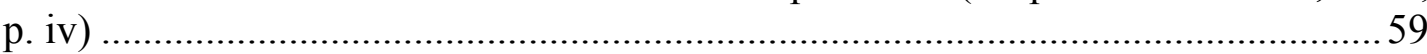

Table 4: Summary of Participant Organisations ..................................................... 71

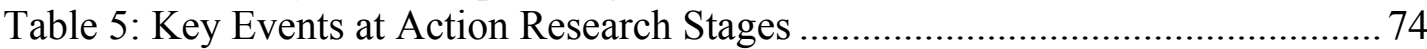

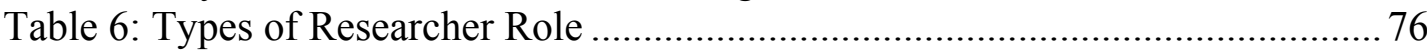

Table 7: Summary of Researcher Role at Action Research Stages...........................77

Table 8: Data Collection Procedures in IS Studies .................................................... 80

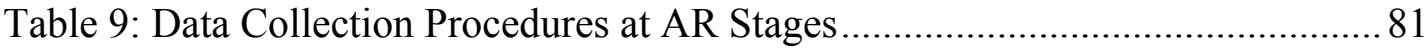

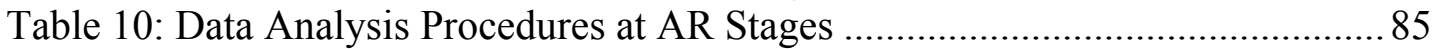

Table 11: Components of AC's Peer Consulting Transition.................................... 179

Table 12: Integrated consultee process diagram elaboration ................................. 188

Table 13: Integrated consultor process diagram elaboration ................................. 190

Table 14: ICT tools used to support peer consulting at CI...................................... 192

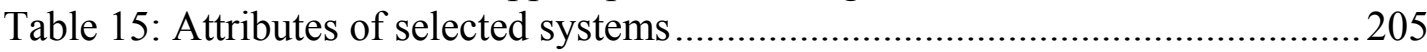

Table 16: Comparison of Defining stage activities ................................................2 231

Table 17: Comparison of Structuring stage Trigger points.................................. 232

Table 18: Comparison of Experimenting stage activities.......................................2233

Table 19: Comparison of Refining stage components.......................................... 235

Table 20: Elements of the Technology-in-Practice Framework (adapted from

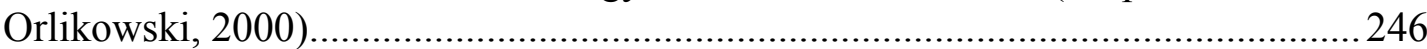

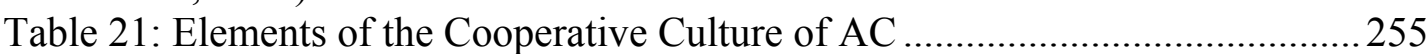

Table 22: Elements of the Flexible Nature of AC.................................................. 258

Table 23: Elements of the Adaptable Communication Structures within AC ......... 260

Table 24: Elements of the Regionally Focused Organisational Structure of CI ......268

Table 25: Elements of the Individualistic Organisational Culture of CI................. 272

Table 26: Elements of the Institutionalised Meeting Structures of CI....................2275

Table 27: Components of Transition Models.......................................................28 287

Table 28: Overview of Key Elements of TiPs at AC and CI ..............................2290

Table 29: Community Evolution Model Definition (Gongla \& Rizzuto, 2001; p. 845).

Table 30: Fundamental Functions for the Stages of Evolution (Gongla \& Rizzuto,

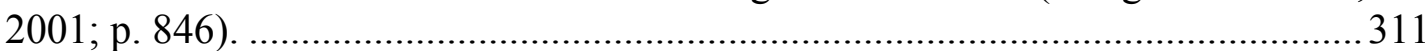

Table 31: Potential Stage Enablers that Promote Connection (Gongla \& Rizzuto,

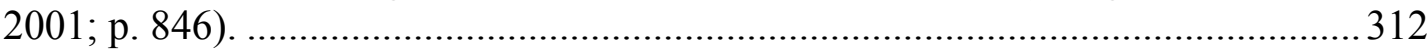

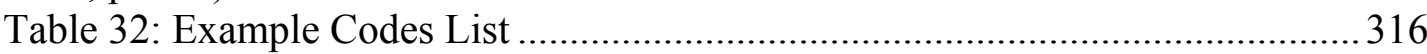

Table 33: Relative Trait Salience of Selected Media (Dennis \& Valacich, 1999; p. 3) 


\section{Introduction}

\subsection{Overview}

The thesis reports research undertaken to explore the provision and use of information and communication technology (ICT) for professional development within New Zealand (NZ) social services (counselling) organisations. The sections of this introductory chapter begin with a description of the nature of the research problem and the challenges such organisations face in providing regular opportunities for staff to engage in continuing professional development. Particular emphasis is placed on the importance of continuing professional development, and the increasing role of ICT in supporting such activities.

Next, the research approach is outlined, starting with the overall research objective of developing theory of how social services organisations go about introducing ICT for professional development. The research questions that guided the study and the Action Research method of pursuing these questions are also provided. Significance of the results of the research to organisational practitioners and academics is also given. Lastly, the introduction concludes by outlining the organisation of the remainder of the thesis.

\subsection{Research Problem}

The NZ economy includes a mix of Government agencies, commercial organisations and a vibrant not-for-profit (N4P) sector. Most prominent in NZ are Small to Medium Sized Enterprises (SMEs), making up 97\% of NZ organisations, 30\% of employment and offering a significant contribution to the economy (Ministry of Economic Development, 2009). Many of these are driven by motives other than profit, termed N4P organisations, and have a long history of actively offering support 
services in the areas of sports, arts, culture and social services to a wide range of people in communities across the length of the country (Thornton, 2010). Examples of N4P organisations within NZ that provide services such as education, counselling and personal development are YMCA, Relationship Services, Barnardos and Plunket Society. These organisations typically include a mix of paid and voluntary staff to deliver their services, and are challenged by relatively high turnover rates that can impact service quality (Robinson, 2001; MED, 2009). For the purposes of this research, SMEs and N4Ps offering support services to NZ communities have been termed 'community organisations'.

Recent changes in the economic and social environments have impacted all sectors of the economy and have particularly impacted the way that community organisations operate, as they often lack the resources, including finance and a permanent staff base, that are needed to support change and have therefore had to be more innovative in their responses (Putnam, 1993). The four most significant issues currently challenging these organisations are:

- Financing their activities

- Fundraising

- Governance

- Retaining/motivating staff (MED, 2009; Thronton, 2010).

Seventy two percent of the N4P organisations surveyed in 2009 reported that financing activities was of most concern, the highest level since 2003 (Thornton, 2010). Nearly a quarter of respondents selected retention and motivation of staff as the most significant issue they face, and in the economic downturn and recovery "there can be significant improvements to be achieved through effective utilisation of both humans and tangible assets" (Thornton, 2010; p. 6). In order to continue to keep pace in today's challenging environment and successfully deliver professional services, community organisations must turn inward and address the competencies of their staff which requires continuing advancement of their practice-related skills and knowledge. This capacity building is termed professional development, as next highlighted.

\footnotetext{
${ }^{1}$ Additional detail of community organisations is provided in Chapter 2.
} 


\subsection{The Professional Development Challenge}

Although many community organisations do not compete in a commercial sense, they must maintain a high level of service quality in order to receive public funding, meet regulations, achieve necessary accreditations and gain client referrals from Government and other agencies (Massey \& Harris, 2007). This continual up-skilling is most often achieved through processes of professional development, defined as "systematic, ongoing, self directed learning" of organisational practitioners (Jones \& Robinson, 1997; p. 198).

While NZ community organisations have recognised the importance of staff professional development the dilemma faced by most, if not all, are inadequate financial resources to provide adequate and ongoing development opportunities. These organisations operate within densely populated main centres and small rural communities, with staff often spread over a wide geographical area, sometimes NZwide. Airfares and accommodation expenses to bring people together for regular face-to-face professional development are beyond the means of most community organisations. Despite this, changes in practice such as best practice stories and new work procedures must still be shared among peers. As Desouza and Awazu argue, "SMEs compete on their know-how and hence have to use knowledge to their advantage, even more so than traditional resources" (p. 33).

Those who join together in groups to share their knowledge to solve practice related problems and learn from each other can be termed a community of practice $(\mathrm{CoP})$, defined as "a collection of individuals bound by informal relationships that share similar work roles and a common context" (Gongola \& Rizzuto, 2001; p. 842). To more firmly ground their activities as professional development I have termed such peer groups 'learning communities', described by Etienne Wenger as "a community in which you can discuss the latest ideas, explore them together and get feedback" (De Cagna, 2001; p.7). Within a learning community these activities are termed 'peer consulting', the sharing of experience among a group of colleagues through action and reflection in the context of actual practice. 
There is little prior research into the use of communities for the provision of professional development in NZ community organisations, and less so in distributed community organisations, (Lewis, Massey, Ashby, Coetzer \& Harris, 2007; Massey $\&$ Harris, 2007). In the distributed environment where staffs are dispersed their professional development activities need to be supported by various ICT, as next described.

\subsection{ICT for Continuing Professional Development: nicety or necessity?}

The provision of social services by community organisations relies heavily on faceto-face interactions, although various ICTs, some simple and some more advanced, are used to carry out their daily work. Due in part to a lack of resources including time, finance and skilled personnel, these organisations have traditionally been slow to adopt and benefit from ICT, particularly online forms such as the Internet, intranets and extranets. However, since 2006 ICT use has been steadily increasing, with the effects of improved responsiveness to customers and greater internal efficiencies (MED, 2009). In the distributed environment, ICTs become a precondition to ongoing professional development, supporting the communications and collaborations needed to develop relationships and enable peer-based learning within communities (Lock, 2006). Without ICTs, distributed staff are likely to remain professionally isolated, without access to the community environment within which many professional development activities take place.

\subsection{Research Objectives}

The main objective of this research was to develop theory on the process of applying ICTs to support the professional development activities of practitioner groups within NZ social services organisations. The study examined particular aspects of this 
process, and mapped out the successful pathway of transitioning peer consulting to the online environment.

A secondary objective was to examine the use of ICTs by a community of learning, more specifically to examine their situated use across time and outline the impacting organisational (and other) factors.

\subsection{Research Questions}

In response to the problem situation outlined above, this study was guided by the primary research question:

“How do NZ social services organisations apply online technologies to enhance the professional development of their staff?"

In order to explore the use of particular ICTs within learning communities a secondary research question was also pursued:

"Within communities of practice, what is the nature of the use of online technologies for professional development activities?"

\subsection{Research Methodology}

A qualitative, Action Research approach was used to pursue the abovementioned research questions. Qualitative methods are most effective in answering such research questions, that are descriptive 'how' and 'why' questions.

Two separate in-depth action research cycles were undertaken that comprised the stages of diagnosing, action planning, action taking, evaluating outcomes and 
specifying learning. Action research does not specify data collection and analysis procedures, and so various techniques were chosen. Interviews were the key data collection procedure applied at each action research stage, complimented by other data forms such as ICT statistics, observations and organisational documentation that offered another perspective throughout the study.

Data organisation and analysis did, at times, occur concurrently with data collection. Analysis techniques followed the coding and pattern matching methods as prescribed by Miles \& Huberman (1984) and Yin (1994) in order to explore data, identify themes and develop categories among and across data sets. Such techniques ensured reliable and rigorous collection and analysis of data and led to valid results, most importantly development of a theory of transitioning professional development to online environments.

\subsection{Significance of Research Results}

This study offers two main contributions to the body of knowledge on the application of ICTs for professional development within learning communities. Firstly, the key stages and activities that NZ community organisations work through when complimenting traditional, face-to-face professional development activities with an online programme have been detailed and are represented as the Model of Peer Consulting Transition (see Chapter 4). Secondly, examining the use of particular ICTs in actual online peer consulting sessions brought to light several organisational factors that acted to inhibit or accelerate the transition process and impacted overall success of online peer consulting initiatives (see Chapter 7).

The findings offer benefits for both research and practice. Firstly, there are several academic contributions. The Model of Peer Consulting Transition sheds light on the actions taken and process followed by NZ SMEs when introducing and applying ICTs for professional development. As Massey et al. (2004) noted, there is a need for research that offers well grounded insights into the operations of SMEs and this 
study contributes significantly to this disjointed body of knowledge. Also, Chapter 7 reports the findings of an in depth analysis of organisational factors that impeded and/or encouraged ongoing ICT use. Extending these findings through replicating the theoretical results in different organisational settings, with different ICT tools or with different professional development processes would provide valuable comparisons and new research knowledge.

Secondly, practitioners may benefit in a number of ways. The participant organisations have benefited from the development of an alternative way to enable groups of staff to engage in professional development. Other organisations now stand to benefit by not only saving time and financial resources, but through experiencing improvements in productivity and service delivery levels due to likely advancements in the skills and knowledge of staff. The third group that will see some advantage from the outcomes of this research are practitioners themselves. In addition to the capacity building that occurred when engaged in professional development, socially and professionally isolated staffs are now able to be part of a community of practitioners, no matter where in NZ they are located. Those in a leadership role in commercial and especially N4P organisations can gain an increased understanding of what constitutes leadership in online ICT provision projects by reviewing the actions of key participants throughout transition. By detailing the activities actually undertaken within two NZ social services organisations, it is hoped that other organisations both within and outside of NZ may gain confidence to begin the process of applying online ICTs to support the professional development activities of their staff.

\subsection{Organisation of the Thesis}

The remainder of this thesis is organised as follows. Chapter 2 summarises the research literature on the subjects of distributed community organisations in NZ, the nature of ICTs that support these organisations, traditional and emerging professional development activities they engage in, communities of practice (as communities of 
learning), and leadership. Chapter 3 gives an explanation of the action research approach of the study, details the data collection and analysis procedures applied and introduces the participant organisations. Chapters 4-8 present the research results; Chapter 4 introduces the key theoretical framework; the Model of Peer Consulting Transition, and Chapters 5 and $\mathbf{6}$ analyse the journey of two participant organisations through this model. Elaborating on the findings of Chapters 4-6, Chapter 7 provides more detailed analysis of ongoing ICT use, and the organisational factors at play that impacted peer consulting transition, and finally Chapter 8 discusses the contribution and implications of the study and concludes the thesis. 


\section{Literature Review}

\subsection{Chapter Overview}

This chapter provides a review of the five key areas of literature that underpin the study and, more specifically, support the research questions explored and results reported. This literature review does not provide an exhaustive examination of key concepts, theories or practices of the study, but instead provides the foundation on which the theory of peer consulting transition was created (see Chapter 4) and the lens through which the stories of the two participant organisations are told (see Chapters 5 and 6).

The chapter is organised into four key sections that: 1) address the nature of community organisations and Small and Medium-sized Enterprises (SMEs), 2) assess the ICTs that support these organisations, 3) explore the nature of professional development, and 4) review the role that communities of practice have to play.

\subsection{The Nature of NZ Community Organisations and SMEs}

Within NZ, a significant number of organisations can be classified as 'community' or 'third sector' organisations. While the discussion below will show that there are a variety of organisational forms and activities, common characteristics are teased out and provide a valuable insight into the nature of $\mathrm{NZ}$ community organisations, and thus also into the organisations that participated in this study.

The remainder of this section introduces the nature of the SME. NZ community organisations share many key attributes with SMEs. Indeed, in NZ 97\% of 
organisations can be classed as SMEs as they employ few staff, are often networked organisations and do not have available the types of professional support and resources of larger organisations.

\subsubsection{NZ community organisations}

Community, or 'social services' organisations in NZ are commonly placed in the public sector, the remaining societal sectors being private and household. Although community and other not-for-profit (N4P) organisations and government are seen as fulfilling complementary roles in the development and delivery of public policy and services, the typical community organisation does not fit well within the public sector. The groups, organisations and networks that are neither government nor business nor household, but have an active and visible role in society can be classed as third sector organisations, the household sector then becoming the fourth sector of society (MSD, 2001).

This third sector is dynamic in nature, with new groups and organisations, both forprofit and N4P, constantly formed to meet newly discerned community needs. The diversity and inherent complexity of this sector means that there is not a single group with a single voice, resulting in a lack of agreed definition of the NZ community sector (MSD, 2001).

Further confusing definitions of this sector, the terms community, voluntary, and N4P organisation are often used interchangeably. To clarify, it is useful to make some distinction between 'voluntary' and 'community'. The term community ${ }^{2}$ is generally used to convey the idea of individuals sharing a network of relationships and forming a common identity either on the basis of a shared locality, common cultural and historical identity or through shared interests (Bruce \& Easley, 2000; MSD, 2001). Community organisations are, therefore, primarily characterised by a sense of mutuality or common interest. In contrast, the use of the term 'voluntary' is

\footnotetext{
${ }^{2}$ The nature of a community is further discussed in section 2.5.
} 
derived from the notion of a lack of coercion or acting from one's own free will (Bussell \& Forbes, 2002). Therefore, relationships within the voluntary sector are based on freedom to choose whether or not to participate. The term voluntary organisation also differs from that of the general community organisation in that it encompasses the idea of 'charity' - helping or caring for those other, usually less fortunate, than oneself (Bruce \& Easley, 2000).

N4P organisations in NZ vary in size and structure. There exist small, primarily volunteer-based initiatives that survive on a few thousand dollars a year, as well as large national organisations that have a corporate structure, hundreds of staff and multi-million dollar budgets (MSD, 2001; Thornton, 2010). They also vary in the types of activities offered to communities, from national social service providers, to informal children's playgroups and neighbourhood networks.

Despite the diversity of organisational forms and activities, there are several characteristics that define third sector organisations:

- A clear sense of values: in particular, independence (freedom of association), altruism, (compassion/ caring for each other - not motivated by profit), trust, cooperation and interdependence, stewardship, hope and passion.

- Purpose: a primary focus on 'doing' the work itself, on contributing to the social good arising from the activity of the organisation, rather than on making a living or profit from it

- Function: fulfilling all or any of the following: advocacy and working for change; mutual aid; developing local solutions to local problems; providing avenues for citizen participation and up-skilling; expressing and fostering culture and identity; and developing networks of goodwill, shared understanding and trust

- Structural or operational: separate from the government, collectively owned and/ or self-governed on the basis of stewardship rather than ownership, and not returning profits to owners or directors (MSD, 2001; p. 41).

Expanding on the structural/operational element listed above, this approach limits the sector to organisations that are: formal, the organisation has some institutional reality; private, the organisation is institutionally separate from the government; non-profit, not returning profits to owners or directors; self-governing, equipped to control their 
own activities; and voluntary, involving some meaningful degree of willing participation, either in the actual conduct of the agency's activities or in the management of it's affairs (p. 40).

Recently in NZ there has been increased government funding available for organisations that provide social services (Lewis et al., 2007). Despite this, many large service providers are experiencing increased financial pressures brought about by the economic downturn, and as they embark on investments in the areas of ICT and staff professional development (MSD, 2001; Thornton, 2010). The need to invest in professional development has arisen out of the realisation that all organisations, whether for- or not-for-profit, need to improve organisational efficiencies and better manage the staffs that deliver social services (MSD, 2001).

The mix of paid and voluntary staff that is associated with a particular community organisation varies. Some operate entirely on unpaid voluntary effort, while other organisations, such as health and social service providers are often large and complex with hundreds of paid staff (MSD, 2001; Bussell \& Forbes, 2002). The reliance on voluntary activity is a key defining feature of many, though not all, organisations in the third sector (Morris, 1999). Volunteering is the commitment of and energy for the benefit of society or the community, simply defined as "unpaid work outside the household sector though organised groups" (MSD, 2001; p. 35). Or more clearly defined as "any activity which involves spending time, unpaid, doing something which aims to benefit someone (individuals or groups) other than or in addition to close relatives, or to benefit the environment" (Morris, 1999; p. 249). While strongly associated with the idea of 'unpaid' work, voluntary activity can be either paid or unpaid - it is the freedom to participate that largely defines voluntary work from other types of work.

As community organisations have realised the need to improve their efficiency and effectiveness, the role of the volunteer has been more narrowly defined through increased formalisation and management control (Morris, 1999; Thornton, 2008). This may include the introduction of role descriptions, formal review and appraisal 
systems, and even disciplinary procedures for volunteers (Bussell \& Forbes, 2002).

With specific reference to the NZ community sector, the Ministry of Social

Development (MSD) found that "the introduction of contracting and the rising concern about standards of service delivery have lead to a growing professionalism in counselling, advocacy, care and protection services" (2001; p. 103). This shift towards increased professionalism and resulting professional development activities that need to be offered by community organisations present some critical issues related to increased investment in training programs, administration, information technology and other support systems (MSD, 2001).

Community organisations have needed to cope with many changes in the general economic and social environments such as changes in employee expectations, an increased need to be socially responsible and fast paced economic changes (Leslie, Lindsay, Mullings \& Salkeld, 1999; Gittell, Ortega-Bustamante \& Steffy, 2000; Brown, 2001). Community organisations are generally not as well equipped as private sector organisations in responding to these changes, as they lack financial and staffing resources.

However, in responding to social changes, community organisations have been shown to be more adept at assisting members of NZ communities that seek a better quality of life and/or a better work/life balance, as they have an objective of improving the quality of their target groups' lives and they also provide a worthwhile means for people to participate in the community (Leslie et al., 1999; Robinson, 2001). It has also been argued that community organisations have developed additional competencies that enable them to adapt to external forces, including:

- An ability to adapt, but stay the same by holding onto their history

- Thinking big and acting small by having a national name and also being rooted in local operations

- Managing multiple stakeholders with disparate interests through active participation in the voluntary organisation

- Making organisational and social values matter by reinforcing the notion that it is not only what gets done that matters, but also how it gets done (Leslie et al., 1999; p. 17). 
Within the third sector SMEs are increasingly important as they are growing in terms of both numbers and their contribution to overall economic growth (Lewis et al., 2007). Indeed, "New Zealand is predominantly a nation of small businesses" (MED, 2007; p. 7).

\subsubsection{NZ SMEs}

The NZ Ministry of Economic Development (MED) defines SMEs as enterprises employing nineteen or fewer employees (MED, 2009). Within this definition, small enterprises are defined as those employing up to five employees and medium enterprises as those employing from six to 19 employees. Previous to this, Yoong \& Huff (2000) conducted a study to explore the adoption of e-commerce by SMEs in NZ. They used the definition offered by Statistics New Zealand, which states that small enterprises are those employing up to 19 staff and medium enterprises employ 20 to 99 staff (p. 2). In congruence with Yoong \& Huff this study has adopted the definition of SMEs given by Statistics New Zealand, as those organisations employing up to 99 staff. This reflects the nature of many community organisations in NZ, particularly those that offer social services, as well as aligning with the definition given in many international studies of IT adoption and diffusion.

As recently reported by MED (2009), within NZ SMEs:

- Contribute significantly to the output of the economy (40\%)

- Make up the predominant organisational form (97\%)

- Constitute nearly all enterprises in the Health and Community Services industry $(92 \%)$

- Account for a significant proportion of total employment (30\%)

It is possible to draw some similarities between SMEs and the head or central offices of community organisations. Of most relevance to this research are the particular factors that impact technology adoption, diffusion and use, specifically:

- The number of staff employed

- The mobile nature of many employees

- The financial restrictions faced

- The lack of technical staff employed 


\section{Number of staff employed \& their mobile nature}

As established above, SMEs employ few staff (up to 99 staff), which is congruent with the average number of staff within the two organisations that participated in this study. The small numbers of employees and the subsequent influence of a single person (for example, an owner/manager) is part of the nature of a SME that makes their general operations, and particularly technology adoption and diffusion, different from that of a large business (Chambers \& Parker, 2000; Al-Qirim, 2007). Many SMEs are reported to lack the traditional hierarchy of structure and systems that larger organisations possess (Swartz \& Boaden, 1997), which has recently been described as a 'weak structure' (Al-Qirim, 2007). SMEs instead may employ a more mobile workforce, and need to support 'any time any place' collaboration and also provide 'universal access' to the services/applications offered by a technology (Munkvold, 2002; MED, 2009).

The NZ Government has recognised the importance of IT adoption by SMEs and Government policy places an increasing emphasis on the 'knowledge economy' and ICT as key drivers for sustainable growth (Locke, 2004). Similarly, the Australian Government has recognised the importance of IT adoption by SMEs, partially due to "their physical (and economic) distance from both metropolitan and international markets" (p. 1). In NZ, the MED have various documents available online that offer SMEs information that highlights the importance of technology adoption to support and improve business practices (see, for example: www.med.govt.nz/irdev/ind_dev/smes2009).

\section{Financial restrictions}

Financial restrictions are another area of similarity between SMEs and community organisations. As for community organisations, a lack of investment capital and/or other financial resources is a barrier for SMEs in terms of general governance and operations, growth, and particularly to those organisations wishing to adopt new ICT (Chau \& Turner, 2002; Lewis et al., 2007; Thornton, 2010). Many SMEs are "resource poor", which has been identified as affecting management processes that subsequently affects the adoption of ICT (Swartz \& Boaden, 1997; MED, 2009). 
Within NZ, recent research has shown that there are many avenues through which SMEs access financial (and other) assistance in order to improve business performance (Lewis et al., 2007). At times, government has been a direct financial contributor to SMEs attempting to improve their work practices and processes by adopting more advanced technologies (Chau \& Turner, 2002).

Many SMEs often state that they cannot afford to upgrade elements of their ICT infrastructure, but as ICTs become increasingly important the question: "Can you afford not to use such technologies?" has been posed (Ariss, Raghunathan \& Kunnathar, 2000). Government programs aimed at improving education and awareness of the benefits offered by technology are a prominent external factor pressuring SMEs to adopt additional technologies (Chau \& Turner, 2002). However, some simple technologies and many more advanced systems are too costly for smaller firms even when the need is clear (Igbaria, Zinnatelli, Cragg \& Cavaye, 1997). For example, Munkvold (2002) found that many organisations stated that the costs involved in installing some meeting support technologies and purchasing a company licence were too high and they instead preferred to use the technology through consulting services provided by academic institutions. Specific to the NZ context, the escalation of costs during a technology adoption project was highlighted as a factor that influenced SMEs decision to adopt new technologies (Yoong \& Huff, 2000).

Several additional financial factors are reported to affect the decision to upgrade technologies: cash flow strength, the availability of financing and government programs specifically designed to support technology adoption (Ariss, et al., 2000; Lewis et al., 2007). These three financial factors may also be faced by voluntary organisations. Despite the many financial costs faced by SMEs adopting new technologies, it is important to note that the use of the Internet is seen by many small businesses as a low cost communication medium (Chambers \& Parker, 2000; MED, 2009). 


\section{Lack of IT staff}

The lack of financial resources described above is linked to another important technology adoption and diffusion issue facing SMEs and community organisations - the lack of information technology staff employed. Compared with larger firms, many smaller firms usually cannot afford to employ internal staff with specialised computer expertise (Igbaria et al., 1997), or they cannot afford to provide the necessary training that existing staff require in order to work on a technology adoption project (Yoong \& Huff, 2000). This may result in a lack of IS human resources, and the necessary IS skills and IS management skills (Ramsey \& McCole, 2005; Cragg, 2008). From this, a 'barrier to understanding' may exist whereby there is a misunderstanding of the consequences of the proposed new technology, or managers may feel that employees are not capable of using or being trained to use the new technology (Ariss et al., 2000). However, Chau \& Turner (2002) found that the level of information technology skills and experience amongst the SMEs that they studied was varied. For example, all had access to the skill sets required by drawing on basic information technology skills acquired by necessity in the past, through family and friends or by undertaking training to further develop their own skills. Training was identified as an important factor of information systems adoption, implementation and use within SMEs (Swartz \& Boaden, 1997), although a lack of resources makes it impossible for many SMEs to establish internal information centres or information systems departments through which training is commonly coordinated (Igbaria et al., 1997).

Many researchers have concluded that a SME might need to seek assistance from an outside technical expert to ensure success of a technology adoption or development project (for example, Swartz \& Boaden, 1997; Cragg, 2008).

\subsubsection{Networked organisations}

Many traditionally structured organisations lacked the flexibility, agility and adaptability necessary to respond to, or keep up with, the quickening pace of business, which has led to the emergence of new organisational forms (Snow, Miles 
\& Coleman, 1992; Garcia, 1993; Franke \& Hickman, 1999; Sandhoff, 1999). In 2001, Chang \& Johnson listed some additional environmental factors attributed to the development of new organisational forms that remain relevant to community organisations today, including the need to:

- Reduce personnel costs

- Keep the number of permanent staff low

- Reduce uncertainty

- Pool knowledge and information

- Create knowledge and information

- Affiliate with credible organisations

While many community organisations do not compete in the traditional, commercial sense, they do often compete for public or private funding and resources (Lewis et al., 2007). Leslie et al. (1999) describe this situation as being in the "market for donations" (p. 16). As competition for resources intensifies, organisations may see it as advantageous to explore alternative organisational processes and forms that may enable them to better compete (Putnam, 1993; Gittell et al., 2000). As Jansen, Steenbakkers and Jagers (1999) argued, "collaboration has become increasingly important for being able to react to the demands of the environment" (p. 55). As such pressures increase with the current economic downturn, community organisations may take on a 'network' form.

Snow et al. (1992) described the social and more prominent economic changes that lead to the decrease in effectiveness of traditional large, hierarchical organisations and the emergence of the new 'business equation' of networked organisations. These authors described a number of social and economic influencers that are pertinent to today's business environment. Social factors include changes in employee expectations, and an increased need for organisations to be socially responsible. Economic turbulence in the form of increasing international competitive pressures results in a high rate of change, or 'dynamism' in the global commercial environment (Snow et al., 1992; Franke \& Hickman, 1999; Peck \& Juttner, 2000). Changes to the hierarchical structures that support traditional organisational forms have also contributed to the emergence of the networked organisation (Townsend, DeMarie \& Hendrickson, 1998; Peck \& Juttner, 2000). 
Snow et al. (1992) discussed three types of network structures: Internal Network, Stable Network, and Dynamic Network, each of which is suited to a specific competitive environment:

- Internal Network: promotes internal efficiencies and innovation through entrepreneurial activities in order to improve organisational performance.

- Stable Network: as for Internal Network, and practices partial outsourcing.

- Dynamic Network: involves extensive outsourcing and is suited to today's international business environment where there exists a high rate of change.

In alignment with the description of the dynamic network, Franke \& Hickman (1999) described another form of network organisation, the 'virtual web' that was created to "promote a new type of organisation that reflects the economical, technological, and social changes in a global environment" (p. 131). This web was described as a group of cooperating organisations that develop relationships through which they pursue collective goals and utilise the resources held by the organisations involved in the network (Snow et al., 1992; Franke \& Hickman, 1999; Haythornthwaite, 2001).

Similarly, an interfirm collaborative linkage can also be described as a voluntary arrangement between independent organisations to share resources, with two distinct benefits:

- Resource sharing: allowing firms to combine knowledge, skills and physical assets

- Knowledge spillovers: serve as information conduits through which news of technology breakthroughs, new insights into problems and failed approaches travels between firms (Ahuja, 2000; p. 427).

A networked organisation has been found to be unrestrained by geography, time and traditional organisational boundaries and thus also called a 'virtual organisation' (Franke\& Hickman, 1999; Jansen et al., 1999;Kimble, Li \& Barlow, 2000). Sandhoff (1999) considered virtual organisations to be networks that operate on principles of self-organisation. These networks are an expansion of traditional organisations and replaced the existing inefficient organisational structures: "networking initiatives are thus a formal recognition and an extension of the way we have always worked in organisations" (Smith \& McKeen, 2003a; p. 3).Kimble et al. (2000) offer another 
more descriptive definition of the virtual organisation: "the inter-organisational arrangement where a group of independent organisations work towards a common goal usually, but not exclusively, using telecommunications and information systems" (p. 3). Also relevant to this discussion are the characteristics of a virtual organisation offered by Jagers Jansen \& Steenbakkers (1998):

- Boundary crossing

- Complementary core competencies/the pooling of resources

- Sharing of knowledge

- Geographical dispersion

- Changing participants

- Participant equality

- Electronic communication (p. 69).

Networks often have immediate, tactical goals ('focussed networks') such as to solve a business problem; improve inter-functional cooperation in a process; collaborate on a project (Smith \& McKeen, 2003a). The business impact, or value, can include stimulating action at local level, motivating people to do things differently, speeding up work, and legitimising cross-boundary conversation (Smith \& McKeen, 2003a).

Many community organisations and SMEs can be described as types of networked organisations, whether for-profit or not-for-profit. The two organisations used in this study possess some of the key characteristics of a networked organisation, specifically:

- Geographical dispersion

- Sharing of information

- Changing participants

- The pooling of resources

- Electronic communication

Each of these characteristics as reflected within not-for-profit community organisations is now briefly discussed. 


\section{Geographical dispersion}

As regional communities are dispersed across a particular country so too are the community organisations that provide them services (Denison, Hardy, Johanson, Stillman \& Schauder, 2002). The individual organisations that make up a voluntary networked organisation participate actively in community development initiatives within their region, often seeking financial and informational resources from within the network (Gittell et al., 2000).

\section{Sharing of information}

Denison et al. (2002) identifies information sharing and knowledge sharing as key network-related activities of voluntary organisations. Providing information and communications channels for staff and other organisational members is fast becoming a key concern of voluntary organisations as they attempt to offer improved services and more effectively engage in community development initiatives (Gittell et al., 2000; Brown, 2001; Denison et al., 2002).

\section{Changing participants}

The very nature of much of the work to be undertaken by many organisations ensures that the organisational participants, whether staff or clients are constantly changing (Leslie et al., 1999; Gittell et al., 2000). Regardless of this constant state of flux, participants of such organisations display a high level of commitment to and ownership of organisational goals and values (Brown, 2001).

\section{The pooling of resources}

Not-for-profit organisations have, for many years, seen the benefit of pooling the limited physical, informational and financial resources of their member organisations in order to continue participating within regional communities (Putnam, 1993; Leslie et al., 1999; Gittell et al., 2000). The pooling of resources is generally seen as a joint, collective (shared) activity, rather than a gift from one person (or group) to another (Robinson, 2001). Contributions may include time, expertise, money or other 
resources and the benefits that accrue from the activity are generally shared. The same arguments can be applied to SMEs

\section{Electronic communication}

As NZ organisations have traditionally been geographically dispersed, with some individual organisations providing services in remote regions, they have relied on electronic communications channels to support and enhance business processes in order to meet organisations goals, and also to communicate to their client base (Denison et al., 2002; MED, 2009). With limited financial resources, some organisations may operate successfully with the support of simple communication technologies such as telephone and facsimile, while others have invested in developing Internet-based communications solutions to support their business processes and informational needs (Denison et al., 2002). The ICTs typically adopted by community organisations are further discussed in section 2.3 .

\subsubsection{Section summary}

Community organisations have an increasingly important role within the 'third' sector of NZ. The discussion above has shown that, while there are unique elements of community organisations and their operating environment, such organisations also have strong similarities with both SMEs, such as:

- Number of staff employed

- Mobile nature of many employees

- Financial restrictions faced

- A lack of technical staff employed

- Geographical dispersion

- Sharing of information

- Changing participants

- The pooling of resources

- Electronic communication

The networked nature of many community organisations and SMEs results in a reliance on ICTs to support their processes and practices. This reliance, coupled with 
factors such as those listed above presents significant challenges to the leaders of community organisations, especially in the introduction of innovations with organisation-wide impacts.

\subsection{Technology Support for NZ Community Organisations and SMEs}

This section includes a discussion of the provision of technology within community organisations and SMEs. As established in section 2.1, there are strong similarities between community organisations and SMEs, and indeed many community organisations can be categorised as 'human services' SMEs. Therefore, this section provides an overview of technology support for community organisations by drawing on literature on for-profit and not-for-profit community organisations as well as SMEs.

The adoption and ongoing use of technology by NZ SMEs has been found to have a positive relationship with organisational growth (Locke, 2004) and an increasingly important impact on business effectiveness (Al-Qirim, 2007; Cragg, Tagliavini \& Mills, 2007). Indeed, Cragg (2008) stated that IS competencies are a pre-requisite for building organisational capabilities in small firms. The adoption, diffusion and use of technology are distinct yet related aspects of technology provision, and therefore each is outlined separately below. As the focus of this study was on the actual use of computing resources, additional information and supporting literature can be found in later chapters of this thesis.

\subsubsection{Technology adoption}

Factors influencing the adoption of technology by community organisations can be broken into three main categories: innovation characteristics, adopter characteristics (such as their education) and behavioural characteristics (such as the existence and actions of change agents) (Rogers, 1995). This covers the three prominent areas of technology, people and the context in which these two interact. Two additional 
categories of environment characteristics and task characteristics (such as task structure and autonomy) were later added (Chambers \& Parker, 2000). These authors also divided adopter characteristics into organisational characteristics (such as company size and funding/time resources) and individual characteristics of adoption decision makers (such as their age, level of education and experience). Combined, these authors provide a definitive list of categories of factors that affect the adoption of technology by SMEs.

Researchers have found that it is inadequate to introduce a new technology without also paying attention to the effect of the new technology on existing work processes and practices (Chau \& Turner, 2000; Munkvold, 2002; Al-Qirim, 2007). In particular, Cragg et al. (2007) conducted a study to explore the operational alignment of IT with business processes within NZ SMEs, and found that each organisation typically had five processes with insufficient support.

Ramsey \& McCole (2005) identified many factors that may impact positively or negatively on the IT adoption process, including awareness and understanding, technical compatibility and complexity. As Munkvold states, "there is a complex change process often associated with the implementation of a new technology (Munkvold, 2002; p. 363). When discussing technology enabled business transformation, Chau \& Turner (2000) state that the benefits to an organisation of improved IT are achieved when investment in IT services are matched by corresponding changes to organisational processes. Similarly, Munkvold (2002) found that the introduction of new technologies also raised debate about the adequacy of existing work practices (Munkvold, 2002; p. 358). Part of such a change process may involve the preparation of guidelines for the effective use of technology related to accessibility, ownership and use of technology services or the information managed by the technology (Munkvold, 2002; Ramsey \& McCole, 2005).

In a case of adoption and diffusion of an electronic meeting system some critical success factors were found, including: organisational commitment, the need for an executive sponsor, training, facilitation support, dedicated meeting facilities, 
cost/benefit analysis and meeting managerial expectations (Munkvold, 2002; p. 359). Many of these factors involve dedicated 'support roles' such as facilitators, product champions and change agents. Chau \& Turner (2000) list leadership as a 'managerial factor' under their discussion of organisational factors affecting the adoption of technology by SMEs, and specific to the NZ context, Cragg (2008) found that several managerial factors had a significant influence on IS success and concluded that "technical capability is a significant factor that differentiated IT leaders from laggards" (p. 32). Further to this, Cragg, Mills, and Suraweera (2010) argued that top management support is but one aspect of IT management that makes a critical difference to IT adoption. Other IT management practices Cragg et al. (2010) found to have influence include:

- IT planning: - determining what is to be achieved, setting goals, and identifying appropriate action steps. Planning centres on determining goals and the means to achieve them.

- IT organising: allocating and arranging human and material resources in appropriate combinations to implement plans. Organizing turns plans into action potential by defining tasks, assigning personnel, and supporting them with resources.

- IT controlling: monitoring performance, comparing results to goals, and taking corrective action. Controlling is a process of gathering and interpreting performance feedback as a basis for constructive action and change.

- IT leading: guiding the work efforts of other people in directions appropriate to action plans. Leading involves building commitments and encouraging work efforts that support goal attainment.

Also relevant to the NZ context, Al-Qirim (2007) found that the individual factors of innovativeness and prior IT knowledge influenced the adoption of internet-based technologies within SMEs. The leadership qualities of managers are central to the adoption and continued utilisation of a new technology: "the introduction of innovation requires time and personnel to integrate, train and maintain new systems within an organisation" (Chau \& Turner, 2002; p. 213). Within many smaller organisations the owner/manager also took an active role in the development, implementation, utilisation and maintenance of the new technology applications when possible (Chau \& Turner, 2002; Al-Qirim, 2007). 
Despite the increased success of ICT adoption within SMEs (MED, 2009), there are also many reasons why ICT is not adopted and/or used, including: lack of management enthusiasm, innovativeness and entrepreneurship, external pressure and support, perceived lack of suitability for their organisation, its complexity and cost, need for immediate returns, resistance to change, survival in the short term, and the perceived security risks of technology (Ramsey \& McCole, 2005; Al-Qirim, 2007; Cragg, 2008).

\subsubsection{Technology diffusion}

Once a new technology has been implemented, managers and other executives must ensure that organisation wide diffusion of the technology is achieved. This has been termed the process of building a 'critical mass' of users within an organisation (Munkvold, 2002). Email has been identified as an example of a technology that is often successful regarding diffusion and user adoption, due to its intuitive nature and the clear analogy to 'traditional mail', or the traditional work practice that it is enhancing (Munkvold, 2002; Smith \& McKeen, 2003a). Other technologies, for example Instant Messaging (IM) and social networking sites are also classed as easily diffused due to the support that they offer the informal communication tasks of employees (Munkvold, 2002).

Offering a complimentary view, Cragg (2008) matched managerial practices to IS competencies and found that six practices differentiated IT leaders from IT laggards, three reflected senior management involvement and three reflected technological capability. These six practices were matched to ten competencies that make a difference to IS success in small firms, as presented in Table 1 below. 
Key managerial practices

Role of IT within the business

Senior management commitment to IT.

Managers seek new uses for IT.

Customisation of new IT systems

IT specialist

IT development skills.

\section{Related IS competencies}

Business strategy - ensure that business strategy formulation identifies the most advantageous uses of information, systems and technology.

IS strategy alignment - ensure that IS development plans are integrated with organisational and functional strategic plans.

Benefits planning - explicitly identify and plan to realise the benefits from IS investments.

Managing change - make the business and organisational changes required to maximise the benefits without detrimental impact on stakeholders.

Technology innovation - incorporate the potential of new and emerging technologies in long term business development.

Systems and process innovation - carry out relevant R\&D into how IS/IT can be used to create new ways of conducting business and new products and/or services.

Technology analysis - understand technology trends and make appropriate recommendations for organisational acquisition of technology and associated resources.

Applications development - develop/acquire and implement information, systems and technology solutions that satisfy business needs.

IS/IT staff development - recruit, train and deploy appropriate staff and ensure technical, business and personal skills mee the needs of the organisation.

Apply technology - deploy new/changed technology in the most cost effective mode to deliver application benefits.

Table 1: Managerial Practices and IS Competencies (Adapted from Cragg, 2008)

Despite the successful adoption and diffusion of technologies into many community organisations, various potential diffusion problems should be highlighted. Social interaction between employees and employees and external persons (for example, clients, and partners) can be diminished as electronic communication supersedes the 
use of face-to-face interactions. Fink \& Disterer (2006) found that although staff may understand the potential for technology to support their communications, "its use may be ameliorated by strong considerations for the human element" (p. 619).

In one reported case, Munkvold (2002) found that organisational members experienced an over-reliance on the use of a new workflow management system for communication, which led to a reduction in human interaction. Within the same case, employees also felt increased work pressure due to the possibility of surveillance by management through the system. The diffusion of technology can also be hampered by other factors such as insufficient user training in the use and potential of the introduced technology, a lack of explicit routines for effective technology use, and a lack of incentives for information sharing (Quadus \& Xu, 2002; Ramsey \& McCole, 2005).

Difficulties such as these can be overcome through strategies such as:

- Establishing universal access to the services enabled by the technology (Munkvold, 2002)

- Building a 'critical mass' of experienced technology users that can assist other users (Quadus \& Xu, 2002; Munkvold, 2002)

- Creating new incentive systems to stimulate employees' technology use (Munkvold, 2002)

- Carefully assessing task factors to determine whether the factors are conducive to technology use (Quadus \& Xu, 2002)

- Initially restricting the use of the collaboration technology with new routines (Munkvold (2002).

\subsubsection{Technology use}

After technology has been adopted and begins to diffuse through the organisation, the actual use of the technology should be examined. DeLone \& McLean (2010) include systems use as a key measure of IS success, and state that researchers must "consider the nature, extent, quality and appropriateness of the system use" (p. 16). 
There are three categories of technology use developed by Karsten (1999) and discussed by Munkvold (2002):

- Exploratory, conservative or cautious use: technology was only used to automate existing routines, limited in scale, no major applications were developed

- Planned and expanding use: implemented initially restrictive applications, but organisations had plans for expanding these

- Extensive and engaged use: the user took an active role in integrating technology applications into their work. The nature and amount of collaboration was also changed (p. 362).

Extending this, Chau \& Turner (2002) found that the use of a newly adopted technology could be classed as strategic, operational or experimental. Experimental use was evident at early stages of technology adoption and diffusion; operational use covered medium-term day to day tasks; later stages of diffusion involved a more strategic and expanding approach to technology diffusion. One critical challenge was stated as providing the right "balance between management directives and user experimentation for fostering creative incorporation and application of the technology in the user's day" (Munkvold, 2002; p. 363).

Briggs, Nunamaker \& Tobey (2001) propose that as people begin to use technology, they perceive value (both cost and benefit) along a number of dimensions, among them:

- Affective: changes in positive or negative feelings

- Economic: changes in cash, assets, marketability, etc

- Physical: changes in health and comfort

- Political: changes in the balance of power

- Social: changes in relationships

When people contemplate the use of technology and evaluate it along such dimensions as these they enter a 'transition' period, that begins with expressed interest and ends when a 'community' or 'critical mass' of users has become selfsustaining. (Briggs, Adkins, Mittleman\& Kruse, 1999). Some indicators that users have become self-sustaining are: they use the technology without intervention from a change agent; new staff are trained and socialised to use the technology as a matter 
of course; organisational practices and/or processes are adapted to incorporate technology use; and, if the technology breaks, the users find a way to get it fixed, rather than abandoning it (Briggs et al., 1999; Ramsey \& McCole, 2005). DeLone \& McLean also add another dimension here, that the technology is used for the intended purposes. This is similar to the idea of the 'faithful appropriation of a technology and/or its particular features, as discussed in Chapter 7. As organisational members increasingly use a technology, they may express their needs of the technology more clearly. This may stimulate further improvements and changes to the particular technology, or perhaps even changes to organisational processes will be needed to ensure that the technology becomes an integral part of peoples' work processes (Cecez-Kecmanovic, 2002).

It has been found that technology is needed to support and enable SMEs. In NZ the degree of ICT penetration within SMEs is high (MED, 2007) which suggests that ICT (including online resources) are not merely an enabler, but a vital component of these organisations. While it has been recognised that research into technology use by not-for-profit organisations is still relatively new (Denison et al., 2002), the technologies used within distributed organisations has been well documented. One factor contributing to and enabling the operation of the distributed organisation is the continuing advancement and adoption of information and communication technologies (ICT) (Sandhoff, 1999; Lanam, 2001). ICT was viewed as a driving force towards new organisational forms: "the emergence of powerful information and communication technologies have further facilitated the move towards networks and 'virtual organisations' are increasingly a feature of the business landscape" (Tidd et al., 1997; p. 324). The role of technology within distributed SMEs today is more prominent, ICTs have become a precondition or a prerequisite for organisational success, as predicted (Jagers et al., 1998; Sandhoff, 1999).

The technology utilised by traditionally structured organisations worked to bring employees together in a central office. In contrast, recent technologies enable employees to work remotely, although still for a particular organisation. This has resulted in work diffusing rather than concentrating, forcing organisations to rethink 
the structure of the organisation and the interactions that take place as part of day-today work (Snow et al., 1992; Jagers et al., 1998; Franke, 2001). Other authors concur, finding that recent advancements in ICTs have forced organisations to re-evaluate their structure and work processes and placed more attention on the virtual organisation and mobile work (Townsend et al., 1998; Ellison, 1999). These statements demonstrate the importance placed on the role of technology within the distributed organisation. Furthering this idea, Kimble et al. (2000) described the physical and electronic environments as co-existing and complimentary. This shows that technology can be viewed as a different infrastructure for the way people relate to one another within distributed organisations. The person(s) responsible for coordinating a distributed organisation will rely on the "latest information and communication technology (Internet/intranet) to facilitate the collaboration between individuals and organisations across space, time and organisational boundaries" (Franke \& Hickman, 1999; p. 122).

The use of computing resources within community organisations ranges from a large scale infrastructure that includes all organisations in a cooperating network, and offers Internet based training, education and content development to relatively small scale technology use within an individual organisation (Denison et al., 2002). Online resources have been used within community organisations for such activities as cultivating donors, highlighting awareness of organisational activities through web sites and email lists, conducting virtual meetings and more general community interactivity (Allen, 2003). As suggested by Fink and Disterer (2006) it is preferable to integrate the use of ICTs with more traditional communication mediums, such as mail and phone.

ICTs are required to not only enable communications and knowledge sharing, but to enable such exchanges to take place in a timely manner to allow continual feedback and interaction (Garcia, 1993). Organisational responsiveness and the effective use of time are seen as essential in today's environment. Kimble et al. (2000) describe time from the resource viewpoint, arguing that time can be traversed by the use of ICTs and more specifically by online resources to connect network members from 
around the world, around the clock, potentially extending productive time to 24 hours a day. Regardless of how broad ICT use is, communication technologies assist a community organisation to increase the "speed and spread of community engagement" (Denison et al., 2002; p. 6).

In his discussion of the Virtual Web Organisation Franke (2001) extended previous research through an elaboration of the role of technology in networked (distributed) organisations. He described four types of information systems that cross organisational boundaries and enable the timely and extensive exchange of information and knowledge. The four main ICT types are communications systems, administration systems, project management systems and supply chain management systems as presented in Table 2. The importance of technology for new organisational forms is simply put: "the possibilities of the virtual organisation grow ever larger as a direct result of ICT” (Jagers et al., 1998; p. 73).

\section{System Category}

\section{Communication:}

email, video conferencing, electronic notice boards

\section{Administration:}

databases, marketing systems, personnel systems, legal software

Project management: groupware integrating electronic messaging with screen sharing, scheduling, group writing etc.

\section{Supply chain management:} resource planning software, work in progress management, inventory databases

\section{Description}

Used for the exchange of information between members of the networked organisation. Communications systems facilitate the integration of the network and external suppliers and customers.

Important for the management of the networked organisation. Holds information about organisational structure, policies and processes, employees, products and services and network members.

Necessary for activities such as product development and research. Groupware supports team leadership, facilitates group processes and the sharing of ideas and enables parallel task execution.

Coordinates the flow of information and materials from the customer to the supplier. Necessary to support the various supply chain configurations possible with the dynamic nature of the networked organisation.

Table 2: The Main Categories of Information Systems (Franke, 2001; p. 54-55). 
Specific to the NZ context, organisations are seen as successful in applying technology in interesting and new application areas (Symonds et al., 2003). In NZ, the most extensively used technologies have been reported as 'Internet' (73\%), 'intranet' (70.4\%) and 'online information sources' (58.1\%) (Symonds et al., 2003; p. 1157). Document repositories/ document management technologies were also featured on this listing. Despite enthusiasm for new online resources, lengthy transition times have been found to inhibit the widespread acceptance and use of a new technology:

- The technology may evolve faster than it can be assimilated into daily work practices

- Short-term financial constraints may use up resources needed to sustain transition

- Technology champions/experienced users may move on - technology languishes and dies (Briggs et al., 1999; p. 152).

In summary, ICTs and online resources are viewed as a necessity for any successful community organisation and can enhance information exchange and knowledge sharing, improve the speed and spread of interactions, and support the overall organisational structure adopted. The most effective type(s) of technologies for each community organisation will depend on the particular structural type and services offered by that organisation. Although there is a relatively low degree of use of online technologies in some not-for-profit community organisations, broader technology use as discussed above contributes to the development of online resources by raising awareness of the many-to-many potentiality of online technologies.

\subsubsection{Section summary}

As illustrated in the above discussion, there are many factors to contemplate when considering the adoption, diffusion and use of technology within community organisations. Technology adoption, diffusion and use by community organisations can be problematic and undoubtedly requires strong leadership from management and/ or other top-level executives to guide the many activities that must be undertaken. The adoption, diffusion and use of technology is examined in this 
research project to support the professional development activities of a group of practitioners within community organisations, as next discussed.

\subsection{Professional Development}

Today's business environment is one characterised by rapid and ongoing change, and in response practitioners are challenged with continual learning in order to acquire the necessary knowledge, skills and abilities of their particular profession. Such professional development can be defined as the "systematic, ongoing, self directed learning" of organisational practitioners (Jones \& Robinson, 1997; p. 198).

Professional development is viewed differently in different organisations, some including it as a component of an overall training and development strategy, while other organisations have developed specific professional development policy statements or objectives linked specifically to business performance goals (Jones \& Robinson, 1997). Any ways that professional development can be linked to strategic areas of the business or business planning could be a valuable justification for devoting resources to training and development (Jones \& Robinson, 1997). However organisations choose to view professional development, some support must be provided that could come in the form of making time available for the necessary activities or making available the financial resources needed.

Recent research by Coetzer and Perry (2008) highlighted the differences in approaches to professional development between large and small firms within NZ. Particularly important was the focus by large organisations on formal training, while small organisations focussed more on informal training and learning processes. As such, much previous research into learning within SMEs was criticised as it treated the presence or absence of training as a measure of learning. Coetzer and Perry (2008) distilled their findings of employee learning in small NZ firms into four broad themes:

1. Factors in the external business environment (including learning resources) 
2. Factors in the work environment (such as employee practices and resource constraints)

3. Learning potential of the job itself (i.e. the kinds of tasks that an individual engages in can be a primary source of learning)

4. Learning orientations of employees (ranging from unmotivated to proactive)

Taken together, these themes provide a holistic picture of the types of elements that need to be taken into consideration when developing, delivering, and/ or managing professional development programmes within SMEs.

One factor that variously spans the external business environment and the internal work environment is the more recent proliferation of online professional development, also termed distance professional development. Lock (2006) describes this as the 'new image' of professional development, within which geographically separated practitioners are provided with ongoing development opportunities that are based on their needs, as opposed to organisational objectives. Online professional development currently has many definitions and takes many different forms, from single-episode training modules, to the use of learning management systems, to fully immersive learning environments. One common practice is that of blended professional development, whereby online technologies are applied to complement face-to-face professional development events (Green \& Cifuentes, 2008). An online component may be used before and/or after an event to prepare or follow up with participants, and provide an environment within which peer interaction can be established and/ or maintained outside of the face-to-face event (Bernard, de Rubalcava \& St-Pierre, 2000; Green \& Cifuentes, 2008). In a study of groups of school librarians, Green and Cifuentes (2008) found that those who participated in online peer interactions following a face-to-face workshop had a different, and more positive, experience than those who did not participate online. This shows that organisations and their employees can still benefit from online support, without a full online professional development programme being necessary.

From an organisational viewpoint, by engaging in professional development activities, a practitioner is expected to demonstrate an ability to perform to acceptable standards, having regard to the changes and challenges which accompany 
all organisational activity (Jones \& Robinson, 1997). The outcomes of professional development activities must also be assessed, and how they can be used to enhance personal performance in a work role. This indicates that there needs to be a strong connection between learning and change - application oriented learning generates incremental changes over time as new knowledge and skills are put to use (Eisen, 2001). There also needs to be the application of learning, i.e. using the new skills or knowledge in work situations, and the internalisation of learning, i.e. adding a new dimension to professionals' personae, for professional development to be successful (Eisen, 2001).

In their study of professional development in university settings, Weaver, Robbie and Borland (2008) synthesised their findings into three key elements argued to be necessary for successful, institution-wide implementation of professional development:

- Full support of the institution/ senior management is obtained, at the policy level

- Professional development is delivered by staff with recognised expertise and credibility in the subject area

- The professional development programme is wide-ranging, (including flexibility and transferability in timing of professional development, type of professional development, and resources etc) (p. 767).

Many organisations focus on external development activities and overlook the internal learning environment where coaching and mentoring may take place. The traditional concept of professional development was the hierarchical structure of the mentor-protégé relationship, within which the person being mentored had a development relationship with some one in the organisation who is more senior or more experienced in a particular area of interest to them (Holbeche, 1996; Stead, 2005). This fosters a power imbalance as well as a one way flow of information from the mentor to the novice. Mentoring can help employees to refine their organisational role, prepares them for advancement and provides a 'social sphere' in which they receive role modelling, experiential learning, counselling and friendship (Siegel, 2000; Stead, 2005). Many organisations still adopt the mentor-protégé professional development form and in some situations it is appropriate, for example mentoring senior level executives as part of a leadership development programme (Stead, 2005). 


\subsubsection{Peer consulting}

For many professionals (even if at the early stages of their career) the traditional form of professional development, the mentor-protégé relationship, often fails to tap into the knowledge and expertise that they have already developed (Eisen, 2001). Therefore, peer mentoring, which I have termed peer consulting for the purposes of this research, offers professionals a powerful alternative for personal and professional growth (Holbeche, 1996; Siegel, 2000).

In the organisational situation where groups of practitioners face similar problems, approaches to professional development that incorporate the concepts of communities of practice and knowledge sharing are especially appropriate. Peer consulting is one such approach where "two, three or more individuals agree to have a development relationship with one another which may involve occasional or regular meetings, phone calls, exchanges of information and specific forms of support which go beyond networking" (Holbeche, 1996; p. 25). Essentially, it is the sharing of people's experience through action and reflection in the context of actual practice (Eisen, 2001). Peer mentoring relationships can be established across organisational functions or divisions, and even go beyond the organisation as a means of addressing both organisational needs for teamwork and greater collaboration and individual needs for support (Holbeche, 1996). In contrast to a mentoring arrangement, the peer consulting development relationship is one of equality between members of a peer group. Within peer-to-peer consulting, peer dynamic is the term given to the distinctive nature of the peer relationship. This can be characterised by the following defining qualities:

- Trust (feeling safe)

- Non-evaluative feedback

- Non-hierarchical status of partners

- Voluntary participation and partner selection

- Duration and intensity of partnership (leading to closeness)

- Mutuality (common goals and reciprocal learning)

- Authenticity (openness, honesty) (Eisen, 2001; p. 37). 
As indicated by these relationship qualities, instead of learning from each other, peers learn with each other in a reciprocal relationship. Similar to the concept of peer consulting, Eisen (2001) described peer learning partnerships as "voluntary, reciprocal helping relationships between individuals of comparable status, who share a common or closely related learning/ development objective" (p. 31). Such learning is said to be especially good for adult learners, as they can bring their life and work experiences to a learning activity or problem situation. Tapping into one another's ideas allows them to leverage their existing storehouse of expertise (Holbeche, 1996; Eisen, 2001). One way to exchange experiences is through storytelling, in which peers interact reciprocally to share and construct practice-related knowledge and strategies (Orr, 1990). This is becoming an increasingly powerful way to exchange tacit or 'soft' knowledge and for practitioners to arrive at their own solutions to a problem (Hildreth et al., 2000).

In addition to storytelling, professional development in peer groups may involve offering advice, making suggestions, critiquing ideas, offering alternatives, or simply listening (Holbeche, 1996). Skilled listening and clarifying behaviours by peers are critical to allow people to reach the level of understanding that leads to problem resolution. Peers could also use the consulting relationship to help one another gain access to support and information, which helps them be more effective in their own work situations (Holbeche, 1996). Within the peer relationship, great value is placed on formative, reciprocal feedback that emphasises development rather than evaluation (Eisen, 2001). This type of feedback is enabled by trusting and open relationships where the sharing of knowledge occurs between peers unimpeded.

The activities undertaken in a peer consulting arrangement can have the some times unintended effect of improving a person's confidence and breaking down the geographical and/ or professional isolation that may have existed. Sustained peer relationships over an extended period of time can help professionals to develop a continuing sense of competence and identity (Siegel, 2000). However, the peer consulting relationship is based on open sharing of knowledge, and as such peers may need to break down any insecurities or suspicions that are present before peer 
mentoring can take place. Eisen (2001) suggested that it may be appropriate to offer training before embarking on a peer learning situation - training in being receptive to feedback, knowing when to act on it, and even invite it may facilitate adult learning and professional growth.

A fundamental element of professional development and peer consulting is the sharing of knowledge. Knowledge sharing among professional practitioners is next discussed.

\subsubsection{Knowledge sharing}

In today's environment, effective communications and knowledge sharing among organisational members is a vital ingredient for the success of any organisation, and especially resource-poor community organisations, and can provide an organisation with some form of advantage over others (Townsend et al, 1998; Quadus \& Xu, 2002). This argument is extended by the assertion that knowledge is increasingly seen as central to organisational success and an asset that needs to be managed (Kimble et al., 2000; p. 2). However, many organisations still retain a functional orientation that keeps knowledge of one function in isolation of the knowledge of other functions, thus inhibiting wider professional development.

Various groups of practitioners are seen as playing a critical role in creating, maintaining, and sharing knowledge within today's organisations. Theories of knowledge typically encompass the two broad categories of explicit and tacit knowledge. Nonaka, Toyama and Konno (2000) state that explicit knowledge can be formally articulated and written down, whereas tacit knowledge is highly personal and harder to formalise. Tacit knowledge includes individual insights, intuitions and 'hunches' that are disseminated through social acts of sharing between peers (Nonaka et al., 2000). In organisational settings, knowledge is continually created through the SECI conversion process that encompasses four stages of: 
1. Socialisation, sharing tacit knowledge among individuals

2. Externalisation, articulating tacit knowledge into explicit concepts

3. Combination, combining different entities of explicit knowledge

4. Internalisation, embodying explicit knowledge into tacit knowledge (Nonaka, von Krogh \& Voelpel, 2006).

Through these four processes, "by interacting and sharing tacit and explicit knowledge with others, the individual enhances the capacity to define a situation or problem, and apply his or her knowledge so as to act and specifically solve the problem" (Nonaka, von Krogh \& Voelpel, 2006; p. 1182).

Such exchanges may take place within learning communities (see section 2.4 below). In organisational settings, communities of practice ( $\mathrm{CoPs})$ are viewed by some as the 'glue' of knowledge management, or knowledge in action: "CoPs form the foundation of knowledge management because it is through them that knowledge gets both created and turned into action" (Smith \& McKeen, 2003b; p. 14). CoPs can contribute to the sharing of knowledge within organisations by understanding which knowledge domains are essential to the organisations success; determining who is in the best position to manage the knowledge in that domain; creating new knowledge and scanning the environment for new knowledge sharing technologies and/ or methods (Wenger, 2002) . $^{3}$

Organisational networks generally have official endorsement from management that gives organisational members permission to interact and share with others outside their immediate department or functional group. The term network can be taken to represent interpersonal, non-hierarchical connections between individuals, business units or organisations along which knowledge (i.e., information plus interpretation) flows (Smith \& McKeen, 2003a). As evident in the CoP discussion below, social interaction networks may form naturally within an organisation, or may be initiated by management or other individuals within an organisation (Gongla \& Rizzuto, 2001; Smith \& McKeen, 2003a). More specific to the discussion of knowledge sharing

\footnotetext{
${ }^{3}$ Communities of practice are discussed further in section 2.4.
} 
among peers for professional development, Gongla and Rizzuto (2001) describe knowledge networks as "institutionalised, informal networks of professionals managing domains of knowledge" (p. 843). Such professionals could be both within and outside of a particular organisation.

Smith \& McKeen (2003a) discuss two identifiable networking goals related to knowledge sharing: to facilitate knowledge search and to facilitate knowledge transfer. Knowledge search has to do with connecting people or groups in a variety of non-routine ways, which can be an important source of new ideas and help organisations extend and develop their capabilities. Networks are used to make sure that all relevant information is available and that it can be quickly found, thus the network needs to be broad in scope and connect diverse sources of information. Knowledge search can be facilitated by such actions as publishing a 'yellow pages' of 'who knows what', or identifying key individuals who are well connected and who are tasked with providing links. Knowledge transfer, as described by Smith \& McKeen (2003a), can be hindered or facilitated by certain individuals, and the type of knowledge involved can affect the ease with which it moves. More complex knowledge needs stronger and closer ties between a few individuals, while less rich media and weaker relationships are acceptable for transferring less complex knowledge (for example, social news).

Knowledge sharing is a complex and dynamic process, within which change management, organisational learning, organisational processes and information technology need to be skilfully blended to ensure knowledge sharing is successful (Symonds et al., 2003). Knowledge managers can add value to an organisation if they can assist with bringing together these various areas to help the organisation to filter, analyse and distribute the information that networks generate more effectively, and enable the creation of knowledge (Smith \& McKeen, 2003a). If a professional development network includes members from outside a particular organisation, a knowledge manager may be tasked with assisting the group in clarifying who owns information when exchanges take place, and what will happen to any new knowledge that is generated as a result of the interactions of professional development activities. 


\subsubsection{Section summary}

Professional development takes many forms, as appropriate within different organisational settings. Traditional forms of professional development are still relevant and practiced within many organisations, such as workplace learning through a mentor-protégé relationship. However, these traditional professional development arrangements are being extended, such as the recent management development technique of peer mentoring, as well as being complemented by other professional development practices.

The groups of peers that meet, physically or virtually through ICTs, can be viewed as a type of community; the terms learning communities, knowledge communities, communities of inquiry, communities of interest and communities of practice have all been used to describe different forms of professional development community. The term and form that is most relevant to this particular study is the learning community, as explored in the next section.

\subsection{Learning Communities}

Recently, a new paradigm of professional development that is based on a community philosophy has emerged (Lock, 2006). Learning communities are underpinned by the same core elements of a Community of practice ( $\mathrm{CoP}$ ), namely domain, community and practice. These elements are interwoven with some key factors of professional development:

- A collection of individuals (online or offline) undertaking professional development activities would form a community

- They would be professionals interested in learning from each other through discussing and solving problems of practice

- Their community would be formed around a particular domain of knowledge.

- Also, within the distributed environment of a community organisation an online component to a professional development programme could assist practitioners in forming the community of practice within which professional development activities take place.

The CoP concept is explored in more detail throughout the remainder of this section. 


\subsubsection{The nature of communities of practice}

CoPs have been described as "groups of people who share a concern, a set of problems, or a passion about a topic, and who deepen their understanding and knowledge of this area by interacting on an ongoing basis" (Wenger, McDermott \& Snyder, 2002; p. 4). Within CoPs members typically discuss ideas, explore them together and get feedback from their peers. Gongla \& Rizzuto focus more on the organisational context in their definition, of "a collection of individuals bound by informal relationships that share similar work roles and a common context" (2001; p. 842). Some researchers, such as Saint-Onge and Wallace (2003), challenge each organisation to develop a definition for CoPs that is meaningful to their particular situation.

CoPs are not new, and have been operating within organisations for many years. However, their informal nature often means that they rarely come into explicit focus and attract attention. The practitioners from a specific professional area within an organisation who need to collaborate to solve practice-related problems in order to learn from each other can be termed a CoP. To determine the actual make up of a $\mathrm{CoP}$, it is useful to consider some aspects of 'community':

- They vary enormously in their size and duration

- They include some sense of belonging to a group

- While communities include some people, they inevitably serve to exclude others

- As well, some interests of members are denied, just as some are affirmed (Bruce \& Easley, 2000; p. 243).

The interaction of the elements of domain, community and practice can be combined into a process of learning through active social participation (Moore \& Barab, 2002; Conrad, 2005). Within this process, rich sets of relationships and responsibilities around learning enable active questioning as a way for members of a CoP to critically reflect on real life problems (Piggot-Irvine, 1996; Wenger et al., 2002; Lock, 2006). Summarising these points, Smith \& McKeen, (2003b) view a CoP as "a group of people with a common interest who work together informally in a responsible, independent fashion to promote learning, solve problems, or develop 
new ideas" (p. 3). In summary, CoPs are viewed as social mechanisms within which people assist each other in a somewhat stable environment.

In keeping with the view of a $\mathrm{CoP}$ as a social mechanism, the collaborative learning activities that take place within communities are often described as social processes (Bernard et al., 2000). Creating a strong and shared sense of community, also termed a 'social climate' (Saint-Onge \& Wallace, 2003), is increasingly important as the complexity of learning activities within a $\mathrm{CoP}$ increases, and as over time isolated learning events become continued professional development (Conrad, 2005).

In addition to the three interrelated elements of domain, community and practice, five characteristics of a CoP have been highlighted:

1. Because a CoP must develop over time, it has a history of learning

2. It has an enterprise (focused activity or endeavour), something which forms around a value-adding something-we're-all-doing, but it does not have an agenda of action items as a team would

3. Learning is a key element of this enterprise and as a result, CoPs develop their own ways of dealing with their context

4. They are responsible only to themselves and self-policing. Leaders tend to emerge on an issue by issue basis. In addition, because relationships within a $\mathrm{CoP}$ are ongoing and indeterminate, they tend to be characterised by mutual trust

5. CoPs are concerned about content rather than form and as a result, they are often not identifiable or designable units (Smith \& McKeen, 2003b; p. 3).

As indicated above, a $\mathrm{CoP}$ is different from an organisational team. A team typically comes together to complete a pre-specified task, so they are together because of the interdependency of specific tasks on which they are working (Lesser \& Prusak, 1999; Wenger, 2002). In contrast, and of most relevance to the discussion of CoPs for continuing professional development, in a $\mathrm{CoP}$ people are brought together through the interdependency of their knowledge. More recently, some researchers have argued that CoPs are a vital component of an organisations knowledge management programme, delivering value in terms of knowledge creation, capture, storage and dissemination (see, for example the work of Hildreth and Kimble, 2000 \& 2005; Desouza \& Awazu, 2006). 
Within an organisational context, CoPs emerge and develop in a variety of ways. ${ }^{4}$ Organisational knowledge flow is said to occur naturally, whereby the exchange of ideas between individuals who have experience and skill related to the same area of work takes place. This interaction with others on work-related topics has been found to lead naturally to the formation of communities of practice (Gongla \& Rizzuto, 2001; Akkerman, Petter \& de Laat, 2008).

There are two common approaches of identifying potential CoPs. The first is to find informal or social groups already operating around the organisation's core competencies and help them come together as communities. This is termed 'matchmaking', whereby organisations locate and link people or groups of a particular domain in order to initialise a CoP (Gongla \& Rizzuto, 2001). The second approach to identifying a $\mathrm{CoP}$ is to begin with a business model and ask "what types of knowledge communities will enhance this model?" (Smith \& McKeen, 2003b; p. 9). A strategic element that helps to link the $\mathrm{CoP}$ to organisational performance can assist in answering this question.

Once a CoP has been initiated, attention usually falls on the nature of community activity, or the degree of participation that occurs. There are different, legitimate types of participation in a CoP. Smith and McKeen (2003b) describe the existence of a core group of participators and peripheral forms of participation as well. They divide participation into three stages, which are necessary in becoming a full member of a CoP:

- Finding the community and 'lurking' to see what is going on

- Learning how to get around and participate in the community

- Knowing how and where to break the rules and to innovate (Smith \& McKeen, 2003b; p. 4)

\footnotetext{
4 Appendix A provides detail of three prominent CoP development models that provide an interesting view of the stages a CoP moves through and some of the social, process and technology related issues that affect community initiation, activity, growth and sustainability.
} 
Signs of full membership in a CoP include shared tacit conventions, understandings, and assumptions and a common worldview (Gongla \& Rizzuto, 2001; Smith \& McKeen, 2003b).

Another perspective on activity at the early stages of a $\mathrm{CoP}$ is offered by Akkerman et al. (2008), who found that there are three dimensions to community activity, namely:

1. Meaningful activity: identify and define the domain, develop shared needs and clear objectives

2. Shared activity: activity that is relevant and focused, and draws on any shared history among participants

3. Coordinative activity: provides shared direction for activity.

Whichever view of community activity is taken, it is important to note that within the membership of a CoP there are no pointed distinctions between experts and novices, instead there is the recognition that each person has experiences that are valuable and worth sharing with the group. This aligns with the concept of peer consulting (as described in section 2.3) which emphasises equal relationships among peers and the value of their individual experiences and knowledge.

Alongside community activity, the facilitation or support provided to community members is also important for community development. It is argued that all CoPs benefit from some form of 'cultivation', and require different types of support (Smith \& McKeen, 2003b). There are three general areas in which an organisation can provide such support:

- Management: create and environment through their actions, reinforce norms, help to set expectations, provide them with needed resources, recognise their importance in the organisation (Gongla \& Rizzuto, 2001; Smith \& McKeen, 2003b)

- Technical infrastructure: Technology is useful in helping a community to connect, and also expands the reach of a CoP (Wenger, 2002; Smith \& McKeen, 2003b).

- Culture: there is a need to develop an organisational culture that supports communities (Saint-Onge \& Wallace, 2003; Bernard et al., 2000) 
To integrate CoPs effectively into organisations, managers need to find out how to collaborate and how to learn, as an organisation, from collaboration (Saint-Onge \& Wallace, 2003). There is no identifiable point or stage at which a CoP has developed completely and offered all the value that it can. Instead, CoP members will continually share their insights, questions and frustrations in order to help each other develop a richer and more fruitful understanding of an area of practice (Bruce \& Easley, 2000). In summary, it is the exchanges of ideas, best practice and new knowledge that CoP members value the most, and it is the positive impact that these exchanges have on an individual's practice that gives value to the wider organisation.

\subsubsection{Technology use within communities of practice}

As described within this literature review, community organisations are often geographically and temporally distributed, and in NZ many are spread across the length of the country. Therefore, it is vital that the professional development activities of groups of peers (communities of practice/learning) are supported by available technologies.

CoPs existed long before modern ICTs were available. Today ICT is viewed as an enabler and enhancer of the activities of CoPs and a facilitator of knowledge sharing, although it is not going to make the critical difference to many CoP activities (Wenger, 1998; Cecez-Kecmanovic, 2002; Desouza \& Awazu, 2006). More specific to the learning communities of this study, Lock stated:

"Designing an online learning environment that fosters the development of a learning community is not about adding technology onto current professional development practices. Rather, it is about designing, building, and supporting a structure and a process that are purposeful and fluid in nature and meeting the personal, ongoing professional development needs of teachers." (2006; p. 663)

Community development and knowledge sharing are viewed as social and cognitive processes, thus a technological capability alone is unable to dissolve many 
organisational and knowledge sharing barriers and motivate people to share (Symonds et al., 2003). These authors argue that as ICT electronically transmits data or information, but not knowledge; also, they are facilitators of information transmission and distribution, not a substitute for human interaction and communication. Once ICTs are in place, successful communication, collaboration and knowledge sharing depends on members of a CoP using the technologies, rather than on the organisation having the technological capability to share knowledge. However, people who live in remote areas and wish to participate in a CoP may be concerned about the complexity that their geographical isolation adds to communication: "how does one remain connected with people hundreds of miles away, where they may not even be a connecting road?" (Bruce \& Easley, 2000; p. 247).

Communication through electronic networks may be established, allowing employees involved in CoPs and collaborative work to participate from remote locations (Bruce \& Easley, 2000). CoPs need tools that enable members to maintain contact with each other, and also allow them to identify potential new members. Personal web pages, directories of expertise and knowledge maps can assist here, enabling members of a $\mathrm{CoP}$ to locate others with similar interests and experiences and develop relationships that lead to knowledge sharing and build social capital (Lesser \& Prusak, 1999). Synchronous collaborative tools such as online chat rooms and videoconferencing can also help community members to establish and maintain connections (Lock, 2006). Electronic networks are also used to share practice related ideas and discoveries, tips for equipment use and, often, frustrations with technology between a diverse, but cooperating community (Bruce \& Easley, 2000).

Despite the necessity of knowledge sharing within CoPs, and the technology needed to support them, some challenges and risks exist. Conflicts with formal and informal ways of working and changes to organisational processes may not be easy to conceptualise, especially if the needed technology or the understanding of $\mathrm{CoP}$ principles is under-developed (Smith \& McKeen, 2003a). Training is essential for the effective use of technology, and part of developing an adequate organisational 
technology capability. Organisations with low technology use have been found to have low skills and experience to implement IT to support knowledge sharing (Symonds et al., 2003). The speed of change in information technology and the uncertainty of knowledge requirements have also been identified by knowledge managers as issues (Smith \& McKeen, 2003a; Symonds et al., 2003). Although presented with such challenges, pressures on modern business including technological advancements are encouraging organisations to look for ways to leverage social networks and take advantage of the propensity of people to connect with others to improve business performance.

In summary, knowledge sharing technological solutions are not the 'be all and end all' of successful knowledge sharing, but they are seen as the foundation that facilitates human interaction and communication. The importance of technology to support knowledge sharing has been recognised: "the foundation of knowledge sharing in NZ business depends on the IT systems implemented and there is still a lot to find out about IT knowledge sharing systems" (Symonds et al., 2003; p. 1161). Although current networking technologies can bridge geographic and time boundaries and support CoPs, it has been found that many fall short of providing practitioners with exposure to the actual experiences required for learning (Moore \& Barab, 2002).

\subsubsection{Online communities of practice}

Hildreth, Kimble \& Wright (2000) posed the question "can CoPs operate in the international distributed environment?" They argued that some aspects of a CoP should translate from the more traditional, co-located environment to the distributed or virtual (online) environment relatively easily. One such community aspect is finding a common purpose or shared interest. $\mathrm{CoP}$ members who are doing a similar job will already have a shared interest, domain language and knowledge. When assessing whether a $\mathrm{CoP}$ can function in a distributed environment, it is important to identify the reasons, if any, that a CoP needs to be co-located. This question can be framed "what is the reason for situatedness?" (Hildreth et al., 2000; Lee, 2004). If it 
is because they need to share resources such as a document, then it should translate to the distributed environment relatively easily. If the learning is situated because $\mathrm{CoP}$ members need face-to-face contact for seeing how a job is done then distribution will be more difficult, but perhaps still achievable.

Another consideration when initiating an online $\mathrm{CoP}$, is the social, temporal and physical isolation, or 'periphery' that exists in the virtual environment instead of just social periphery in the co-located environment. The facilitation of participation has therefore been identified as one of the most difficult areas in the transition to the online, distributed environment (Hildreth et al., 2000). As in the co-located environment, participation is central to the development and sustainability of an online community and to the creation of relationships that help develop the sense of trust and identity that defines the community.

When describing two case studies, Hildreth et al (2000) highlighted the importance of a 'physically co-located element' to a CoP. They found that electronic communication was at the highest level after a face-to-face meeting, and then communication gradually declined until being rejuvenated or motivated by the next face-to-face meeting. In addition, the authors found that the development of relationships in the face-to-face environment helped with issues of identity. CoP members got to know each other better more quickly then if they were developing the relationship via 'e-media'. Having good personal relationships with other CoP members meant that they had confidence in what they were receiving from the other members, whether it was information, the solution to a problem or simply an opinion.

As discussed in previous sections, when undertaking a $\mathrm{CoP}$ and knowledge sharing initiative, organisations need to know what technology is able to provide, and have some knowledge sharing technological capability to be able to exploit it (Symonds et al., 2003). In addition to the ICT previously discussed, examples of technologies appropriate for knowledge sharing within a $\mathrm{CoP}$ are data warehousing, groupware, the Internet and intranets (Cecez-Kecmanovic, 2002; Symonds et al., 2003). However: 


\begin{abstract}
"Given the expense of video conferencing and groupware technologies relative to online technologies and the availability of online technologies and the high standard of telecommunications infrastructure within New Zealand it seems that more and more companies will be adopting online technologies to facilitate knowledge sharing" (Symonds et al., 2003; p. 1160).
\end{abstract}

Therefore, of most relevance to this discussion of online CoPs are the Internet and intranets. The Internet is a useful source of information and intranets often provide organisations with a knowledge sharing framework, as they allow for sharing of information at the document level rather than the record level provided by other, traditional systems (Symonds et al., 2003). The importance of sharing at the document level was highlighted in the research paper of Hildreth et al (2000). They found that shared documents were often a 'collaboration catalyst' in that they were the catalyst for members to apply their domain and soft, or tacit knowledge. Collaborations that may take place in the co-located environment can be reproduced electronically in an online CoP via shared resources such as documents. In an organisational context such at that of this research, intranets are seen as well suited to support knowledge sharing within CoPs as they:

- Provide compression of time and space

- Offer flexibility to exchange information

- Support information transfer and organisational networking independent of contact between users (Symonds et al., 2003; p. 1152).

The support of information transfer without contact between users is as close to knowledge sharing that a technological solution can come without human intervention (Cecez-Kecmanovic, 2002; Symonds et al., 2003). Some common applications of online knowledge sharing systems are: organising and sharing or transferring of internal benchmarks and best practices; constructing corporate knowledge directories, such as corporate yellow pages, people information archive, etc; creating knowledge networks and knowledge maps (Quaddus \& Xu, 2002; p. 76).

Different approaches to online collaboration and learning may vary in terms of the particular technologies that are used and the level of interactivity that they offer, but 
their common aim is to deliver collaboration and learning opportunities to members of a community at disparate locations (Stokes, 2001; Lee, 2004). Benefits of online collaboration and learning approaches include the ability to connect the people who are the best sources of knowledge with those that need the knowledge and are at different locations, decreased travel costs and the enabling of asynchronous communications (Leser \& Prusak, 1999; Stokes, 2001).

An example of a technology that has been used by a community of practice to support professional development activities is the Inquiry Learning Forum (ILF) (Moore \&Barab, 2002). The focus of ILF was not on the use of the technology itself (the Internet, in this case), but on promoting inquiry-based methodologies for teachers. The ILF community "is a resource designed to facilitate continuous professional development and enable sharing among community members at all stages of skill development from master teachers to teachers in training." (Moore \& Barab, 2002; p. 45). For more experienced teachers ILFs goal is to "provide a vehicle for discussing practice and advancing community as well as individual understanding. Through observation, discussion, and reflection, each participant can find his or her own path to continued professional growth and development" (Moore \& Barab, 2002; p. 45).

Hildreth, et al. (2000) offer a second example of an online CoP. They described a case study of a management team of IT support in the research arm of a major international company based in the USA. Four members co-located in the UK, a group of five members in the USA and one member in Japan made up the CoP that was studied. The group managed to successfully function as a $\mathrm{CoP}$ in the distributed environment. Members met on a twice-yearly basis, and in between these meetings they maintained communication via 'e-media' such as email, voice mail, video link and Microsoft NetMeeting. Within this online CoP the speed or immediacy of interactions was of the greatest importance when specifying their media preference for a particular task. 
While an online CoP has some challenges to consider that are not so pertinent for colocated CoP members, the above discussion and examples given show that online CoPs are a feasible option and offer members some important benefits. Whether face-to-face, online or a combination of both, communities of practice are an effective forum for professional development activities to take place.

\subsubsection{Section summary}

While CoPs may take many differing forms and be comprised of various characteristics, they are essentially organised around the three distinct and common elements of Community, Domain and Practice. Communities of practice allow groups of people form relationships through which they engage in discussions based around issues that are important to their area of practice. Organisations have realised that employees hold a vast amount of soft, or tacit knowledge that, when shared, can greatly improve aspects of work practices and other aspects of work situations. Communities of practice offer a way of effectively transferring this knowledge between practitioners while also building social capital and many organisations are realising the benefits of this.

However, as the discussion in Section 2.1 of the literature review showed, many organisations operating in today's business environment are geographically dispersed, which acts to isolate many practitioners from the benefits of being involved in CoPs. Various ICTs including peer-to-peer computing and the Internet and intranets are ways to enable and support distributed communities, leading to the formation of online communities of practice. When formed, CoPs offer a forum in which professional development or more specifically peer consulting, can take place. Supported by technology, such communities can reach practitioners that were previously professionally isolated and enable them to give and receive peer consulting. 


\subsection{Literature Review Summary}

The literature review has introduced the nature of the community organisation and highlighted some of the similarities they share with NZ SMEs, including:

- A relatively small workforce

- Providing a large proportion of NZs social services

- Generally resource poor

- Geographic dispersion, operating in cities and rurally

- Increasing reliance on ICT

As the number and contribution of NZ SMEs continues to grow, it is important that researchers investigate this sector further and bring to light aspects of SME best practice, organisational experiences, strategies and more general advancements into a cumulative body of knowledge (Lewis, Massey \& Harris, 2007).

One such aspect of SME practice that this study aims to explore is the nature of ICT adoption, diffusion and use by community organisations. Although use of both online and other ICTs by SMEs has been steadily increasing, there exists little knowledge of the extent to which effective application of online ICTs are a precondition to regular and ongoing professional development activities.

Within the organisational context, professional development activities are often undertaken by groups of peers. For the purposes of this research, such groups are termed learning communities, the concept of which draws heavily from the well established and researched principles of communities of practice (CoPs). Section 2.4 discussed the three distinct yet interconnected CoP elements of domain, community and practice and how these come together within learning communities to provide an environment conducive to active and meaningful peer reflection and practice-related learning. Of most relevance to community organisations that are geographically dispersed is the online CoP, supported by various ICTs. Such communities can reach practitioners that were previously isolated (socially and/or professionally) and enable them to engage in peer consulting. 
Through the course of merging these different literatures, a gap in current knowledge has been highlighted regarding the way in which community organisations apply and use online ICTs to enable groups of dispersed practitioners to engage in professional development activities. It is to this body of knowledge that the results of this study can contribute most, as described throughout the remainder of the thesis. 


\section{Methodology}

\subsection{Chapter Overview}

This chapter describes the research method that was used for the collection, organisation and analysis of research data associated with this study. The opening sections discuss the rationale for using interpretive, qualitative research and applying the Action Research method. Following this, section 3.4 provides a more detailed explanation of the action research cycle and how it was applied in this study, introduces the research organisations and reviews the tensions between pursuing both practical and research goals simultaneously. The following sections introduce the researcher role at action research stages, more specifically the particular data collection and analysis techniques applied.

\subsection{Research Paradigm: interpretivism}

Information systems research is typically guided by an overarching paradigm which Orlikowski \& Baroudi (1991), following Chua (1986), separate into positivist, critical and interpretive. Positivist studies on information systems have been widely accepted and published, and the results of interpretive studies are increasingly published and discussed among the research community.

Within the positivist philosophy, the researcher acts as an independent outsider, to observe the 'laboratory' or research context without influencing the object under study. The researcher that ascribes to the positivist philosophy typically assumes that phenomena under study is identifiable, tangible and divisible into component parts and thus can be observed objectively and rigorously, and that good research is 
legitimated with reference to repeatability, reductionism, and refutability (Jonsson, 1991; Braa \& Vidgen, 1999). Commonly, an intervention made within a study following the positivist philosophy must be controlled so only the experimental variable changes, and the prevailing elements (such as organisational context) are held constant in order to provide replicability and predictive power (Orlikowski \& Baroudi, 1991; Braa \& Vidgen, 1999). Typically, information systems (IS) research can be classified as positivist if there is evidence of formal propositions, quantifiable measures of variables, hypothesis testing and the drawing of inferences about a phenomenon from a representative sample to a stated population (Jonsson, 1991; Klein \& Myers, 1999).

The critical approach to research is distinguished by its evaluative dimension, whereby the researcher attempts to critically evaluate and elaborate and/or transform the social reality under investigation. The goal of most critical research is to expose inequalities and conflicts in a social situation, and the researcher role is "to bring to consciousness the restrictive conditions of the status quo" (Orlikowski \& Baroudi, 1991; p. 21). Researchers who ascribe to the critical philosophy believe that if individuals are made aware of restrictive conditions in their environment in terms of position, tradition, resources, power etc, they can act to influence structures and ultimately improve possibilities to realise their potential (Jonsson, 1991).

In contrast to the positivist philosophy, the interpretive philosophy considers the methods of natural science to be inappropriate where human beings are concerned, mainly because different people (including researchers) will interpret a situation in different ways (Orlikowski \& Baroudi, 1991). This holds especially true when the interpretivist researcher attempts to understand meanings that research participants use to make sense of their lives and social structures within which they operate (Lau, 1997). As Jonsson (1991) stated, "the core of the interpretive undertaking is to understand action on the actors terms" (p. 377). Within the IS discipline, the goal of interpretive methods is to help us to understand the context of an information system, and the process through which the information system influences and is influenced by the context (Myers, 1997). Within interpretivist studies, this understanding comes 
through the knowledge of the reality of a situation that is gained only through social constructions such as language, consciousness, shared meanings, documents, tools and other artefacts (Klein \& Myers, 1999). In brief, "typically, positivism is concerned with reducing the area of investigation in order to be able to make reliable predictions and explanations, while interpretivism is concerned with making a reading of a situation in order to gain understanding" (Braa \& Vidgen, 1999; p. 2).

A key tenet of the interpretive perspective is that organisational reality is assumed to be produced and reproduced by human beings through their action and interaction (Jonsson, 1991). As such, relationships between people, organisations and technology are not fixed but constantly changing, as are any observable organisational patterns. As a consequence, interpretive research seeks to understand a moving target from the perspective of the participants (Klein \& Myers, 1999). Exposing different logics, or multiple ways of understanding the world, that arise from these different organisational circumstances and forming concepts and meanings based on them is another hallmark of interpretive research (Eisenhart, 1998).

Table 3 provides an overview of some important characteristics of the positivist and interpretive paradigms, argued to have more or less influence on the quality of different types of IS research (Weber, 2004).

This current study has followed the interpretive paradigm, as intervening directly into organisational contexts in order to effect some change and explore the process of applying online ICTs to support professional development activities necessitated the researcher closely observing, collaborating with and undertaking sense-making activities with participants. Further, as the 'reality' of the situations under study were largely made up of observing the actions of participants and examining reports of their perceptions and experiences centred on their own interpretations of research events and activities, the positivist and critical approaches were not appropriate. 


\section{Metatheoretical \\ Assumptions About}

Ontology

Epistemology

Research Object

Method

Theory of Truth

Validity

\section{Positivism}

Person (researcher) and Reality are separate.

Objective reality exists beyond the human mind.

Research object has inherent qualities that exist independently of the researcher.

Statistics, content analysis.

Correspondence theory of truth: one-to-one mapping between research statements and reality.

Certainty: data truly measures reality.

\section{Interpertivism}

Person (researcher) and reality are inseparable (life-word).

Knowledge of the world is intentionally constituted through a person's lived experience.

Research object is interpreted in light of meaning structure of person's (researcher's) lived experience.

Hermeneutics, phenomenology etc.

Truth as intentional fulfilment: interpretations of research object match lived experience of object.

Defensible knowledge claims.

Table 3: Characteristics of Positivism and Interpretivism (adapted from Weber, 2004; p. iv)

Although the nature of differences between the characteristics of positivism and interpretivism are still under debate among some IS theorists (as summarised by Weber, 2004), they provide a valuable set of elements with which to consider the quality - in terms of evaluation of the results - of a study (discussed further in Chapter 8).

As Trauth (2001) noted, interpretivism is the philosophy that most often influences the choice of qualitative methods, as it has for this research (Trauth, 2001). It is also important to acknowledge that the word 'qualitative' is not a synonym for 'interpretive' and that it is the underlying philosophical assumptions of the researcher that influence whether qualitative or quantitative research is chosen for a particular study (Myers, 1997; Klein \& Myers, 2001). The choice of qualitative research approach is next discussed. 


\subsection{Qualitative Research Methodology}

Research methods are commonly separated into the two categories of quantitative and qualitative (Creswell, 1994). Quantitative research methods have traditionally received a wider application and the results of quantitative studies have been more accepted by the academic community. The debate over recent years regarding the application of quantitative and qualitative research methods has seen increasing acceptance of qualitative methods and their outcomes, meaning that qualitative researchers do not need to engage in lengthy justifications of their choice of methods or the credibility and validity of research results (Lau, 1997; Baskerville \& Wood Harper, 1996).

This study has adopted a qualitative approach for a number of reasons. Firstly, qualitative methods are most effective in answering research questions that are descriptive and often the focus of these questions changes and evolves as the research progresses. These are described as 'how' and 'why' questions (Benbasat, Goldstein \& Mead, 1987). The research questions posed in Chapter 1 of this thesis fit within the qualitative paradigm as they indicate a process of investigation and inquiry.

Secondly, qualitative research methods are designed to help researchers to understand and/or interpret a social or human problem and the social and cultural contexts within which they occur (Myers, 1997). In this study the main source of data collected was participants' descriptions of the adoption and use of online resources within their organisation. This data were used to uncover each participant's perceptions and experiences, thus obtaining the views of participants to build a 'picture', as common in qualitative studies. The ontological position is therefore that "the only reality is that constructed by those who are participants in the research situation" (Creswell, 1994; p. 4), including myself. Therefore, the second reason for the selection of qualitative methods is that the study focused on human perceptions and experiences. 
Thirdly, the researcher was actively involved in the process of the adoption, dissemination and use of online ICTs by participants and moved freely around each organisational environment to observe happenings first hand in order to construct an accurate description of participants' experiences. Participants were observed in their 'natural setting' to gain insights into the social and interpersonal interactions that help to form their reality (Benbasat et al., 1987). Therefore, a high level of ongoing interaction was achieved between the researcher and participants, in the organisational context.

Fourthly, qualitative research is usually undertaken over a sustained period of time, in which the relationship between the researcher and the participants is expected to develop (Creswell, 1994). The data collection period of this research took place over a period of approximately two years, resulting in sustained involvement within the participants' environment and the development of strong researcher-participant relationships that facilitated the collection of observation and interview data and meaning-making that occurred at various stages of the study.

In summary, qualitative research was chosen for this study, as it is suitable to the research questions posed, allowed the researcher access to the organisational settings over a sustained period of time and to examine human perceptions and experiences of a particular process from their own perspective. 


\title{
3.3 Action Research
}

\author{
"Action research aims to contribute to the practical \\ concerns of people in an immediate problematic \\ situation and to the goals of social science by joint \\ collaboration within a mutually acceptable ethical \\ framework" (Rapoport, 1970; p. 499). This definition \\ highlights that action research should be grounded in \\ the concerns of practitioners and should be \\ collaborative in nature; that it involves research as well \\ as action; and that it should contribute to research \\ knowledge as well as attempt to resolve particular \\ issues. The use of action research entails some \\ assumptions about scientific knowledge that are not \\ widely institutionalised, and fall within the interpretive \\ philosophy: "by being interpretive, the researcher is \\ engaged in social construction of reality where he or \\ she attempts to understand the social phenomena \\ within a naturalistic setting" (Lau, 1997; p. 40).
}

Action research is a phased approach to research that involves the researcher actively intervening into an organisational problem situation to effect some desirable change. "The organisational laboratory provides researchers with a rich setting for investigating the work, management, and technology issues associated with information systems. Such investigations are not necessarily passive affairs; the researcher can also be involved in making an intervention in the problem situation with the aim of achieving some desirable change" (Braa \& Vidgen; 1999; p. 1).

Action research is a context bound inquiry, as the researcher is attempting to solve real world, practical problems, and the research participants from a particular organisation are the ones who experience and define such problems. There are three primary aims of action research: organisational development, system design and scientific knowledge (Baskerville \& Wood-Harper, 1998). This research was focused on organisational development by improving the working (and potentially social) conditions of the organisational participants through implementing online systems for use in professional development activities. This aligns with the organisational tradition of action research whereby the researcher acts to intervene in an 
organisation to improve its design or development (Lau, 1997). Changes to the peer consulting process combined with technological changes necessitated researcher intervention into the organisational situation. Chisholm \& Elden (1993) support this, arguing that "organisational and system change involve complex social processes that require sustained strategic interventions to bring about change" (p. 293). Interventions can range from a specific intervention, to a series of related tasks, to that of an action-oriented learning or systems methodology.

The ideal domain of the action research method has been revealed in three distinctive aspects of the method:

1. The researcher is actively involved, with expected benefit for both the researcher and the organisation.

2. The knowledge obtained can be immediately applied. There is not the sense of the detached observer, but that of an active participant wishing to utilise any new knowledge based on an explicit, clear conceptual framework

3. The research is a cyclical process linking theory and practice (Baskerville \& Wood-Harper, 1996; p. 239).

The selection of action research for this study centres on three issues. Firstly, the nature of the interaction between the researcher and organisational participants is a key aspect of a research paradigm. As Elden \& Chisholm (1993) note, action research would be impossible without some form of participation: "it requires those who experience or "own" the real world problem to be actively involved with the researcher at least in selecting the problem and sanctioning the search for solutions" (p. 129). The need for interaction may come from the outside researcher's need for insiders' deep understanding of context and culture in a system where research is to be meaningful to system members (Braa \& Vidgen, 1999). The level of interaction in an action research study depends on a variety of conditions, including the researcher's skill and expertise levels, and participation in all action research phases is not uncommon.

Within this study the researcher experienced a moderate level of interaction with research participants, although this varied during different stages of the action 
research cycle. The researcher was not immersed in the organisational situation to become an organisational member, nor acted as a silent and distant observer. The intervention level for this study involved participants undergoing a period of change; from having few professional development opportunities and processes, to utilising online resources for specific peer consulting activities. As mentioned above the researcher did operate within the organisational situation in order to observe the experiences of research participants as they unfolded. There were times where, due to the sensitivity of a particular situation, it was not appropriate for the researcher to intervene or participate in organisational activities, but receive instead a summary of events, decisions and/or issues from a key participant (or participants).

The action researcher assumes that complex social processes can be studied best by introducing changes into these processes and observing the effects of these changes (Baskerville, 1999). The introduction of online ICT into two social services organisations and the observation of ICT use by a community of learners necessitated my active participation and intervention in organisational social systems. As research participants used the online ICTs for professional development activities their needs became clearer, and the researcher actively participated in the change and discovery process by modifying ICTs to better meet users' needs.

Also, application of action research enabled a strong and direct link to exist between the theory generated through this research (the key research results discussed in the following thesis chapters) and the actual practice that was enacted by participants. As Lau stated: "For those who aspire to make a difference in the field of IS, action research provides a unique opportunity to bridge theory with practice, allowing one to solve real-world problems while contributing to the generation of new knowledge" (1997; p. 61).

In summary, action research allowed interaction between the researcher and participants in their natural organisational settings. This interaction involved some degree of participation between the researcher and participants, in order to successfully introduce process and technological changes into the participant 
organisations. This collaborative process ensured a positive relationship between the theory generated during the research and the practice itself. As Meister and Gronski (2007) noted when detailing the benefits of using action research, value lies in the research results being the 'results' (or findings) of the participant organisations and in results being actionable items that were tested, evaluated and refined through ongoing dialogue with research participants.

\subsubsection{Action research in the IS field}

Action research has been identified as an alternative mode of inquiry that has been used in the field of social science since the 1940s as a research strategy that integrates theory and practice through change and reflection (Lau, 1997). When discussing the 'origins' of action research the work of researchers such as Lewin (1951) and Trist (1976) is commonly cited and the later, information systems (IS) specific work of authors such as Susman and Evered (1978) and Checkland (1981) is typically discussed. More recently, Baskerville \& Wood-Harper (1998) detailed the historical development of action research in the IS field. The period from 1990 to the present day is described as the fourth main period of action research development, where diffusion of the method takes place, and the various forms of action research are applied appropriately for various useful research purposes.

The number of action research studies from within the IS discipline has increased over recent years. Conditions have changed to create an environment that stimulates and is receptive to the various applications of classical action research. As the organisational environment has changed, the action research method has changed in response: "action researchers have developed new ways of thinking about research that would solve practical problems and contribute to general scientific theory" (Elden \& Chisholm, 1993; p. 122). Many of the conditions that have changed reflect those discussed in section 2.2 of Chapter 2 that enabled the emergence of the networked organisation. Chisholm \& Elden (1993) specifically discuss the importance of networks, as a type of organisation: "networks provide a flexible means of involving diverse constituents in AR in ways that fit with the goals, context, 
and varying degrees of interest required by the research process in specific settings" (p. 293). Conditions that affect the nature of social and organisational research include:

- Increased interdependence among various types of organisations/ institutions/groups/ key people makes it more difficult to develop neat, clearly bounded research designs or pre-conceived research plans

- A faster pace of change and increased complexity (related to interdependency) cause a more turbulent environment for action research

- An increased expectation for real involvement and participation in developing solutions to problems

- Practitioners have demanded increasing involvement in designing and conducting research (Chisholm \& Elden; 1993; p. 276).

These changing conditions coupled with the complexities of examining the interaction of people and technology in an organisational setting offer challenges for the IS researcher. In response, action research is a method that is increasingly deemed appropriate for exploring and resolving research problems in the IS discipline (Lau, 1997).

'Action research' is often used as an overarching term that encompasses different research approaches, ranging from more formal approaches through to less formalised, more reflective and personal approaches, such as participative action research and more recently also Distance Action Research (DAR) whereby action research is carried out while participants and researchers are geographically (and sometimes temporally) separated. It is necessary for the researcher to distinguish the type of action research used and its historical context from which the purpose, focus, theory and methods of the study are based (Lau, 1997). There are four sub-classes of action research that are commonly identified: action science trying to resolve conflicts between espoused and applied theories; participatory action research emphasising participant collaboration; action learning for group experiential learning and action research focussing on change and reflection. While this study shares some characteristics with such approaches (such as their collaborative nature and group focus) it has followed the classical, five-staged action research methodology as described below. 


\subsection{The Action Research Cycle}

Several key action research practitioners (Elden \& Chisholm, 1993; Baskerville, 1999; Baskerville \& Pries-Heje, 1999) have discussed a simple, two-stage action research process consisting of the diagnostic and therapeutic stages. The diagnostic stage generally involves a collaborative analysis of the social situation by the researcher and the subjects of the research. This includes specifying which actions are to be taken, formulating theories concerning the nature of the research domain. The therapeutic stage is where the collaborative change experiments are undertaken, changes are introduced and the effects are studied. The classical or 'canonical' action research process, originally attributed to Susman and Evered (1978), offers additional structure and was adopted for this study. Action research varies greatly by the degree of openness, or structure, of the research process itself, and the degree to which the specific research steps are specified prior to the research intervention taking place (Chisholm \& Elden, 1993). A relatively closed action research method involves researchers predetermining how the research will be conducted, identifying key aspects of the situation for study, and then carrying out the research (Chisholm \& Elden, 1993). While I specified such aspects of the research process in advance, I also allowed decisions about later research steps to be derived from findings and learning in earlier stages of the study.

Figure 1 illustrates the classical action research process, as followed in this study. This process involves the five interrelated phases of diagnosing, action planning, action taking, evaluating outcomes and specifying learning (Baskerville, 1999; Lau, 1999; McKay \& Marshall, 2001). 


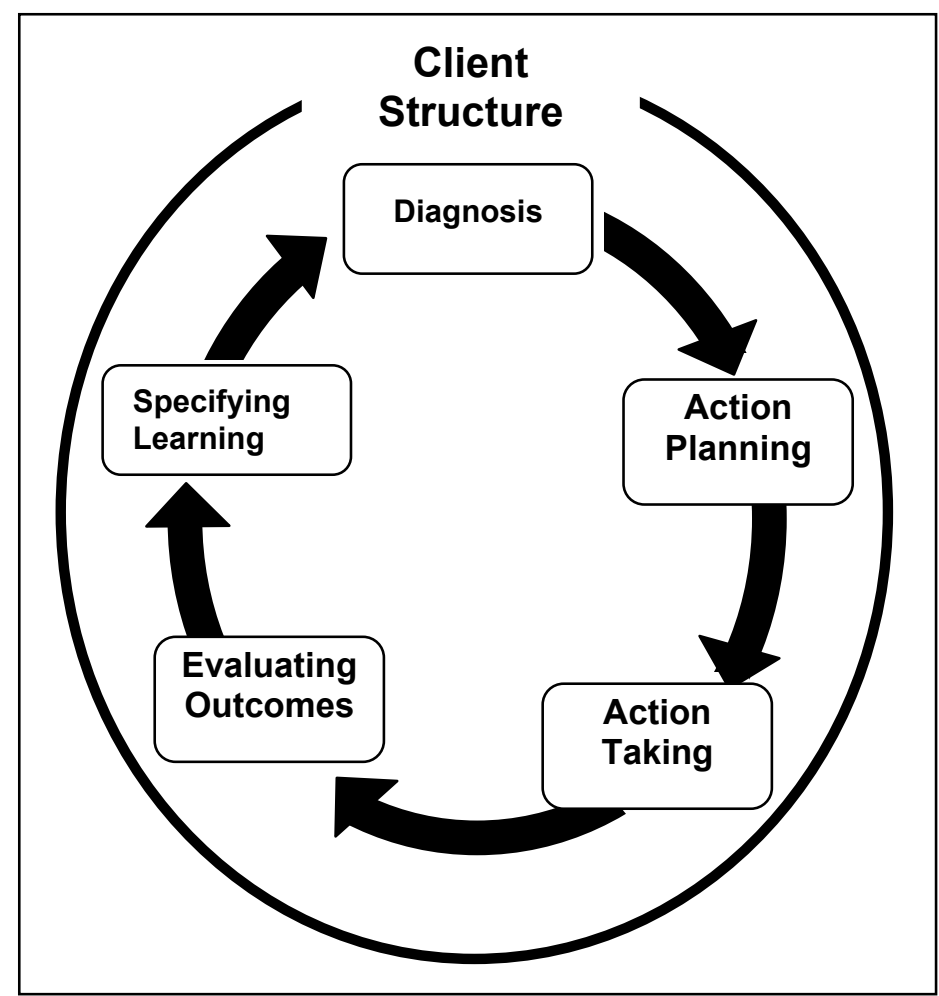

Figure 1: The Action Research Cycle (Baskerville \& Pries-Heje, 1999; p. 4).

\subsubsection{Dual goals of action research}

Action research has been described as an approach that has "a dual goal of benefiting a research client and, at the same time, generating relevant research knowledge" (Kock \& Lau, 2001; p. 2). This has been described as two concurrent cycles of activity, a problem solving interest cycle and a research interest cycle as described by McKay and Marshall $(1999,2007)$ depicted in Figure 2.

With particular regard to the role of intervention, Braa \& Vidgen (1999) state that the goal of action research is "to gain learning and knowledge through making deliberate interventions in order to achieve some desirable change in the organisational setting" (p. 2). Action research provides a unique opportunity to bridge theory with practice, and allows a researcher to solve real-world problems while generating new knowledge. Implicit in the discussion of an action research intervention pursuing contrasting goals is the idea that the information systems action researcher serves 
two different 'masters': the research client (an industry, organisation, department or individual) and the research community as a whole (Kock \& Lau, 2001). The researcher undertakes some form of collaboration with research participants in order to solve the identified problem and effect some long lasting change in the organisation whilst also generating knowledge to communicate to the academic community (Lau, 1999).

With regard to this study, as the researcher I acknowledged the need to be effective within the participant organisations in order to effect change and alleviate their organisational problem and also give due attention to the research activities at all stages of the action research cycle so that the research goal of generating new knowledge could be achieved. With limited resources at my disposal I undertook careful planning of stages of the action research cycle, paying particular attention to time allocated to data collection and analysis activities in addition to change related activities that benefit the participant organisation and individual research participants.

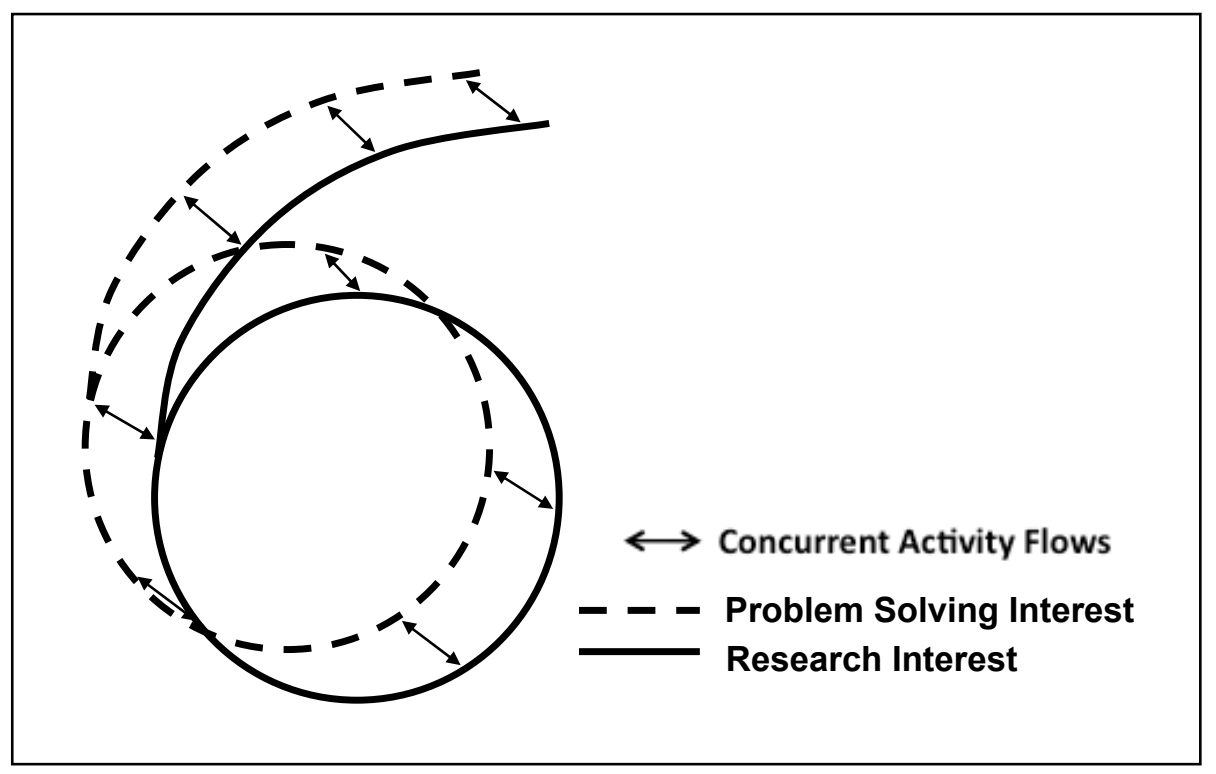

Figure 2: Action Research Viewed as a Dual Cycle Process (McKay \& Marshall, 1999; p. 603).

At different stages of the action research cycle the amount of effort and resources devoted to the problem solving interest cycle versus the research interest cycle changed (see Figure 2). Therefore, I adjusted my activities accordingly to complete both the research and problem solving activities necessary to achieve the objectives of this research. 


\subsubsection{The client structure}

The action research cycle exists within a particular organisational environment, also called the client-system infrastructure (Baskerville \& Pries-Heje, 1999) or the organisational context (McKay \& Marshall, 2007). This is represented in Figure 2 as the Client Structure that encompasses the five action research stages. The client structure has also been variously described as the social climate in which action research takes place (Olesen \& Myers, 1999). Too little importance is often placed on understanding the context, including the formal and informal social environment, in which an action researcher operates (Olesen \& Myers, 1999; Avison et al., 2001). Indeed, Olesen \& Myers (1999) reflected on a particular action research project in which the social and organisational context within which information technology was implemented had a profound effect on the way in which the technology was used. They also concluded that project failure was due to institutional forces, which inhibited significant changes in the work habits of research participants. These researchers found that unintended consequences flowed from their action (or inaction) that they were unable to predict - a warning to the inexperienced action researcher. McKay and Marshall provide similar cautions, and discuss the 'irremovability' of an information system from the surrounding context (2007; p. 133).

Within this research, each participant organisation was a unique client structure within which the action research cycle was played out. Throughout the remainder of the thesis the participant organisations are labelled Associated Counselling (AC) and Counselling Incorporated $(\mathrm{CI})^{5}$ and are introduced here as the contexts within which each action research cycle took place. Table 4 provides an overview of both organisations, and additional information about each is also provided.

\footnotetext{
${ }^{5}$ Associated Counselling and Counselling Incorporated are fictitious names used within this study to protect the identities of participant organisations.
} 


\begin{tabular}{|c|c|c|}
\hline & CI & $\mathbf{A C}$ \\
\hline Classification & SME & SME \\
\hline Established & 1949 & 1999 \\
\hline Core Service & $\begin{array}{l}\text { A wide range of relationship } \\
\text { counselling and education } \\
\text { services }\end{array}$ & $\begin{array}{l}\text { Specialised counselling } \\
\text { service }\end{array}$ \\
\hline Key Driver & Not-for-profit & For-profit \\
\hline $\begin{array}{l}\text { Geographical } \\
\text { Spread }\end{array}$ & $\begin{array}{l}\text { All of NZ, } 19 \text { regions } \\
\text { over } 70 \text { communities }\end{array}$ & $\begin{array}{l}\text { South Island, } 4 \text { regions } \\
\text { approximately } 15 \\
\text { communities }\end{array}$ \\
\hline Staff & 185 (101 FTE) & 11 staff \\
\hline Management & National office, 13 staff & Management team of four \\
\hline Target audience & $\begin{array}{l}\text { All community members, } \\
\text { including workplaces }\end{array}$ & Youth focused (aged 13-19) \\
\hline
\end{tabular}

Table 4: Summary of Participant Organisations

\section{Associated Counselling}

$\mathrm{AC}$ was, at the time of this study, a relatively new organisation still in the start-up phase. The organisation was founded when the developers of the particular counselling programme moved forward and commercialised it, forming AC. Although AC is a private, commercial SME driven by profit (among many other) motives, it had not yet returned noteworthy profit. At the time of this research, AC was comprised of four regional teams spread across the South Island with a total of 11 staff (a mix of full and part time work arrangements). Each staff member is a professional counselling practitioner, with some holding specialist skills and expertise, for example in clinical psychotherapy.

AC delivers a highly specialised and individualised programme to at risk youth in the regions they operate in. This programme broadly consists of a robust and proven counselling programme with a wilderness therapy component called the 'journey' woven into the programme. Wilderness therapy/counselling is a methodical, planned and systematic approach to working with troubled youth that involves skilled 
professionals guiding clients through both individual and group processes with activities aimed at creating changes in targeted behaviours, including clinical assessment, individual treatment plans, and participation in outdoor adventure pursuits (Russell, 2001). Most counselling sessions are held face-to-face, although some occur via telephone or other means as required.

With a management team of four experienced and highly professional clinical counsellors and psychotherapists $\mathrm{AC}$ has experienced organisational growth and begun to consolidate their internal process, policies and infrastructure.

\section{Counselling Incorporated}

$\mathrm{CI}$ is a N4P organisation that has a long history of providing relationship counselling services in communities nationwide. They have well-established organisational processes, policies and service offerings that provide a stable foundation for ongoing organisational activity. CI is geographically distributed across 19 regions and their 185 counsellors (many of whom work part-time) deliver a wide variety of relationship and workplace counselling and education programmes in large cities, towns and rural communities throughout NZ. Among their staff are specialist Maori community counsellors, and the services they provide (mostly in Far North communities) distinguish CI as a leader among other counselling organisations.

A strong team of 13 executive and management staff effectively manage a multimillion dollar budget and work with support staff to administer the clinical and operational activities from CI's centrally located Head Office.

The specific actions and key events that occurred at action research stages within AC and CI are summarised in Table 5. 


\section{AR Stage Definition of Action at Stages}

Diagnosing - Identify key problems that are the organisational drivers of change

- Consider the nature of the problem and the organisational domain

- Outline the theoretical framework and theoretical assumptions (Baskerville \& Pries-Heje, 1999)

\begin{tabular}{|l|l}
\hline $\begin{array}{l}\text { Action } \\
\text { Planning }\end{array}$ & $\begin{array}{l}\text { Specify organisational actions that } \\
\text { should be taken to relieve or improve } \\
\text { the identified problem (Baskerville } \\
\text { \& Pries-Heje, 1999; Lau, 1999) } \\
\text { Indicate the 'target for change' - the } \\
\text { desired outcome for the organisation } \\
\text { (Baskerville \& Wood-Harper, 1996) }\end{array}$ \\
- Indicate the 'approach to change' - \\
the changes that would achieve this \\
outcome (Baskerville \& Wood- \\
Harper, 1996)
\end{tabular}

\section{Key Events at AC}

- Initial contact with key participant

- Prepared project proposal for AC

- Participated in organisation-wide meeting

- raised awareness of and support for project

- facilitated discussion on the problem situation and intended actions

- Analysed current state of peer consulting at AC

- Defined target for change

- further defined problem situation and specified outcomes to pursue

- Explored use of ICT and infrastructure

- Assessed the potential of ICT to support peer consulting

- $\quad$ applied Theory of Media Synchronicity

- Selected and set up online systems

- Prepared and distributed written training guides

- Delivered systems training sessions via phone and online

- Initiated systems familiarisation period Developed the approach to change

- outlined the structure of a series of peer consulting sessions, including frequency, duration, topics, likely participants etc

\section{Key Events at CI}

- Initial contact with 2 key participants

- Developed and presented project scoping document and gained organisational support

- Raised awareness of project through national newsletter and attended national meeting

- Mapped peer consulting from two perspectives

- Assessed current peer consulting issues faced

- Presented findings to key participants

- Prepared and distributed business report, gained sign off from CI

- $\quad$ Explored use of ICT within CI

- Assessed the potential of ICT to support peer consulting

- applied MST

- Presented systems options to key participants

- Selected and set up online systems

- Delivered individual, face-to-face systems training sessions and prepared and distributed written training guides

- Initiated systems experimentation period ( $\mathrm{ad}$ hoc systems use)

- Defined target for change and desired organisational and research outcomes

- Developed the approach to change

- a set of sessions with predetermined topics, durations and desired outcomes 


\begin{tabular}{|c|c|c|c|}
\hline AR Stage & Definition of Action at Stages & Key Events at AC & Key Events at CI \\
\hline $\begin{array}{l}\text { Action } \\
\text { Taking }\end{array}$ & $\begin{array}{l}\text { Researcher and participants } \\
\text { collaborate to implement the planned } \\
\text { actions } \\
\text { Changes are made within the } \\
\text { organisation relevant to the target for } \\
\text { change (Baskerville \& Wood- } \\
\text { Harper, 1996) }\end{array}$ & $\begin{array}{l}\text { - Implemented the approach to change: } \\
\text { - } \quad \text { initiated peer consulting sessions 1- } 4 \\
\text { - } \text { provided technical and process support } \\
\text { - } \quad \text { communicated learnings } \\
\text { - Amended the approach to change as sessions } \\
\text { progressed } \\
\text { - Attended organisation-wide meeting to present } \\
\text { emergent outcomes and discuss general ideas and } \\
\text { experiences } \\
\text { Gained feedback on participant experiences, } \\
\text { outcomes and ideas and amended documents } \\
\text { accordingly }\end{array}$ & $\begin{array}{l}\text { - } \quad \text { Further defined the approach to change } \\
\text { - } \quad \text { fmplemented the approach to change } \\
\text { - } \quad \text { initiated peer consulting introduced online systems and series of } \\
\text { - } \quad \text { provided technical and process support } \\
\text { - } \quad \text { Supported continued ad hoc peer consulting } \\
\text { - } \text { Assessed outcomes of session } 1 \\
\text { - } \quad \text { Presented key findings to participants } \\
\text { - } \quad \text { Explored opportunity for implementing the } \\
\text { approach to change with participant group } 2\end{array}$ \\
\hline $\begin{array}{l}\text { Evaluating } \\
\text { Outcomes }\end{array}$ & $\begin{array}{l}\text { Assess whether the outcomes of the } \\
\text { change intervention were or were not } \\
\text { successful, from both a practical and } \\
\text { a research perspective (Baskerville } \\
\text { \& Pries-Heje, 1999) } \\
\text { - Conduct data collection activities }\end{array}$ & $\begin{array}{l}\text { - Assessed outcomes of sessions and ad hoc } \\
\text { consulting against target for change and research } \\
\text { objectives } \\
\text { - } \quad \text { Identified key points of value experienced by } \\
\text { participants } \\
\text { - Developed emergent themes } \\
\text { - Presented initial results to key participants }\end{array}$ & $\begin{array}{l}\text { - Assessed outcomes of session and ongoing ad hoc } \\
\text { online peer consulting against target for change and } \\
\text { research objectives } \\
\text { - Collated and distributed initial results to key } \\
\text { participants } \\
\text { - Attended national meeting to gain further feedback } \\
\text { from participants }\end{array}$ \\
\hline $\begin{array}{l}\text { Specifying } \\
\text { Learning }\end{array}$ & $\begin{array}{l}\text { Assess, document and distribute } \\
\text { knowledge and experience gained } \\
\text { throughout the action research cycle, } \\
\text { "reflection upon the results of the } \\
\text { strategic action plan to make sense } \\
\text { of the processes, problems, issues, } \\
\text { and constraints" (Rock \& Levin } \\
2002 ; \text { p. } 8 \text { ) }\end{array}$ & $\begin{array}{l}\text { Analysed the nature of online peer consulting at } \\
\text { AC } \\
\text { - Developed theoretical model of AC's experience } \\
\text { of introducing online peer consulting } \\
\text { - Presented findings to participants }\end{array}$ & $\begin{array}{l}\text { - Analysed the nature of online peer consulting at CI } \\
\text { - Developed theoretical model of CI's experience of } \\
\text { introducing online peer consulting }\end{array}$ \\
\hline
\end{tabular}

Table 5: Key Events at Action Research Stages 


\subsection{Role of the Action Researcher}

Chaisson \& Dexter (2001) state that a researcher is not just a facilitator or an interpreter, but also an agent for social progress and a change catalyst. Further to this, Baskerville \& Pries-Heje (1999) state that during an action research study researchers not only observe phenomena, they also intervene and participate in the situation under study. McKay \& Marshall (2001) agree, arguing that "the active and deliberate self-involvement of the researcher in the context of their investigation" is one distinguishing feature of action research (p. 47). As the structure of the action research process rests heavily on the roles of the researchers and research participants of the organisation in which change is being introduced, particular attention must be paid to the researcher role.

\begin{tabular}{|c|c|}
\hline $\begin{array}{l}\text { Researcher } \\
\text { Dominated }\end{array}$ & $\begin{array}{l}\text { Collaboratively } \\
\text { Managed }\end{array}$ \\
\hline \multicolumn{2}{|c|}{ ㄱon } \\
\hline Researcher model accepted & Model jointly developed \\
\hline $\begin{array}{l}\text { Researcher generated } \\
\text { information used }\end{array}$ & $\begin{array}{l}\text { Jointly generated } \\
\text { information used }\end{array}$ \\
\hline $\begin{array}{l}\text { Researcher makes key } \\
\text { decisions regarding AR } \\
\text { process }\end{array}$ & $\begin{array}{l}\text { Researcher/ system } \\
\text { Members make joint } \\
\text { decisions regarding AR } \\
\text { process }\end{array}$ \\
\hline
\end{tabular}

Figure 3: Researcher Role in Action Research (Chisholm \& Elden, 1993; p. 287).

Different action research types and each different organisational situation will necessitate a different researcher role (see Figure 3). I acknowledge the importance of collaboration with the research participants in my study (see below), however, as I was solely responsible for identifying possible technology and process solutions to relieve the problem situation, analysing outcomes of the change intervention and managing practical and research project activities I identify my primary researcher role as aligning more closely with the researcher-dominated end of the continuum (Chisholm \& Elden, 1993).

Examining the nature of researcher roles in more depth, Chaisson \& Dexter (2001) offer three types of typical researcher involvement, collaborative, facilitative and 
expert as outlined in Table 6. As I led all aspects of research design and collaborated with participants from both organisations on aspects of the change interventions, my role more closely resembled that expert, not the facilitative or collaborative researcher.

\section{Researcher Role}

Collaborative

\section{Facilitative}

\section{Expert}

Table 6: Types of Researcher Role

\section{Role Definition}

- Collaboration is recognised as an important part of the research design

- Researcher and participants discover together how a system/ innovation functions

- Uses the tacit and explicit knowledge of insiders plus the formal frameworks and concepts of outside researchers (Chisholm \& Elden, 1993)

- $\quad$ Typically distinguishes the researcher as an 'expert' among study subjects.

- $\quad$ Research activities may be undertaken cooperatively between researcher and participants

- $\quad$ Tasks of researcher and subjects remain quite distinct (Baskerville \& Wood-Harper, 1998)

- $\quad$ The researcher brings general knowledge of systems, social science, and the research process

- Organisational members bring in-depth understanding of their system, how individuals perceive phenomena, and how to 'get things done' (Chisholm \& Elden, 1993)

- The 'burden' of solving the immediate organisational problem rests with the research participants

- $\quad$ Researcher facilitates/ helps participants by offering expert advice, technical knowledge or an independent viewpoint (Baskerville \& Wood-Harper, 1998)

- May result from the assumption that both outside researchers and organisational members have crucial contributions to make to the research situation (Chaisson \& Dexter, 2001).

- Distinguishes the researcher as an expert among study subjects (Chaisson \& Dexter, 2001)

- Involves cooperation and distinct tasks (as for 'facilitative' role), but the burden of solving the immediate organisational problem rests with the researcher

- The researcher's decisions may determine the interventions to be made (Baskerville \& Wood-Harper, 1998)

- Researcher maintains control over most, if not all, critical aspects of the action research process

- Minimal participant roles generally occur 


\subsubsection{Researcher role at action research stages}

The specific researcher and participant roles that were undertaken at the action research stages of diagnosing, action planning, action taking, evaluating outcomes and specifying learning are summarised in Table 7 . These are the general activities that were undertaken within each participant organisation, and as the context, identified problem and necessary change intervention varied slightly between the two participant organisations, there were also slight differences in my roles.

\section{AR Stage}

Diagnosing

Action

Planning

Action

Taking

Evaluating

Outcomes

Specifying

Learning

\section{Researcher Role}

- Entered organisations

- Convened participant advisory boards

- Collaborated with participants to define organisational problem

- Researched: conducted interviews, gathered documentation, observed organisational norms and participant actions, conducted initial analysis

- Collaborated with participants to specify necessary problem-solving actions

- Advised participants on needed ICT and processes

- Communicated proposed interventions across organisations

- Introduced ICT and facilitated its early use

- Researched: conducted interviews, gathered documentation, observed participant actions, analysed data

- Collaborated with participants to initiate action

- Facilitated peer consulting sessions

- Researched: conducted interviews, gathered documentation, observed participant actions, monitored systems statistics, analysed data, developed themes

- Evaluated outcomes of change intervention against research questions and target for change in each organisation

- Researched: conducted interviews, analysed data, developed model

- Researched: conducted interviews, analysed data, refined model

- Assessed research outcomes

- Formalised the learning that took place:

- presented findings to the participant organisations

- gathered feedback on findings

Table 7: Summary of Researcher Role at Action Research Stages 
Whichever action research type is chosen and particular researcher role undertaken, Morrison \& Lilford (2001) advise that the values held by each of the involved parties are made explicit and that attempts are made to arrive at a specific 'values basis' for the research project. In order to ensure that there was congruence between the values, expected actions and desired actions a proposal or scoping document was prepared and presented to each participant group. Mumford (1999) warns the action researcher to remember that they are operating in "volatile political situations where there may be different, even hidden, agendas" (Mumford, 1999; p. 20) and suggests that the researcher and participants create a listing of what is to take place during the research period, perhaps in a formal document such as I have done.

\subsubsection{Collaboration within action research}

One key aspect of action research is its collaborative nature whereby the researchers work closely with participants who provide the subject system knowledge and insight necessary to understand the problem situation being studied (Baskerville \& WoodHarper, 1996). A useful example is the research of Harris \& Harris (2002) that explored the possibilities for organisational collaboration between eight voluntary organisations in the HIV/AIDS area. A feature of their research was that the work was to be carried out by the researchers in collaboration with the executives who had commissioned it. A recent study by Ercetin (2002) employed a higher level of collaboration and used collaborative participation, whereby people work together as equals, engage in negotiations and develop situations. Similarly, McKay \& Marshall (2001) stated that collaboration was one of four distinguishing features of action research and involved a form of mutual dependence where each party "relies on the others skill, experiences and competencies to achieve the dual goals of action research" (p. 47).

When undertaking information systems research, collaboration between the researcher and research participants has been proven essential. In an action research study into systems design, Mumford (1999) found that a collaborative approach was needed as future system users from differing levels of the client organisation could 
contribute significantly to organising the new work systems. Relatedly, Leh (2002) conducted an action research intervention to introduce teachers on a graduate level technology course to technology advancements. This researcher employed a collaborative approach and worked with research participants to overcome a knowledge gap and generate new knowledge:

"Technology integration requires knowledge of subject
areas in addition to technical expertise. The author
could easily integrate technology into one subject area,
such as second (foreign) language learning, but she did
not feel confident in effectively integrating technology
into another subject area, for example, science. As a
result, she constructed knowledge together with her
students who were experts of their own subject areas"
(Leh, 2002; p. 45).

More recently, Nosek (2007) emphasised the importance of the client-researcher relationship and advocated for enabling those within organisations, previously research participants, to take on more of a research-focused role.

While I did not empower research participants to assume the role of researcher at any stage, my study, as in the examples discussed, did employ a degree of collaboration with organisational members. As I am not a subject matter expert in counselling services, I needed to collaborate closely with research participants in order to gain the subject area knowledge and insight necessary to understand the problem situation under study, introduce a technological change to alleviate the identified problem and achieve the problem solving goals of this research.

\subsection{Data Collection Procedures}

Action research often takes a process view of research that requires sustained data collection over a long period of time. "Since action research is change oriented, it requires data that help track the consequences of intended changes. So, action research must have data collected systematically over time" (Elden \& Chisholm, 1993; p. 128). Through various intervention cycles that allow the researcher to continuously reconsider the problem being faced and researched, action research can 
enable researchers to elicit detailed and context-rich descriptions and to ensure that the learning obtained during interventions is carefully considered (Davison, 2001). As action research takes place in a unique organisational context, the researcher is exposed to the complexities and richness of the situation under study.

Miles \& Huberman (1984) recognised that there exists sparse standardised instrumentation appropriate for qualitative data collection. Action research does not prescribe specific data collection activities, and there do not need to be restrictions placed on the methods chosen. I have thus turned to previous action research studies and examined the data gathering procedures employed. Some examples are included in Table 8.

\section{Researchers}

\section{Paper Title}

Knowledge integration in

Tanudidjaja, Kankanhalli \& Tan, 2003

Harris \& Harris, 2002

Barker-Ruchti, 2002

Lau, 1997 global virtual teams.

Achieving organisational collaboration in the nonprofit sector: An action research approach.

A study journey: A useful example of action research.

A Review on the Use of Action Research in Information Systems Studies.

\section{Data Collection Procedures}

Observation and transcripts of meetings, communication logs (eg: emails or web board postings), and project documentation.

Document examination; semistructured one-to-one interviews with participants internal and external to the organisation; group discussions, and written reports and other literature examination.

Individual interviews, group interviews, and questionnaires.

Interviews, participant observation, questionnaires, focus groups, site visits, field notes, document review and video recording.

Table 8: Data Collection Procedures in IS Studies 
Whichever particular data collection methods are chosen, from the above listing it is apparent that multiple data collection methods are necessary to complement the strengths and compensate for the limitations of each.

In this study, I have applied a range of data collection techniques, as appropriate at different stages of the action research cycle. Table 9 summarises these techniques, and a more detailed discussion of the two primary data collection techniques, cases and interviews, follows.

\section{AR stage}

Diagnosing

\begin{tabular}{|l|}
\hline \\
\hline Action \\
\hline Planning
\end{tabular}

Action

Taking

Evaluating

Outcomes

Specifying Learning

\section{Data Collection Techniques- AC}

2 interviews (via phone), meeting notes, observation notes, journal entries, participant feedback, organisational documents

3 Interviews (1 face-to-face and 2 phone), feedback from advisory board, journal entries, feedback through training sessions, systems statistics, written feedback via email

14 interviews (4 face-to-face and 10 via phone), systems statistics, feedback on sessions (via phone and email), feedback from advisory board, feedback via organisational meeting, observation notes, journal entries

\section{1 interview (face-to-face) and 1} group interview (5 participants, face-to-face), feedback via organisational meeting, journal entries

Organisational documents, journal entries, feedback from advisory board

\section{Data Collection Techniques- CI}

5 interviews (face-to-face), organisational documents, meeting notes, journal entries, notes from informal feedback

2 interviews (face-to-face), feedback on systems selected and process outlined, feedback from advisory board, journal entries, feedback through training sessions, systems statistics

12 interviews (7 face-to-face, 5 via phone), systems statistics, feedback on sessions (via phone and email), feedback from advisory board, feedback on initial findings, journal entries

2 interviews (face-to-face) feedback via organisational meeting, journal entries

Feedback from advisory board, journal entries

Table 9: Data Collection Procedures at AR Stages 


\subsubsection{Case study}

Case study has been identified as a common form of reporting information systems research that enables a researcher to explore and explain the causal links in real-life interventions and effects (Lau, 1997). Within the action research method, each organisational situation has been written up as a separate explanatory case. This helped to refine large amounts of data and reveal any themes or patterns. Miles and Huberman (1984) define a case as "a phenomenon of some sort occurring in a bounded context" (p. 25).

Braa \& Vidgen (1999) distinguish between two types of case study: the positivistinformed hard case study and the interpretivist soft case study. Hard case study is generally that defined by Yin (1994), as "an empirical inquiry that investigates a contemporary phenomenon within its real-life context, especially when the boundaries between phenomenon and context are not clearly evident” (p. 13). In contrast, soft case study, with an interpretivist approach, is concerned with gaining understanding and subsequently generalising to 'the social totality' beyond each individual case (Braa \& Vidgen, 1999).

This research has followed the soft case study approach, and the story of each organisation has been written from the point of view of key decision makers at AC and CI, describing events including facts about the roles of participants, their opinions and perceptions of technology use and any additional relevant background information (see Chapters $5 \& 6$, respectively).

\subsubsection{Interviews}

Interviewing was the primary data collection activity of this study. Through semistructured interviews, data was collected throughout the action research cycle in the form of words. This technique has been used in the information systems discipline to describe a behaviour or set of behaviours in a focal person (Bostrom \& Clawson, 
1992). Through semi-structured, face-to-face interviews, I asked respondents to recall actual occurrences of specific events, actions and behaviours that they undertook as part of their organisational role. Through these interviews I also gathered relevant demographic information, an overview of the current organisational situation faced by each participant, with the focus of interviews being on the descriptions of work practices and roles and the participants' perceptions of participating in online peer consulting sessions.

Over a period of two years, each research participant was involved in multiple interviews at different stages of each action research cycle, totalling a set of 43 unique transcripts. I conducted multiple interviews as a single, brief interview with each participant would not have provided sufficient in-depth and clear insight into their actions and experiences at various stages of the study. I have termed these interviews semi-structured as a set of guiding questions was employed. However, the interviews were not limited to a particular set of questions and the interviews followed a conversational manner within which participants freely offered comments on their experiences gained during the project. Interviews were therefore semistructured, as I was able to focus them without overly limiting participants' answers.

All interviews were recorded and transcribed soon after each interview was completed. As the volume of data collected grew I found it necessary to use the NVivo software tool to organize the data. This helped to ensure that I could undertake initial analysis at the Diagnosing stage (see below) within each organisational situation, and also ensured that all data collected remained searchable, discoverable and able to be further analysed at later stages of the action research cycle. The data analysis techniques that I have applied are described in the next section. 


\subsection{Data Analysis Techniques}

Taking a holistic view of the five action research phases, the problem situation is usually tackled more precisely with each completed cycle. In this way, problems can be investigated rigorously and relevant and practical results can emerge from the analysis. However, "action research methods may be refined to improve their ability to contribute rigorously to information systems research" (Baskerville \& Pries-Heje, 1999 ; p. 2). These authors combined grounded theory data collection and analysis techniques to the phases of the action research cycle to add more rigour to the theory formulation component of their action research study. Following this example, I have chosen specific data analysis techniques of coding and linking of codes to various aspects of a conceptual framework in the first and subsequent analysis stages, as detailed by Yin (1994) and Miles \& Huberman (1984).

Through transcription of interviews the data collected was refined into text conducive to analysis (Miles \& Huberman, 1984). Unstructured preliminary analysis of assigning data meaning took place as transcription occurs. Miles \& Huberman (1984) describe qualitative data analysis as consisting of concurrent flows of activity whereby data collection and analysis is a cyclical process, which aligns with the overarching action research methodology, where a critical activity is interpreting and making sense from the collected and refined data. I have used various methods to undertake this sense-making, as summarised in Table 10. Two methods of coding and template coding are described further below. 


\section{AR stage}

Data Analysis Techniques- AC

Defining

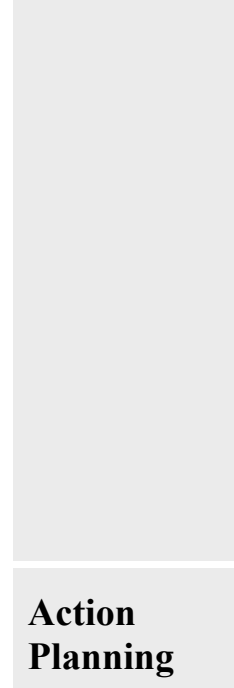

- Link codes to Media Synchronicity Theory (MST)

- Code interview transcripts with MST codes list

- Identify themes and write case

\begin{tabular}{|l|}
\hline $\begin{array}{l}\text { Action } \\
\text { Taking }\end{array}$ \\
\hline \\
\hline $\begin{array}{l}\text { Evaluating } \\
\text { Outcomes }\end{array}$ \\
\hline
\end{tabular}

- Analyse all data with refined PC codes list (v2)

- Open coding of interview transcripts to reveal additional themes

- Analyse data with PC codes list (v3)

- Develop AC Model of Peer Consulting Transition

- Organise data within model

Specifying Learning

- Analyse all data with refined Technology-in-Practice (TiP) codes list

- Refine AC Model of Peer Consulting Transition

\section{Data Analysis Techniques- CI}

- Open coding of interview transcripts

- develop 'shopping list' of codes

- organise into categories

- write memos of themes

- Link codes to peer consulting process template

- Analyse data with PC codes list (v1)

- Write case and cross-case comparison

- Link codes to MST

- Code interview transcripts MST codes list

- Identify themes and write case

- Analyse all data with refined PC codes list (v2)

- Open coding of interview transcripts to reveal additional themes

- Analyse data with PC codes list (v3)

- Develop CI Model of Peer Consulting Transition

- Organise data within model

- Open coding of interview transcripts

- develop 'shopping list' of codes related to TiP framework

- Analyse all data with refined TiP codes list

- Refine CI Model of Peer Consulting Transition

Table 10: Data Analysis Procedures at AR Stages 


\subsubsection{Coding}

Firstly, I coded the data by noting points of interest in the margins of the transcriptions (see Appendix B for an example of a codes list). Each code was a label that assigned meaning to a section of data that I found significant (Miles \& Huberman, 1984). The notations, or codes, were a summary of a section of data or a key word or words that I marked for future consideration and further analysis. As I was be dealing with a large amount of textual data from multiple sources and different stages of the action research cycle, reducing and refining data early on in this study was important. Yin (1994) terms such a procedure pattern matching and states that it is a method of increasing the internal validity, or the accuracy of the information and the extent to which it maps real life events.

The collection and ordering of data within the early stages of the action research cycle resulted in a repository of participant actions and experiences from which I drew themes that were then used for further data analysis at the Evaluating Outcomes and Specifying Learning stages. An action research intervention usually allows participants to have opportunities to be affected by and influence the study outcomes (Cockburn \& Trentham, 2002). In keeping with this, I presented the themes emergent in the data to key participants as initial documentation on which to reflect and check for accuracy.

\subsubsection{Template coding}

In their study of knowledge integration in global virtual teams, Tanudidjaja et al. (2003) applied King's (1998) template coding data analysis approach. Following this approach, the researchers produce a list of codes, the 'template', representing themes that were defined a priori. However, the codes may be modified or added to as the researcher studies and interprets the data. A researcher may asses the major categories or propositions of their guiding theory and then incorporates categories and/or sub-categories based on the literature reviewed as part of the study. The resultant template will include all categories and sub-categories in a form that is 
useful to the researcher, perhaps a simple table. In their analysis, Tanudidjaja et al. allowed the preliminary pattern of the organisational case to emerge by coding the original template using their various data sources. Next, they identified and coded individual incidents in their transcripts. After analysis activities were complete, the original template was reviewed based on the data analysis and the final template was derived through iterations. Following the template revision, the data was re-analysed to identify relationships between the categories/sub-categories using axial coding procedures of Strauss \& Corbin (1990).

As Table 10 shows, at the Diagnosing, Evaluating Outcomes and Specifying Learning stages of the action research cycle I developed templates of codes that were the tools with which I analysed data:

- Diagnosing stage: template coding enabled me to accurately map the peer consulting process at $\mathrm{CI}$ and gain an overview of the nature of peer consulting activities at $\mathrm{AC}$

- Action Planning stage: by creating a template from core elements of the Media Synchronicity Theory I was able to code interview transcripts and organisational documentation to identify the types of media best able to support the communications processes within each organisation

- Evaluating Outcomes stage: I was able to organise emergent themes into categories and subsequent activities from which I created a template that I used to analyse all data collected, and eventually developed the Model of Peer Consulting Transition

- Specialising Learning stage: I refined the model and the coding template and conducted further analysis on interview transcripts and written feedback. I also used elements of the Technology in Practice framework to develop an analysis template that I used to analyse all data and identify important aspects of participants' use of technology to support peer consulting activities.

The concurrent nature of the data collection and analysis that I undertook enabled me to refine the coding 'shopping lists' and revisit data as necessary throughout the stages of the action research cycle. McKay and Marshall (2007) describe this as the "constant, iterative decision making" that exists in action research studies. This was instrumental in enabling me to develop a model that reflected the various actions taken by participants as they undertook the transition from traditional, face-to-face 
peer consulting to include online peer consulting. Identifying emergent themes in the Acting Taking stage and developing the model early in the Evaluating Outcomes stage enabled me to gain feedback from participants of both organisations, which I used to inform the final version of this model.

\subsection{Chapter Summary}

Qualitative research methods are increasingly used in the information systems discipline as a way to generate valid research results that have high relevance to practice. The classical action research method with a moderate degree of participant collaboration is to be applied in this study, and complemented by specific data collection and analysis methods. I employed both observational and interview data collection methods to accurately capture participants' experiences and perceptions of the adoption and use of online resources for professional development activities. Data analysis leading to theory development was based around various open and template coding procedures and led to the development of a theoretical model and enabled important insights into the nature of participants' use of technology for peer consulting activities.

I believe that the data collection and analysis techniques that I have applied have contributed to a rigorous research process that has enabled me to relieve and shed light on an important organisational problem, while also contributing to information systems theory through the generation of a theoretical model. The following chapter outlines this model, and Chapters 5 and 6 tell the organisational stories of the development of an online peer consulting programme at $\mathrm{AC}$ and $\mathrm{CI}$. 


\section{Overview of the Model of Peer Consulting Transition}

\subsection{Overview}

In this chapter, I introduce the conceptual model that resulted from this study. As research into the use of CoPs for professional development remains emergent (Riverin \& Stacey, 2008), there are currently no models that provide an adequate roadmap to developing an online professional development programme. The model that I outline in this chapter and elaborate on through Chapters 5 and 6 goes some way to addressing this gap.

In developing the model, I analysed the actions of research participants as they moved through the process of transitioning from a traditional professional development programme to complementing this by trialling online professional development, or more specifically online peer consulting. Specifically, I gathered all data collected from CI during action research stages in the NVivo system, including: interview transcripts, meeting notes, organisational documents, emails, observation notes.

Once the data had been coded with the initial set of codes (see, for example Appendix B), I ran several reports and noted patterns emerging within the data, such as the occurrence of particular phrases, topics and issues. I organised the data into sets based on the identified patterns, and then developed some initial, high level categories that encompassed two or more related patterns, for example:

- Conceptualising vs operationalising online peer consulting: how and to what extent specific elements of an initial and/or evolving organisational online peer consulting concept have been put into action

- Influence of pre-existing relationships: the ways that participants draw on their relationships with peers before, during and after online peer consulting sessions to support professional learning 
- Decisions undertaken on appropriate ICT: aspects of the process that participants undertook when selecting, using and evaluating ICT tools to support online peer consulting, particularly changes in needs and improved understandings

- Inclusivity and flexibility - embedded in organisational culture: the ways that organisational values of inclusivity and flexibility translate to the online peer consulting sessions, supporting and/or encouraging participation and sharing of knowledge

- Tenacity vs technology: evidence of reasons why participants displayed such tenacity to continue online peer consulting, despite persistent ICT-related inhibitors

- Leadership of project, leadership of sessions: the nature and impact of activities (and/or behaviours and attitudes) of the project and peer consulting session leaders

- Lasting impressions - the spark of change?: any suggestion and/or evidence that elements of the experience of transitioning peer consulting to the online environment - most importantly participating in sessions - resulted in lasting change to organisational professional development practices

At this point, I paused my analysis efforts and wrote a description of each potential category, which further revealed the relationships between and within categories. Subsequently, some categories were merged, some split and I reorganised data within the revised categories. On completion of this reorganisation I had generated five core categories, each having two or more sub categories. Further reorganising of these categories led to the development of a model within which the actions of research participants fall under five stages that together form the Model of Peer Consulting Transition, as depicted in Figure 4 below. 


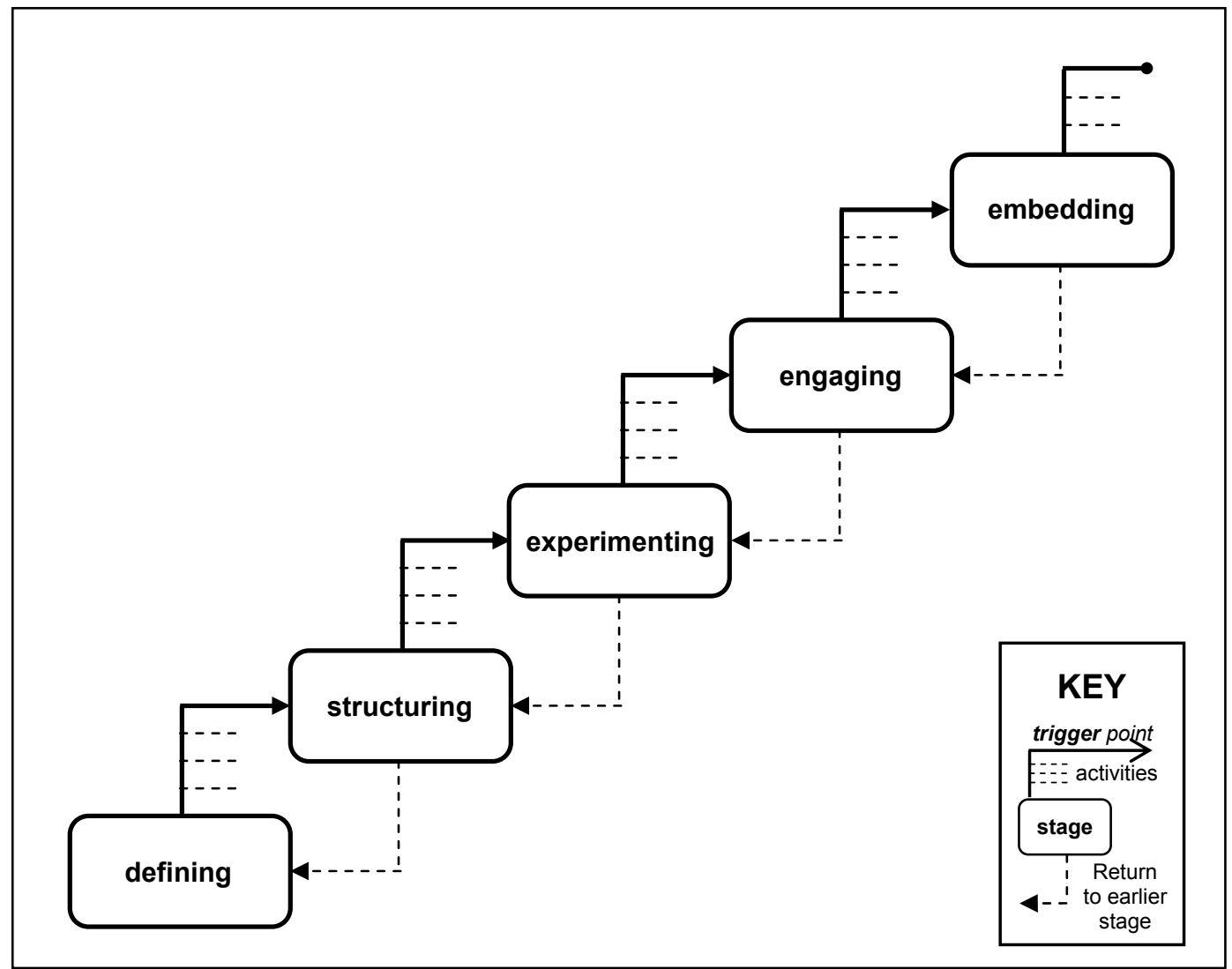

Figure 4: Stages of Peer Consulting Transition

A staged model emerged as particularly appropriate for this study. In Chapter 2 I positioned each group of participants as a learning community and explored the concepts of communities of practice (CoPs) and online CoPs. Further examination of the literature in this area reveals that there is an established history of the development and application of staged lifecycle and evolutionary models to describe the process of the formation, maturation and termination of CoPs. Specifically, I draw on the works of Wenger (1998), McDermott (2000) and Gongla and Rizzuto (2001) when describing elements of the stages of my model (see Appendix A for detail of community of practice frameworks).

Wenger (1998) postulated that, over time CoPs move through various stages of development (Potential, Coalescing, Active, Dispersed and Memorable) that are characterised by levels of interaction among members and the different kinds of activities that they undertake. Similarly, each stage of my model is characterised by a 
number of key activities that combine to make each stage distinct from others. While in practice particular activities may span across stages, or for various situational reasons be repeated at various stages, they have been assigned to the stage that I observed them to be most dominant within.

There is one important aspect of Wenger's model that my model does not share. The stages of Wenger's model build on each other and occur over a period of time, and as such Wenger asserted that it is not possible for a community of practice to move from the Active stage back to the earlier Coalescing stage, for example. Although my model includes stages that build on each other, and activities naturally are undertaken over a particular time period, there is scope for an organisation to return to the activities of earlier stages when necessary. In Figure 4 above, this movement is represented as the dotted line underneath each stage box. In congruence with the Community Evolution model developed by Gongla \& Rizzuto (2001), it is possible for a peer consulting community to reach its potential and 'dissolve' at the Experimenting, Refining or Embedding stages.

The final aspect of my model is a particular activity or point within each stage that was most prominent in propelling transition from one stage to the next. I have labelled such activities Trigger Points, as I observed them acting as the catalyst for an organisational group shifting from activities of one stage to engaging in activities of the next stage.

Within the remainder of this chapter I present each stage of the conceptual model, giving a brief overview of the key activities and trigger point of each stage. 


\subsection{Defining Stage}

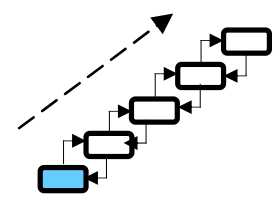

The objective of the Defining stage is to improve understanding of the current state of professional development, and in particular peer consulting practices, within the organisation. This is an important fist step in the process where current professional development practices, processes and capabilities are assessed. Through consideration of such elements an initial conceptualisation of online peer consulting is formed. I have organised the observed actions of group members at the Defining stage into three categories, represented as the activities of:

- Mapping current peer consulting activities

- Documenting current ICT support

- Developing the online peer consulting concept

\subsubsection{Mapping current peer consulting activities}

Peer consulting transition begins as a process of discovery, exploring the professional development practices, processes and capabilities within an organisation, with the objective of mapping current peer consulting activities. Through this exploration knowledge of any existing peer consulting practices is generated, and a picture of where practices fulfil or fall short of fulfilling the professional development needs of organisational members is formed. It is likely that people are facing similar issues, but without the benefit of a shared practice (Wenger, 1998). The knowledge of current peer consulting practices gained is used to indicate those practices that could benefit from being translated to the online space, or peer consulting practices currently absent that could be offered in an online format.

Assessing current peer consulting practices reveals the nature of the peer networks that exist, which Akkerman et al. (2008) label the shared history of community members. As Akkerman et al. note, such networks may be geographically based, and form "loosely connected networks based on doing similar work" (2008, p. 392). Communities of practice often emerge out of existing social networks, and the 
importance of a shared history, and its contribution to the 'social fabric' of learning, has been well documented (McDermott, 2000; Wenger, McDermott and Snyder, 2002). The extent of shared history is an important aspect of a peer consulting community, as the existence of shared history acts as a motive for shared activity, in this study peer consulting (Akkerman et al., 2008). Conversely, lack of shared history can result in a lack of willingness to collaborate, a fundamental function for peer consulting.

The key outcomes of this activity are a description of current peer consulting practices and peer consulting process mappings generated.

\subsubsection{Documenting current ICT support}

Once the current peer consulting practices are identified, the next task is documenting current ICT support of such practices. Here, the focus is on outlining the particular tools used to support peer consulting practices, as well as the ICT that is available within a particular organisation for a peer consulting community to use.

Highlighting the importance of technology support, Gongla and Rizzuto (2001) include 'enabling technology' as a fundamental function of at each stage of community evolution. Similarly, it is important that an assessment of ICT occurs early in my model, as an explicit focus on ICT allows a direct connection between programme development (and peer consulting transition in general) and ICT.

Current ICT support points are added to the peer consulting process mappings, presenting a fuller view of the current state of peer consulting within an organisation. Assessing the amended diagrams may also reveal the points at which there is potential to apply ICTs, and possibly also the communicative tasks that require support. 


\subsubsection{Developing the online peer consulting concept}

After consideration of the current peer consulting activities and corresponding technology support, the final activity of the Defining stage - developing the online peer consulting concept is undertaken. Based on information gathered thus far and the general culture/values of the organisation, an initial vision of what online peer consulting means for the wider organisation, and also for particular groups within the organisation, is developed. Part of developing the concept includes an assessment of who within an organisation already networks around a topic (McDermott, 2000), and how this may be extended through online peer consulting.

It is important that organisational members develop a strategic view of online peer consulting at the Defining stage, as at the Embedding stage the online peer consulting programme needs to integrate with, not replicate or replace, existing professional development activities. Focussing on the strategic-level concept will help an organisation to envisage how an online peer consulting community could add value to the individuals involved and the wider organisation (McDermott, 2000). McDermott states that this can be a catalyst for growth of and interest in the community.

It is likely that developing the online peer consulting concept will result in a form of formal or informal document, for example a vision or scoping document, that is disseminated to organisational sponsors and targeted peer consulting community members for discussion and feedback.

\subsubsection{Galvanising point}

Through the activities of the Defining stage an organisation gains a snapshot of their current peer consulting practices and ICT support. Also at this stage, an organisation takes the first tentative steps in developing an online peer consulting concept. Looking ahead at the opportunities for complementing current peer consulting 
practices with an online programme will make the potential for online peer consulting clearer. If this potential motivates key organisational members to introduce online peer consulting, then the organisation is ready to progress to the Structuring stage. This is termed the Galvanising Point as it is here that the organisation moves quite markedly from assessing what currently occurs within the organisation to furthering the initial concept by structuring a peer consulting programme around the newly developed concept.

\subsection{Structuring Stage}

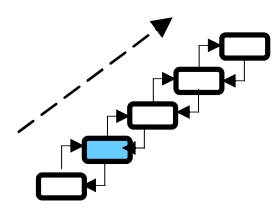

Creating the structure of a peer consulting programme involves planning for a series of peer consulting sessions, how they will be supported technically, and the capability of group members to participate in sessions. These are the operational aspects of how the shift from traditional professional development practices to online peer consulting will occur. Building on the Defining stage outcomes, the Structuring stage comprises several observable activities that I have organised into three groups, represented as the key activities of:

- Planning for a series of sessions

- Selecting and setting up systems

- Training on systems use

\subsubsection{Planning for a series of sessions}

In planning for a series of online peer consulting sessions, the knowledge generated in the Defining stage is applied to create an initial schedule for the online peer consulting programme. This includes the nature of the series of online peer consulting sessions, possible consulting topics, session time frames, session resources and potential outputs. It is likely that a schedule for systems selection, development and training, and other resource needs will be assessed and planned for at this stage. Akkerman et al. (2008) describe additional coordinative tasks that I include as part of this activity, such as setting a meaningful direction for sessions, 
planning for the necessary space and time to discuss session objectives and organising community building activities.

It is important that all elements of the desired sessions are addressed at this stage, as decisions taken at the Structuring stage affect the nature of engagement at later stages. For example, by structuring sessions that are closed to people outside the peer consulting community, regular, on related issues and follow a similar structure it is more likely that greater continuity of interaction will exist at the Engaging stage, and that peer consulting community members will begin to develop some shared understandings and approaches to their practice (McDermott \& Snyder, 2002). Therefore, a plan for a series of sessions is likely to focus on the use of online tools for a series of structured peer consulting episodes, complemented by informal, or ad hoc peer consulting between structured sessions.

Planning the series of sessions culminates in a document (formal or informal) outlining a series of online peer consulting sessions that may be disseminated to organisational members or groups. Planning activities may lead to a clearer view of what online peer consulting can look like for the organisation, and for a particular peer consulting community, thus extending the vision and concept of online peer consulting developed at the Defining stage.

\subsubsection{Selecting and setting up systems}

Selecting and setting up systems to support online peer consulting is the next challenge in the process to developing a peer consulting programme. This is an important activity within the Structuring stage, as without adequate ICT support an organisation cannot progress further. As Riverin and Stacey (2008) highlighted, "information and communication technologies have encouraged the creation of rich online environments where participants can share professional strategies, examples of best practice, and professional development opportunities" (p. 43). However, the process by which organisations select and apply various technologies for 
professional development activities, which Akkerman et al. (2008) class as a coordinative activity, remains largely unexplored.

Determining the kind of ICT support that online peer consulting requires involves assessing the process mapping(s) generated at the Defining stage, as well as the newly developed plan for a series of sessions. Process mappings will show the types of communicative tasks that need to be supported, the types of resources that are exchanged, whether peer consulting is synchronous or asynchronous, and how many peers are typically involved. These elements all inform the decisions of which ICT tools are required to support peer consulting.

Often, the needed ICT resources are not available within the organisation, or if they are available, they need to be reconfigured to adequately support peer consulting sessions. Additional factors that are likely to influence systems selection include budget and time constraints, as well as the target group's comfort and experience with online tools.

Following this assessment a list of potential systems is generated, from which the most appropriate is selected and then set up for use in training sessions.

\subsubsection{Training on systems use}

The initial use of systems will likely be in the form of training on systems use. As some members of the intended peer consulting community may not have the technical literacy needed and others may not have used the particular system or tools selected, training is a vital activity in preparing for engaging online peer consulting. In the study of Riverin \& Stacey (2008), many supporting systems involved a fairly simple learning curve, which contributed to the success of the learning communities. As described in the literature review, one key barrier to participation in online communities is the investment of time required. Time to learn a new system can be a significant barrier to the launch of an online community, and so training must be 
carefully planned and delivered to provide members with the skills needed to participate in initial peer consulting.

Training may take the form of face-to-face sessions, telephone sessions, be delivered primarily in written form or be a combination of various methods. Elements such as the culture of the organisation, the knowledge, skills and experience of the people involved, the complexity of the online tools and the time available for training determine the most effective training channel. A community should take advantage of any opportunities for face-to-face training, as this may provide community members with a chance to develop and/or strengthen relationships with peers, that can provide support at later stages of peer consulting transition, for example in coping with technological challenges (Riverin \& Stacey, 2008).

Through participating in systems training, community members will improve their capability to take part in online peer consulting. In addition, questions addressed during training sessions may help to improve understanding of the concept of online peer consulting, and the organisation's vision for introducing this initiative.

\subsubsection{Rousing point}

Taken together, the activities of the Structuring stage are intended to motivate the organisation and peer consulting community members to engage in online peer consulting. By preparing to engage in peer consulting through planning for sessions and introducing online tools to community members, both their understanding of the peer consulting concept, the potential value that online peer consulting can offer individuals the community and the wider organisation, and their confidence with the online tools is improved. The experience of planning for sessions is likely to have raised the interest in online peer consulting, and the training sessions are likely to have sparked people's interest, described by McDermott (2000) as the spike of interest and excitement that encourages a community to form. This is the Rousing 
Point that propels the organisation to the next stage, where newly developed capabilities are put into action to make initial online connections with peers.

\subsection{Experimenting Stage}

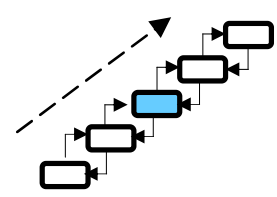

The objective of the Experimenting stage is to build on the Defining and

Structuring stage outcomes and put the online peer consulting concept into action.

This involves actioning the first phase of the previously developed plan, and putting into practice the newly developed or extended capabilities of the peer consulting community. Here, the community uses the online system to support their communication on practice related issues, in an informal manner. Based on this experience the conceptualisation of online peer consulting can be further developed, with the possible flow on effect of changes to both the structure of the series of sessions, and the online tools. I have organised the various observed tasks of the Experimenting stage into the three broad categories of:

- Participating in informal online peer consulting

- Altering the plan for sessions

- Reconfiguring systems

\subsubsection{Participating in informal online peer consulting}

In the Experimenting stage, the peer consulting community has the first opportunity to participate in informal, online peer consulting and build on the experiences gained in the training sessions and gain first hand experience of online peer consulting. They can test out the online system by using the various tools exchange practice related ideas with one another, in an informal and ad hoc manner. Through these initial exchanges, individuals are likely to discover commonalities with their peers (Wenger, 1998), which ultimately helps to build a shared history among peer consulting community members (Akkerman et al. 2008). This experimentation typically takes place during a predetermined time period, to ensure a clear distinction between informal and formal peer consulting activities. 
It is important to note that at the Experimenting stage 'lurking' - choosing to remain on the periphery of activity and observe others participating more actively in peer consulting exchanges - is a legitimate form of participation (Wenger, 1998). There are many reasons that community members may prefer to remain 'invisible' at this early stage of transition, and they are still able to derive value from observing others (Wenger, 1998). Indeed, Riverin and Stacey (2008) noted that a successful community includes a balance between lurkers and contributors, which may change as the community matures.

One challenge the organisation faces at this stage is providing the right "balance between management directives and user experimentation for fostering creative incorporation and application of the technology in the user's day" (Munkvold, 2002; p. 363). Thus, an experimental, rather than strategic approach to participating in informal, online peer consulting needs to be taken here (Chau \& Turner, 2002). This may be a challenge to some organisations that do not have a culture that is supportive of experimentation, as Riverin \& Stacey (2008) found in some of their research cases.

Shifting from participating in training sessions to informal peer consulting evolves use of the online tools and subsequently also improves the capability of individuals to participate in formal online peer consulting sessions. Following the period of informal peer consulting, it is also likely that individuals, the peer consulting community and the wider organisation will gain an improved understanding of the nature of online peer consulting, therefore evolving the online peer consulting concept.

\subsubsection{Altering the plan for sessions}

The experiences gained through informal online peer consulting lead to restructuring or altering the plan for online peer consulting sessions. Assessing the outcomes of this period will highlight what worked and what didn't work for the community of 
peers, and reveal their preferred ways of interacting online. Systems statistics, observations, and feedback from the community are all valuable inputs into this assessment.

The plan for sessions developed at the Structuring stage can be though of as a blueprint for action, and as more is learned about the nature of the online peer consulting concept and the peer consulting community, this blueprint much be open to change. As the domain of a community of practice evolves, so must the sessions of a peer consulting community (McDermott \& Snyder, 2002).

Changes are made to increase the likelihood that formal peer consulting is more successful, and delivers additional value to the peer consulting community and the organisation. Changes may include:

- Timeframes

- Communication channel/mode

- Consulting issues and topics

- Frequency and length of sessions, and suchlike.

Assessing the outcomes of informal peer consulting and amending the plan for sessions will improve understanding of the realities of online peer consulting. As described in the literature review, the majority of organisations in NZ, and the organisations that took part in this study, are SMEs and operate in situations in which time, finances and expertise are in short supply. Therefore one of the influencing elements considered here is the support needed by an available to the peer consulting community (Riverin \& Stacey, 2008).

An amended plan for the series of online peer consulting sessions is the key output of this activity, which is used as the basis for action at the Engaging stage, and used for the next activity of the Experimenting stage, where the supporting online systems are reconfigured. 


\subsubsection{Reconfiguring systems}

As the plan for the series of sessions evolves, the set up of the supporting online tools is re-examined to ensure that they can still adequately support peer consulting activity. Information on the performance of the system and/or particular tools gained during informal peer consulting can also be considered at this point. I have termed this activity reconfiguring systems as it is likely that small changes to systems organisation will be needed, rather than a complete replacement of the selected system.

For example, changes may need to be made to the login process, the general availability of the system, the accessibility of tools, the organisation of functions within the user interface etc. Assessing, and if necessary amending, such aspects of systems configuration is important due to the impact that they can have on participation in the peer consulting community. Riverin and Stacey (2008) found that frustrations brought about by technical difficulties were a key reason that some people left a community. Reconfiguring systems to reflect changes in the plan for sessions and to address any problems community members faced during informal peer consulting should be carefully considered before the Experimenting stage is concluded.

\subsubsection{Mobilising point}

The activities of the Experimenting stage are characterised by improving the preparedness of peer consulting community members to take part in formal peer consulting sessions. A particular organisation and peer consulting community may undertake several iterations of informal consulting and changes to the session plan and system may occur. By experiencing online peer consulting, and being armed with a revised plan and online tools that can better support their peer consulting activities, the community is ready to leave the Experimenting stage and meet the challenges of Engaging in formal peer consulting. This improved preparedness is a Mobilising Point in the development of the peer consulting programme. 


\subsection{Engaging Stage}

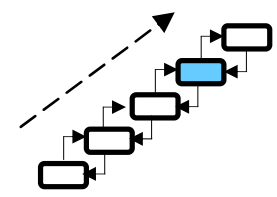

The activities undertaken in previous stages all prepare the organisation and peer consulting community for action at the Engaging stage. Here, ad hoc, unstructured peer consulting among smaller groups of peers still takes place, but the focus is now on more structured and formal peer consulting sessions; the online peer consulting concept and plan for sessions are actioned. As each session unfolds, the experiences of the peer consulting community are used to refine the process undertaken and the configuration of the supporting online system, thus formalising online peer consulting. Key actions taken at this stage are represented by three overarching activities:

- Participating in peer consulting sessions

- Formalising peer consulting sessions

- Refining systems set up

\subsubsection{Participating in peer consulting sessions}

The Engaging stage begins when the cycle of ad hoc systems use, and process and systems change of the Experimenting stage concludes. Although some peer consulting community members may still actively explore and test out the functions of the online tools, the majority of the community members are ready for more formal, scheduled online peer consulting sessions (McDermott \& Snyder, 2002). Although the sessions ideally have some common thread to promote continuity of experience, each session is also likely to have a slightly different focus, make use of different resources and/ or online tools, and follow an evolving process. A session may include some or all of:

- Accessing, reviewing and/or contributing to resources (text, audio, image, video)

- Commenting on session topics and/ or responding to session questions

- (asynchronously, synchronously, text, voice based)

- Engaging in open discussion

- Contributing to and/ or commenting on session outputs 
Engaging in an online peer consulting session for the first time is likely to present community members with some issues, such as the etiquette of online communication (Riverin \& Stacey 2008). Some community members will be less active contributors than others, especially during early sessions, and it is likely that some will remain lurkers throughout a series of sessions. As long as there is a healthy balance between lurkers and contributors, the peer consulting community can deliver value to its membership.

As the community moves through a series of online sessions, they will establish their own norms and these will become part of their shared history. Taking this idea further, engaging in multiple sessions enables a community to build a repository of individual and collective experience that endures after the sessions are concluded, strengthening their relationships and commitment to the peer consulting community. Also developed over time is a shared resource repository that forms an important part of the history of the community, and may be available to those who join the community in the future. Gongla and Rizzuto (2001) found that once a community becomes active in this manner, the wider organisation might experience value in terms of increased productivity of individuals, and the development of new procedures and practices.

The developing experience of the community leads to a deeper level of understanding of the peer consulting concept, and reveals any aspect of the peer consulting plan that is not supporting the community as intended.

\subsubsection{Formalising peer consulting sessions}

As the community engages in structured peer consulting, the aspects of the sessions that are and are not working - those that promote or inhibit engagement and learning - emerge. Formalising processes and other elements of a community of practice often takes place at early development stages, such as the Building stage of the Community Evolution model (Gongla and Rizzuto, 2001). However, within a peer 
consulting community processes and practices are likely to remain fluid until formal peer consulting sessions take place, and processes can be refined with the objective of providing a more meaningful and valuable peer consulting experience.

Participants draw on their experiences of peer consulting sessions to provide feedback on such aspects as session topics, structure, frequency, format, roles etc. Many such elements are types of coordinative activity as described by Akkerman et al. (2008) that occur before, during and after each session.

As a series of sessions unfolds, continual, iterative amendments are made, which leads to the sessions becoming increasingly formalised. This process requires an organisation to be in a state of change for an extended period of time, and their flexibility to promote and support change will be vital (Riverin \& Stacey 2008). As the nature of the sessions evolves, and the participants become more comfortable in the online environment, changes to the supporting online system are also made.

\subsubsection{Refining systems configuration}

Similar to the Structuring stage, as changes are made to the peer consulting sessions, subsequent changes need to be made to the supporting online tools to enable community engagement. Refining systems configuration is likely to be an iterative process, concurrent with formalising peer consulting sessions. The key changes made to the sessions are typically to the structure of each session, including the communication channel, resources and desired outputs, all of which are likely to bring about changes to the supporting online system.

Also considered here is the nature of community members' use of the system. At previous stages of the developmental process, use of online tools was deemed experimental. At the Engaging stage, the structured nature of sessions may promote more strategic and expanding use of such tools, as online peer consulting is incorporated into the usual work practices of participants (Chau \& Turner, 2002). If 
the nature of their use requires extensive changes (or additional features outside of the capacity of the selected system) another systems tool may be offered to complement or replace that originally chosen.

\subsubsection{Crystallising point}

Within the Engaging stage, the peer consulting community has participated in a series of online sessions. As these sessions took place, their experiences informed changes to the plan and structure of the sessions, and subsequently also to the online system. As peer consulting activities, session structure and the online tools come together to provide an optimal environment within which online peer consulting can take place, the peer consulting process and concept are crystallised, and the Crystallising Point has been reached. This sees the community reach the end of the period of Engaging, and begin the process of Embedding online peer consulting into the organisation.

\subsection{Embedding Stage}

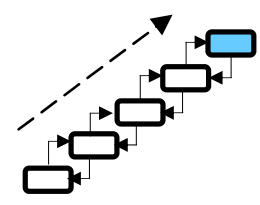

The final stage of development involves Embedding online peer consulting processes and practices into the organisation. Here, key organisational members work with the peer consulting community to integrate online peer consulting with existing professional development processes. I observed research participants undertaking many activities at the Embedding stage, which are organised into two interconnected groups:

- Cementing the online peer consulting concept

- Advancing technical infrastructure 


\subsubsection{Cementing the online peer consulting concept}

Once the peer consulting concept has been developed and actioned, the next concern is cementing the online peer consulting concept into the organisation. This involves developing the vision for what long-term online peer consulting will look like, including the processes, practices, outputs and value. In order to develop this vision, the outcomes of the previous four stages (Defining, Structuring, Experimenting and Engaging) are reviewed and specifically, the development of the series of online peer consulting sessions is assessed. The vision for online peer consulting should be assessed alongside other organisational practices and the organisational culture to ensure that it can be properly institutionalised. For example, an unstructured and fluid online peer consulting concept that includes ad hoc consulting and no predetermined supporting processes and systems is likely to be unsuccessful in a conservative, change-adverse organisation.

Cementing the online peer consulting concept also involves integrating it with existing professional development processes, whether they are in-house or external, formal or informal. This highlights one key difference between my model and other community development models, which is that the online peer consulting process and possibly also the peer consulting community endure. In Wenger's (1998) and McDermott's (2000) models, the community closes or 'dies' when, after a period of time it reaches the final stage of development. However, by integrating online peer consulting into a cohesive organisation-wide professional development programme there is the opportunity for the concept to continue to be developed, and for the peer consulting community to remain active. The community may be refreshed by new membership, consulting issues/topics and resources, similar to the Sustain stage of McDermott's model (2000).

\subsubsection{Advancing technical infrastructure}

The development of online peer consulting as a professional development activity may necessitate advancing the technical infrastructure of the organisation. Based on 
the nature of the use of supporting systems and tools at the Experimenting and Engaging stages, a picture of what is required for successful and continued online peer consulting can be developed. Similar to previous developmental stages, underlying technical infrastructure, this time organisation-wide, may need to be reconfigured, or additional tools introduced to the organisation in support of online peer consulting. Such activities should be assessed and planned for as part of this final stage of peer consulting transition, and undertaken prior to a full launch of online peer consulting in the organisation.

\subsubsection{Concretising point}

The learning gained in all stages of development has been brought together into a workable organisational practice of online peer consulting. When online peer consulting has been accepted and is firmly established as an organisational professional development activity, the Concretising Point has been reached and peer consulting transition is concluded.

\subsection{Chapter Summary}

In this chapter, I have presented the conceptual model developed in this study, as depicted in Figure 5, below. The five stages represent different groupings of participant actions, and the activities that they undertook as we collaboratively developed their online peer consulting concept and put it into action. While the particular research context of each organisation cannot be replicated, I believe that this conceptual model can act as a roadmap that guides organisations in developing their own peer consulting programme. 


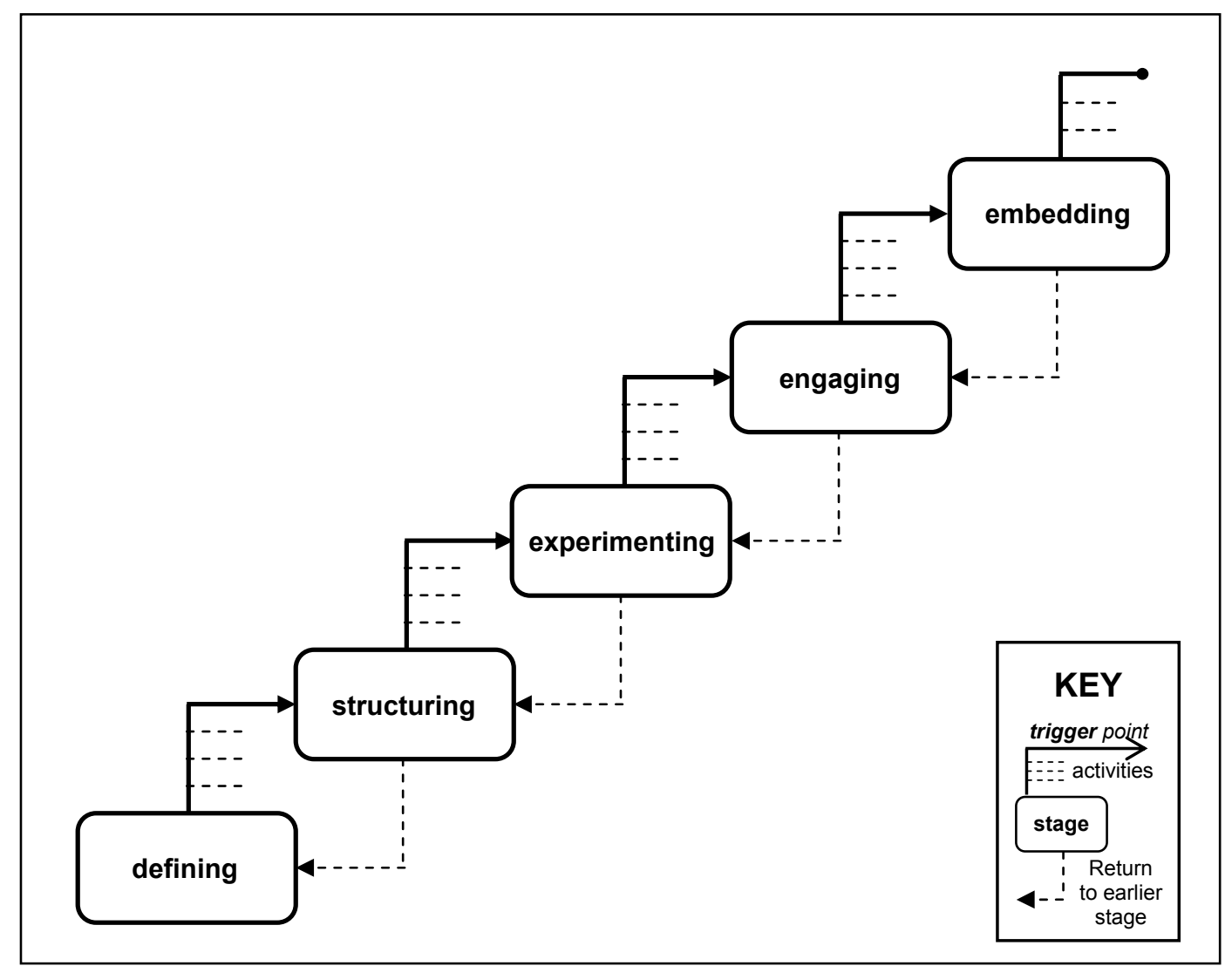

Figure 5: Model of Peer Consulting Transition

Some aspects of the model are deliberately brief, as they are discussed in other chapters of this thesis. Specifically, I elaborate on the use of technology for online peer consulting at the Engaging stage within Chapter 7. In addition, the activities introduced in this chapter are described in further detail in Chapters 5 and 6, as I describe the unique journey of each participant organisation along the staged Model of Peer Consulting Transition. 


\section{Peer Consulting Transition: The Story of Associated Counselling}

\subsection{Overview}

This chapter describes the pathway that Associated Counselling (AC) took when introducing online ICTs to support organisation-wide continued professional development. $\mathrm{AC}$ is a private sector counselling services organisation distributed across four regions of the South Island of NZ. A small team of staff deliver a specialised programme to youth clients, which includes a robust and proven counselling programme with a 'journey' component that involves group and individual therapy in the wilderness over a period of one week. At the time of this study, AC was a young organisation still in the start up phase of growth. As such, they were constrained by limited resources including finance, organisational infrastructure and specialist skills in other areas, such as technical skills ${ }^{6}$.

$\mathrm{AC}$ worked through many issues and activities when exploring their options for organisation-wide continued professional development. The Model of Peer

Consulting Transition (as introduced in Chapter 4) provides a frame within which I have placed the actions of $\mathrm{AC}$, each stage of development being distinguished by a number of key activities and a trigger point that combined to propel AC through the transition process to the Embedding stage. As some activities span across stages, or are repeated at various stages, they are presented here where I observed them to be most dominant.

As previously described, I developed the Model of Peer Consulting Transition by generalising from the unique experiences of the participant organisations, AC and CI. Therefore, AC's story of peer consulting transition, as presented in this chapter has

\footnotetext{
${ }^{6}$ See chapter 3 , section 3.4.1 for additional information about the nature of AC.
} 
some important differences from the model described in Chapter 4. Specifically, differences occur in:

- Titles of some stages

- Titles of some activities

- Order of some activities within stages

These are highlighted throughout the chapter and discussed further in the chapter summary. For ease of comparison I have organised this chapter in a similar way to Chapters 4, proceeding through five stages of the Model of Peer Consulting Transition before finally drawing several conclusions and diagrammatically presenting AC's overall journey (in Figure 8).

\subsection{Scoping at $\mathrm{AC}$}

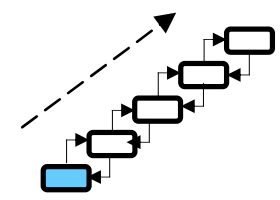

This is the first stage of peer consulting transition at AC, which involved assessing the current state of peer consulting and technology support in order to begin to develop their online peer consulting concept. Within the Scoping stage, the actions and experiences of participants are organised into the three categories of:

- Determining current peer consulting activities

- Discovering current ICT support

- Developing the online peer consulting concept

Each activity is discussed as a separate sub-section below, and together gives the overall picture of $\boldsymbol{S c o p i n g}$ the initial online peer consulting concept at AC.

\subsubsection{Determining current peer consulting activities}

Identifying the current peer consulting practices operating within AC revealed tension between supervision and consulting and highlighted the informal and fluid nature of the processes through which members of AC engage with peers. Exploring current practices also revealed several factors that inhibit peer consulting at AC, 
elaborated on below.

\section{Current peer consulting practices}

Within AC, many processes and organisational 'ways of doing things' were still being developed and instituted at the time of this study. One element of professional practice that was being assessed was the nature of supervision of and amongst peers. Members of $\mathrm{AC}$ have a more formal supervision contract as a part of their practicing requirement. This type of supervision takes place with a person who is external to $\mathrm{AC}$, and through my observation I deemed peer supervision more aligned with the concept of mentoring as described in the literature review of Chapter 2. Thus, peer supervision in terms of the supervision contract does not fall within the realm of peer consulting, and has been excluded from this study.

However, when exploring current peer consulting activities, key members of AC revealed some confusion between the use of the terms supervision and consulting, and when it was most appropriate to use each. P8 found that supervision did not adequately define many professional development activities of staff:

"Supervision is probably not quite the right word. You become more informed about your practice maybe by [discussing] it, and you reflect on your practice. I think that's probably a better description really. You reflect on your practice as you talk it out. But, to me that is not supervision. That is very much informal. That's not defined by any formalised kind of thing such as a supervision contract." (P8)

As the goal of this project is to complement current ways of consulting a peer, not to replace them, the terminology that participants were already comfortable in using has been applied, and the terms peer supervision and peer consulting used interchangeably when:

- there was a relationship of an equal nature among peers, and

- it was a helping relationship, and

- issues of practice were discussed, and

- parties to the discussions intended to personalise key aspects of the conversations to influence their counselling practice 
In the absence of these factors, participant actions fell within the more formal peer supervision process, as distinct from peer consulting.

At the time of the Scoping stage, peer consulting at AC consisted of loosely structured sessions that focussed on issues arising from casework. As up to four counsellors work within one regional office, these sessions typically took place faceto-face between co-located colleagues, although the regularity of sessions varied:

"It was our standard kind of once a week, or once every two weeks supervision session that we hold in [this region] because we've got so many people just within this one office." (P4)

Although several counsellors may be based within one regional office, work pressures meant that peer consulting was typically undertaken on a one-to-one basis, with few opportunities for a group or collective approach to be taken. Although oneto-one consulting and mentoring are recognised as valuable and legitimate forms of workplace learning (Stead, 2005), it is through peer learning in groups that professional practitioners effectively exchange ideas and leverage their existing experiences (Holbeche, 1996; Eisen, 2001).

Members of AC did state, however, that on occasion it is necessary to contact a colleague from another region, for reasons including:

- The presenting of an issue which is psychotherapeutic in nature, as there are few psychotherapists within AC

- Needing a fresh or varied perspective on a particular practice area

- When an urgent issue arises and no regional colleagues are available to consult

Counsellors from different regions typically attempted to engage in peer consultation via telephone call, and often after normal working hours. Although this was deemed necessary and colleagues were willing to be available for consultation, this situation was generally not preferred: 
"Usually that was a cell phone call. So what tended to happen was that the phone calls tended to happen late in the evening, which brought about issues about some of that boundary stuff about ringing people in their down time rather than in their work time." (P2)

$\mathrm{AC}$ recognises that peer consulting is an important organisational process, centred on the sharing of knowledge and the development and support of relationships. Consultation with peers is seen to be part of a wider professional development structure that $\mathrm{AC}$ is working towards developing and formalising.

When seeking advice from a peer, counsellors usually knew who had the expertise needed to assist them in improving their understanding of a presenting issue. This is largely because of the small size of the organisation and low staff numbers, and the strength of the relationships among peers. These connections, or networks are the means through which processes of knowledge search and knowledge transfer occur (Smith \& McKeen, 2003a) within learning activities.

The typical duration of a peer consulting event at $\mathrm{AC}$ varied, depending on the situation at hand and the level of urgency surrounding the issue. Additionally, the duration of a consultation was, at times, influenced by other events within the organisation and ranged from a quick phone call, to a longer discussion lasting one hour, up to an ongoing discussion via email and/or through a series of telephone calls. Issues such as the duration and urgency of a communication event fall into the category of contextual or situational factors, and influence the type of media selected (Watson-Manheim \& Belanger, 2007). The frequency with which peers engaged also varied, although participants reported that being consulted on an issue was a regular occurrence. The degree of regularity varied from multiple times per day for a more senior member of the organisation, to daily or weekly for others. The frequency of a consultation depended on who was involved, the nature of the issue, the time urgency involved (minimal through to high) and the other events going on within the organisation at the time, often termed institutional factors, that not only affect the 
frequency of communication, but the type(s) of media chosen to support communication (Watson-Manheim \& Belanger, 2007).

While most peer consulting took place between colleagues of a particular office, AC also holds twice yearly face-to-face meetings where organisational and practice related issues are discussed by all staff. However important it is for staff to meet face-to-face, these gatherings required significant investment:

"Sometimes there aren't huge amounts of material and we are transporting people down from [Region 1] to [Region 2], from [Region 3] to [Region 2], from [Region 4] to [Region 2] and that's adding up to well over a thousand dollars. And if we used some thing like [ICT] it may be considerably cheaper, in terms of travel time and the physical actual financial cost of it." (P4)

P4 also highlights the frustration experienced by $\mathrm{AC}$ staff at the lack of alternative professional development/ peer consulting methods available, and demonstrates an awareness of the potential role of ICT to support group consulting.

Exploring the ways that members of AC engaged in peer consulting reveals that while some peer consulting did occur, it did not occur as regularly as desired. This is largely due to several key difficulties in engaging with peers, as next described.

\section{Difficulties Engaging in Peer Consulting}

AC staff experienced a range of difficulties in engaging in peer consulting, brought about by factors such as the nature of their work, travel, the part time nature of some positions and the need to attend emergency client meetings. Participants also described more general difficulties, including:

- Connecting counsellors from within or across regional offices

- Responding to requests for help in a timely manner

- Being accessible and available to peers within and outside of usual working hours 
Due to the nature of AC work, meeting face-to-face with co-located colleagues was often problematic. P1 describes a typical experience:

"Because of the nature of the work, actually getting together a team of people within our workplace [is difficult] even though there's four of us here as counsellors and psychologists. To have two of us here is quite a feat let alone to have four of us" (P1)

and elaborates on the complexities of such situations:

"We've tried to do it here as a team. But um, you know, you schedule one and then on the Monday morning one person's got a child sick and another one has got an emergency meeting with a school or with some body and has had to run off, or um... Or you arrive and you've got an hour scheduled and one person is half an hour late and... it is really difficult to find those spaces" (P1)

One factor that disrupted regular peer consulting was the need for counsellors to travel to locations, sometimes remote, to counsel clients. A counsellor scheduled up to five clients and travelled for up to twelve hours in one day, being largely unavailable to peers during that period. Limited time resources and workloads are common barriers to engagement in professional development, Riverin and Stacey (2008) finding that actual or perceived lack of time was a large inhibitor to participation in voluntary online professional development initiatives.

$\mathrm{AC}$ holds values around peers supporting each other and fostering a strong sense of community. However, with few opportunities to meet face-to-face and difficulties in spanning the geographical distances between offices, peers often felt isolated. Golden, Viega and Dino (2008) discuss professional isolation as the desire to be socially connected in the workplace, and maintain a significant connection to networks of co-workers. A group of staff in one region of AC felt socially and professionally isolated from peers in another region, and a single staff member felt isolated from others, as $\mathrm{P} 2$ describes: 
"It's... again because of my geographical location and also the difficulty that I have with accessing professionals who are doing a similar type of work, or working with similar approaches." (P2)

Such isolation led to groups of counsellors within a particular office relying on the professional advice of their co-located peers, without the variety of perspectives that could be gained from consulting more widely. Consulting with colleagues from another regional office was described as a desired situation through which the pool of people to consult could be extended, thus opening up' the possibilities of peer consultation. Interestingly, P1 offers a positive view of the experienced professional isolation from colleagues in another region:

"One of the benefits too is that I don't have a day-today relationship with [peers from other offices], so the distance can actually provide some... it's kind of good to talk about some thing and then not have to see the person in the hallway further on in the day. It's quite good just to get in there, do some business and then get on with your day. And the isolation of it can actually be beneficial." (P1)

Striking a balance between peer support and developing and maintaining a sense of community within the organisation, and ensuring that there are opportunities for colleagues to obtain different viewpoints on their work is one professional development challenge AC faced.

In summary, peer consulting at AC typically took place informally, often in an ad hoc manner. As AC was still in its start up phase at the time of the Scoping stage, there existed few aspects of peer consulting that were formalised as organisational processes. Therefore, due to the unstructured nature of peer consulting, an accurate mapping of peer consulting as a distinct organisational process was not possible.

Peer consulting most often occurred on a one-to-one basis between the counsellors of a particular regional office. Little peer consulting took place between counsellors 
from different regions, resulting in localised knowledge at both an operational and managerial level.

In addition, the limited opportunities for face-to-face meetings mean that staff experienced social and professional isolation, some feeling a higher degree of isolation than others.

\subsubsection{Discovering current ICT support}

Various types of ICTs were used at AC to support peer consulting. As the nature and urgency of a presenting issue varied the communications channel also varied, from face-to-face contact, to telephone (landline and cellular phone) to email, and occasionally teleconference. Participants engaged in face-to-face consulting most often, followed by the use of the telephone and email for making initial contact with a peer, holding a discussion on an issue (typically synchronous via phone and asynchronous via email) and email was often used for exchanging information between peers. Counsellors who were more geographically isolated used cell phones more regularly to consult with peers. Similarly, Watson-Manheim \& Belanger (2007) found that people often preferred email and phone (or face-to-face) mediums for information gathering and simple knowledge exchange tasks.

The use of phone and email to support peer consulting caused difficulties. As counsellors were often in sessions with clients, had different work hours and needed to travel, they frequently engaged in 'telephone tag' with peers whereby they left several telephone messages for each other before finally making direct contact. This typically occurred across a three-day period, although on occasion lasted up to one week. Another difficulty of consulting via phone was the duration of a call, as "Phone calls tend to be a little bit unsatisfactory, in as far as that often they are brief." (P2) Reasons for brief calls included the workloads of staff and the cost of calls, from both landlines and cellular phones. 
Members of AC experienced similar difficulties when engaging through email.

Additionally, some counsellors did not have individual email addresses at the time of this research as the organisations infrastructure was still to catch up with the expansion of staff numbers. This led to problems contacting some staff, and a delay in distributing important information that was at times needed to inform or resolve a particular consultation, thus emails were often followed up by phone calls. When presented with more urgent issues, AC sometimes used teleconferences. Due to scheduling difficulties and cost, teleconferences were rarely used.

Various ICT have the potential not only to enable communications and knowledge sharing, but to enable such exchanges to take place in a timely manner and allow continual feedback and interaction (Garcia, 1993) that may overcome some of the frustrations $\mathrm{AC}$ experienced regarding the duration of a peer consulting exchange.

Assessing the technology used at AC revealed that peer consulting was not supported by Internet or intranet technologies, excluding the limited role of email described above. This finding was an important aspect of the next Scoping stage activity, developing the initial online peer consulting concept.

\subsubsection{Developing the online peer consulting concept}

AC developed their initial online peer consulting concept through scoping current peer consulting activities, exploring the nature of available ICT support and most importantly through generating a document outlining the nature of the project, trialling online peer consulting. This project outline briefly described the nature of the project, including the problem situation and its importance, the likely actions intended to relieve this situation, the duration of the project and the expected requirements and roles of $\mathrm{AC}$ staff and myself, the researcher (see Appendix C).

In early February (2005) a member of AC presented this project scoping document at a management team meeting, and several main aspects of the intended project were 
discussed. After an initial positive reaction, some elements of the scope of the project were amended, to improve data collection opportunities and clarify the privacy and security of organisational data. Once the scope of the project was agreed I met the management team and other staff at an organisation meeting (15th March, 2005) at which time we discussed the possibilities of the online peer consulting project. (See Chapter 3 for a description of an initial client/researcher meeting).

AC staff shared their experiences, and especially challenges, in engaging with peers. Specifically, they highlighted issues surrounding the low bandwidth connection at some regional offices, the reliability of hardware, and the difficulties scheduling group consulting. I discuss such issues in more detail in the Shaping stage, below. Through this discussion they agreed that peer consulting was an important organisational issue to pursue, and in further discussion they expressed interest in exploring the possibilities of using online technologies to support peer consulting.

From the Scoping stage activities, it was clear that value could be gained from online peer consulting at $\mathrm{AC}$, and they were enthusiastic about participating in the peer consulting project. From my observations and discussions with members of AC I determined that their initial online peer consulting concept should be centred on ideals of:

- Timeliness of engagements so that outcomes could inform practice

- Inclusiveness, involving all AC regional offices and staff

- Reciprocity, with staff giving and taking advice as equally as practicable

- Flexibility of format, scheduling and recording of engagements

- Simplicity in accessing and using supporting online technologies

\subsubsection{Scoping stage summary}

$\mathrm{AC}$ initiated their journey of developing an online peer consulting programme by exploring the ways that staff engaged in peer consulting within and across regional offices, and unearthing some of the main challenges faced. Peer consulting most often occurred face-to-face within offices, in an informal manner. Although a 
seemingly simple act, this process was, at times, hindered by elements such as the often unpredictable nature of AC's work and the distributed nature of the organisation.

Members of AC consulted peers from other offices, although they experienced several key issues that resulted in inter-office consulting being an occasional rather than usual event. Their experiences revealed at the Scoping stage showed that there was much to gain from more strategic use of supporting technologies.

With the knowledge gained during the above activities, $\mathrm{AC}$ began to sketch out their online peer consulting concept, pivotal elements of which included inclusiveness, reciprocity and flexibility. Exploring the nature and potential of online peer consulting at AC sparked the interest of management staff, and they were positive about furthering the concept. AC had, therefore, reached the Galvanising Point, at which time they ceased to asses current peer consulting practices and were propelled to the Shaping stage of peer consulting transition, where they turned to structuring their peer consulting programme.

\subsection{Shaping at $\mathrm{AC}$}

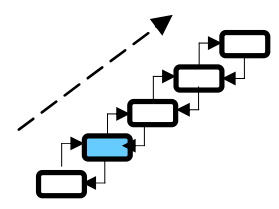

In the Shaping stage AC began to form their online peer consulting process, by applying the insights gained at the Scoping stage. More specifically, AC's potential process started to emerge as they more deeply considered the online peer consulting concept, while also assessing the technological tools that could best support the process. I have created two categories to describe the observed actions and experiences of members of $\mathrm{AC}$, namely:

- Honing the online peer consulting concept

- Selecting and setting up systems

These are discussed in detail within the remainder of the Shaping stage description. 


\subsubsection{Honing the online peer consulting concept}

The Scoping stage analysis revealed that there was no entrenched peer consulting process at $\mathrm{AC}$, due in part to the organisation being in the start-up phase. $\mathrm{AC}$ was first concerned with shaping their online peer consulting concept, rather than a distinct process. Many facets of their concept were discussed at the Shaping stage, most importantly some of the more practical and operational considerations and issues faced.

AC held values around peer support and worked to foster a strong sense of community. Members of the management team highlighted online peer consulting as a way to achieve this, describing online peer consulting as "another tool for supporting each other." (P4)

When considering current opportunities for peer consulting, participants also recognised that opportunities to reflect on their practice was an important part of their professional development, which they needed to regularly participate in:

"Key things for me is really the professional supervision and just being able to provide feedback to colleagues. But also to hear their ideas and share in different approaches. So, part of it is personal development, reflecting on their practice and also mine." (P2)

Although the nature of the work itself meant that they could work quite independently from one another, AC recognised and reiterated the need to shift from an individual to a group or collective approach to peer consulting, in order to gain a “different level of support" (P1). They identified benefits of gaining collective viewpoints on counselling issues, including saving time and obtaining additional insights on issues through group discussion: 
"I have a series of individual conversations, with different people, instead of a collective conversation that everybody is part of. And sometimes there are real advantages in having the collective situation." (P4)

However, AC did not have an efficient or effective way of undertaking regular group consulting within or across offices, and could have further benefited from combined approaches to issues and joint development of areas of their practice (Eisen, 2001).

Considering the current level of peer consulting within $\mathrm{AC}$ as a precursor to developing the online peer consulting concept highlighted several organisational aspects that were likely to affect the nature of online peer consulting:

- The flexible nature of AC

- The desire for synchronous consulting

- A focus on case-based issues

- Isolation experienced by staff

These aspects were taken into account when honing the online peer consulting concept at the Shaping stage.

\section{Flexible nature of $A C$}

Ingrained into the culture of $\mathrm{AC}$ is a willingness to work flexibly with colleagues and clients. As in many SMEs, the absence of many institutionalised formal structures enabled this flexibility in AC's operational aspects (Massey, 2004). It is necessary for them to be able to adapt to different working environments and situations, while remaining responsive to the needs of their clients:

"One of the key things about what we do is to be able to be flexible where we do a set of work with a young person, and then some times we do need to sort of extend the parameters of how we work." (P2)

This notion of 'extending work parameters' aligns with the concept of online peer consulting. Here, the goal is to compliment current peer consulting activities with an online peer consulting programme - extending the parameters of organisational 
professional development at AC. Key participants viewed online peer consulting as an extension of their current professional development arrangements, and described it as being "within normal supervision arrangements, but basically expanding the people that I can do it with." (P4) This notion of online peer consulting complimenting and extending professional development at $\mathrm{AC}$, and broadening the consulting group is in keeping with reported outcomes of the introduction of online professional development (Green \& Cifuentes, 2008). As this extension and expansion occurs, the responsive and flexible nature of the $\mathrm{AC}$ will need to be reflected in the flexible nature of the online peer consulting programme developed.

\section{Desire for synchronous consulting}

The core of AC's work was conducted in a face-to-face manner. Similarly, most peer consulting also took place face-to-face. When honing the online peer consulting concept $\mathrm{AC}$ was mindful of carrying over particular elements of such face-to-face interactions to the online space, to promote a higher level of participation in and success of their online peer consulting programme. Hildreth and Kimble (2005) found that when aspects of co-located community of practice were preserved in the online environment, the community was more likely to sustain and grow. In a similar way, transferring aspects of AC's current peer consulting practice online may promote the engagement of the community of learners.

Specifically, members of AC highlighted the real time (synchronous), and voice based aspects of peer consulting as important to transition from the face-to-face to online environment. Many reasons for taking a synchronous voice-based approach emerged from my discussions with AC. Most importantly, they described the immediacy offered by synchronous communications, allowing for clarifications of points made and resolutions of issues within one consulting session (Weick, 1979). $\mathrm{AC}$ also highlighted that voice-based (audio) consulting, as opposed to text-based consulting, offered a 'richer' communication, with a wider symbol set available to the consulting group. This is supported by the theory of media richness (also called the theory of information richness), which argues that voice-based communication typically allows a higher degree of social presence to be conveyed than text-based 
mediums (Weick, 1979; Daft \& Lengel (1986). Higher social presence and richer media would support AC in exchanging more information and potentially also improve their understanding reached within the timeframe of a peer consulting session.

\section{Focus on case-based consulting}

Similar to the desire to continue synchronous consulting, the case-based aspects of peer consulting were also identified a key element to transition to the online environment. The work of AC centres on client cases, and the need to consult with peers most often arises from issues faced when preparing to work and working with clients. Issues may relate to specific actions (of AC staff and/ or clients), processes, strategies, legalities and ethical concerns. One participant comment typifies this:

"I was certainly hoping to gain some increased learning myself by discussing other people's cases. And also to hopefully assist other colleagues on their cases and issues that might be coming up with at the moment." (P3)

Members of AC described group consulting as an effective way to stay informed about the cases, issues, strategies and insights of colleagues, as well as seek assistance with their own cases. They viewed the introduction of online tools to support peer consulting as a key enabling factor in providing staff with the opportunity to gain a group perspective on case-based issues.

\section{Isolation within AC}

Gaining colleagues' perspectives on case based issues was most important for the members of $\mathrm{AC}$ who were isolated from others. AC discussed the notion of professional isolation in more depth at the Shaping stage, describing the breaking down of isolation in terms of an organisational value:

"...to make sure that any one staff member is never isolated within our operation. That there's always a situation where staff members can access the thoughts and opinions and knowledge and experience of the others so that clients receive a safe and effective service." (P4) 
Building on this, another form of isolation was experienced by those in AC that practice a particular type of counselling, or have a particular area of expertise. Golden, Viega and Dino (2008) refer to social learning theories when discussing this issue, stating that organisational members are "at a disadvantage, and are forced, instead, to carry out work activities with limited insights, information, and feedback" (p. 1413). For example, there are two full time counsellors and one part time counsellor within $\mathrm{AC}$ who are also psychotherapists and are split between two regional offices. Issues have arisen that pertain to these counsellors that are distinct from other more general case-based issues, and psychotherapists have at times experienced difficulties in contacting a peer with the necessary expertise. One affected member of AC commented on the potential of online peer consulting in addressing this form of isolation:

"The big advantage is... I am a psychologist, [P9] is a psychologist. There are no other psychologists aside from [P8], who works point two down in the [other regional] office. And it means that if there are particular issues pertaining to us that are separate to the others, then we can just simply get online and have a conversation" (P4)

In summary, the general online peer consulting concept previously formed was developed further at the Shaping stage, becoming more relevant to the needs of AC. Specifically, it was necessary that:

- online peer consulting be consistent with $\mathrm{AC}$ values

- the online peer consulting programme reflect the flexibility of $\mathrm{AC}$

- online sessions focus on case-based issues and occur in a real-time, voicebased environment.

Identifying and discussing such aspects of online peer consulting at $\mathrm{AC}$ led to the honing of the overall concept. In response, no formal online peer consulting programme was developed at the Shaping stage, this occurred much later in the process, at the Familiarising stage (section 5.3, below).

After the scoping and subsequent honing of the online peer consulting concept, AC had a set of key elements against which to assess available online tools. Therefore, 
they moved on to consider which tools were most appropriate to support the concept developed thus far, as next described.

\subsubsection{Selecting and setting up systems}

Central to Shaping an online peer consulting process is making an assessment of the technology tools best able to support peer engagement. At AC, selecting and setting up systems comprised two distinct sub-activities:

- Assessing and selecting systems

- Setting up chosen systems

AC's journey through each activity is described below.

\section{Selecting systems}

As determined at the Scoping stage, peer consulting at AC was unstructured and not yet formalised at the time of this research. There was, therefore, no clearly defined peer consulting process that I could thoroughly assess and apply a particular theory to guide systems selection. Key members of AC and I collaborated to assess and select systems to support online peer consulting, guided by several media selection and online learning theories, as well as aspects of the unique organisational situation of AC.

Firstly, we considered the nature and types of issues likely to be discussed by AC. Members of AC described a wide range of case-based client issues on which they need to consult a peer. In addition to issues highlighted at the Scoping stage, issues faced include: 
- Ethical issues

At times, counsellors are faced with issues of an ethical nature. For example, the parents of a youth who is receiving counselling may want to know details of what was discussed in counselling sessions. An AC counsellor must deal with the client, the parents and often also the involved school in an appropriate way. As each client and case is unique, a counsellor often needs the advice of peers on the legalities of such situations as well as ideas on the best way forward for all concerned parties.

- Difficulties engaging with a client

Younger clients some times have an attitude or behaviour that makes it difficult for progress to be achieved. When a client is fully engaged with the counselling process, counsellors might seek additional ideas and advice from peers around strategies that might be more effective in engaging with the client.

- Journey issues

At a certain stage within the counselling process, a group of counsellors will take a group of youths on a wilderness trek for around two weeks, called a Journey. In addition to the normal preparations for a journey there are often other issues that arise that must be discussed by the group of counsellors, such as rules about smoking on journeys, dealing with aggression etc.

As AC discussed such scenarios, I formed a picture of some common themes:

- Involvement of multiple persons in each situation, from AC counsellors to clients to other involved parties such as school staff and counsellors

- Need to travel to counsel clients, or travel for other aspects of counselling work

- Some degree of urgency in resolving the situation or at least improving each parties understanding of the situation

- Geographical distance that separates peers

- Sensitive nature of the information involved in peer supervision situations

These factors combine to present problem situations involving a relatively high level of complexity. In addition, most situations faced by participants are also highly equivocal in that each party involved in a situation has access to different resources, 
including information and experiences (Lengel \& Daft, 1988). This results in the possibility of different interpretations of the presenting problem or issue and associated information. A theoretical assessment of key elements of these situations and the types of media that can support peers consulting on case-based issues was next undertaken.

\section{Theoretical considerations}

At AC, no formal peer consulting process existed at the time of this research.

However, I was able to identify and assess the communications processes within peer consulting exchanges, such as the situations mentioned above. To guide this assessment, I selected and applied Media Synchronicity Theory ${ }^{7}$. (MST) (Dennis \& Valacich, 1999; Dennis, Fuller \& Valacich, 2008). Media synchronicity refers to the extent to which individuals within a larger group are able to work on the same activity at the same time, also termed a 'shared focus' (Dennis \& Valacich, 1999). This theory was developed based on the argument that the key to effective media use is the matching of media capabilities to the primary communication processes required to perform a particular group task. MST provides a framework to assess the media that can best support communications processes that are inherently within tasks, termed conveyance and convergence:

- Conveyance:

O definition: the dissemination of a diversity of information from many sources, that is previously unknown to participants

- goal: to disseminate and obtain as much relevant information as possible to aid in understanding the situation at hand

\section{- Convergence:}

○ definition: comparison process whereby individuals compare their ideas to those of others in a group to form a shared meaning of an information set

- goal: for a group to agree on the meaning (common understanding) of information in the context of the current situation. The focus is on understanding each individual's interpretation of the information, not the information itself

\footnotetext{
7 see Appendix D for a detailed explanation of MST.
} 
MST combines these processes with a set media characteristics, namely: symbol variety, immediacy of feedback, parallelism, reprocessability and rehearsability as defined (see Figure 6).

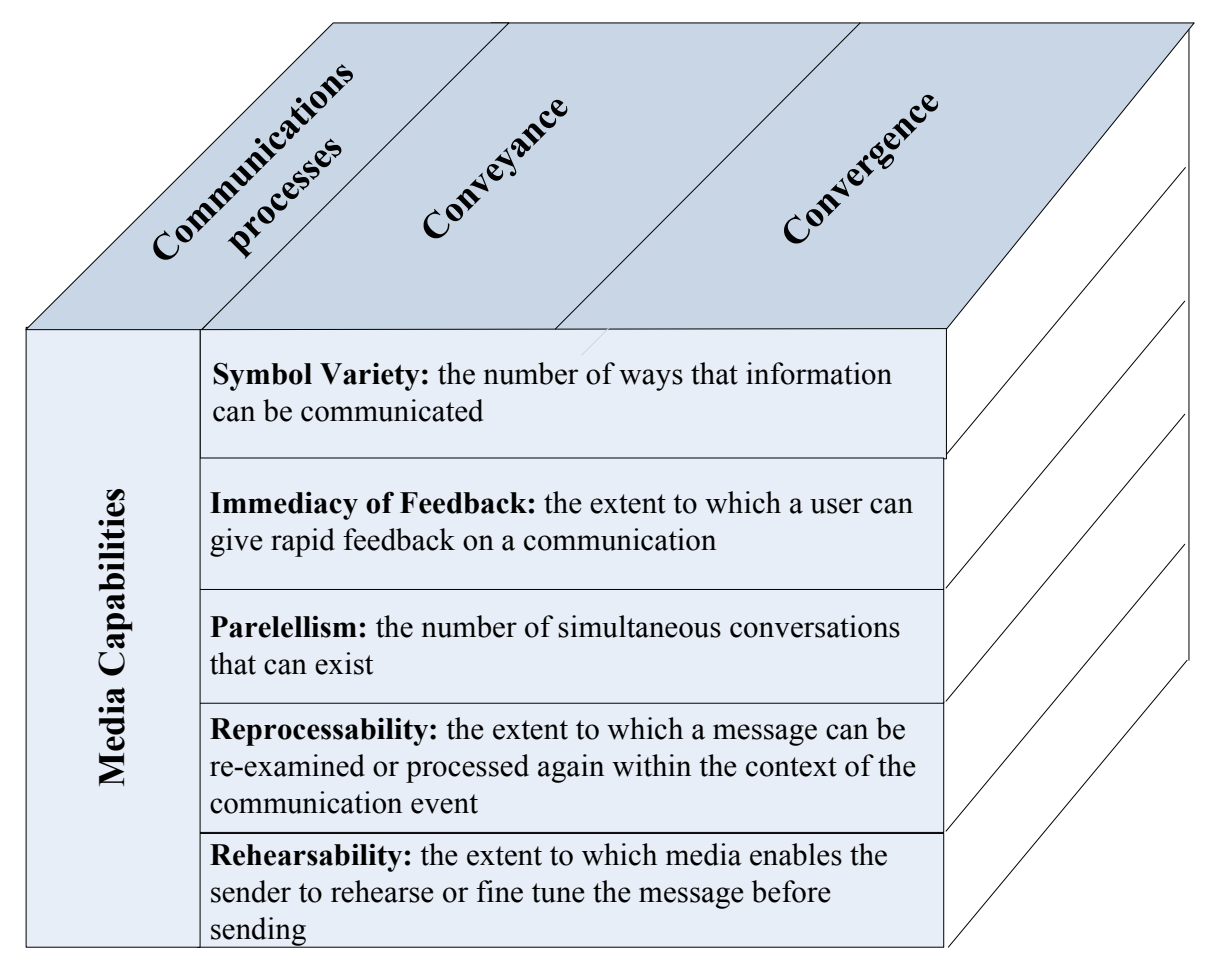

Figure 6: Elements of Media Synchronicity Theory (adapted from Dennis \& Valacich, 1999; Dennis et al., 2008)

In summary, the underlying premise of MST is that matching these five media characteristics to the communication processes of conveyance and convergence that are required for a particular task is the key to successful media use. Therefore, groups undertaking synchronous tasks (and to a lesser extent asynchronous tasks) need to select from a set of media that the group uses at different times when undertaking tasks, dependent on the prominent communications process. Furthering this point, Dennis \& Valacich found that most tasks require conveyance and convergence, and the media that is best matched to one process is often not ideal for the other. Therefore media switching was deemed most appropriate for groups that 
are performing synchronous tasks (Dennis \& Valacich, 1999). Media switching refers to "the act of changing media in the course of a conversation" and is reported to be increasing as additional ICT tools are available (Grandhi et al., 2003; p. 2142).

I applied MST to specify the ICT choices most appropriate in supporting peer consulting at $\mathrm{AC}$. When the need to consult a peer arises, it is reasonable to deduce that members of $\mathrm{AC}$ were either without essential knowledge or information, or held partial information. Also, at different times within the consultation process people may not have the knowledge or information that they require to continue a consultation or come to an improved understanding of the presenting issue. Some information exchange, whether factual in nature, through dissemination of documents or images may occur before, during and/ or after a peer consulting session to ensure that parties to the session have the needed information. This matches with the process of conveyancing. If a consultation event is not adequately prepared for, it is less likely that the parties to the consultation will reach an improved understanding of the issues presented and therefore will not be able to make an appropriate choice and move forward in the situation that they face. Key to conveyancing is a communications environment that supports low immediacy of feedback and high parallelism, defined as low media synchronicity (Dennis \& Valacich, 2008).

When meeting to consult on a case-based issue, members of AC typically attempt to understand colleagues' assessments and interpretations of case information. Resolving or attempting to understand a problem situation such as the case-based issues listed above requires peers to agree on a shared meaning of the information regarding the presenting issue within a particular context, from which appropriate actions can then be based. The prominent communications process described here is convergence (Dennis \& Valacich, 2008). Convergence should occur in an environment that supports high immediacy of feedback and low parallelism, as improving understanding typically requires that parties assess and reassess their understanding of information and that only one conversation exists at a given time. High media synchronicity is therefore key to the communications process of convergence. 
The informal and unstructured peer consulting currently undertaken at AC includes a mix of conveyance and convergence. This matches with the proposition of Dennis \& Valacich that most communications events require conveyance and convergence, although one may be more or less prominent at a given time. As the media that is best matched to one process is often not ideal for the other, media switching is likely to be most effective to support peer consulting within the participant group, whereby parties to the consultation will adopt the media (ICT) tool most effective in supporting their discussion, and switch to another supporting tool as the nature of their discussion and corresponding needs change. Due to the likelihood of ongoing media switching, it was important to carefully select which functions of a supporting online system to use during different stages of online peer consulting at AC (WatsonManheim \& Belanger (2007). Taking this into account, theory suggests that some appropriate media choices that will allow for a mix of low and high synchronicity needed to support online peer consulting at AC include:

\section{Conveyance}

Conveyance requires low media synchronicity, specifically the media characteristics of low feedback and high parallelism and high reprocessability. Appropriate media choices for peer consulting at AC include:

1. Written and electronic mail

2. Asynchronous groupware (threaded discussions)

3. Synchronous groupware (online chat, text based)

For exchanging information at the early stages of peer consulting, simple email messages were deemed adequate. Synchronous and asynchronous groupware could also support information dissemination and/or exchange, if needed. Participants could identify the person(s) with the required expertise via an online staff directory, and contact them via email to assess their willingness and availability for consultation. 


\section{Convergence}

Convergence requires high media synchronicity, specifically high feedback and low parallelism in media characteristics. Appropriate media choices for peer consulting at AC include:

1. Face-to-face

2. Telephone

3. Tele/Video conference

4. Asynchronous groupware (threaded discussions)

5. Synchronous groupware (online chat, text and voice based)

While I acknowledge that many other multimedia and communication tools are capable of supporting online learning communities and potentially peer consulting (for example see Bernard et al., 2000), the broad categories of synchronous and asynchronous groupware are likely to encompass other media.

By applying MST to the typical peer consulting situations experienced by AC, I formed an initial view of media appropriate to support online peer consulting. Theory alone gives an incomplete picture of the peer consulting issues and needs of $\mathrm{AC}$, therefore, I also assessed these media suggestions against some key organisational criteria, as outlined below.

\section{Practical considerations}

In previous developmental stages and corresponding activities, AC developed a refined online peer consulting concept and 'vision' for what online peer consulting might look like. Through this, a number of factors emerged that are important to take into consideration when assessing and selecting supporting systems:

- The need to travel to counsel clients

- The importance of discussing case-based issues

- Their preference for real time, synchronous communication

- Their familiarity with voice-based communication

Such factors align closely with AC's current peer consulting situation and reinforce the need to transfer, where possible, current ways of consulting peers to the online 
space (as previously discussed). Additionally, AC faces some constraining factors that guide their selection of systems:

- Financial constraints

- The level of existing infrastructure development

- The technical knowledge and abilities of the participant group

- Researchers technical skills and knowledge

- Project time frames

$\mathrm{AC}$ was still in the start up phase at the time of the Shaping stage. Due to this, the level of infrastructure was another factor carefully considered. Specifically, issues surrounding the low bandwidth connection at some regional offices and the reliability of hardware were raised and discussed. Such issues are not only commonly faced by start up organisations, but have are also present in many NZ SMEs, as reported by Massey (2004). AC highlighted that the systems selected must be accessible through a dial up internet connection, and not require additional investment in software or hardware. In addition, AC intended to expand the organisation and start up a new regional office, so the system selected needed to easily accommodate such changes.

Munkvold (2002) determined that an organisation such as AC with few employees that are mobile and separated by distance needed to support 'any time, any place' collaboration and also provide universal access to the services offered by a technology, in this case online peer consulting. As previously discussed, theory suggested that appropriate media to support online peer consulting at AC could include the use of teleconferencing and online text based discussion boards and chat facilities. Complementing this, discussion and feedback from members of AC revealed that a successful online peer consulting programme needed to be structured around synchronous, voice based communications. Therefore, AC's existing and more traditional communication channels such as face-to-face and telephone interactions remained important elements of their peer consulting concept. In order to move towards online peer consulting, and compliment existing peer consulting channels, I assessed systems that support voice over internet protocol (VoIP) exchanges and allow synchronous online engagements. 
Based on the requirements determined by AC's organisational situation and suggested by MST, a number of systems were assessed and the Chatterbox system was selected as the primary system to support online peer consulting at $\mathrm{AC}$ (see Figure 7).

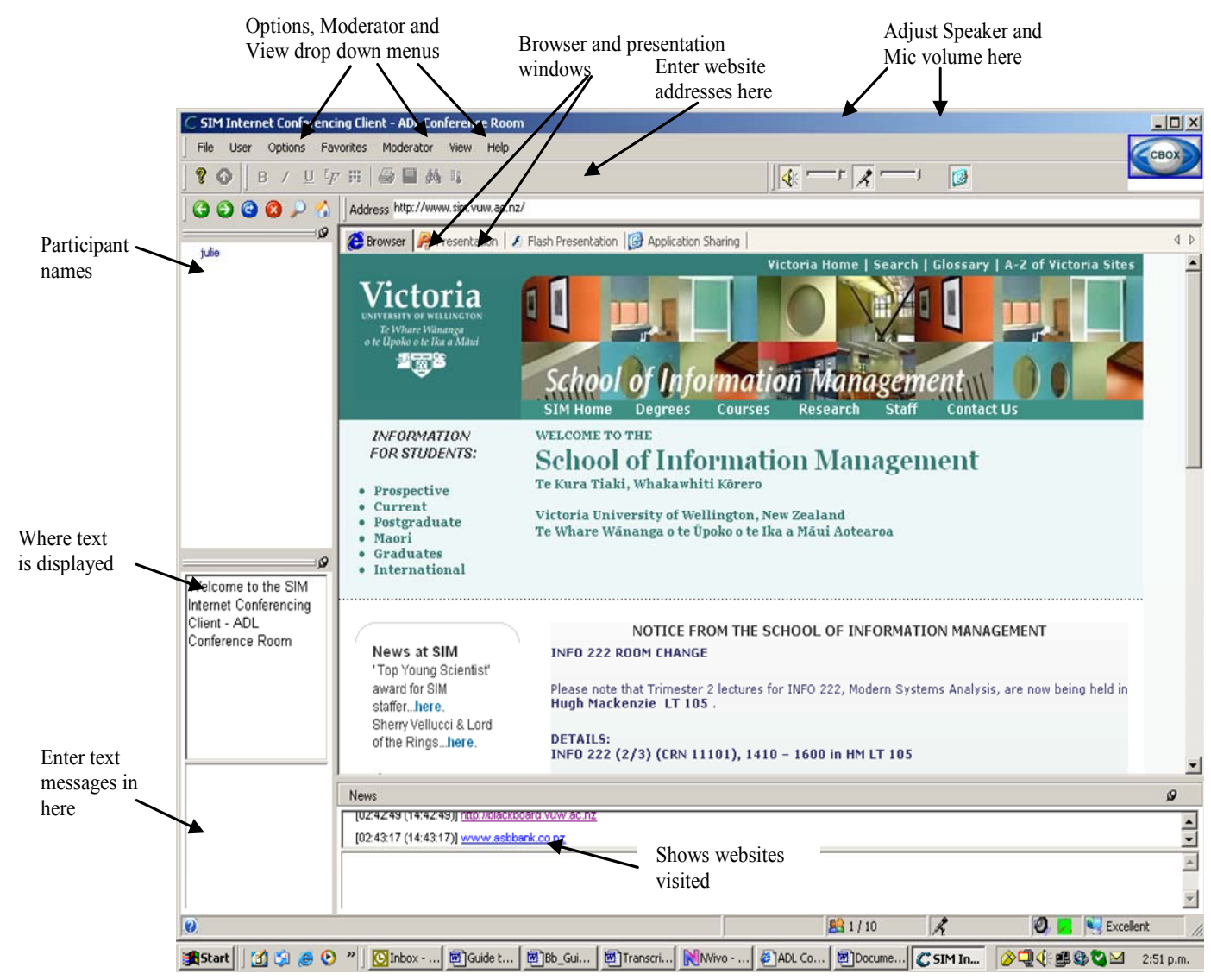

Figure 7: Example of the Chatterbox Window

Chatterbox is a Voice over Internet Protocol (VoIP) conferencing system that primarily supports synchronous, audio interactions and has additional functions including a text box for instant messages, and a web browsing window. Telephone and email adequately support the early stages of peer supervision, and Chatterbox was likely to be effective in supporting discussions within actual peer consulting sessions. As AC discussed the possible use of Chatterbox, many positive comments were shared: 
"My understanding of these Chatterbox's is so [P6] can get on Chatterbox from [Region 1] with maybe [P1] from [Region 2] and [P2] from [Region 4]. And they could get different feedback rather than taking their case issues to the same people all the time and maybe getting the same kind of feedback. To provide a variety of opinions." (P11)

In keeping with the suggestions of MST and the organisational situation described thus far, Blackboard was chosen as a complimentary system. As a learning management system, Blackboard was selected to support any asynchronous, text based communications between peers and the exchange and storage of documents needed for peer consulting sessions. After consultation, AC decided to proceed firstly with the Chatterbox system, and then introduce Blackboard further along in the series of peer consulting sessions.

In summary, consideration of AC's desire for group and voice-based consulting and other organisational issues combined with application of MST showed that peer consulting included both conveyance and convergence, and both communications needed to be supported. The selection of Chatterbox and Blackboard satisfied the previously listed criteria, as:

- Both systems were at no cost to AC (and required no additional technical infrastructure)

- Systems were available after a short set up period of approximately one month

- Systems were relatively simple to use

- Technical skills of participants would be adequate to use these systems after initial training

- Technical support needed was minimal, and could be provided by myself and my University

In addition to these two systems, we predicted that the use of email, conference calls and face-to-face meetings would continue. This discussion supports Dennis and Valacich's finding that media switching may be most appropriate. In this case media switching is demonstrated when participants select the media (communications channel) that is best matched to the nature of their needs. Here, groups of participants 
would choose between the set of different communications channels and functions of the systems media specified above, selecting the most appropriate media to support the prominent communications process of their peer consulting.

\section{Setting up systems}

Due to the nature of the chosen systems, setting them up ready for use in online peer consulting sessions was relatively simple, and occurred over a period of approximately two months. As online peer consulting was to be supported primarily by Chatterbox, this was the first system to be set up for AC. Setting up this conference room involved a number of key tasks, including naming the site, working with University technical staff to have user accounts created and activated, and systems testing. Members of AC were actively involved in naming the site and systems testing, which occurred at various times over a one month period.

While the Chatterbox familiarisation period was under way (as described in the Familiarising section, below) the Blackboard system was set up, which involved naming the site, creating and activating the user accounts, selecting and organising Blackboard templates, adding content to the various areas of the Blackboard organisation and systems testing. As the AC Blackboard site was developed members of AC viewed it and offered feedback, which then informed further changes.

\subsubsection{Shaping stage summary}

Building on outcomes of the Scoping stage outcomes, AC honed their online peer consulting concept by discussing in more detail aspects of the organisation and the ways in which staff preferred to engage with their work and each other. Furthering their understanding of the desire for synchronous consulting that focuses on casebased issues, the isolation experienced by many staff and the flexible nature of the organisation, enabled $\mathrm{AC}$ to ensure that their online peer consulting concept was consistent with organisational values and reflected accurately the needs and desires 
of staff. AC then applied this knowledge along with MST to the assessment and selection of supporting online tools, deciding on two complimentary systems of Chatterbox and Blackboard.

The Shaping stage activities of honing their online peer consulting concept and selecting and setting up supporting systems were an important step in preparing for formal online peer consulting sessions. Improved understandings and early preparations for sessions heightened AC's interest in and motivation for online peer consulting, and they reached the Rousing Point. AC then accelerated to the Familiarising stage, in which they put their honed concept and online system into action for initial, ad hoc online peer consulting.

\subsection{Familiarising at $\mathrm{AC}$}

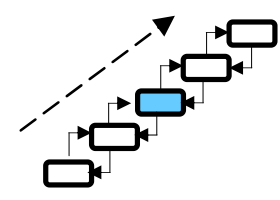

The Familiarising stage is where AC first put their organisational online peer consulting concept into practice. In keeping with the flexible, dynamic nature of the organisation AC moved into a period of learning about the online system through 'training in action', and experimented with the system through unstructured online peer consulting. Next, AC integrated the knowledge gained through developing their concept and selecting systems with their experiences of unstructured online peer consulting and developed a plan for a programme of structured online peer consulting sessions.

I have grouped activities in the Familiarising stage under the titles of training in action and planning online peer consulting sessions, as detailed below. 


\subsubsection{Training in action}

"I guess the most immediate thing was I wanted to come away with an understanding of what Chatterbox actually did, and how it could be useful for our staff." (P4)

The core purpose of training in action was for AC to progress from developing the concept to gaining direct experience of the online system and how it can be used to support peer consulting. This was accomplished through AC participating in both direct and indirect forms of training, the combination of which I have termed 'training in action'. I have distinguished this from training outlined in Chapters 4 and 6; AC did not prepare for or undertake any specific training sessions or predetermined training tasks.

Indirect training involved members of AC independently following through the steps of a written training guide as an introduction to the system. This guide included installing and logging on to the system and a brief introduction to using the basic systems features. As I was geographically separated from members of AC, and they were separated from each other, the written guides provided a way for each member of $\mathrm{AC}$ to receive training at their convenience without significantly disrupting their work schedule.

By receiving and working through the guides, $\mathrm{AC}$ found additional motivation to engage in the ad hoc peer consulting of direct training. AC participated in direct training and experimented with the system in small groups, while I provided assistance via phone, email and/ or the system itself. Chau and Turner (2002) term this type of technology use 'experimental', and found that it is most evident at early stages of technology adoption and diffusion, such as the Familiarising stage.

This early opportunity to trial the system enabled members of AC to become more comfortable with the online communications format in a relatively short period of time, as suggested by Riverin and Stacey (2008). In addition to this more general 
experience, $\mathrm{AC}$ also undertook direct training on particular functions of the online system. For example, peers supported each other in learning how to use the text facility of the system:

"We experimented a little bit with the text function after the consultation session. Just to familiarise people with it. For example, [P2] wasn't sure how to enter text and so we just went through that. He just entered in some text, pretty random sort of information. It wasn't actually used as part of the consultation process." (P9)

As participants had varying levels of experience using the system, such training occurred at different times during the Familiarising period. Ideally, all AC staff would have undertaken training in action and experimented with the system before the first formal online peer consulting session took place. However, this was not possible due to barriers including their schedules and priorities of their work and other events outside of AC. As a result, some members of AC attended their first formal session having limited (or no) experience of the system. As a result, P9 led training in action at the beginning of a consulting session:

\begin{abstract}
"This is the first session for some of the people doing Chatterbox. So we spent the first part of the session talking about how we might do it. That's everybody toing and fro-ing. But eventually... to be honest in fact I presented the case, just to kick it off really, to kick off the session so that there was not so much a model, but so that they could see it being done." (P9)
\end{abstract}

Such leading by example was an important aspect of training in action and the progression of the online peer consulting programme at AC. Hildreth et al. (2000) identified the facilitation of participation in online communities as one of the most difficult areas in the transition to the online environment, and the role adopted by P9 (and others) eased this transition for AC's community of learners. Effective facilitation and coordination of online peer consulting sessions was a critical factor in later stages.

Through training in action, AC further developed their capability to participate in online peer consulting and gained increased confidence in the online peer consulting 
concept. Although described here as a core activity of the Familiarising stage, training in action was ongoing and did also occur, although less frequently, within the formal online peer consulting sessions of the Engaging stage (see section 5.4, below). As individuals gained additional experience with the system and explored some of the extended functionality, such additional training in action took place.

\subsubsection{Planning online peer consulting sessions}

AC considered many factors when planning the series of online peer consulting sessions, including frequency, duration, group size, topics and ways to structure each session. These factors are divided into those that detail the overall nature of the series of sessions, and those that detail the structure of each individual session.

Firstly, the series of sessions was bounded to a three-month period. This timeframe allowed enough sessions to occur and took account of disruptive events, such as school holidays (that interrupted counselling sessions of younger clients) and organisational events that require staff to travel for around one week at a time. To allow for easier scheduling and greater participation sessions of around one hour duration were planned to take place fortnightly to begin with, then monthly at a regular day and time:

"If you are having a Chatterbox supervision session, being able schedule it during the day, and people being able to organise their diaries so that they can participate, I think that's another bonus about what we have really." (P2)

An important driver for introducing online peer consulting at $\mathrm{AC}$ was reducing staff isolation. In keeping with this, fortnightly sessions were designed to include around four staff, a mix of co-located and more geographically isolated peers. As previously described, the nature AC's work resulted in staff often working quite independently from one another, and therefore group consulting needed to be carefully considered to ensure a good fit was achieved between existing practices and online peer consulting. 
$\mathrm{AC}$ also took into consideration the likelihood of the organisation expanding to new regions, and opening additional offices. Therefore, it was vital to keep enough flexibility within the series of sessions to include new staff when needed. Weaver et al. (2008) posited that successful, institution-wide professional development was wide ranging, which included flexibility and transferability of timing of professional development, type of professional development and resources (p. 676). Another idea put forward was to include the online peer consulting programme as part of the induction of new staff, to encourage cross-organisational communication and engagement and promote the value of strong working relationships and sense of community at AC.

After determining the overall nature of sessions, $\mathrm{AC}$ assessed the structure of each particular session, identifying three options:

1. A facilitated discussion based on a topical article

2. A facilitated discussion on the structure of peer consulting at AC

3. A case-based discussion, using the 'Hewson Triangle'

The first option involves someone being tasked with posting an article on a practice or process-related topic or issue, and facilitating a more open discussion based on that material. Shared documents can act as 'collaboration catalysts' for discussion, encouraging group members to apply their knowledge (Hildreth et al., 2000; Kimble \& Hildreth, 2005). As participants saw more value in discussing current, case-based issues, this option was not pursued. The next option considers questions such as "how does peer consulting fit with other professional development activities?" and “does Coy 1 need a formal supervision/ consulting contract or process?" P2 described how such a discussion could begin to develop a model and some shared terminology around peer consultation within AC. Although this was not used as a topic for a specific peer consulting session, the nature of peer consulting, and online peer consulting at $\mathrm{AC}$ was discussed as part of several sessions, as described in the Engaging stage. 
The option that was deemed to potentially offer the most value is that of framing sessions around case-based discussions, following a clinical supervision framework developed by Hewson (2002):

"We attended a supervision seminar with Daphne Hewson last week and the 4 of us who attended all thought that some of her material would work well in the context of the online supervision." (P9)

Elaborating further, they anticipated that use of the Hewson model would enable a peer consulting group to gain a deeper understanding of a case and any associated issues raised. (see Appendix E) A group could move through the three sections of the model in about one hour, which is a similar time period to that of face-to-face sessions. A last important aspect of planning for the series of sessions was that AC recognised that one-hour case based sessions would be most like the current face-toface sessions, and perhaps allow for an easier transition from face-to-face to online peer consulting. The additional structure and focus that the Hewson Triangle could bring to sessions is likely to provide some consistency across the series of sessions and improve the depth and breadth of discussions, bringing further value to staff. Embedding structure into the sessions in this manner goes some way to formalising the collaborative practice of the sessions (Akkerman et al., 2008).

\subsubsection{Familiarising stage summary}

AC gained first hand experience of online peer consulting through training in action at the Familiarising stage. Although they experienced some minor technical, scheduling and other difficulties during this period, experimenting with the voice and text functions of the system helped $\mathrm{AC}$ to further develop their understanding of the possibilities for online peer consulting sessions. Training in action also raised the confidence of individual AC staff in their ability to navigate the Chatterbox system and participate in online peer consulting.

Drawing on the accumulated outputs of the Scoping and Shaping stages and their newly gathered experience of informal, ad hoc online peer consulting, AC developed 
an outline of a series of sessions to be actioned at the Engaging stage. Through this planning AC determined that online peer consulting would be most successful by holding up to six sessions over a three month period, where they discussed casebased issues within the organising framework of the Hewson Triangle.

$\mathrm{AC}$ further developed their capability to participate in online peer consulting, and had a plan for continued consulting at the Engaging stage. Reaching this level of preparedness to engage in formal online peer consulting motivated them to move forward from Familiarising activities, and thus, this is the Mobilising Point that promoted $\mathrm{AC}$ to the next developmental stage.

\subsection{Engaging at $\mathrm{AC}$}

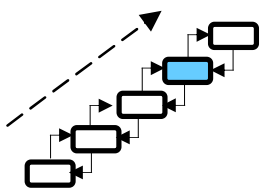

Soon after AC reached the Mobilising Point they put their online peer consulting concept into action and engaged in a series of formal sessions. Each session involved a different mix of peers and the presentation and discussion of a different case. As the sessions progressed, $\mathrm{AC}$ reflected on their experiences and incrementally formalised the peer consulting process, and subsequently reviewed their use of the supporting system. Their actions, as I observed them, are represented by the key activities of:

- Performing formal online peer consulting

- Reforming the online peer consulting sessions

- Reviewing systems use

Within this section, I elaborate on each activity and highlight key themes.

\subsubsection{Performing formal online peer consulting}

Most members of $\mathrm{AC}$ had participated in indirect and/or direct training-in-action at the Familiarising stage, and were ready to engage in formal online peer consulting 
sessions. Their experience of ad hoc online consulting acted to reinforce the desire for group consulting and gaining collective viewpoints on case-based issues. Indeed, $\mathrm{P} 3$ viewed this as a key motivator for the session:

"The need for the session was something that was planned beforehand about realising, as part of our work we need to have regular supervision and it is really beneficial to use a group process." (P3)

Additional motivators for participation included talking through and receiving ideas for a current case, hearing strategies for dealing with a particular issue, understanding others' approaches to a particular policy, and for one person, also trialling the online system.

\section{The nature of sessions}

AC carried out six sessions, held fortnightly over a period of approximately three months. Most staff attended multiple sessions, with three to five people present at each session. The typical process undertaken for the case-based description and discussion was for the 'case owner' or presenter to notify others and send through some case background via email prior o the session. P9 summarises the process followed within a session:

"What happened first was that I presented some of the details of the case and the background. I was pretty much following... Daphne Hewson's supervision model, the triangle. I started on the client side of the process and worked through some of those areas. And then worked around to stating what were some of my issues, why I was doing the case. And then once I'd done that I asked for comments, and part way through I stopped and asked if any one wanted to ask any questions. At that point people were happy that we just continued. And so I continued on with the presentation. Then there was a series of questions, to seek clarification from different people... And then they made some comments, some suggestions. And there was some dialogue. And once we'd done that for a while, I took my own notes at that point." (P9) 
As AC progressed through the series of sessions, they found the Hewson Triangle was effective in guiding them to focus on the pertinent case points, and valuable in allowing a deeper analysis of a case.

Within each session, two people usually presented cases of around 15 minutes duration each. The remainder of each session was made up of questions and discussion, as well as some reflection or debrief on the session itself. Due to the nature of the issues discussed and the communications format of the online system, they kept sessions to around one hour:

"We all felt that our session was long enough. That actually, you had to concentrate and listen quite hard. I guess that is similar to a normal supervision session. But much longer than an hour would be too much." (P9)

The structure and duration of each session was similar to that of a face-to-face session. The process followed what P4 described as "quite a similar model in terms of the presentation of the background, and then of highlighting the ideas and questions and issues that may need to be addressed" and then moving on to a period of "dialogue, questions, suggestions and responses" (P4).

Structuring each session a similar way provided some consistency and continuity across the series of sessions, and also continuity of experience for $\mathrm{AC}$ (Wenger, McDermott \& Snyder, 2002). Such continuity was vital in enabling AC to develop shared understandings on issues, a growing comfort and confidence with the sessions, and some sense of routine around reflecting on one session and preparing for the next, which provided what P2 termed "flow though" within the series of sessions. McDermott (2000) found this to shift a community from a group of people who share an interest, to one that develop a practice.

As described in the Familiarising stage, sessions were scheduled, occurring at a prearranged fortnightly day and time. Therefore, urgent issues or crisis situations were not brought to the sessions, and were dealt with through other channels. 
Although there was typically a low or sometimes moderate level of urgency in sessions, many examples of the advantages of gaining timely advice from colleagues through online peer consulting were given, for example:

"The case I did discuss was certainly an area where having some timely input was important. And so what that did, was that actually sped up the process somewhat. Whereas, had I not had that session I would have had to have probably had a session with someone else, and that would have delayed the process. So I guess I used this Chatterbox session to short circuit, or to shorten, rather than short circuit, to shorten the length of time to the peer consultation that I would have needed to have any way." (P9)

In summary, during a three month period $\mathrm{AC}$ undertook six online peer consulting sessions of around one hours duration each, through which they consulted on casebased issues. As these sessions unfolded, they reflected on the key outcomes experienced, described here as emergent issues.

\section{Emergent issues}

"Because of my geographical location and also the difficulty that I have with accessing professionals who are doing a similar type of work, or working with similar approaches, being able to have these supervision sessions I see as being vital... I'm also appreciative of the fact that the people in [other regions] and those who are in offices and have colleagues in the office have made the effort to include me... And the other part of it is also that ongoing relationship within AC and the sharing of ideas. I think that's the foundation really of the work that we do with clients, seeing as that we do need to actively find times to reflect on our work and the group supervision really is just a need to be preformed. And...I think that actually I find that Chatterbox actually means that it's quite an intense focussed period. I'm going to have to work quite hard and feel, like... afterwards probably I do need a bit of a breather after that before going off and moving into a counselling session because of the level of intensity that I'm working at." (P2)

Here, P2 highlights some of the key issues that emerged as AC experienced online peer consulting, specifically: 
- The intensity of sessions

- Breaking down professional isolation

- The importance of reflecting on work

\section{The intensity of sessions}

Within a face-to-face peer consulting session, $\mathrm{P} 4$ noted that interruptions from peers often break their train of thought, and at times detracted from the overall quality of communication. As Chatterbox structures dialogue, allowing only one person to speak at a time and forcing others to wait for a speaker to pass the right to speak to them, less interruption is possible. This allows the case presenter to speak their entire train of thought, which gives some additional clarity around the case and issues, P9 described the situation as being able to develop the case in the way that it was in their mind, allowing them to "speak more fluently and coherently about a case" (P9). This implies that low immediacy of feedback was a positive aspect of the online session, and the Chatterbox system was well matched to the particular communication task (Dennis \& Valacich, 1999). Resultantly, parties in a consulting group had greater focus on what they require from those they are consulting with, described as improved "intensity of focus" (P2).

This additional level of discipline that "forced them to listen" (P4) to the case presenter and other speakers took a while to get used to. However, after attending a number of sessions they found that they were more focused in on what the presenter was describing to them, and were subsequently able to offer more focussed responses and ask more considered questions:

"The more I used it the more I found it was actually really good that you couldn't interrupt. I think sometimes that there is tendency that I have that I jump in before people will finish. If I have a good idea, I like to share it. So, one of the things that I found is that it did really require some reasonably focused listening. I also found that I made notes as you went along the way. In some ways I think that there was the opportunity to do quite a bit of reflection before talking, before pushing the control button to be able to speak. So I found that it kind of slowed down the interaction but there is the real benefit for me in that... So one of the benefits for me was also around that we 
were really quite focussed on the task, on dealing with the case of supervision itself without having those wider social interactions take place. Because the other social cues, the face-to-face interactions weren't there." (P2)

Having a more focussed, and at times more intense discussion was also described by P3 as a better use of their time, as socialising at the beginning of a session and straying off topic were less likely in the online environment.

\section{Breaking down of professional isolation}

At the Scoping and Structuring stages, participants identified the lack of opportunities for face-to-face meetings and the resultant professional isolation felt by members of $\mathrm{AC}$ as a key reason for introducing online peer consulting. This was reflected on as $\mathrm{P} 1$ gained more experience of online peer consulting:

"I think that one of the problems that we have is that our ability to meet face-to-face is quite limited. So, it sort of provides a bridge in the interim, you know, like between our face-to-face meetings." (P1)

As AC progressed through the series of sessions, this bridge was further extended.

Those who were co-located prioritised the inclusion of one or more of their more isolated colleagues in their usual peer consulting sessions and often encouraged them to present a case at the beginning of a session, thus beginning to relieve the professional isolation faced. Many participants were mindful of this need, a typical statement being:

"The session that we had this morning is based around supervision, clinical supervision of counselling, available for all of our staff to come online. And mainly for those people who are working by themselves so that they can get peer supervision within our team. And there were three of us from the [Region 2] office, and [P2] from [Region 4]." (P11)

As previously discussed, a core value of AC is the strength of staff relationships and the sense of community within the organisation. This sense of community, termed 
the 'social climate' (Bernard et al., 2000; Saint-Onge and Wallace, 2003), becomes increasingly important as AC moves through the series of sessions, and is vital in enabling each isolated peer consulting session to become continued professional development (Conrad, 2005). The management team viewed offering increased opportunities for staff to engage with peers on practice-related issues as one way to improve the organisation-wide sense of community:

"When I set up this company I wanted us to have a strong sense of community. And I just see this as offering another piece of that sense of community." (P4)

As participants engaged online they experienced the potential of Chatterbox to support their professional relationships by enabling improved opportunities to engage with geographically disparate colleagues, and focussing interactions on clinical issues. This was identified as a distinct advantage of online peer consulting over face-to-face and phone interactions, as (P2) expressed:

"For me, I think that it's going to be really useful. Particularly connecting up those key people whose work I admire and who's thinking. Particularly, from the general relationships and doing that group work with them, is that it is a way of being able to keep it on a clinical counselling framework. Because most of the other time when we are meeting it's in a management area, discussions about management rather than around case issues. So for me, it's great actually, it's a really good tool." (P2)

Both those who were the sole AC staff member in a region and those temporarily isolated due to the absence of co-located colleagues conveyed positive impacts of having increased opportunities to engage with peers.

\section{The importance of reflecting on work}

Engaging in online peer consulting reinforced their need as professional practitioners to regularly reflect on their current practice. This was not only an outcome of the sessions, but also as a motivating factor in session participation. Webster-Wright (2009) notes that there are different interpretations of 'reflection' in the field of 
professional development, but also goes on to describe many studies, strategies and theories that highlight the critical importance of reflection in professional learning and professional development. The need for reflection was fulfilled when presenting a client case within a session, or being part of the audience, the consulting group listening and responding to the case presentation. The benefit gained when presenting a case and receiving advice and feedback from peers was anticipated. However, it was not expected that so much value would be received from being part of the responding group:

"I think even when you didn't have the chance to reflect on your own clients, the value of listening to other people talk about their clients and the ideas... because we all work with the same age group and the same issues. You could've turned [Chatterbox] on and not had a microphone and benefited greatly from just listening to that conversation. And I think for me that was really rewarding." (P1)

Most members of $\mathrm{AC}$ also discussed the individual reflection, termed "individual contemplation" by Webster-Wright (2009), that took place after each session. P2 found "there's actually a lot of reflection that goes on afterwards," and aspects of this post-session reflection became preparations for the next session. Discussion of such preparation highlighted the importance of scheduling a session and planning to reflect on client work, as opposed to only contacting a colleague in an emergency or urgent situation was another benefit of the online peer consulting programme discussed by participants:

"Normally when we phone each other it's very reactive, it's very fire fighting, you know, 'I'm really worried about this situation, what do we do?' Whereas this was an occasion where we were actually doing some thinking and planning for the client, using these guys as a reflecting team, the rest of the people who were involved" (P1)

P1 also raises another key aspect of reflection through online peer consulting; the opportunity to have a 'reflecting team' of colleagues to question a presenter, and offer their individual and collective knowledge and strategies on a clinical situation. Webster-Wright (2009) notes that critical reflection involves the challenging of 
assumptions, which is best achieved in a group situation. This experience was compared to reflecting team training that a number of participants had attended at University, and had few, if any, opportunities to emulate since. As previously discussed, the value of reflecting on work as a group was linked to helping with current issues and cases brought to sessions. Interestingly, reflecting on case work also acted to improve participants understanding of past cases, and prepare them for engaging with client cases that presented with similar situations and issues in the future. Overall, AC's experiences of online peer consulting show the importance they place on regularly reflecting on issues of practice, in a group environment.

A final key indicator of the importance of reflecting on work is the gradual formalising of the online peer consulting programme. At the outset of the programme, there existed little structure and formality around organisation-wide peer consulting at AC, excluding the staff face-to-face meetings described at the Scoping stage, which is typical of an organisation that is in a start up phase (Massey, 2004). As online peer consulting progressed, sessions became increasingly formalised, as discussed in the next sub-section.

\section{Key disruptions to the online peer consulting sessions}

During the Engaging stage, AC progressed through six online peer consulting sessions, and experienced some recurring barriers to participation that warrant mention $^{8}$. Many disruptions to online peer consulting experienced are typical of organisational life, such as staff taking leave for holidays, periodically travelling for work-related events and or being absent due to illness. In addition, the nature of their work resulted in a high client load and conflicts of appointments, which in turn sometimes led to a lack of clarity around who was available to participate in a particular session:

“...you can find out a week before that you've got a family group conference or a meeting with the police scheduled at nine o'clock on Monday morning and that would take precedence over the Chatterbox supervision." (P1)

\footnotetext{
${ }^{8}$ Inhibiting and supporting factors are discussed further as part of the Technology-in-Practice analysis of Chapter 7.
} 
When faced with schedule conflicts and staff absences, the flexible culture and nature of $\mathrm{AC}$ meant that they adapted, and adjusted each session to include those who were able to attend. In one instance, a member of AC joined a session part way through:

"[P11] actually came in part way through the consultation process. So, she really missed my presentation and discussion, but then she came through just towards the end of that. And then she had a case that she wanted to talk about and discuss. And so, I listened and then we contributed to that in a similar way that we did to mine." (P9)

AC also experienced some disruptions to sessions that were classed as technical issues, involving hardware and aspects of the online system including:

- Internet connections (especially from laptops)

- Microphone and speaker volume

- Fluctuating sound quality

- Cueing to speak (and pressing a button to speak)

- Having only one person able to speak at a time

P2 gives an example of such frustrations:

"One was just around the volume. So, we had difficulties being able to hear people. And the second thing was that the more areas we had on, the more likely one would 'fall over' and that was really frustrating, particularly I think in some of the management meetings we had. We set aside time to deal with issues and then couldn't make connections." (P2)

These types of technical issues are described further in the discussion of reviewing systems use (below). Although there were some barriers to online peer consulting, progressing through a series of sessions allowed the structure and process of online peer consulting to be incrementally refined, as next discussed. 


\subsubsection{Reforming the online peer consulting sessions}

A key aspect of the Engaging stage is the process of ongoing reflection on peer consulting actions planned and taken by $\mathrm{AC}$, and the anticipated and experienced value of online peer consulting for AC. AC reviewed each session as it took place, and revised the structure and format of subsequent sessions as needed. Review typically occurred at the end of a session, variously described as the session reflection, debrief or review. Each review showed that AC gained value from the session(s) in question, gained insights into how the sessions could be reshaped to deliver additional value, and how the online system could better support future sessions. Key aspects of process and general session structure developments are described in this sub-section.

\section{Session preparation}

When considering the value derived from online peer consulting sessions, AC highlighted the importance of preparing for each online peer consulting session as they would for a face-to-face or phone consultation, finding that "we just need to go back to being prepared for our supervision as we would normally". (p4) Formalising session preparation and discussing rules or norms for the series of sessions (Akkerman et al., 2008) was therefore an important part of reforming the sessions. AC began by establishing the understanding that sessions would begin at a predetermined time, and unless urgent and/or critical work arose all were expected to be logged into the system, have any needed notes and resources and be ready to engage in peer consulting by the session start time.

Part of this preparation involved thinking through their client portfolio and current issues prior to a session, and being prepared to discuss a case with the group. This enabled them to gain additional value from a session by discussing cases that were going well, as opposed to only those with issues that needed attention, and gaining individual and collective insights from the peer consulting group. As the sessions progressed and individuals gained more experience with the format of the online peer 
consulting sessions and with Chatterbox, they were able to jump in and present a case for discussion when no one else had pressing issues:

"I think the idea of actually being more prepared for that supervision session. That one of us needs to say 'I'm going to present a client' on that day. Talk about the issues collectively and what's coming up for people. I mean, [P11] kind of jumped into the space and did that, while the rest of us were becoming more familiar with the technology, I guess.” (P4)

At times, members of AC let their colleagues know that they had a case issue to discuss prior to a session, either through a chance face-to-face conversation, a phone call or by emailing some brief case notes to others. After gaining experience of an online peer consulting session, $\mathrm{P} 2$ reported actively encouraging others to share case notes, or as much case detail as they could, before a session to see if this would lead to a richer discussion. This did occur in later sessions, as participants had reviewed similar cases that they had encountered and formed initial ideas and prepared some notes prior to the session.

\section{Session structure: The Hewson Triangle}

As discussed above, AC applied the Hewson Triangle (see Appendix E) to structure the case presentations and discussions within each session. This required some AC staff to undertake additional session preparation, to ensure they understood and could apply the Hewson framework and participate in the sessions. P9 emailed details of the framework to others prior to Session 1 and also presented and explained the framework and how it could be used within that session:

“...we weren't strictly following a Daphne Hewson type of model, I think, although certainly elements of that were present. And so I would have covered some counsellor aspects and gone back to looking at the three sides of that triangle and at those aspects. I must confess I didn't have the triangle in front of me and I didn't go around that systematically. But, I think as a matter of course certainly some of that model and observations was covered in the course of that." (P9) 
As AC gained experience using this framework to structure case discussions, they developed more ideas about how to apply it. Each presenter did not cover all 12 aspects of this framework, but selected the particular elements most relevant to their particular case or case issue and therefore most beneficial to discuss. This allowed sessions to remain flexible and for the case presenters to adapt the framework to their own style of presenting and learning.

AC made several observations as they gained more experience in using the Hewson Triangle. Firstly, they noted that having the framework itself displayed on the screen as a case presenter talked through various aspects of their case would enable others to 'see' the process as they moved through it, which they found to be beneficial. As a group, they found that the framework allowed them to cover the important aspects of a case while the presentations became more succinct and focused.

$\mathrm{AC}$ found that in a one hour session discussing any more than two cases meant that the level of detail and depth required was not reached, lessening the value of the discussion to the presenter and the group. If more than two participants had a case to discuss, priority was given to those who were most isolated within $\mathrm{AC}$, and an effort was made to discuss others if time permitted.

After the first two sessions were held, the benefit of sharing the resources and ideas on practice-related issues was reflected on and they found that "it was a really good way of connecting and so hence we've made it a regular fortnightly activity" (P2). As discussed within the Familiarising stage, AC had planned to move to regular monthly sessions, but as they experienced the value of regular peer consulting the plan was reformed and the sessions remained fortnightly. At this time they reformed the sessions in other ways, including establishing a more formal role of session coordinator, and including additional structure to the sessions through initiating an agenda and exploring ways to record session minutes. 


\section{Session facilitation}

Early on in the series of sessions, the importance of facilitating sessions was realised, and AC decided that "there'll be one person that is our coordinator, and they do the organising, and in a sense, calling the sessions" (P9). The introduction of this role falls within the description of coordinative activity (Akkerman et al., 2008), and is another step towards formalising the sessions. Although one participant initially adopted this coordinator role, others took on some supporting coordination tasks when necessary. Initially, this role was undefined and centred on "general communications", for example announcing the date and time of a session. As the sessions progressed, this role grew to cover activities before, during and after each session took place:

"My coordination role at the moment still tends to be
setting up conferences, conference times and
encouraging the counsellor to be able to share case
information prior to that if they are happy to get
themselves organised to be able do that." (P2)

The coordinator undertook several tasks prior to each session:

- Reminding participants that the Hewson Triangle was the organising framework for case presentations, and directing them to further information

- Checking, usually via email, that participants had the system running and were able to access the online conference room

- Assisting peers with any difficulties in setting up, accessing or using the system

Another key coordination activity was what AC termed "the call for cases to be considered" (P2). Here, the coordinator asked who had a case or specific case issue that they wished to consult on at the upcoming session. The call for cases assisted with session planning, including the timing. The coordinator then encouraged presenters to provide others with some case background prior to the session. When case information was shared in this way, the coordinator led by example, reviewing the material, gathering ideas and writing notes to share within the session. These tasks may also fall within the realm of a facilitation role, as facilitation of participation can be broadly defined to include tasks before and after a learning or communication event (Kimble \& Hildreth, 2005). In a more general sense, an 
important coordination role was encouraging members of $\mathrm{AC}$ to be involved in the sessions, by communicating the purpose of the sessions in an effort to "build some people's motivation to make a commitment to connecting up and participating." (P2)

As with many online facilitators of learning, the coordinator role at $\mathrm{AC}$ was also active within the sessions. After becoming more familiar with the systems functions, the coordinator used the text facility to post an agenda for session 3. This gave additional structure to the session, as all participants could see at a glance what was to be discussed. As the sessions progressed, this proved to be a valuable practice as on occasion, participants joined a session part way through and easily reviewed the session agenda with minimal interruption. In keeping with the flexible nature of AC, the agenda was sometimes modified to accommodate those with the most urgent needs. It fell to the session coordinator to facilitate the prioritisation of cases and issues, in consultation with those present.

Following a case description, the presenter usually facilitated the responses and discussion from the consulting group. The session coordinator was also prepared to facilitate discussions, and through the reforming activities was tasked with facilitating a round of responses from each participant to ensure that all perspectives on an issue were aired. Bernard et al. (2000) described this process as 'weaving', necessary for maximal participation:
"...the facilitator, that would be [P2], would ensure that every body responded once in the first round so that we didn't have some one responding and then having a pause, and then responding again before every one had a chance to say something." (P2)

\section{Additional session roles}

To extract additional benefit from online peer consulting, AC explored ways the key points of a session could be shared more widely, to also include those unable to attend a particular session. A popular option was to reshape sessions by introduction of the minutes secretary or session scribe role, to be initially undertaken by the session coordinator: 
"To be able to record the key ideas, or key suggestions or whatever. I think that would be really useful, that people do have access to some key learnings. It would be nice to have some reflections upon what was discussed and a record of those that people could go back to and use." (P2)

Although this role had potential to add additional benefit to AC, and was discussed at various times during the series of sessions, it was not actioned. Participants instead kept their own personal reflections and notes of the key points of each session.

\section{Widening the consulting group}

Complimenting the reforming of the structure of sessions, AC discussed how to include additional staff in future sessions, from two perspectives. Firstly, they noted that the number of participants in each session was increasing as existing staff came on board with the programme. The second perspective relates to the organisational change AC was undergoing, specifically expansion in the form of initiating new regional offices. New offices bring new staff into the AC team, and the session coordinator and other key participants realised the need to be able to easily include additional staff in the online peer consulting programme. Many studies found both positive and negative effects of including additional members in an online learning community, for example McDermott (2000) described the tension that emerged when newcomers to a community disrupted established patterns of interaction.

One key issue raised by AC was how many peers could be involved in a session and where participant limits lie:

"I'm interested just to test to see if there's any limits of numbers who might be involved. So it would be great at some stage for us to have the [Region 3], [Region 1] and [Region 4] office and [Region 2] all connected at once and see how it might work then." (P2)

Bernard (2000) also addressed this issue, and summarised collaborative online learning literature that found "problems related to the distribution of information and resources in a context where group members communicate via synchronous 
conferencing are increasingly amplified and complicated as the group size increases" (p. 267). Key concerns centred on what AC could accomplish within a one hour session if the number of participants was greater than five. Factors considered include:

- How many cases could be presented within a one-hour session

- Complexities of prioritising cases with a greater number of participants

- Impacts of queuing for a long time before offering a comment or response

- Whether the overall effectiveness and value of a peer consulting session may diminish with greater numbers

The session coordinator and other key participants discussed the option of conducting regional sessions, including one or two isolated peers from other regions. Although this situation did occur several times, it was not explicitly planned. Expanding sessions from a three/four to five participant group did not have any negative effects on sessions: "we thought the larger things got the less functional it would be. And I've not found that at all." (P1)

The strength of the relationships between individuals and the wider AC group also emerged as an issue impacting on session success/value. Communicating online and relying on mostly non-verbal cues to relay meaning can be less problematic when relationships are strong, and parties to the communication have some face-to-face shared history built up over time (Akkerman et al., 2008):

\footnotetext{
"Because I have a face-to-face history with the other staff, I feel like I can read them fairly well when I am talking to them. Whereas it might be different with a new staff member who I didn't know." (P1)
}

However, some staff did have pre-existing relationships with those staff members new to AC as P1 stated "we do have a history with each other at different levels." Although participants reported that these relationships, both socially and professionally, were often not yet as strong as those between existing AC members. Therefore, when planning to include additional staff in the sessions AC was aware that they needed to rely on the non-verbal and limited verbal cues present, and to be conscientious about checking the meaning of any comments that they were unsure of. 
$\mathrm{AC}$ also felt that the overall process of a session may not unfold as seamlessly as usual, with conversations being more "staccato" at first, and they were prepared to slow the sessions down until new members were comfortable with the session format. Despite potential difficulties in involving additional staff in the sessions, whether existing or new staff, it was generally agreed that they would bring a new and interesting perspective to peer consulting, and widen the scope of advice available within AC. As P1 noted, "the more comfortable we were with each other the more effective it was and I think the more we understood." Therefore, as part of reforming the sessions participants became aware that although additional structure was needed, the online peer consulting programme needed to remain flexible in order to adapt to new membership.

Through reviewing the sessions within the consulting group, and later reflecting on their experiences of online peer consulting AC articulated how online peer consulting compliments other forms of professional development that they engage in:

"I see a real difference between this, what I'd classify as casework supervision and what I do with my faceto-face supervisor. In that, a lot more of what I talk about with him is the interface between what I am doing, and who I am. Whereas, I find that the conversations that we have in supervision are a lot more focussed on my relationship with the clients." (P1)

P1 clearly shows an understanding of the nature of online peer consulting, the case focus and how the sessions add to rather than replace other professional development activities. Overall, reforming the series of sessions and adding additional structure led AC to view online peer consulting as falling within their formal professional development processes, as opposed to informal practice related discussions:

"As yet we don't have a formal supervision contract here, so [online peer consulting] is not as formal as my other supervision relationships. But, I think it is tending towards being a formal supervision or peer consultation relationship, just by the nature of its regularity and by the nature, and sort of the structure that will go around it eventually." (P9) 
Several factors contributed to this:

- The focussed nature of the sessions (as described within the performing activity above)

- The evolving structure of sessions

- Inclusion of the session coordinator role

- Scheduling sessions at regular (fortnightly) intervals

As this discussion has shown, the series of sessions was reformed in a variety of ways so as to deliver the most value to members of AC. As this reforming took place, $\mathrm{AC}$ also reflected on their use of the supporting system and other information and communication technologies as next discussed.

\subsubsection{Reviewing systems use}

Completing the Engaging stage is the ongoing reflection on and review of the use of Chatterbox and other ICTs to support online peer consulting. As AC reviewed and reformed the structure of the sessions they also reviewed their use of the supporting system, identifying the issues and behaviours that supported and inhibited the peer consulting sessions.

The start up phase of $\mathrm{AC}$ allowed more flexibility in the process and overall structure of the online peer consulting programme, but the lack of a uniform and reliable technical infrastructure hindered programme progress at times:

"We have been having teething problems with our IT infrastructure that may have placed barriers in the way also.” (P9)

As previously described, AC is made up of regional offices, with two, three or four staff within each office and one region that has one staff member. When participating in online peer consulting, those staff with co-located colleagues chose to share one computer, and pass the microphone among them. Generally, this arrangement worked well although there were a few unanticipated issues that emerged. For example, in Region 2 responsibility for setting up the system, logging into the online 
conference room and testing the connection was adopted by P3. Others in Region 2 raised concerns about who would take this initiative if $\mathrm{P} 3$ were not able to attend a session, as they did not feel that they knew the process well enough.

Two of the more isolated staff experienced some initial difficulties when joining a session with co-located peers. Early on in the sessions, when an isolated person was speaking, their (co-located) colleagues in another region would comment on what was being said as they are listening. Therefore, the isolated participant missed out on some of the discussion:

"I think that just to begin with it was the feeling of not exclusion, but just... sort of a question about, 'have I missed some thing here?' because people would come back in with a slightly different tone. And that just made me wonder having raised that question, had I missed part of the discussion or comments that I wasn't party to." (P2)

Another isolated participant relayed a similar experience:

\begin{abstract}
"What I noticed is that I was on my own in this office, and at any one time there were three of them there. What would happen some times is they were using a desktop microphone and because the clarity was quite poor, there would be people in the background asking questions or responding to something, and I wouldn't be able to hear them. And so, that was quite difficult at times because I would miss chunks of the conversations and the interactions and so therefore I would find it difficult to cue in. Things like, I didn't know if the question that I wanted to ask, or the thing that I was wondering about had already been raised or asked.” (P9)
\end{abstract}

P9 describes a situation where one participant is speaking, and other co-located colleagues are also contributing, but are not close enough to the microphone for others to be able to hear them clearly. Such situations illustrate the key difficulties that arose with co-located peers sharing a computer and microphone when consulting with one or more isolated peers. 
Reviewing use of the system within the consulting group and through individual reflections led to a better understanding of such situations from both the perspective of the co-located and isolated participant, and as a group AC became more conscious about using the microphone for all communications during a session, no matter how insignificant an idea seemed at the time. Developing norms of communication in this manner is another activity that aligns with the description of coordinative activity given by Akkerman et al. (2008). This resulted in all parties to a consultation being privy to the same information, and moving through the process together:

"They became more conscious about making sure that they used the microphone to share any of their asides or, you know comments... I did appreciate their efforts that they did work to have their finger on the button whenever they wanted to talk." (P2)

Some members of AC noted that it would be less problematic if there were one participant per computer, and although future plans for this were discussed it did not eventuate during the period of this research. Situations such as this are learning steps and part of the process of developing the online peer consulting programme. CecezKecmanovic (2002) found that as organisational members increasingly use a technology, they are able to express their needs of the technology more clearly. This may stimulate further improvements and changes to the particular technology, or changes to organisational processes to ensure that the technology becomes an integral part of peoples work processes.

Congruent with the findings of Cecez-Kecmanovic (2002) AC experienced a period of adjusting to the communications format of the online system. Many AC staff had no prior experience of using online conference facilities and therefore took varying amounts of time to adjust to the online format, while others did not feel comfortable consulting online at the conclusion of the series of sessions. The disruption to the flow of their communications in the online environment was highlighted. Several factors led to such disruption, including the physical absence of peers, and the way that the supporting system structured their interactions. For example, having to push a button before they could speak was frustrating at first, and some people forgot to do this and needed reminding. In addition, having only one person speaking at a time, 
while others queued for their turn took some adjusting to. Some participants with colocated colleagues found adjusting to this easier, as the physical presence of others helped to mitigate issues. Generally, once AC became more at ease with the communications format they gained value from the sessions:

"As the person presenting the case I found the process very useful, i.e. in terms of consulting re: my work, I found it at least as useful as a face to face consultation. Particularly once I had grown used to the Chatterbox format; not having the dialogue feel of natural speech took 5 minutes or so to adjust to." (P9)

Although P9 took a minimal five minutes to adjust to the online communications format, others still struggled with it in sessions 5 and 6 . A related aspect of the communications format that $\mathrm{AC}$ experienced difficulties with at first was the absence of non-verbal or visual cues, such as facial expressions, body language and movements. The nature of AC's work was seen as a contributing factor here, P11 noting "...part of it is not being able to see all the non-verbal cues. I mean, we are counsellors so all the body language and all of those things are a natural part of our communication processes. So [online communication] is a new process for us." (P11).

Participants described that they couldn't 'see' how important or urgent an issue was to a colleague, and noticed the absence of cues from people's eyes and general bearing or body language. Usually, they would read the 'unsaid' signs and pause in their discussion to let others respond. Also, they could not 'see' whether a peer was understanding or processing the intended meaning of what was said, and found it difficult in the online environment to know when to clarify an issue or pause to receive questions or responses from the consulting group. Dennis \& Valacich (1999) frame this as symbol variety, the ways in which information can be communicated via Chatterbox. A lack of non-verbal and some verbal cues in this online environment resulted in diminished social presence, and the need for AC to alter their communication practices. On reflection, participants viewed it as part of their professionalism to ensure that their meaning had been received accurately by others, and that they had in turn correctly understood the meaning of comments given to them. Similar to the findings of Bernard (2000), members of AC noted early on in 
the series of sessions that the absence of non-verbal cues gave rise to the importance of more active and focused listening:

"You're not able to pick that up if the person is at the end of another computer miles away... So, a whole lot of the intuitive non-verbal stuff you're not able to pick up on as accurately. But at the same time the discipline around listening carefully becomes more important." (P4)

"And also, as sessions went on, the 'strangeness' of not having visual cues, I sort of got used to that as well. One more thing I found is that I did tend to do a lot more focusing, you know actually on what people were saying, because there weren't all those other cues available to distract me." (P2)

Participants therefore relied on voice-based cues a lot more in the online environment, highlighting the need to listen intently during a session. However, when reviewing their system use participants noted that relying on voice cues was often problematic, due to variations in sound quality brought about either by speaker and microphone settings or quality or the quality of the online connection between regions. They experienced sporadic difficulties in hearing others, or in others hearing them, but referred to this as more of an annoyance than an issue that severely impeded a session. At times, repeating comments or questions due to poor sound quality slowed a discussion, but participants were able to persevere with this and complete the session. Many participants described adjusting speaker and microphone volumes before or during a session as one exercise in getting used to the online format. $\mathrm{AC}$ was able to more easily cope with the technology-related challenges faced at the Engaging stage due to the strength of their existing relationships and the shared history they had accumulated (Riverin \& Stacey, 2008).

Despite needing to adjust to the communications format and gain experiences with the supporting system, most participants found that the overall process of online peer consulting was not so different from face-to-face consulting. They noted that the case-based format was similar, if not exactly the same, P4 finding that "the process was similar to the way we would discuss it... we are using Chatterbox, but it didn't 
change the way we kind of looked at things, discussed things." (P4). One main difference consistently felt by participants was the increased formality of the sessions (as previously described). This was brought about, in part "because we're on the computer and there's microphones and things in front of you, you feel quite business like so you rip into it, we ripped into it quite quickly." (P1)

As participants reviewed and reformed the series of sessions, they also reviewed and extended their use of the supporting system. For example, when participants added additional structure to sessions by including a session agenda, they also explored ways that the functions of the system could best support this. One participant describes how the coordinator used another function of the system to do this:

"[P2] took an agenda and I didn't realise that he actually typed it up and it popped into the corner of the computer screen, so that was really cool to realise that that was possible." (P1)

Seeing the coordinator using the text function of the system sparked the curiosity of others, and they then tinkered with this during the session, the more experienced participants assisting those less familiar with this function. Wenger, McDermott and Snyder (2002) support this approach to developing technical skills through participating in online collaboration, and include it as an effective way to become familiar with community tools.

Another example of participants extending their use of the system congruent with changes to the structure of the sessions was when participant P9 explained the Hewson Triangle to others during a session. After deciding that it would be useful to have the figure up on the screen as they talked through the framework, P9 and P2 explored how the system could support this. They successfully displayed the figure for others to view, and reviewed their use of this function and discussed other possible uses. 
Participants also discussed using the text function to support the minutes secretary role (as described above) and keep a record of each session. Although the secretary role was not formally established and thus the text function not used for this purpose, by participants reviewing their systems use and discussing potential future uses they improved their understanding of the system and how it could best support online peer consulting at AC.

It is important to note that participants not only reviewed their use of the supporting system, but also reflected on their use of other communications channels and media before, during and after each session. Prior to a session participants sometimes let others know that they wanted to present a case or discuss a particular issue. This was usually via an email message, or a face-to-face or phone conversation. At times, participants were unsure who was able to attend a session and a series of phone calls would be held to confirm participant availability. If participants experienced difficulties such as accessing the system or poor sound quality they would revert to a different channel, such as the telephone, cellular phone or email. This media switching increased as the importance of gaining timely advice increased.

Reviewing their use of ICTs was an important activity that existed alongside reforming the series of sessions. Through analysing their use of the various functions of the system, participants were able to better understand their experiences of online peer consulting, as well as explore ways to use the system to better support the changing nature of the sessions.

\subsubsection{Engaging stage summary}

Within the Engaging stage, participants put into practice the online peer consulting plan developed through previous stages. As they progressed through a series of six sessions, participants reviewed the overall process and structure of sessions and their use of the supporting system and other ICTs. Subsequently, participants 
incrementally revised the structure of the sessions and extended their use of the supporting system.

As participants experienced online peer consulting they realised the benefits of gaining varied perspectives on their practice and gained an understanding of how this online professional development activity could compliment other, more traditional forms of professional development they engage in. By accepting online peer consulting in this way, participants moved towards accepting the sessions as an organisational activity, named the Crystallising Point. This is the first step in establishing online peer consulting as a long-term organisational practice that is part of day-to-day work. The Crystallising Point completes the Engaging stage, and propels AC to the final developmental stage of Embedding.

\subsection{Embedding at AC}

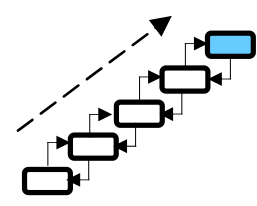

At the Embedding stage, AC began to integrate their online peer consulting programme with existing professional development practices. They undertook several tasks in order to re-evaluate the online peer consulting concept developed and refined through earlier stages, as well as realign professional development practices to encompass online peer consulting. Once this process was underway, $\mathrm{AC}$ also turned their attention to upgrading technical infrastructure to accommodate additional online tools needed to support long term online peer consulting at an organisational level.

For clarity of description, I have organised the observed actions of AC at the Embedding stage within the activities of Cementing the online peer consulting concept and Advancing technical infrastructure, and each is elaborated on within this section. 


\subsubsection{Cementing the online peer consulting concept}

"I think [distance] is one of the largest threats that we actually face. One of the major things is support for staff and for staff to actually be able to deal with professional issues as they arise. But the other bit is for the staff to maintain relationships." (P4)

As AC developed as an organisation, they became acutely aware of the challenges of operating across distance, as a "small but widely spread" organisation (P9). As part of the Embedding stage discussions, AC identified professional support and relationships as central to fostering the community atmosphere to which they aspired, and online peer consulting as one way to achieve this.

At the early stage of integrating online peer consulting with existing professional development practices, AC reflected on their initial and evolving conceptualisation of online peer consulting and professional development in general. Specifically, they reviewed outcomes of previous stages (Scoping, Shaping, Familiarising and Engaging) alongside other organisational professional development practices and began to develop a vision for long-term organisation wide online peer consulting.

As their concept came together, $\mathrm{AC}$ discussed ideas for how and where online peer consulting aligned, or potentially aligned, with other professional development activities. The key aspects of these discussions are elaborated on through this subsection.

\section{Levels of professional development}

$\mathrm{AC}$ views professional development in terms of three distinct yet interconnected levels:

- Individual: "specific training issues" (P9), the particular areas of knowledge, skill and/or interest an individual needs/wants to develop.

- Regional: "targeted training" (P9) for all members of AC in a particular region, encompassing courses, conferences, readings and group consulting. 
- Organisational: any opportunities for the whole organisation to upskill in an area, for example a new/ modified practice, policy, law.

Viewing professional development at $\mathrm{AC}$ as falling within these three levels is part of AC "making that shift from thinking just regionally, to thinking in terms of the wider organisation" (P9). When discussing organisation-wide professional development, P9 took a broad view, stating that "supervision has to be a whole lot of things. And there is a continuum from very informal to kind of formal" among which different peer consulting, peer supervision and professional development activities were placed. At the informal end were processes through which AC gained insight into certain areas of their practice, and at the formal end of this continuum was the supervision contract and sessions they participated in. Safe practice at AC was supported by taking a "sampling" of different forms of professional development within this continuum.

\section{Vision for online peer consulting}

All stages of the development of their online peer consulting programme involved the creation, review and redevelopment of AC's online peer consulting concept. In order to Cement the concept into the organisation's culture and practices, AC elaborated on particular aspects that are described here.

In accordance with their views of formal and informal professional development, online peer consulting was placed alongside formal supervision:

"One thing that we haven't discussed, and we probably will, is whether there is a more formal supervision contract, maybe contract is not quite the right word, supervision agreement or even policy, around the sessions. So that, maybe it brings these sessions even more into line with some of the more formal supervision relationships." (P9)

Reinforcing the view of online peer consulting as formal professional development, the role of Training Coordinator adopted by P2 "indicated that Chatterbox sessions will become more central to the way we fulfil our casework supervision 
requirements." (P2) Enhancing professional development in this way matched with the needs of members of AC, specifically to "widen the pool" (P3) of those available to consult and "open up" (P1) professional development across the whole organisation.

Other elements of the vision for online peer consulting at $\mathrm{AC}$ highlighted at the Embedding stage were:

- Providing the opportunity to consult on all types of AC's work, and nonurgent issues

- Timetabling online peer consulting, and ensuring that it is scheduled regularly, as part of routine

- Creating the space for online peer consulting, including the time and (virtual) place

- Accepting, encouraging and supporting different levels of participation, from listeners to presenters and coordinators

- Introducing shared and revolving roles, such as regional session coordinators

Along with full support from AC management team, these elements comprise the final aspects of AC's continued, organisation-wide online peer consulting concept.

\section{Integrating organisational professional development processes}

For online peer consulting at AC to be sustainable long-term it must be part of the organisational professional development programme. Although AC was still developing their overall approach to professional development at the time of this study, they described online peer consulting as fitting into all of the three professional development levels (individual, regional and organisational), whether an individual consults with a colleague who has expertise in a particular area, or resources are made available for the professional development activities of all staff.

The limited opportunities for AC staff to meet face-to-face was also highlighted, and online methods of professional development identified as a way to "provide a bridge" (P1) between physical meetings and maintain the momentum of professional development initiatives. This blended approach to professional development involves 
the integration of the use of technology with more traditional communication mediums, including face-to-face, phone, email, online and other digital media (Fink \& Disterer, 2006; Green \& Cifuentes, 2008)

One such situation at AC reported by P9 was a conference that three staff had attended at which they learned of a new professional framework that they considered introducing to AC. They formed a project group, and as they were separated geographically they planned to meet online fortnightly to discuss and/ or plan actions and progress. Although these members of AC could still have worked together on this project without online support, they realised that "if we're relying on face-toface meetings, it might not happen so easily" (P9), and is likely to have taken much longer and used additional resources.

Researchers report that when introducing a new technology, it is important to assess the effect of that technology on existing work processes and practices (Munkvold, 2002; Al-Qirim, 2007). In addition to face-to-face professional development activities, AC also recognised the potential of online peer consulting to enhance other organisational processes. Reflecting on earlier discussions of ways to accommodate new staff in online peer consulting sessions as the organisation expanded, P4 questioned whether online peer consulting could become part of the induction process for new staff. Specifically, sessions could be used for:

- Assessing the professional development needs of new staff

- Providing professional support to new staff

- Establishing relationships with those staff they had not met face-to-face

- Delivering interim professional development before scheduled face-to-face events (such as conferences, organisation-wide meetings, and external supervision)

Successful implementation of online peer consulting technology at AC would be evident when new staff are introduced and trained to use it as a matter of course (Ramsey \& McCole, 2005), and integrating the induction process with the online peer consulting process would support this. 
At the Embedding stage, AC did not generate a definitive picture of how online peer consulting compliments and aligns with other professional development processes. However, the ideas generated through these discussions helped to understand aspects of how new and existing practices could come together to fulfil some of the key needs of staff. There were still many unknown elements to be addressed before AC could fully cement their online peer consulting concept. P9 described the issue of how to make resources (including books, journals and e-journals) available to all staff, and P4 questioned how to accommodate the different learning styles of AC staff, as some preferred a highly structured programme and/ or environment and others learned best in unstructured, fluid and flexible situations.

Another key set of questions raised centred on the ICT investment required for continued online peer consulting, as next outlined.

\subsubsection{Advancing technical infrastructure}

"We employ people who are passionate about what they do, but very few of them are passionate about technology." (P9)

AC is passionate about their work and developing their practice. As ICT (specifically Chatterbox and Blackboard) enabled them to do this more often, within a larger group of peers, they accepted these online ICTs and engaged in online peer consulting sessions. Through their experiences at the Familiarising and Engaging stages $\mathrm{AC}$ were aware that for online peer consulting to become a sustained, longterm professional development activity appropriate ICT support was essential.

$\mathrm{AC}$ was in the start-up phase and still expanding, therefore they were working through additional issues such as keeping their technology infrastructure developing at the same rate as their staffing, regional offices and processes. In NZ, the Government recognises the difficulties facing SMEs when it comes to technology investment, and also reports that ICT is a vital component of these organisations, 
especially those that are geographically distributed (MED, 2007; Massey et al., 2004). When assessing the kinds of technology they could invest in, AC took into consideration staffs' attitudes to and comfort levels with technology, and the challenges faced at previous developmental stages (see section 5.4.3, above). Generally, ease of use and accessibility was a priority:

"We need to make sure that whatever piece of technology we're using or piece of software or whatever, that it's easy. And they'll use it only if it's easy." (P9)

One integrated system, or set of systems with centralised access was discussed as one way to simplify to technological aspects of online peer consulting. Lack of access coupled with technical difficulties is a commonly reported barrier to participation to online communities (Riverin \& Stacey, 2008). P2 provides a typical comment, stating "I'd like to have a way of being able to do Chatterbox and email and Blackboard all in the one facility." Ideally, this could encompass:

- Chatterbox and Blackboard (or similar online conferencing/learning management systems)

- Document/ file repository

- Email, contacts and calendar

- AC's website (or future intranet)

- Links through to e-journals and other external resources

AC did not have the capacity to provide such functionality with existing infrastructure, and therefore could not engage in reconfiguring systems as described in Chapter 4. Instead, AC planned to work with their IT partners to introduce additional technical infrastructure to provide this kind of access to systems/technology at an appropriate time in the future.

Advancing technical infrastructure is deemed complete when an organisation has a technical infrastructure that has been reconfigured (or extended) and is capable of supporting long-term online peer consulting. Through discussing the ways in which AC could advance their technical infrastructure to support continued online peer consulting, they determined what to prioritise, improved their understanding of the 
technical requirements and possibilities and were more prepared to work with their IT partners to make the necessary changes. This is a significant point in the Embedding stage, as a barrier to understanding can exist, where there is a misunderstanding of the consequences or potential benefits of proposed new technology (Ariss et al., 2000). At this point they made a firm commitment to continue with some form of online processional development, and understood that they would need to introduce additional technology and reconfigure existing technology in order to do this.

\subsubsection{Embedding stage summary}

AC progressed as far towards Embedding online peer consulting as their organisational situation allowed. By beginning the activities of Cementing the online peer consulting concept and Advancing technical infrastructure AC synthesised the experiences and learnings gained during all stages of peer consulting transition into an effective organisational practice of online peer consulting. An output of this was a refined vision for regularly bringing staff together 'virtually' for practice related peer consulting.

Taking this vision and discussing the long term infrastructural requirements for supporting continued peer consulting enabled $\mathrm{AC}$ to gain further insight into the needs of their staff and identify technical options for fulfilling such needs and supporting increased organisation-wide engagement. Once AC reaches the point in their organisational development at which they are ready to invest further in technical infrastructure, it is likely that the knowledge gained at the Embedding stage will be applied, and online peer consulting can then be firmly established as a professional development activity at AC.

Although AC did not fully complete all activities of the Engaging stage, they made a firm commitment to continue with online peer consulting, the programme is, to an extent, embedded within the organisation, and they are prepared to advance 
infrastructure in the future. Thus, AC passed through the Concretising Point. This signals the conclusion of the Embedding process, and ends peer consulting transition at $\mathrm{AC}$.

\subsection{Summary of the Story of AC}

As an organisation that values openness, inclusivity, high professional service standards and fostering a sense of community among geographically distributed staff, $\mathrm{AC}$ undertook to explore alternative ways to enable staff professional development. A key factor driving this exploration was a desire to reduce both professional and social isolation among staff, through the provision of an environment within which regular and ongoing group peer consulting could take place. Specifically, this chapter has given a detailed analysis of their journey through the stages of the Model of Peer

Consulting Transition. As this journey was unique to AC, the particular activities described in this chapter vary somewhat from the generalised model of Chapter 4 , as depicted in Table 11. 


\section{Model of Peer Consulting Transition}

Stage Name:

Defining Stage

- Mapping current peer consulting activities

- Documenting current ICT support

Stage Activities:

- Developing the online peer consulting concept

\begin{tabular}{|l|l|l|}
\hline Stage Name: & Structuring Stage \\
\hline Stage Activities: & - & $\begin{array}{l}\text { Planning for a series of sessions } \\
\text { - }\end{array}$ \\
\hline Training on systems use
\end{tabular}

Table 11: Components of AC's Peer Consulting Transition

\section{AC's Peer Consulting Transition}

Scoping Stage

- Determining current peer consulting activities

- Discovering ICT support

- Developing the online peer consulting concept

Shaping Stage

- Honing the online peer consulting concepts

- Selecting and setting up systems

Familiarizing Stage

- Training-in-action

- Planning online peer consulting sessions

Engaging stage

- $\quad$ Performing formal online peer consulting

- Reforming the online peer consulting sessions

- Reviewing systems use
Summary of Differences

At the first transition stage AC began preliminary Scoping of staff peer consulting and use of technologies. AC unearthed various aspects of their developing professional development and ICT use practices, but not to the degree that they were formally defined and documented.

Shaping stage activities built on the outcomes previously achieved. As knowledge of current practices and the potential value of new

(online) practices was gained AC honed their online peer consulting concept, and took action to Shape elements of their online peer consulting programme.

AC began a period of Familiarising staff with the purpose and process of online peer consulting through training-in-action. This resulted in heightened understandings and a collective experience on which to base initial planning activities.

Engaging in regular online peer consulting sessions resulted in AC iteratively reviewing the structure of the series of sessions and the nature of ICT support, and reforming the plan they worked to. 
Figure 8 below presents the stages, activities and trigger points that emerged as most prominent in AC's pathway to Transitioning Peer Consulting.

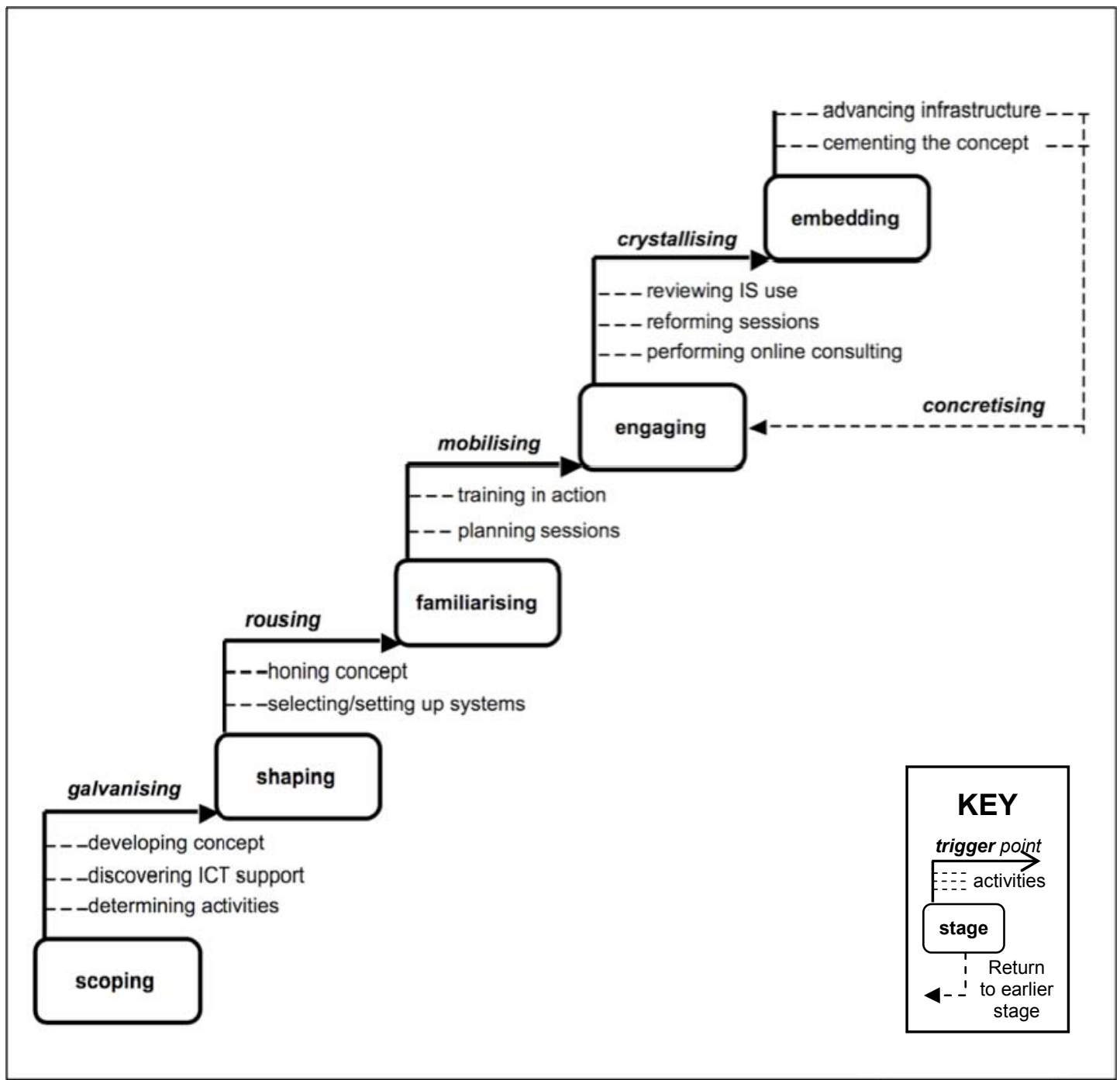

Figure 8: Peer Consutling Transition at AC

As a newly formed organisation that is still refining many operational processes and practices and developing their organisational infrastructure it was appropriate for AC to undertake some activities in a different order and/or to a greater or lesser extent than prescribed by the generalised model. For example, at the Familiarising stage AC chose to first experiment with online ICTs before outlining a plan for a series of online peer consulting sessions, and before they had a well defined vision for online peer consulting. Outcomes of peer consulting transition reported in this chapter 
suggest that MST was a useful lens for assessing the nature of online ICT resources that may best support AC's peer consulting activities. ${ }^{9}$

Chapter 6 provides additional discussion on the similarities and differences between elements of AC and CI's transitions, and the generalised model of Chapter 4. Many of AC's actions and decisions throughout peer consulting transition aligned with their flexible and open approach to new initiatives, and also reflected their early stage of general organisational development. Such elements of organisational structure and culture and extent to which they impacted peer consulting transition are analysed further in Chapter 7.

${ }^{9}$ See Chapter 8 for further discussion on the application of MST in this study 


\section{Peer Consulting Transition: The Story of Counselling Incorporated}

\subsection{Chapter Overview}

Counselling Incorporated (CI) is a N4P organisation distributed across NZ that provides a range of relationship and workplace counselling and education services in communities nationwide. Established over 60 years ago, CI has relatively stable and well defined organisational processes, policies and service offerings. Support staff administer the clinical and operational activities of approximately 170 counsellors (many of whom work part-time) from CI's centrally located Head Office. Their history of quality service provision distinguishes $\mathrm{CI}$ as an industry leader.

CI moved through many activities in their journey to transition elements of their peer consulting practice to the online environment. The Model of Peer Consulting Transition (as introduced in Chapter 4) provides a frame within which I have placed the actions of CI, each stage of development being distinguished by a number of key activities and a trigger point that combined to propel CI through the transition process to the Refining stage. As some activities span across stages, or are repeated at various stages, they are presented here where I observed them to be most dominant.

As previously described, I developed the Model of Peer Consulting Transition by generalising from the unique experiences of the participant organisations, $\mathrm{AC}$ and $\mathrm{CI}$. Therefore, CI's story of peer consulting transition, as presented in this chapter has some important differences from the model described in Chapter 4. Specifically, differences occur in:

- Titles of some stages

- Titles of some activities

- Order of some activities within stages

- Titles of some trigger points 
These are highlighted throughout the chapter and discussed in more detail as part of the chapter summary. For ease of comparison I have organised Chapter 6 in a similar way to Chapters 4 and 5, proceeding through four stages of the Model of Peer Consulting Transition, before finally drawing several conclusions and diagrammatically presenting their overall journey (in Figure 14).

\subsection{The Defining Stage}

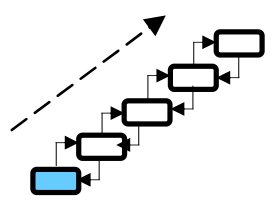

CI began their journey towards online peer consulting by assessing the current state of professional development processes, practices and capabilities. Key members of $\mathrm{CI}$ and I collaborated to define why an online peer consulting programme was needed, and developed an initial conceptualisation of what such a programme could look like. The main focus of this stage was mapping the peer consulting activities undertaken within $\mathrm{CI}$, as discussed in sub-section 6.1.2.

I have grouped the actions of CI at the Defining stage into the categories of:

- Developing the online peer consulting concept

- Mapping current peer consulting activities

- Documenting current ICT support

Each is described in turn within this section.

\subsubsection{Developing the online peer consulting concept}

In order to form an initial vision of what online peer consulting could mean for the target participant group, CI firstly discussed the potential of online peer consulting to promote greater connectedness among group members. Many participants experienced a lower level of connection and interaction with their peers than during previous years: "it feels like we haven't been as connected" (P16). Some stated it 
was irregular for one Manager ${ }^{10}$ to contact another directly, and examples of when they do interact for practice related issues were given as:

- The presenting of an issue centred on a 2-location, Family Court case

- The exchange of paperwork around a particular process

- Issues arising on the introduction of a new process/practice

- The presenting of an issue centred on a particular practice area (see Appendix F for additional detail)

Although CI experienced a diminished level of peer consulting, the participant group showed awareness of both the need and the value of discussing issues among their professional peer group:

"Well, I think it's really useful for all of us to be regularly communicating about all kinds of issues and for us to have a forum to be able to do that. I completely support that. I think that there's probably about a million different issues that we all have that we could support each other with, or discuss with each other, or maybe extend our practice." (P2)

As Lock (2006) described, this becomes even more essential when professional practitioners such as CI Managers are separated by distance.

Through a series of meetings two CI senior managers other Wellington-based participants and I explored the peer consulting situation within CI and outlined the need for introducing online peer consulting, key points of which included:

- Managers are geographically isolated from peers

- CI provided few opportunities for Managers to meet face-to-face

- Limitations (particularly time and finance) had prevented them gaining the benefits of consulting with peers

- A higher level of interaction (to discuss practice-related issues) was desired among the group

These and additional findings were compiled into a scoping document (see Appendix $\mathrm{G}$ for detail) that was distributed among the participant group for review. CI next

\footnotetext{
${ }^{10}$ Regional Practice Managers are CI's clinical leaders of practice throughout New Zealand, referred to as Managers in this chapter.
} 
moved to mapping current activities, and further refined their online peer consulting concept.

\subsubsection{Mapping current peer consulting activities}

Following the development of a working vision for online peer consulting, we collaborated to map the peer consulting practices undertaken by members of the Manager group. Firstly, the nature of peer consulting from both the consultee perspective of seeking advice from a peer and then from the consultor perspective of giving advice to a peer was explored ${ }^{11}$. Participants recounted recent peer consulting episodes where they had acted as both a consultee and consultor to one or many of their peers.

Exploring current peer consulting practices from the consultee and consultor perspectives showed:

- Consultation took place in an ad hoc, informal way between Managers and staff (or staff and peers) within the same office

- There was little consultation taking place between Managers/staff from different regions

- Managers met at twice yearly national face-to-face meetings

- Localised knowledge existed at both an operational and managerial level

- The frequency and duration of a consultation varied greatly depending on a number of factors:

- availability of consultee and consultor

$\circ$ urgency of an issue

o other activity going on within $\mathrm{CI}$ at the time of the consultation

Appendix $\mathrm{H}$ provides additional detail of the nature of the peer consulting process at CI.

Gaining an overview of the nature of peer consulting at CI provided an important foundation from which the second element of this activity was undertaken - the

${ }^{11}$ The terms 'consultee' and 'consultor' were coined by D. Keates (2003). 
mapping of the actual steps of the peer consulting process from the two complimentary perspectives. Generalising from the interview testimonies of participants, I grouped their comments into distinct but interrelated steps of the consultee and consultor perspectives of the peer consulting process, from which I generated a set of process maps, presented in Figures 9 and 10 and supported by Tables 12 and 13. 


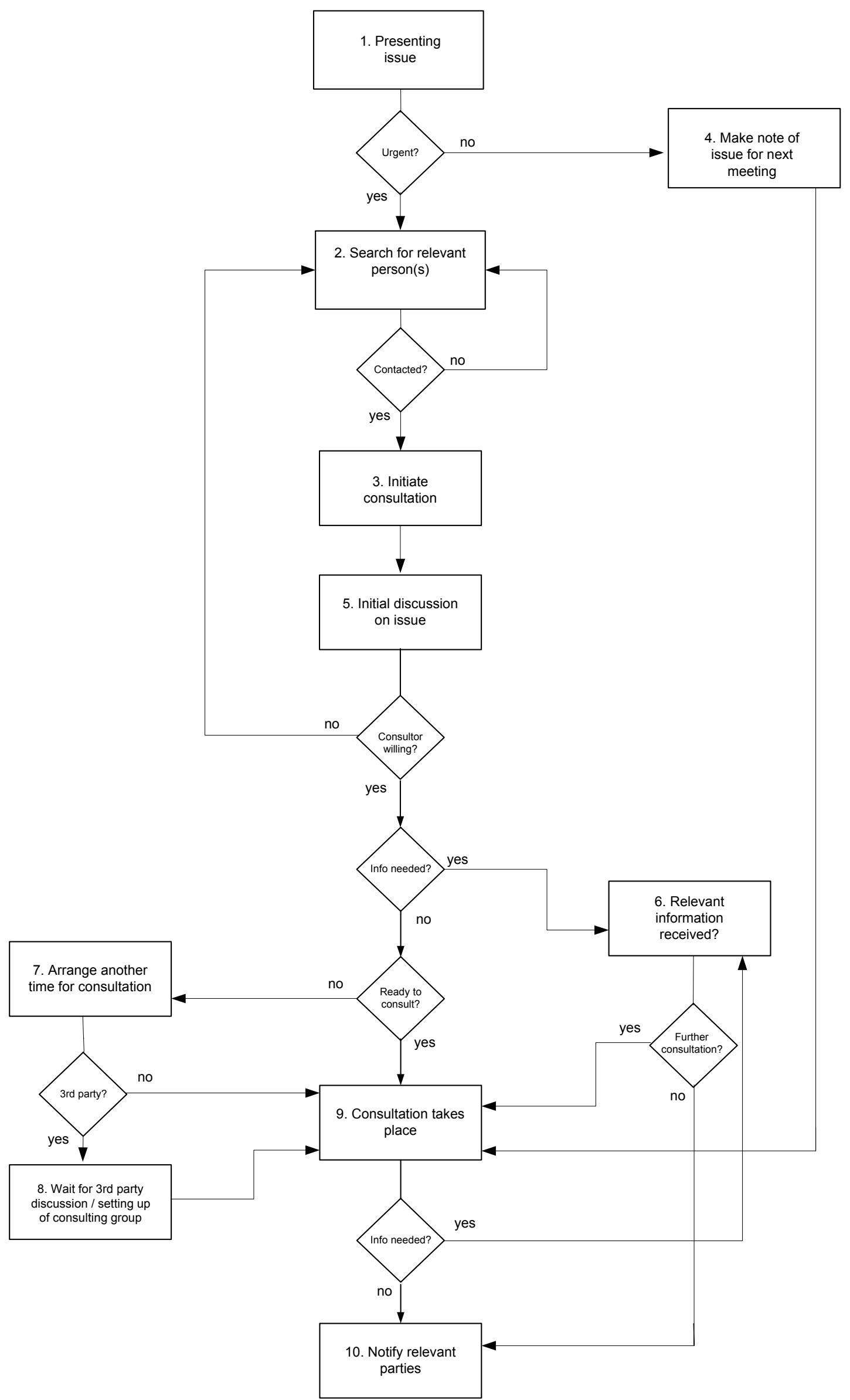

Figure 9: Integrated consultee process diagram 


\section{1: Presenting issue}

\section{2: Search for relevant person(s)}

\section{3: Initiate consultation}

\section{Make note of the issue for the next meeting}

\section{5: Initial discussion on issue}

6: Relevant information received

\section{7: Arrange another} time for consultation

\section{8: Wait for $3^{\text {rd }}$ party discussion / setting up of consultation group}

\section{9: Consultation takes place}

\section{0: Notify relevant parties}

The consultee is presented with an issue that they need advice on. After considering the issue, they identify the need for consultation with peers

If consultee immediately knows whom to contact, this step may involve searching for their correct contact details, work hours/days etc. If they do not know who they need to contact, this step involves asking around their office, ringing around peers at other offices, or sending a group/individual email.

When the relevant person(s) are identified the consultee attempts to make contact with the relevant consultor, and the consultation is initiated.

The consultee makes note of a non-urgent issue, to then be raised at the next relevant scheduled meeting.

An initial discussion takes place to establish the nature of the issue, whether the consultor is the best person to deal with the issue, and whether the issue is worthy of further consultation.

When a consultation requires the consultee to review additional information, it is received here.

If the consultation cannot take place immediately, another time is scheduled.

The consultee will wait while the consultor has a discussion with a $3^{\text {rd }}$ party to the consultation, or organises a consulting group (these events are described in the consultor process elaboration below).

An in-depth discussion on the presenting issue takes place between the involved parties to improve the consultee's understanding of the issue faced.

Other parties are notified that the issue arose, that understanding/resolution was reached and/or that information was sent to the consultee.

Table 12: Integrated consultee process diagram elaboration 


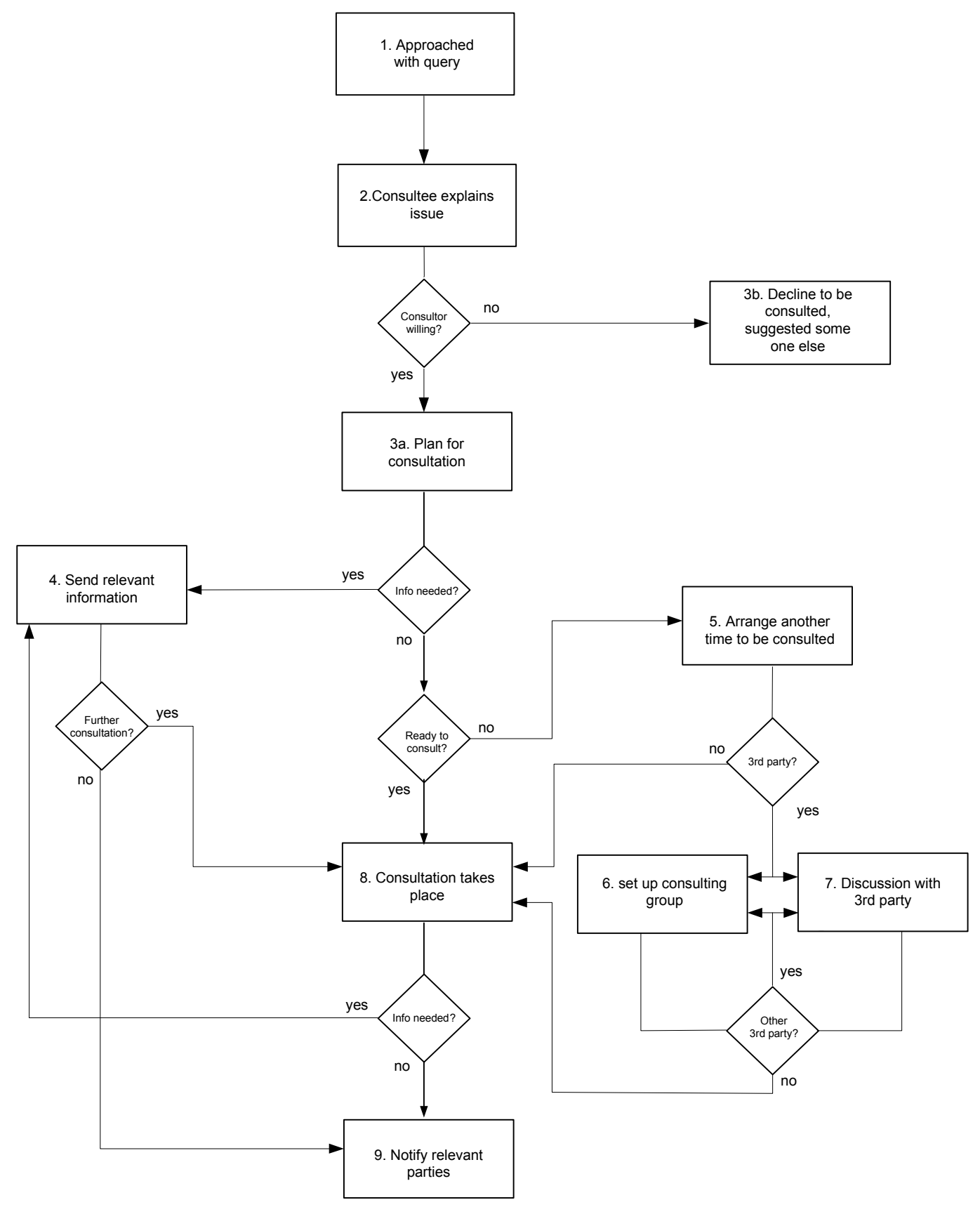

Figure 10: Integrated consultor process diagram 
1: Approached with query

2: Consultee explains issue

3a: Plan for consultation

3b: Decline to be consulted, suggest some one else

4: Send relevant information

\section{5: Arrange another time to be consulted \\ 6: Set up consulting group}

7: Discussion with $3^{\text {rd }}$ party

\section{8: Consultation takes place}

9: Notify relevant parties
The consultor is approached by a consultee with a query on a presenting issue.

The consultee will briefly describe and clarify the presenting issue(s).

The consultor agrees that they have the expertise and time necessary to consult on the particular issue brought to them and agrees to be consulted.

The consultor does not have the necessary expertise and/or time to be consulted on the issue, and declines to be consulted while suggesting another consultor.

If the initial issue appears to be a simple information request, or through consultation additional information is needed, the consultor gives or sends information to the consultee.

The consultor agrees that they have the necessary expertise to consult on the issue but the consultation cannot take place immediately, and so another time is arranged.

If the consultor finds that additional people need to be involved in the consultation then they will act to set up a consulting group.

The consultor pauses in the consultation in order to discuss the issue with another person or persons. The consultor may need confirmation of their intended response to the consultee, or they may not know the correct response at all.

An in-depth discussion on the issue takes place, including the consultee, consultor and any other required persons. The goal here is not to offer 'solutions' to the consultee, but improve the understanding of the issues faced though discussion.

Other parties are notified that the issue arose, that understanding/resolution was reached and/or that information was sent to the consultee.

Table 13: Integrated consultor process diagram elaboration

I presented the main findings of peer consulting practices to key participants (SMa and $\mathrm{SMb}$ ). Most importantly, I presented and described the integrated peer consulting consultee and consultor diagrams (see Appendix I for additional points related to the process diagrams), and received positive feedback: 
"Seeing the flow charts that you put up just made me realise how complex really, the communication process is even for a reasonably simple communication transaction. And it does feel complex to implement at times too. Particularly in the area of setting up face-toface dialogues with people who are often in sessions. So, this sort of highlighted that experience for me. And around the organisation" (SMa)

$\mathrm{SMa}$ and $\mathrm{SMb}$ formally signed off on each process diagram, and gave further comments through informal follow up discussions. Finally, I prepared a business report summarising key findings from the activities of developing the online peer consulting concept and mapping current peer consulting activities. This report was distributed to SMa and $\mathrm{SMb}$, and those participants interviewed as part of these Defining stage activities. Limited feedback from participants was received, and was used to inform subsequent activities.

\subsubsection{Documenting current ICT support}

This is the final activity of the Defining stage, within which I worked with CI to analyse the peer consulting process mappings (see Figs. 9 and 10) and assess which technology and systems tools were available and used within CI to support peer consulting activities. Data from interviews with Managers combined with my observations and notes from informal conversations with participants were used to form the basis of this assessment. When recounting peer consulting episodes participants described the types of ICTs they used to support their interactions. Through analysis, a number of key themes emerged as prominent across participant experiences, as summarised in Table 14. 
The nature and urgency of an issue faced

\section{Issue Urgency \\ Affected \\ Communication \\ Mode}

\section{Complexities of Communication Modes}

\section{Teleconferencing Preferred for Consultation}

\section{Internet (and intranet) Excluded from Selection} influenced the mode of communication used. Simple forms of ICT were used for making initial contact, holding a discussion (synchronous and asynchronous) and the exchange of information between peers.

Complexity is inherent in the use of each communication mode. With faxes, it is difficult to manipulate text for later use. Part-time positions, differing work hours and work-related travel resulted in unsuccessful attempts to contact peers (e.g. "telephone tag"), typically over three seven days.

At the stage of 'actual consultation' on the issue(s) (steps 9 and 8 of Figs. 6.2 and 6.3 respectively), teleconferencing was preferred when there were more than two parties involved. Examples of when this "consultation tool" was used are discussing upcoming training opportunities and gaining clarity around policy issues.

At the time of the Defining stage, the peer consulting process was not supported by Internet or intranet technologies (apart from the limited role of email) ${ }^{13}$
1. face-to-face

2. telephone

3. email

4. teleconference

5. $\operatorname{fax}$

2. telephone

3. email

1. teleconference

2. phone

3. face-to-face

Table 14: ICT tools used to support peer consulting at CI

Expanding on the Complexities of Communication Modes, many regional practice leaders elaborated on their experiences of attempting to seek and/or give help and associated resources to peers. Communicating via email was often problematic, again due to the part-time nature of positions, different work hours and the need for travel. In addition to these factors, some members of CI did not have individual/personal CI email addresses. In such a situation an email would go to the general office address

\footnotetext{
${ }^{12}$ Numbered from most to least used/occurring

${ }^{13}$ This is discussed in detail in the Structuring stage description (see section 6.2.2) where participants and I collaborated to determine technology choices to support the CI online peer consulting programme.
} 
before being forwarded to the intended recipient. This is viewed as being less secure and not preferable as a third party is then relied on to communicate information to the relevant person. An example the problematic nature of this situation was provided: An email message along with an attached document was sent to a participant who does not have an individual email address. The person received the document(s), but not the actual message that held the instructions on what to do with the document, and an indication of the urgency of the request. Although the person was grateful to receive the information, for some time they remained unaware that any action was required of them, which resulted in delays in the resolution of the particular issue. In addition, many of CI's regional practice leaders were not in the habit of checking their emails regularly, so many urgent matters were followed up with phone calls, or the use of email omitted altogether.

The key themes presented in Table 14 significantly influenced the types of ICTs that members of CI used to support their peer consulting interactions. These themes are consistent with issues identified in the discussion of peer consulting practices at CI (as described in section 6.1.2, above) and reflect the general complexities of their practice-related communication.

\subsubsection{Defining stage summary}

Members of CI began to transition towards online peer consulting by working through the activities of the Defining stage in order to further refine their online peer consulting concept. Specifically, investigation revealed that Managers typically faced regional isolation and worked with localised knowledge, confounded by the dispersed structure of CI and the nature of the Manager role. The ad hoc, informal and infrequent peer consulting that did take place was among peers with longstanding professional and/or personal relationships on a one-to-one basis.

$\mathrm{SMa}$ and $\mathrm{SMb}$ determined a strong need to improve connectedness of the participant group, and compliment face-to-face professional development activities with an 
online programme. Key members of CI and I collaborated to condense findings of this first stage into a statement of the desired outcomes for CI, or their Target for Change (Baskerville \& Wood-Harper, 1996) whereby Managers:

- can connect up to discuss practice related issues in between face-to-face meetings, at a convenient time and day

- establish a culture of sharing between peers, across regions

- compliment the one-to-one basis on which a consultation is currently based with one-to-many and/or many-to-many perspectives

- view use of online tools to seek and give help to peers as a usual work practice, part of their every day work

- experience a stronger sense of sociability and community within the peer group

Determining the current state of peer consulting at CI and generating the Target for Change made the potential for online peer consulting clearer, and resulted in key members of CI gaining new enthusiasm for the general concept and moving forward with the initiative. This is termed the Galvanising Point, and it is here that CI suspended their exploration of current activities of Managers, and turned to consider process and ICT elements of operationalising their online peer consulting concept.

\subsection{The Structuring Stage}

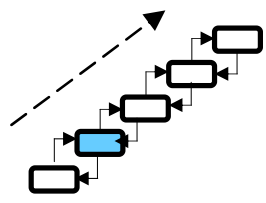

The Structuring stage at CI began with a review of the Defining stage outcomes, specifically the findings related to the current peer consulting activities, use of ICT by participants, and the Target for Change. Following on from this, SMa, SMb and I participated in a series of meetings though which we collaboratively determined the set of action-oriented change activities needed to achieve the Target for Change. Activities build on each other to form one cohesive Approach to Change (Baskerville \& Wood-Harper, 1996):

- Development of a plan for a series of online peer consulting sessions

- Selection and setting up of the Blackboard and Chatterbox systems

- Introduction of these systems into the participant organisations

- Training sessions and initial familiarisation period of systems use

- Use of the Blackboard system for ad hoc peer consulting 
- Use of the Blackboard system for a series of structured peer consulting sessions

Activities 1-4 occurred prior to formal online peer consulting sessions and are described here as part of the Structuring stage. Ad hoc peer consulting of Activity 5 is explained as part of the Experimenting stage discussion (section 6.3) and the action-taking of Activity 6 is detailed within the Refining stage (section 6.4).

CI developed an initial structure for online peer consulting activities by working through several key activities. This process structure was formed through developing a roadmap for a series of peer consulting sessions, including how they were to be supported technically and the capability of participants to engage. As CI determined such operational elements of their programme, I observed their key actions and organised them as the three activities of:

- Planning for a series of peer consulting sessions (Action 1 of the Approach to Change)

- Selecting and setting up systems (Action 2 of the Approach to Change)

- Training on systems use (Actions $3 \& 4$ of the Approach to Change)

The collaborative actions of research participants and I within each Structuring stage activity are described throughout section 6.2.

\subsubsection{Planning for a series of peer consulting sessions}

CI is an established organisation with some well defined professional development practices ${ }^{14}$. Therefore, we deemed it likely that a series of well defined and structured peer consulting sessions would be more successful than introducing opportunities for loosely structured, or ad hoc online peer consulting. When developing the plan we discussed issues such as: how to inform participants of forthcoming sessions, time frames, themes, resources, outputs and how/when to obtain participant feedback. This culminated in a session structure document that outlined an initial plan for a series of

\footnotetext{
${ }^{14}$ Refer to section 3.4.2 for a detailed discussion of this organisation.
} 
online peer consulting sessions. It was distributed to all CI participants for feedback and subsequent amendments were made (see Appendix J).

The document formed an important part of the Structuring stage, as including participants in the planning process raised the profile of online peer consulting at CI, set initial expectations of the participant group and also encouraged CI to develop a sense of ownership of the initiative. Additionally, discussions held while generating this plan helped to clarify actions required for achieving the Target for Change at CI, and how such actions fit with current, day-to-day work at another level of detail.

\subsubsection{Selecting and setting up systems}

The next activity we undertook was assessing the kind of technical support required. Selecting and setting up systems encompassed theoretical and practical elements, both of which are described below. Firstly, the actions of participants and myself that led to systems selection are described, followed by the systems set up phase.

\section{Systems selection}

The first step in assessing systems support for online peer consulting was to thoroughly consider the types of issues participants faced that would prompt them to consult a peer. CI Managers are tasked with continually assisting colleagues with a wide range of issues and problem situations. Within situations requiring peer consultation previously discussed (see section 6.1.1, above and Appendix F) some common factors were present:

- The involvement of multiple persons in each situation

- The part time nature of many CI roles

- The degree of urgency in resolving the situation or at least improving each parties understanding of the situation

- The geographical distance that separates peers

- The sensitive nature of the information involved in the situations 
CI staff faced issues such as those described above in situations with a relatively high level of complexity and equivocality as each party involved in a situation has access to different resources, including information and experiences. This can lead to different interpretations of the presenting problem or issue and associated information. $^{15}$

I then turned to the peer consulting process, to identify points at which ICT could most effectively support online peer consulting, and the types of media/ systems functions required. I applied MST to specify the technology choices most appropriate in supporting peer consulting at CI. I identified the prominent communications processes within each stage of the integrated consultee and integrated consultor process diagrams, which are presented below (in Figs. 11 and 12), followed by an explanation of the identified communications processes.

Stages 4 and 8 of the consultee diagram (below) show no prominent communications process. As the consultee is not interacting with another party during these stages, no communications process was assigned.

\footnotetext{
${ }^{15}$ See Chapter 5, section 5.2.2 for a detailed discussion of complexity and equivocality, and their impacts.
} 


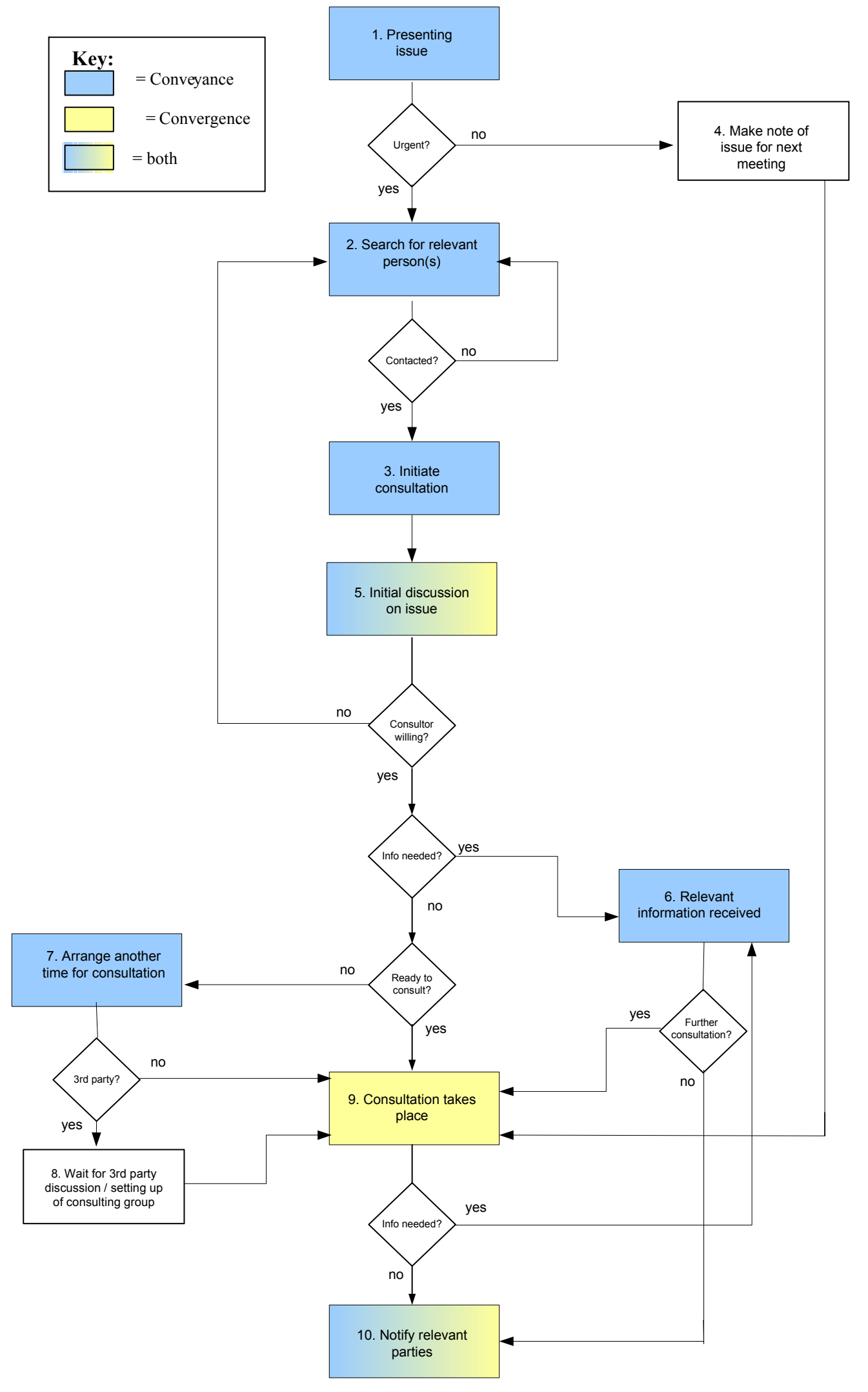

Figure 11: Integrated Consultee Process Diagram Showing Communications Processes 


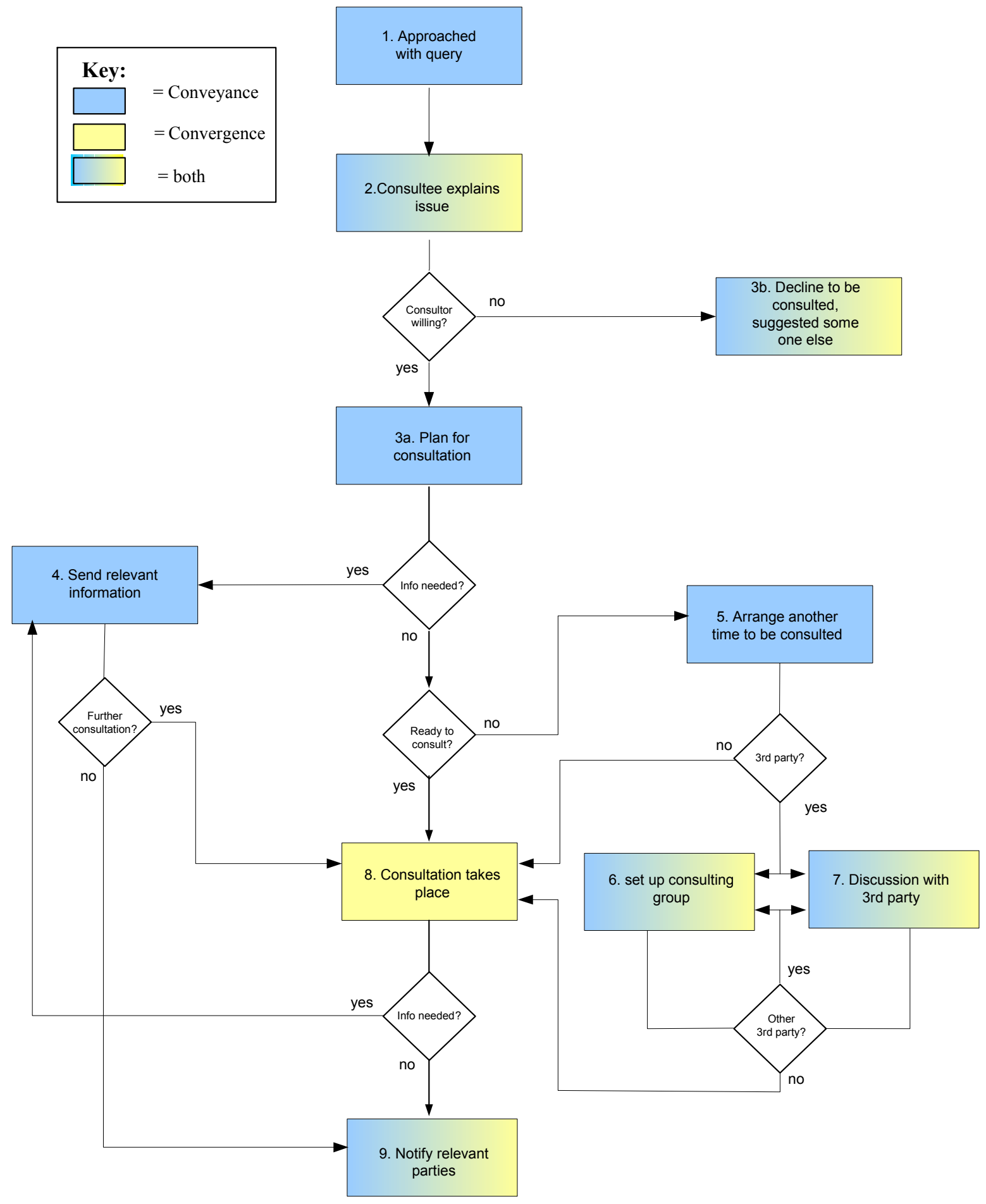

Figure 12: Integrated Consultor Process Diagram Showing Communications Processes 
As evident in Figures 11 and 12, many steps of both the consultee and consultor perspective of the peer consulting process are predominantly conveyance, defined as the dissemination of a variety of previously known information (refer Chapter 5, section 5.2.2). Here, information exchange occurs whether through relaying factual information in a conversation or dissemination of documents or images (Dennis et al., 2008). When seeking advice from a peer, it is usually without essential knowledge or information that they require. Also, at different times within the consultation process Managers will need to obtain any knowledge or information that they require to continue a consultation.

Some stages are shown as involving conveyance and convergence, for example stage 5 of the consultee process and stage 2 of the consultor process (in Figs. 11 and 12 respectively). This is because as the consultee begins to explain an issue to the consultor in a factual sense (describing key aspects of the situation), conveyance is the prominent communications process. However, in this same stage the consultor may respond with brief clarifying questions or comments and some convergence may then occur as a shared understanding of each others interpretation of the factual information recounted may take place. The prominence of either conveyance or convergence within many steps of the peer consulting process will therefore depend on the nature of a particular consultation, especially the issue at hand and the information accessible to each party.

Within the stage of peer consulting where the actual consultation takes place (stage 9 of the consultee process and stage 8 of the consultor process) peers attempt to understand a problem situation (such as those listed above) which requires them to agree on a shared meaning of the information, from which appropriate actions can then be based. This is prominently convergence, described by Dennis and Valacich (2008) as the process by which a group compares their conclusions on an information set and reaches a common understanding, in the context of their current situation (refer Chapter 5, section 5.2.2). 
As illustrated by Figures 11 and 12 and discussed here, the peer consulting process at $\mathrm{CI}$ includes a mix of conveyance and convergence, and a mix of low and high media synchronicity is needed in support. As described by Dennis \& Valacich (1999), conveyancing requires a communications environment that supports low immediacy of feedback and high parallelism (the number of concurrent communications that can occur), defined as low media synchronicity. Convergence requires high immediacy of feedback and low parallelism, defined as high media synchronicity and the media that is best matched to one communications process is often not ideal for the other. Some media choices appropriate firstly to conveyance and then to convergence within the peer consulting process at CI were ${ }^{16}$ :

- Conveyance:

- Written and electronic mail

- Asynchronous groupware (threaded discussions)

- Synchronous groupware (online chat, text based)

For the conveyancing required within early stages of the consultee and consultor perspectives of the peer consulting process, simple email capabilities were deemed to suffice.

In addition to this, before or during the consultation stage of the process the exchange of needed information or documentation could occur via email attachment. Threaded discussion forums and online chat facilities could also be used for requesting needed information, although at the time of this activity of the Structuring stage it was dependent on their configuration as to whether these technologies could be used for the actual exchange of information.

- Convergence:

- Face to face

- Telephone

- Tele/Video conference

- Asynchronous groupware (threaded discussions)

- Synchronous groupware (online chat, text and voice based)

\footnotetext{
${ }^{16}$ See Chapter 5, section 5.2 for a discussion of how these media satisfy the high/low synchronicity requirements, as outlined by Media Synchronicity Theory (Dennis \& Valacich, 1999).
} 
Although face-to-face and telephone interactions are somewhat rudimentary, traditional communication channels they are important mediums for peer consulting within CI, as described above, and thus were not abandoned. In addition to these, increased use of teleconferencing and the introduction of text and/or voice discussion boards and online chat facilities were prescribed to help overcome the problems of geographical isolation, part-time work hours and the need for travel that disrupt their peer consultation process. Participants can then change between communication media as their needs change, known as media switching (Grandhi et al., 2003).

Teleconferencing and online chat fulfil the high feedback and low parallelism requirement of high media synchronicity. These technologies allow parties to a consultation to give rapid feedback on a communication that they receive, and support the bi-directional communication discussed by Dennis and Valacich (1999) that is essential during the stage when the actual consultation event occurs. Dependent on configuration of a particular medium, with teleconferencing and asynchronous and synchronous groupware it is possible that only one conversation can exist at any one time, resulting in low parallelism.

Although these technologies have the characteristic of low parallelism, they do support the one-to-one, one-to-many, and many-to-many requirement of peer consultation within $\mathrm{CI}$; one main addition to the mapping of the peer consulting process is the introduction of the one-to-many and many-to-many perspectives. With these perspectives, a group of peers can consult on an issue, as opposed to only one consultee engaging with one consultor. Tele/Video conferencing, online chat and other web-based audio conferencing tools can support small groups of peers meeting together in real-time to discuss a problem situation. Threaded discussion forums are listed here as a complementary medium to teleconferencing and online chat. After a synchronous consultation session, discussions that improve understandings of a particular problem situation, issue, or information could be continued via threaded discussions, thus leading to increased convergence. 
In summary, the application of MST revealed that the peer consulting process within CI involved a mix of conveyance and convergence, and both communications processes had to be supported for a successful consultation to take place. Following this, we developed a set of criteria against which candidate systems could be assessed. These criteria included the findings detailed above combined with additional factors that research participants identified:

- Expense of the system,

- Availability of the system,

- Complexity of the system,

- Technical skills of participants,

- Technical support needed and available,

- Hosting options.

After canvassing potential systems I presented a number of systems options that best met the selection criteria, including Microsoft Sharepoint, Chatterbox and Blackboard. To communicate the potential of each system I showed examples of systems, and where possible orchestrated live demonstrations.

During the next phase of systems selection we assessed each system in turn. More specifically, how the functions of a particular system could support the communication processes within each distinct stage of the peer consulting process. Two complementary systems of Blackboard and Chatterbox were deemed most appropriate for use as the pilot online systems (see Table 15). A view of the Blackboard window is provided in Figure 13 below. 


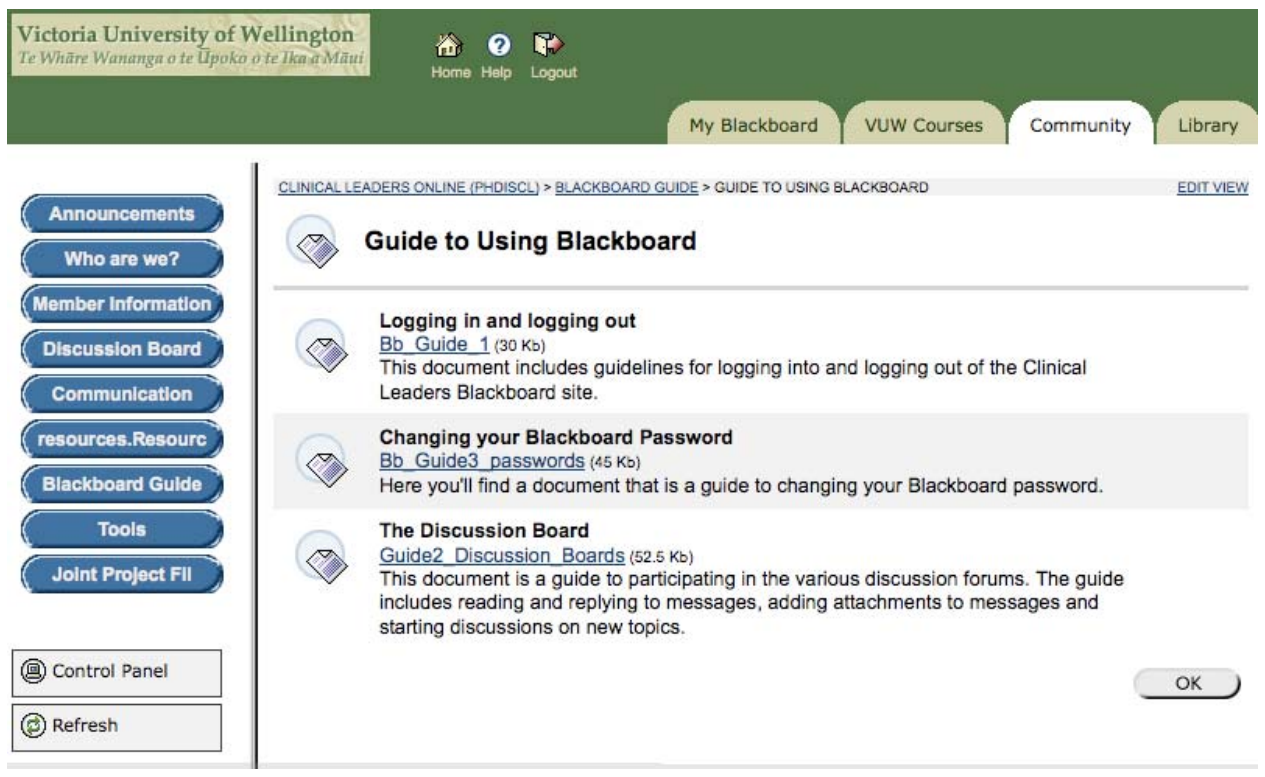

Figure 13: View of a Blackboard Window

Additional criteria, as previously listed, were also satisfied by the selection of

Blackboard and Chatterbox:

- Both systems were at no cost to CI

- Systems were available after a short one month set up period

- Systems were relatively simple to use

- After a basic training session the technical skills of participants would be adequate to use these systems

- Technical support needed was minimal, and could be offered by myself and University technical staff

- The University would host both systems

As these systems were intended to complement, not replace, existing peer consulting channels, use of email, conference calls and face-to-face meetings would continue. 
- Document storage and exchange

- Asynchronous text-based discussions

- Announcements posting

- Group member profiles

- Further member information

"I think threaded discussions are the things that would be of most interest really. That thing about people who are interested in a given topic, being able to have that conversation at a time that is convenient to them. So they don't have to make it at a particular time because timing is always a huge

- Additional, more advanced features issue." (SMb)

Media Synchronicity Theory: Blackboard supports conveyance and (to a lesser extent) convergence.

- Synchronous voice based discussions

- Simple synchronous text based discussion

- Displaying presentations (eg MS PowerPoint)

- Web browsing

"For our people I think something that has that voice component will be really good. Some of our people are more used to email and making that more fun and personal, but our work is very much around talking, and conversations. So getting this around people's voice is really helpful, I think for those people to engage. Also, there'll be a bunch of our folk who are not so quick on the keyboard, and so talking is a lot easier. So I think that will be great." (SMa)

Media Synchronicity Theory: Chatterbox supports the communications process of convergence.

Table 15: Attributes of selected systems

\section{Systems Setup}

Once systems had been selected we moved to readying the systems for initial use by

CI. We named the site Regional Practice Managers Online and I then made key areas of the site live, including:

- Announcements

- Group and leader information

- Discussion boards

- Content areas

Participants provided content to populate to site, and offered feedback on the organisation of site elements, which informed later changes. 
Finally, we tested the functionality of the system, and at this time SMa and SMb also commented on the general 'look and feel' of the site, which informed further changes. Additional changes are described within the Experimenting and Refining stages, below (Sections 6.3 and 6.4 respectively).

When we agreed that the Regional Practice Managers Online site was ready to be launched, initial systems set up ceased and the activity of developing and delivering systems training was actioned, as next described.

\subsubsection{Training on systems use}

As previously discussed, most members of the participant group had limited experience and knowledge related to online systems and technology. It was therefore vital that they developed the capability to participate in online peer consulting through training. One participant commented:

"I think that the other challenge is around... training and accessibility to the level of computer literacy that we've got in our [participants]. We've got some groups that are really computer literate and then we've got some groups who are still struggling to use email. And so what we might consider, or what I might consider to be a reasonably simple technology to use, may bring out anxieties that are in certain people." (SMa)

Based on such suggestions, we developed a short training session that I delivered in a one-to-one format ${ }^{17}$ during a CI national management meeting. Because of their individual busy schedules it was more convenient and necessary to arrange individual training and coaching on a one-to-one basis. Through 15 minute sessions, I introduced participants to the site, and the essential functions of the systems such as finding, accessing and navigating the system. Participants reported mixed experiences

\footnotetext{
${ }^{17}$ Within the context of this study the one-to-one format implies the researcher's individual interactions within each participant.
} 
training sessions, particularly the placement of the sessions within the wider management meeting. This is evidenced in the following comments:

"For me it was very rushed and squeezed in between different workshops that I was getting to at [the national meeting]. In the context of the meeting it was really hard to find the time to put in there" (P4)

and:

"I think that the way that it was delivered was that even I could pick up what it was about. I am not particularly computer literate, so I think that part of it was excellent. I'm not sure it was a good forum though, at that place because there was so much going on and our heads were elsewhere." (P8)

To complement training sessions I developed written guides to using the various areas of the site. Participants experienced with using online systems reported that they did not need to refer to the written guides, while others group found value in the combination of the practical and written training material, as reflected this typical comment:

"Because you did a verbal explanation and you also give us a written explanation, which I found really helpful to have both... I left the introductory session pleased to have some written material that I could go back to and refer to when I got home because it was a step-by-step approach." (P3)

Each Manager present at the national management meeting participated in a training session. As changes in the wider participant group occurred I conducted introductory training sessions for new staff via telephone and sent written guides out via email.

\subsubsection{Structuring stage summary}

Applying the knowledge gained at the Defining stage, I worked with members of CI to develop an initial blueprint for introducing and carrying out online peer consulting, collated into CI's Approach to Change (see section 6.2.1 above). Through Structuring stage activities we addressed four aspects of this Approach. A central 
component was producing a detailed plan for a series of sessions (see Appendix J) that highlighted important tasks, resources and processes leading up to, during and in between sessions.

Combining the plan, knowledge of CI, the participants and their capabilities, and findings from applying MST the Blackboard and Chatterbox systems were chosen to support online peer consulting at CI and subsequently prepared for use. Systems training increased the readiness of participants to engage in sessions, and generally improved their understanding of the nature of online peer consulting. CI had reached the Readying Point, and ceased Structuring stage activities as participants were prepared to put their newly developed capabilities into action to make initial online connections with peers in the Experimenting stage.

\subsection{The Experimenting Stage}

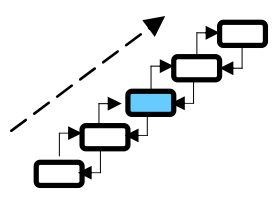

Building on the key outcomes of the Defining and Structuring stages, in this third stage of development CI actioned their online peer consulting concept. Primarily, they put their newly developed and/or extended capabilities into practice, complementing their use of conference calls and email with using Regional Practice Managers Online site for ad hoc knowledge exchange, an informal peer consulting activity.

Through connecting with peers online and gaining experience with functions of the site CI further developed their online peer consulting concept for the participant group and the wider organisation. As systems use continued throughout the Experimenting stage, CI amended their plan for structured sessions and also the configuration of the site. I present these core Experimenting stage activities at CI as:

- Actioning informal online peer consulting

- Restructuring sessions

These are discussed within the remainder of section 6.3 . 


\subsubsection{Actioning informal online peer consulting}

The Experimenting stage began when key participants and I met to discuss what subactivities would combine to make up the informal online peer consulting period. Key participants voiced concern at the mention of ad hoc peer consulting whereby Managers independently chose when to participate and what topics to base their discussions on. Therefore they put forward their view of an informal, but loosely structured online peer consulting period:

"I'd probably tend to build in a bit more structure, so that you've got all of this structure so that they use it regularly for the rest of the month, instead of leaving to up to people. Because if you leave it up to people it won't happen. Generally. The [Managers] are busy, it's a new thing, if they don't quite know how to do it... you know. It might be better actually to assert a structure whereby they are using it for a period of time. And then after that, it's up to them." (SMa)

As a result, we introduced structure that focused topic-based discussions across a particular time period. The duration of this period was expanded from four weeks to three months in order to allow enough time for the least experienced participants to become comfortable using the site, as SMb suggested:

"I'd think probably two or three months. Given that there'd be quite a lot of people who'd stuff around quite a long time before they'd get in there. And because we'd need to allow for some time for word to filter through the organisation that people were using it and liking it." (SMb)

Following this initial structuring of the informal sessions, we explored how the online system could best support early knowledge exchange at CI. We identified the recent national mangers meeting as an event that participants had some shared experience of, and where they had participated in training sessions. In order to enhance and leverage on earlier shared experiences I set up a Meeting Follow Up discussion forum to support their continued conversations on issues raised at the meeting (Akkerman et al., 2008). Some of the first participants to access and use this forum were those that had more previous experience with online systems, although those who had less 
experience also participated and were mostly enthusiastic, P6 exclaiming: "Hey I'm in here and guess what it is easy!"

However others struggled initially with use of discussion board functions, as P9 expressed, and peers often assisted them (with some humour):

"Is it just me but some of the buttons I push (on the website of course) seem to only work occasionally? The discussion board, resources tabs hardly ever work? (It could be me as my skill on the computer is legendary)?????????” (P9)

"I think you have been overdoing it [P9] and it isn't responding! :) (P17)

Other participants began to interact on the Meeting Follow Up forum, and held a discussion centred on issues of online communication etiquette. This showed that they were developing a deeper understanding of the process of online peer consulting, in particular their use of the online system for discussing issues of practice. P12 offers a typical comment:

"I am thinking that this place of communication does need some discussion of etiquette/ethics. It seems to me to be not very private. I have had an experience of an online classroom having a technical collapse, and all the contents were all over the floor. I am also aware of how I speak in shorthand when I am here at the keyboard, and what I say could be easily misunderstood. I think what I need is an agreement among us that we will ask for clarification when the meaning is unclear or ambiguous or has the potential to skew our relationships.

What do you all think?” (P12)

This discussion considered many issues, including:

- Ethical considerations

- Potential for misunderstandings

- Need for confidentiality

- Remaining non-judgemental

- Openness

- Accountability 
Once the discussion on issues of etiquette came to its natural end, we determined that a new discussion forum was required to focus discussions firmly on issues of practice. We labelled the new forum Hot Topics, where participants could raise and discuss urgent and topical issues of practice. To encourage discussions in the new forum, I worked with key participants and identified topics and issues raised in other forums that could spark discussion in Hot Topics. I invited those who authored the original messages to start a new discussion, which resulted in four new discussions on the Hot Topics forum.

As participant use of the forums continued, many switched between forums, specifically the Training, Meeting Follow Up and Hot Topics forums. While some participants adapted to participating in multiple forms concurrently, others found the ongoing discussions across three separate forums difficult to follow:

"I'm wondering if time has passed since the [National] meeting and whether we should concentrate any discussions now in the [Hot Topics] Discussion Forum place. This would mean only having to check in one place. So, I will go there now." (P17)

In response, I archived the messages posted to the earlier forums and deactivated them so that messages were read only. As intended, discussions were then focussed in the Hot Topics forum, which remained lively throughout and after the Experimenting stage (see the Refining stage, section 6.4 for additional discussion).

At the conclusion of the three-month period of actioning informal online peer consulting, 15 Managers had posted a total of 62 messages across three discussion forums. They maintained a variety of collaborative discussions, some participants switching between forums and maintaining multiple discussions concurrently. Those who actively participated in these discussions experimented with systems use and became familiar with the basic functions of the site, as intended. They developed competencies such as:

- Accessing the site and discussion forums

- Reading messages and posting responses 
- Starting new discussion threads

- Problem solving technical (and other) issues

Participants began to build an individual and collective reservoir of experience around the use of the site for peer consulting, both evolving their understanding of the online peer consulting concept, and increasing their capability to participate in the formal online peer consulting sessions of the Refining stage.

\subsubsection{Restructuring sessions}

Once the period of informal consulting ended, we discussed the nature of participant interactions and their use of the discussion forums and other site functions. I analysed data including site statistics, discussion board posts, and participant testimonials from which three prominent issues emerged:

- Prioritization

- Privacy

- Communications format

Each issue is discussed as part of restructuring CI's plan for formal peer consulting sessions (see Structuring stage, section 6.2.1).

\section{Prioritization}

CI is structured regionally, typically with one Manager responsible for overseeing the staff and clients of one region. The regional service provision focus introduces significant time constraints; their time was devoted to the day-to-day issues of supervising and supporting staff and delivering services to clients. Therefore, informal consulting (and likely also formal sessions) was not prioritised by many participants, who were unable and/or unwilling to spend time learning to use, or using a collaborative technology such as online discussion forums for peer consulting. Participation in such activities was perceived to be at the expense of their core work, as illustrated: 
"If there's a lot of pressure on, the participation might reduce. Yes, and that depends... I would have to say that given client and counsellor needs, I would certainly put them higher up on the priority list than Blackboard" (P2).

In contrast, others stated that participation did not need to take a lot of time, due in part to the asynchronous format of discussion forums:

"I would still really love to use Blackboard as a place to hold those kinds of discussions. I think its got great potential, I don't think it needs to take any more than a few minutes a day. Like, it's really quick, much quicker than a phone call. And I would like it because you can access it when you are ready, and not having to respond in the moment." (P12)

Some participants overcame such demands on their time during work hours by devoting personal time to learn and use the forums and other site functions, as P3 recalled: "I do all my computer work at home, because it is easier for me. I mean, I have got a computer that I could use at work. But I tend to work in the evenings at the computer."

The competing demands for Managers time affected the nature of their online interactions, as many logged into the system infrequently, or for short periods of time to read the most recent posts and/or post brief messages. Those who logged in more frequently, and posted a higher number of messages during the Experimenting period became discouraged:

"I was really keen and started a couple of threads and joined in threads until I got embarrassed about my over presence... Because at one stage I was fifty percent of the input! And then when we had a regional teleconference on it everybody was saying they didn't have time to do it. I kind of got embarrassed by that again too." (P12)

$\mathrm{SMa}, \mathrm{SMb}$ and I discussed the prioritisation of online activities with other demands and decided that the original plan for the series of formal online peer consulting 
sessions should be amended to incorporate a longer time period for each session, and also a greater amount of time between sessions. It was anticipated that widening the time periods slightly would encourage participation and make it easier to balance the demands of their workload with participating in online peer consulting.

\section{Privacy}

"It's like a conference call, with everyone listening, though they may not be saying anything" (P15)

When interacting online, many participants were influenced by their perception of the discussion forums as a public domain. "A consistent finding reported in online privacy research is that an overwhelming majority of people are 'concerned' about their privacy when they use the Internet" (Paine, Reips, Stieger, Joinson and Buchanan, 2007; p.526). Likewise, the privacy and security of the forums was discussed by CI throughout the life of the project indicating the importance of the issue. A comment of P12 typifies this:

"I think whenever I have conversations by email or on a place like Blackboard I have an expectation that that material is accessible... like, if there's a computer break down then 'whoops', people get to read it." (P12)

Issues of privacy and security and a general lack of confidence in the technology of the Regional Practice Managers Online site influenced the topics discussed, and also the additional time that participants spent preparing and posting messages. Taking such factors into consideration, we amended the list of topics appropriate for discussion during formal sessions of the Refining stage, and felt the earlier decision to lengthen the time period for each session and between sessions had been validated.

\section{Communications format}

Combining with the prioritization and privacy issues, the asynchronous text-based format of the online system and discussion boards and forums of the Experimenting stage also influenced participants peer consulting interactions. P2 explains such issues: 
"...well, there are a number of them really. One is immediacy. It's very different from a conversation where you say something and you get an immediate response. I guess the next one would be that there are...well, you called them social cues but its body language, tone of voice, that kind of thing, which isn't present. And so that can leave things open to misinterpretation. And the other thing is, is that it's more effortful really. Because when you talk, things just come out of your head. But it's kind of more purposeful or more thoughtful when I sit down and construct a response to a question." (P2)

This statement highlights some important issues. Firstly, the ICTs introduced another kind of social and psychological distance between participants (Kehrwald, 2008), providing a very different interaction experience. This notion of distance is a key element of the dynamic, relational view of social presence, which argues that the degree to which individuals involved in a communicative exchange are able to perceive the presence of others is typically as important as aspects of the media (ICT) itself (Kehrwald, 2008).

Secondly, participants highlighted concerns centred on the apparent 'permanency' of online content, also termed 'reprocessability' (Dennis et al., 2008) and that without mechanisms to check that their messages were interpreted as they intended, such messages were open to misinterpretations. Thus participants more carefully considered the content and tone of their messages than if communicating in an environment that allowed for greater immediacy of feedback (Dennis et al., 2008). Thirdly, text-based communication on issues of practice took considerable additional time and effort (due in part to higher reprocessability), as compared to a face-to-face or telephone situation.

Participants reported that they were often apprehensive about contributing to a discussion if they were unsure whether it was active or not, or perhaps moved to another forum. Thus, the views of some participants were not represented at the Experimenting stage, due to the asynchronous and text-based nature of the forums, as P4 summarises: 
"The written medium of [the forums] means that sometimes you may think that... you're not quite sure when a topic has ended. You know, in a face-to-face or over the phone, there's an ending to a topic and in that medium of everybody coming in and posting on it's kind if like, well, some body else may have moved on, and it still is carry on with another life." (P4)

In contrast, other participants recognised the positive impacts of the asynchronous, text based communications format, for example:

"...Because our timetables are always hard to work around and through. In particular, I mean just making contact with people here in the office can be tricky. But the advantage for Blackboard for that is that it's not in real time." (P12)

The asynchronous nature of the discussion forums enabled participants to contribute to conversations at a time convenient to them and creating an ongoing, threaded discussion that they could follow across time and contribute to more than once as their ideas developed. Based on participant experiences, the decision to increase the time frames of formal sessions, and the period between sessions was reinforced.

Based on the findings related to the issues of prioritisation, privacy and the nature of the communications format, we amended the structure for the series of formal sessions to improve the likelihood that participants would engage in, and receive value from, the Refining stage sessions as next discussed.

\section{Altering the plan}

Key changes focussed on the duration, topics and general approach to each session. The intended timeframe of each session was extended to two weeks and the time between sessions to three weeks to allow for session preparation and notification, the generation of outcomes and the assessment of the session (see Appendix J). We deemed this five week timeframe broad enough to allow time pressured participants to engage in sessions, without being too broad so as to break the 'momentum' of the series. 
Following this, in light of comments regarding the privacy of the system and the sensitivity of information posted to the forums, we reassessed the initial list of session topics. Instead of posting broad questions on issues of practice, we deemed it more appropriate to base session topics on participants' own experiences, related to current literature, and processes, policies and programmes within CI. Finally we altered the session notifications to be sent one week before and then a day before the commencement of each session, and session progress updates at appropriate times during each session. This proactive approach was intended to remind participants of sessions, and to interest them enough to potentially entice them to participate more fully in a session.

\section{Altering systems set up}

Changes made to the plan for the series of formal peer consulting sessions, as described above, required little change to the configuration of the Regional Practice Managers site. As Lock (2006) found, the redesign of a professional development process or components of that process require a re-think of the supporting environment, in this case the Regional Managers Online site. Changes included:

- Altering titles of discussion forums to reflect the new topics

- Altering the active duration of forums for each session

- Changing the set up of the discussion forum to include one main thread based around a posted session resource

Completing alterations to the session plan and the site concluded the activity of restructuring sessions.

\subsubsection{Experimenting stage summary}

CI participants gained experience of the online system through engaging in and/or following (through 'lurking') informal conversations with peers on the discussion forums of their online site. This complimented their existing use of email, phone and conference calls as peer consulting channels and their current media switching 
practices (Grandhi et al., 2003), and furthered their understanding of the potential value of online peer consulting. I deemed discussions at the Experimenting stage informal, due to the absence of:

- formal scheduling of sessions

- scheduled participation

- prepared discussion questions

- session/ period output

- a series of interlinked sessions

Although informal, these discussions were not ad hoc in nature as initially intended due to a finding of the Scoping and Structuring stages that revealed myriad demands on participants time, and therefore also the possibility that they may not engage in discussion and experiment with the online forums. As a result, the three-month time frame of actioning informal online peer consulting provided a loose structure that was reinforced by named forums that reflected initial discussion topics.

Participants with some prior experience and comfort using online tools and/or forums were first to engage in Experimenting stage discussions and connect with peers, some also participating in multiple concurrent discussions across different forums. Participants found that key issues of prioritization, privacy and the asynchronous, text-based format of the forums prevented them from engaging in informal peer consulting sooner and/ or more fully. In response, we made minor amendments to the plan for formal peer consulting sessions to be held at the Refining stage, reassessing session topics as well as allowing more time per session and between them. As they built individual and collective experience, I observed discussion topics changing naturally as interest shifted. Some discussions, such as 'online communication etiquette' demonstrated their growing understanding of the nature of online peer consulting.

During the Experimenting stage participants exchanged information about aspects of their practice including current issues, processes and policies, and the nature of their discussions evolved into collaborative knowledge exchange that constituted informal online peer consulting. This encouraged the continuing use of the forums and 
reaffirmed that technology has potential to support peer consulting within $\mathrm{CI}$ in a participant led, semi-structured manner. Through reaching such outcomes, CI illustrated that they had achieved a level of preparedness required to reach the Mobilising Point. Specifically, the participant group was familiar with online peer consulting and had developed their capability beyond Experimenting stage objectives. As their individual and collective knowledge and experiences gained at this stage motivated CI to advance their online peer consulting programme, they naturally moved through the Mobilising Point and to the next stage, Refining.

\subsection{The Refining Stage}

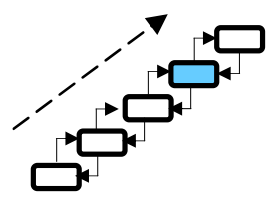

At the Refining stage, CI built on Experimenting stage outcomes with the objective of formalising online peer consulting by extending and refining the process and the supporting site. I encouraged members of CI to advance from ad hoc, unstructured peer consulting activities to participate in a more structured and formal online peer consulting session. As the session unfolded, I reformed the online peer consulting process and subsequently reconfigured aspects of the website. Our actions at the Refining stage are organised into three categories, represented as the key activities of:

- Performing formal online peer consulting

- Reforming online peer consulting

- Reconfiguring systems

Each activity is described below as a component of the Refining stage.

\subsubsection{Performing formal online peer consulting}

The peer consulting session plan developed during Structuring stage and then amended within the Experimenting stage (see section 6.2.2 and 6.3.2 above, and Appendix J) and with CI's growing repository of online peer consulting experience were the main inputs to this activity. 
As CI shifted to performing formal online peer consulting within Peer Consulting Session 1 we undertook a number of tasks, specifically:

- Preparing for the session

- Supporting participants as they engaged

- Assessing session outcomes

Each sub-activity and the particular collaborative actions CI and I moved through are detailed below.

\section{Preparing for Peer Consulting Session 1}

In preparing for the first formal session, we focused on determining the session topic and establishing the session. In determining the session topic we first assessed the types of issues that participants were most likely to gain value discussing. Referring to the list of appropriate topics elicited at the recent national CI meeting and feedback from participants obtained through conference calls, key participants and I determined the issues most suitable for online peer consultation. Factors that influenced issue selection included:

- Whether an issue was broad in nature and relevant to most of the participant group, as opposed to a specialist area

- The sensitivity of some issues (as many issues are more suited to face-toface discussion)

- What else was going on within the organisation, for example training in a particular practice area may make corresponding issues more topical

This resulted in the selection of the Session 1 topic and generation of a list of possible topics for subsequent peer consulting sessions (see Appendix J). Assessing and selecting topics led to a deeper understanding of the nature of the practice-related connection and interaction that CI desired through online peer consulting. As such, key participants and I more deeply understood the nature of the problem situation at CI (as initially outlined within the Defining stage). 
Establishing the session began with $\mathrm{SMb}$ exploring the session topic in more depth, and generating a summary of recent literature as a discussion document intended as the starting point for discussions, on which participants could reflect and comment. Next, I set up the discussion forum and posted an initial message along with the session resource. It was then a week prior to the session and time to notify participants, which I did via an email confirming the session date and duration and key resource, and encouraging participants to check their site logins and seek any needed assistance. The provision of additional training (via phone, and written guides) and technical support became an important Establishing activity, particularly for those who had recently joined CI.

Finally, one day prior to the session commencing I sent out reminder email, and again offered technical and general assistance to those who required it.

\section{Engaging in Peer Consulting Session 1}

The Establishing tasks laid the foundations of support for participants engaging in formal peer consulting. Specifically, the online forum provided a structure that supported their threaded, text-based discussion. Through this forum, CI posted messages reflecting on the discussion document, describing their practical experiences as well as posing questions for peers to consider and respond to.

After one week participation in the session was low, and I emailed participants informing them that there were several messages that they could review and comments on, the session remained active for another week, and inviting anyone to contact me if they required assistance with accessing and/or using the online system and session discussion forum. While the number of 'lurkers', or those Managers "seen but not heard" (Saint-Onge \& Wallace, 2003; p. 43), increased in response, with four additional participants viewing messages and tracking the conversation (discussed further below), there were no additional contributors to the session 1 discussion. 
After the two-week period of the session concluded, I 'closed' the session, by:

- Sending an email announcement

- Making the session forum unavailable (or inactive)

- Archiving the messages that were posted (leaving them available for viewing, but not amending or replying to)

The set of archived messages then became the output of Peer Consulting Session 1. This archive was termed an output, as it became a resource that was accessible to Managers beyond the conclusion of the session.

Despite the collaborative approach taken in preparing for the session and the initial enthusiasm from CI towards the peer consulting concept, only three of 14 participants engaged in the online discussion of Peer Consulting Session 1. They accessed the session forum, read messages and posted one reply each, resulting in three isolated messages. Additionally, P4 posted a message informing others they could not contribute to the session. On reflection, P4 commented:

"my holiday was definitely a factor in 'nope, I'm out of here' and coming back in... I think I posted a thing just saying you know, 'urgent priorities, I am not able to participate' (P4)

Although participants were aware of the session and demonstrated a willingness to participate, workload as well as other (external) commitments prevented some members of $\mathrm{CI}$ from participating, as $\mathrm{SMb}$ outlined:

"I think it's a little bit disappointing, that there was so few people decided to participate. But actually, when I think about the sort of circumstances that lots of people are in and it's not that surprising really... and there's quite a lot of upheaval, for quite a lot of people at the moment... within the role and their place in the organisation" (SMb)

Four participants gained some value from the discussion through 'lurking', whereby they accessed the session resources, viewed messages and tracked the conversation, 
but were not compelled to or could not contribute a message (Saint-Onge \& Wallace, 2003). P2 describers one such situation:

"Well, I attempted to [post a message]. I responded to it and I put a lot of time and effort into constructing something useful and maybe a bit challenging. And then I think I clicked on the 'send' or some thing and it went to heaven... and it lost itself... I can't remember the exact process but I know that I set aside some time to respond to the paper and I did it, and I spent a lot of time constructing a good response. And then I tried to submit it and it didn't submit and it was lost forever" (P2)

In summary, participation in the session was minimal, with three practice-related messages posted, one additional message and four other participants viewing messages but not contributing directly to the discussion. Therefore, the collaborative discourse that was generated within the Experimenting stage was not evident in Peer Consulting Session 1 of the Refining stage. On conclusion of the two-week session period we assessed the key factors that prevented CI participating in the session.

\section{Assessing session outcomes}

In assessing the outcomes of Peer Consulting Session $1 \mathrm{SMa}, \mathrm{SMb}$ and I reviewed data including:

- systems statistics

- discussion board posts

- my observation notes

- participant testimonials

From my analysis, several issues emerged as most prominent:

- managing and balancing priorities

- site and forum format

- technical difficulties

- relevance of the session topic

- changes in the participant group ${ }^{18}$

\footnotetext{
${ }^{18}$ The issues of prioritization and the communications format were also present, and discussed as part of the Experimenting stage description (section 6.3 above).
} 
When attempting to engage in the session, many participants experienced difficulties accessing and/or contributing to the online discussion forum. I assisted participants that had password problems (forgotten or expired passwords), mainly via email, and through conversation learned that they typically did not turn to the written training guide for help. Others quickly became frustrated, did not seek assistance and abandoned the session altogether, due partly to competing priorities and workload, as P3 recalled: "it felt like the very bottom of my priority list because I was really busy."

It was not only those new to online forums and consulting that faced difficulties of a technical nature. The general text-based format was an issue that arose at different stages, which SMb sums up: "I think [CI staff] are good listeners and good talkers, and I don't think they are necessarily good readers or writers. So, it's a different set of skills." (SMb). With a higher level of experience and knowledge related to online discussions and supporting online tools some participants compared their Peer Consulting Session 1 experience to others they had:

"I found having to click onto every body's messages individually a bit slower. At the time I was working online at Waikato University and we could see people's messages up on the same page all the time... You can see by just scrolling up the last three or four contributions and then you can just go up to the top of the page and see the previous six. So that's quite helpful without going in and out and in and out and in and out of every body's separate ones. That would've made it easier for me." (P3)

Such comments provided valuable information that we took into account when reviewing Session 2 options (see 6.4.2, below) and also when reconfiguring aspects of CI's peer consulting site (see 6.4.3, below). SMb put forth the idea that "we could encourage the [Managers] who are more interested or more confident with it to kind of buddy up with some of those who are less so. And then maybe do some phone conversations or something with them, to encourage them." Although this aligns well with CI's peer consulting concept and would extend the support available to participants, there was not time to develop the idea further and implement a 'buddy' system within the timeframe of my research project. 
Members of CI offered their views on possible session topics, questions and resources as part of the Structuring stage. As such, I did not anticipate that relevance of session topic would be one of the key reported reasons for non-participation. P4 described this as "relevance to what is actually going on in my life at that moment" and went on to state: "if I do [need to consult a peer] I prefer to pick up the phone and connect with them on what is relevant... I prefer to actually do that on a needs basis."

Similarly, new staff that joined CI during the project and also during Session 1 were still in the process of familiarising themselves with the organisation, it's culture and people, and thus did not prioritize engaging in the session:

"I initially didn't really do any thing with Blackboard because I wanted to kind of suss out that lie of the land to see how people used it, how they responded, what the kind of unspoken rules of this kind of communication behaviour were before I used it." (P2)

\section{Summary}

Despite the provision of practical and written training, the use of conference calls and email before and during the session, and the availability of the online discussion forum during the session, many participants did not participate in the session, or participated at a superficial level by posting one isolated message. These isolated messages did not form the collaborative discussion needed for peer consulting to occur and, as SMb suggested, participation may have been out of obligation to the research project, rather than driven by any expected benefit to be gained from participating in the session.

Final closure of Peer Consulting Session 1 came after a CI national meeting, where I facilitated a discussion on general session participation and experiences, and ideas for future online peer consulting. This concluded the activity of performing formal online peer consulting at CI. 


\title{
6.4.2 Reforming online peer consulting
}

Following Session 1, we turned our attention to continued online peer consulting at $\mathrm{CI}$, and how to evolve the structure of the series of sessions with the objective of providing a more valuable peer consulting experience for participants. Firstly, we reviewed the issues that emerged from assessing Session 1 outcomes and the reasons for participation and non-participation, including:

- managing and balancing priorities

- site and forum format

- technical difficulties

- relevance of the session topic

- changes in the participant group

Adding to this list, our early discussions of reforming the sessions revealed that the involvement of SMa, SMb, and P18, who held a dual position within $\mathrm{CI}$ that afforded additional responsibility and authority, might also have an inhibiting effect on session participation and engagement with particular consulting topics. SMa noted that their involvement could bring some level of authority relationships into the Session forums, and while this may positively influence a session and "warm [participants] up to a higher level of involvement", it may also influence others more negatively, and "warm them up to exposing less of themselves". Taking this idea further, SMb described their direct involvement in the forum discussions:

\begin{abstract}
"One of the things we could do, if we decided that SMa and P18 and I should be involved in this, is that we could model that process in a way; contributing an idea and then coming back and saying: 'actually, I am not so sure about that idea I said now, what about maybe this... whatever'. So, we are kind of starting to encourage a tone of it being all a bit more about ideas, rather than certain answers." (SMb)
\end{abstract}

We agreed that it was likely that the modelling of such online communication behaviour would have a positive effect on the nature of discussions and that in future $\mathrm{SMb}$ would put this into action if/when appropriate. 
Discussion with SMa and SMb about the possibilities for Session 2 revealed a polarisation of thoughts on moving forward with the project. For example, SMb took the perspective that it was vital to continue with Session 2 to reinforce the experimental nature of the project and ensure that we didn't perpetuate a sense of failure amongst participants:

"...the fact that it continued, as opposed to being just dumped after one. You know, saying here's number 2, the expectation isn't that we get perfect participation first time, the expectation is that this is something that builds and evolves. And so, letting people know, well, this is where you're starting from. We're learning how to find a way to use this that is actually useful to you and you're learning about how to participate in this in a way that's valuable. So, having a bit of a conversation like that with people, I think would be good too. Not for people to feel like there was a great 'failure' or a big disaster about it." (SMb)

In contrast, SMa was of the opinion that it we should pause and spend more time analysing why Session 1 had low participation, and what could be done to improve this:

"I could probably suggest some changes to increase participation, so let's look at that one first. I think the changes to increase participation would be that, it would be a one-to-one telephone call and conversation and a very personal approach, with some support around technical issues for those that are there. I think that at this stage there's almost a risk that that kind of conversation could start to feel coercive, in the sense that a number of people have, sort of given it their best shot."

The potential for this type of approach to be perceived as coercion, combined with the fact that I would not deem a phone call of this nature online peer consulting (as previously defined) meant that, although a future option for CI to address, this approach fell outside the scope of my project.

SMa also gathered feedback (through informal conversation) from several participants on their preference for Session 2 to go ahead and reported "in the three 
meetings that I've had there were two groups and, what they were saying was no." SMa subsequently determined that we should abandon Session 2, although also noted this decision was "not about the project it's more about the structure of [Manager] work flows at the moment." SMa revisited this issue again, stating:

"I think that if the structure of the work was different, [online peer consulting] could still be a good, you know, a more workable thing. But given the time pressure that I'm hearing about, and... it just feels as if there's not the space for them to engage in the kind of reflective conversation that we would be hoping for through this medium." (SMa)

Similarly, many participants commented on the structure of their role and how this added to their preconceptions of peer consulting. P15 illustrates this, and also highlights the important link between the nature of their role and the formalisation of particular professional development processes:

"I think the nature of the [Manager] role means that we don't necessarily value spending time in thinking about and talking about our practice, unless it's sort of formalised. Like supervision or the telephone conferences."

As restructuring the Manager role and/or core CI workflows was outside the scope of my research project, I took this as a key indicator that Session 2 was unlikely to yield significant increases in participation within the current organisational situation.

Therefore, after assessing all the available data and engaging in many discussions we decided to discontinue the series of sessions as initially set out in the Session Structure document (see Appendix J). As such, further formalising of the online peer consulting sessions did not occur and we concluded the reforming activity.

\subsubsection{Reconfiguring systems}

As described in Chapter 4 (sections 4.4.2 and 4.4.3), changes to the online peer consulting session plan necessitate changes to the configuration of the supporting online tools. At CI, the high level of informal peer consulting activity that occurred at 
the Experimenting stage (see section 6.3.1 above) compared to the significantly lower level of engagement in formal peer consulting of Session 1 showed that CI participants preferred consulting informally on issues of practice raised by peers as and when necessary, as discussion with two participants revealed:

"I'd really like to have something like Blackboard where there is ongoing discussion. Where people can pose questions or come up with good ideas, that kind of thing. I am interested in doing that" (P2).

"You know, like before when we were on the discussion board and there was that sort of flow of conversations between [Managers]? (P4)

Therefore, we undertook to reconfigure the Regional Practice Managers website to support ongoing, informal peer consulting. This involved reorganising the various forums on the site so that the Hot Topics forum was most easily accessible to participants, and re-naming this forum to Current Hot Topics to provide some differentiation from the Experimenting stage. Once I communicated to participants that the forum would remain available for them to use as they liked, activity resumed within one day. I had hoped that greater use of this forum by all participants may then initiate a second formal peer consulting session, although this did not eventuate.

\subsubsection{Refining stage summary}

CI entered the Refining stage with some experience of online peer consulting obtained through training at the Structuring stage and informal online discussions of the Experimenting stage. Building on this, some Managers extended their peer consulting practice by participating in Session 1 and sharing their reflections on the session resource through posting one discrete message each to the discussion forum. However, as the majority of Managers did not engage in Session 1 we concluded that peer consulting (as defined in Chapter 2 and described in earlier sections of Chapter 6) did not take place. 
Although CI did not extend their online peer consulting practice at the Refining stage, discussing and assessing Managers' peer consulting preferences and activities was a valuable exercise that revealed many factors inhibiting their participation, and also highlighted their desire to continue informal consulting, as in the Experimenting stage. Subsequently, we reconfigured the discussion forums of their online space to support informal consulting which prompted further activity.

CI did not achieve the intended Refining stage outcomes of formalising their online peer consulting programme (in terms of both the process and supporting site) and undertaking a series of online peer consulting sessions. Determining that the current organisational situation could not support continued formal online peer consulting led CI to the Resolving Point at which they were suspended, for a time, between the Experimenting and Refining stages as they took part in ad hoc unstructured peer consulting of a collaborative nature. Therefore, their progression through the stages of peer consulting transition arrested at the Refining stage.

\subsection{Chapter Summary}

CI initiated their move towards online peer consulting in order to relieve the professional isolation felt by Regional Practice Managers. In particular, they were driven by objectives of improving the connectedness of the Manager group and complimenting national face-to-face meetings with more regular online interactions through which region-specific knowledge could be shared across the organisation.

CI's journey through the stages of Peer Consulting Transition included several notable differences to the generalised model of Chapter 4. Key differences are summarised here, and comparisons are drawn between unique aspects of CI's activities at each stage. 


\subsubsection{Defining stage elements}

At the outset of this research, CI was keen to create and promote their vision for online peer consulting, as a way of introducing and 'warming staff up' to the general concept. Therefore, CI undertook this activity as a precursor to determining the current peer consulting practices of Managers and documenting their ICT support. As an established organisation with entrenched professional development policies and practices developing the concept first was appropriate for CI, and allowed some awareness of the project to filter through the organisation prior to my involvement at their national meeting.

As their understanding of both current peer consulting practices and the desired state for online peer consulting continued to develop, CI suspended other activities and revised their general concept many times. In contrast, Chapter 4 describes Defining stage activities ending with the development of the online peer consulting concept. Indeed, section 5.1 (Chapter 5) discussed how AC developed their online peer consulting concept once current peer consulting practices and ICT support had been determined, which led to the development of a more stable concept that was better able to guide activities at later stages of Peer Consulting Transition. This is summarised in Table 16.

\section{Model of Peer Consulting Transition}

- Developing the online peer consulting concept

:

- Mapping current peer consulting activities

- Documenting current ICT support

\section{CI's Peer Consulting Transition}

- Developing the online peer consulting concept

- Mapping current peer consulting activities

- Documenting current ICT support

Summary: At the Defining stage CI undertook the same activities as presented in the generalised model, in a different order.

Table 16: Comparison of Defining stage activities 


\subsubsection{Structuring stage elements}

As CI has many stable internal processes, we focused on creating a robust strategy for introducing online consulting to Managers. This involved CI moving sequentially through the activities of planning for sessions, and selecting, configuring and training Managers on supporting ICT. This measured approach was deemed more likely to be effective at CI, with the combined result of readying CI for online peer consulting. Hence, the point at which CI successfully completed Structuring stage activities was deemed the Readying Point, and prompted them begin the Experimenting stage.

The general state that CI reached at the conclusion of the Structuring stage differed somewhat from that in the generalised model of Chapter 4 (see table 17). Section 4.1 describes the heightened interest and excitement of an organisation that reaches this point, (as opposed to simple preparedness) similar to the experience of AC (see Chapter 5, section 5.2.3). It is this Rousing Point that propels them to the third transition stage.

\section{Model of Peer Consulting Transition}

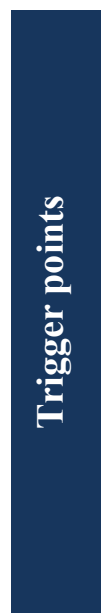

- $\quad$ Planning for sessions and introducing ICT reinforces the potential value of online peer consulting

- $\quad$ Using ICT builds confidence and improves understanding of the concept

- $\quad$ Further interest in and excitement for online peer consulting exists, and the Rousing point is reached.

\section{CI's Peer Consulting Transition}

- $\quad$ I was equipped with a detailed plan and live site that enabled them to action online peer consulting training sessions

- CI Managers gained the information and skills needed to participate in online consulting

- $\quad$ CI reached the level of preparedness of the Readying point, and moved on to the next transition stage.

Summary: The culmination of Structuring stage activities saw CI reach the Readying point, while the generalised model concluded this stage with the Rousing Point.

Table 17: Comparison of Structuring stage Trigger points 


\subsubsection{Experimenting stage elements}

In this stage CI undertook to make changes to both the plan for sessions and the set up of the supporting website. These activities unfolded concurrently, as we switched between altering an aspect of the plan and assessing whether the previous site configuration supported the revised plan or required changes. Here, I grouped CI's actions into the activity: restructuring sessions. Ideally an organisation would make all revisions to the session plan, and then assess the extent to which the chosen online tools supported it. See Table 18 for a summary of these points.

Taking a more holistic view may illuminate ways to reconfigure systems to address multiple session changes, potentially reducing the time and cost involved. In some cases where substantial revision of the session plan is made, complete replacement of ICT may be necessary. Addressing changes to the session plan and ICT separately would eliminate unnecessary re-development of redundant ICT.

\section{Model of Peer Consulting Transition}

- Participating in informal online peer consulting

- Altering the plan for sessions

- $\quad$ Reconfiguring systems

Summary: At the Experimenting stage CI combined the activities of altering their session plan and supporting website, which are separated in the generalised model.

Table 18: Comparison of Experimenting stage activities

\subsubsection{Refining stage elements}

The intended objective of the fourth transition stage is for an organisation to move through a programme of online sessions, incrementally altering session structure and supporting ICT. The resulting session structure and ICT configuration will ultimately 
become formalised to a level necessary for Embedding online peer consulting as an institutional professional development activity (at the final transition stage). For this to occur a learning community must actively engage in collaborative discussion at each consulting session, evolving the nature of their interactions as they proceed. Thus, Chapter 4 (section 4.4) presents the Engaging stage with corresponding activities of participating in and formalising sessions and refining ICT set up.

As described in section 6.4 above, CI's journey of actioning formal consulting did not follow this path (see Table 19). There was a low level of participation in session 1, no subsequent sessions held and a shift back to informal, ad hoc consulting of the Experimenting stage. Consequently, CI undertook a period of very little engagement in online peer consulting, and instead worked to refine their vision for and general approach to introducing online peer consulting to Managers. Thus, I have labelled this the Refining stage, with the key activity being reforming (as compared to formalising) online peer consulting.

A key decision point of the Refining stage was determining that discontinuing formal sessions was the most appropriate action. The activities of reforming sessions and reconfiguring ICT facilitated CI's shift back to informal, ad hoc consulting. This general process forms the Resolving Point, which concluded the Refining stage activities at CI. In contrast, after working through the Engaging stage activities as outlined in the generalised model an organisation would have a workable concept and created the environment in which both formal and informal online peer consulting could successfully continue, which I termed the Crystallising Point. Table 19 illustrates these differences. 


\section{Model of Peer Consulting Transition}

The core focus of organisational activity is participants' engagement in a series of formal online peer consulting sessions, hence the title of the Engaging stage.

- Participating in peer consulting sessions

- Formalising peer consulting sessions

- Refining systems set up

- Completion of activities results in an accepted and operationalised peer consulting concept coming together with an organisational and online environment conducive to continued online consulting. This

Crystallising point guides an organisation to begin the process of embedding online peer consulting across the organisation.

\section{CI's Peer Consulting Transition}

Stage 4 is titled the Refining stage, as CI worked to reassess their vision and plan for online peer consulting, and honed their overall approach to supporting Managers' engagement.

- Performing formal online peer consulting

- Reforming online peer consulting

- Reconfiguring systems

- Based on participation level CI decided to discontinue formal sessions and undertook actions to move Managers back to informal, ad hoc consulting. This general process forms the Resolving point, which concluded the Refining stage activities at CI.

Summary: At the Refining stage CI focused on honing their approach to online consulting, as distinct from the focus on continued and extended engagement (Chapter 4). CI then reached the Resolving point and discontinued formal online peer consulting sessions.

Table 19: Comparison of Refining stage components

In summarising the above discussion, Figure 14 (below) illustrates the prominent stages, activities and trigger points (both similar to and distinct from the generalised model of Chapter 4) that CI achieved in their journey towards Peer Consulting

\section{Transition.}




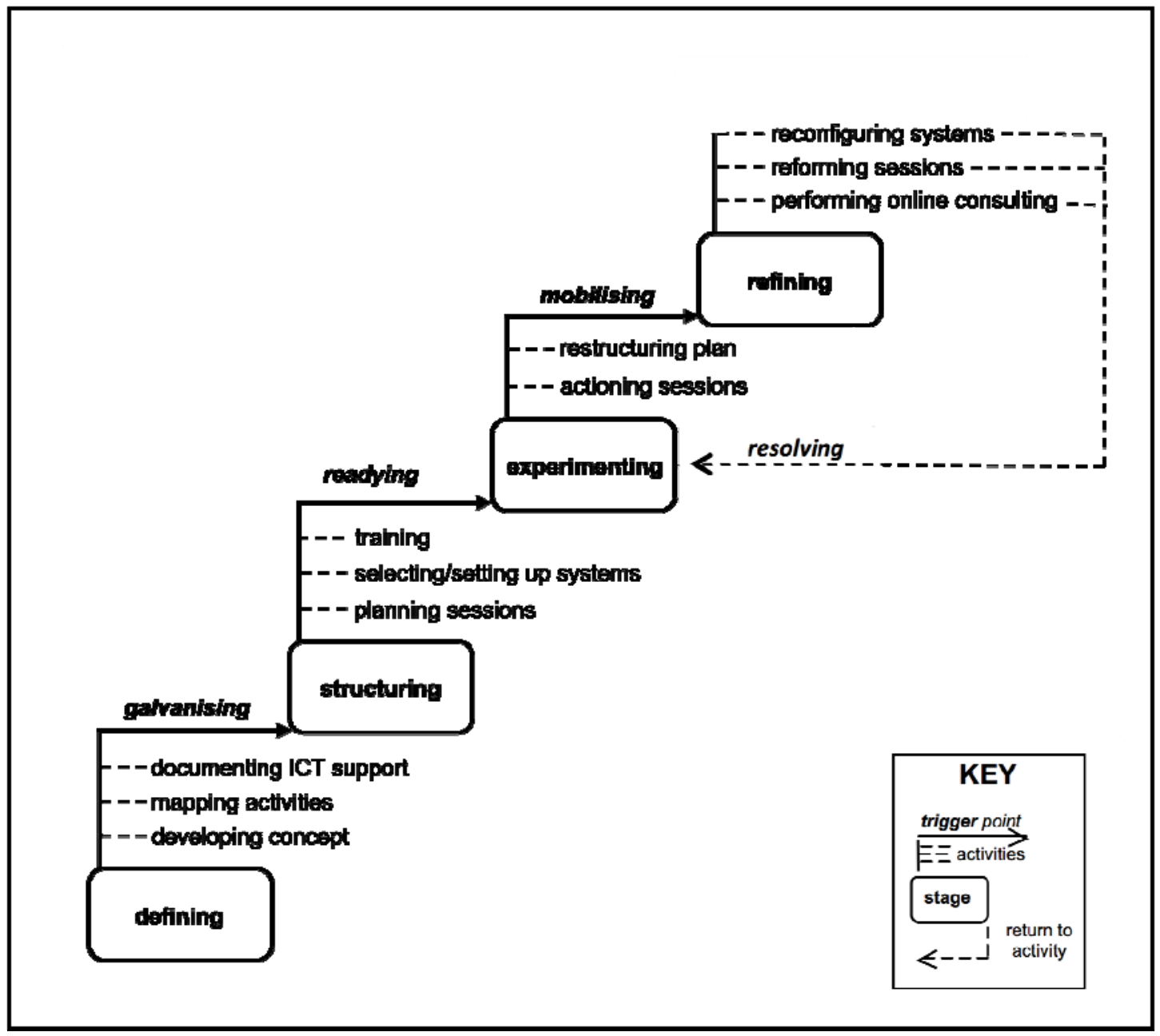

Figure 14: The Stages of Peer Consulting Transition at CI

Several issues occurred within and across all transition stages and impacted CI's ability to introduce and/or engage in online peer consulting, including:

- managing and balancing Manager priorities

- site and forum format

- technical difficulties

- relevance of session topics

- changes in the participant group

- involvement of Senior Managers

- the structure of the Regional Practice Manager role

- general CI workflows

These issues arose at various times and impacted participant activities in different ways throughout the stages of CI's transition towards online peer consulting. Of most importance was the unique combination of issues at the Refining stage that hindered 
session participation and ultimately arrested CI's progression towards instituting online peer consulting.

As a result, CI did not reach the final stage of transition and online peer consulting did not become an enduring professional development process. Instead, CI re-visited the Experimenting stage and continued ad hoc, unstructured (yet still collaborative) online peer consulting. Although their progression through the stages of Peer Consulting Transition was cut short, this chapter has shown that CI gained valuable experience and knowledge that they can leverage in the future. It is also important to note that their move away from the Refining stage and failure to progress to the activities of embedding online peer consulting within the organisation does not suggest that MST was unsuccessful in guiding media selection. Rather, it was elements of CI's organisational structure and culture that had a more prominent impact on their decision to suspend peer consulting transition. 


\section{Enacted Social and Technology Structures: ICT Use and Organisational Change}

\subsection{Chapter Overview}

The analyses in Chapters $5 \& 6$ centred on each organisation's pathway through peer consulting transition, and how they had moved through stage activities and reached each Trigger Point of the Model of Peer Consulting Transition. Within the Engaging stage, participants from AC and CI interacted through online peer consulting sessions to advance their professional learning. Throughout the sessions, various elements of organisational culture and structure influenced participant actions, particularly their use of ICTs, which subsequently also influenced the nature of their peer consulting exchanges. As further understanding of such elements would contribute to answering the primary research question of: how do NZ social services organisations apply online ICTs for the professional development of staff? ${ }^{19}$ I was prompted to focus intensely on revealing and exploring in detail the prominent organisational factors at $\mathrm{AC}$ and $\mathrm{CI}$ that had an ongoing effect on participant behaviour, online peer consulting sessions and their progress through the model.

Kehrwald (2008) described online learning environments as "social-relational systems driven by the exchanges between individual actors in the environment" (p. 90). My desire to analyse organisational factors 'in action' within online peer consulting sessions led me to look more closely at such exchanges, and to discover structuration theory and then adapted structuration theory (AST), and through these earlier frameworks to realise the potential value of applying Orlikowski's Technology-inPractice (TiP) framework. Within this chapter, I describe how the TiP framework provides a valuable second lens through which I identify and examine prominent organisational factors, and assess their impact on online peer consulting transition and organisational change at $\mathrm{AC}$ and $\mathrm{CI}$. The chapter begins with an outline of the

\footnotetext{
${ }^{19}$ The research questions guiding this study are given in Chapter 1.
} 
development of the TiP framework, and a discussion of its key elements. The relationship between the Model of Peer Consulting Transition and the TiP framework is next described. The most significant part of this chapter is section 7.3, the detailed description of the predominant TiPs evident within AC and CI, and how recurrent structures of technology use either led to further transition activities, or abandonment. The chapter concludes with reflection on the application of the TiP framework, and a summary of the identified organisational factors at work within the research settings.

\subsection{Technology-in-Practice Framework}

Historically, there have been many approaches to researching the relationship of technology to organisational structure, approaches and outcomes. Orlikowski (2000; p. 404) summarises some key research perspectives as: contingency theory, strategic choice models, Marxist studies, symbolic interactionalist approaches, transaction-cost economies, network analyses, practice theories, and structurational models. More recently, increased attention has been placed on the development of concepts and models of innovation, learning and improvisation to research the role and impact of technology on organisations (Orlikowski \& Barley, 2001). As the TiP framework applied in this study stems from earlier work on structuration, particular attention is placed on these perspectives, as described in the remainder of this section.

In understanding technology, structurational models have been strongly influenced by the sociological theory of social constructivism (Massa \& Testa, 2005). Recently, the importance of social context in the study of technology and organisation has been highlighted, prompting a rise in social constructivist approaches that acknowledge the social nature of technology. Such approaches share the view that "technology can only be understood in the context of individuals and groups comprehending, interpreting, using and engaging with those technologies" (Dery, Hall \& Wailes, 2006; p. 231). 
Orlikowski and Barley (1991), among others (such as Tolbert, Rose and Barley) have sought to strengthen social constructivist approaches to technology, making three important contributions. Firstly, a focus has been directed on elements of technology, or technological artefacts that influence agency. Secondly, they have extended the assertions that technologies become 'stabilised' over time, and presented the argument that processes of social construction are ongoing and never truly 'fixed'. Thirdly, they situated technology firmly in practice, bringing work and work practices back into the analysis of technology within organisations (Dery et al., 2006). An outcome of this work is the Technology-in-Practice framework (Orlikowski, 2000) as a 'practice lens' for studying the ongoing use of technology in organisation. An overview of the development and key elements of this framework is given here.

The key premise of the TiP framework draws from elements of structuration theory. Structuration theory was developed by the seminal social philosopher Anthony Giddens $(1979,1984)$ and examines the process of putting social 'structures' into action. Structuration is the process by which social structures (whatever their source) are put into action (DeSanctis \& Poole, 1994). Central to structuration theory is the mutual dependence of agency and structures. Specific to this study, agency refers to participant's interaction with the system as they participated in online peer consulting, and structure is the combination of various rules and resources that were brought about by systems use and acted to structure further systems use.

Within TiP, structures exist only as agents apply them, and they are both the medium and outcome of action. In this way, the social order of a system is maintained over time (stability) and yet has the capacity to adapt (change) as actors modify structures in the course of their interactions with one another. At a basic level, structuration theory proposes that a complete understanding of social interaction require incorporating explanations of both the structure of relationships and the dynamics or processes of relationships. The processes both take place in social structures, and shape those structures over time (DeSanctis \& Poole, 1994). 
Structurational approaches appealed (and continue to appeal) to organisational and IS/IT researchers due to the emphasis placed on the interaction of technology and the social processes of technology use, which highlight that multiple organisational outcomes can result from the introduction of the same technology (DeSanctis \& Poole, 1994).

During the period 1982 - 1994 DeSanctis \& Poole worked to extend structuration theory to address the mutual influence of technology and social processes on groups. They argued that previous structurational approaches were limiting as they gave only weak (if any) consideration to information technology and systems, were exclusively focused at the institutional level, and relied on purely interpretive methods failing to address the causal relationships that some IS researchers wished to explore.

DeSanctis and Poole (1994) developed Adaptive Structuration Theory (AST), described as a high-level theory for studying the role of technology in organisational change. AST was evolved from structuration theory, although is based on a number of propositions that depart from Giddens' initial intent:

- Social structures serve as templates for planning and accomplishing tasks,

- Designers incorporate some of these structures into the technology (with the result that the structures may be reproduced or modified),

- Thus creating new structures within the technology

AST was originally developed for studying the role of IS/IT, particularly group decision support systems (GDSS) in organisational change (especially within small groups). Pozzebon, Diniz \& Jayo (2009) described AST as an influential stream of IS research, carried out by prominent researchers, including Chin et al. (1997), Fulk (1993), Majchrzak et al. (2000), Maznevski and Chudoba (2000), and Miranda and Bostrom (1993-1994, 1999). Such researchers have used AST to assess the organisational change process from two key vantage points:

- The types of structures that are provided by IT/IS

- The structures that actually emerge in human action as people interact with these technologies 
With AST both the structure of the technology and the emergent structure of social action could be studied. A broad 'metapattern' of social structure then emerged within a group, influenced by use of the new technology. Concisely put, AST is a model that "describes the interplay between advanced information technologies, social structures and human interaction" (DeSanctis \& Poole, 1994; p. 125). While human action (agency) remained central to AST approaches, it was largely through embedding structures within a technology during development, or 'appropriating' those structures through technology use (Orlikowski, 1992). Giddens held the position that structure is virtual, existing only in its instantiation (when it is translated into action). In contrast, AST views structure as being embedded within technology, which appears somewhat inconsistent with Giddens's original intent.

Orlikowski provides another structurational perspective on technology, developing an analytical framework that is grounded in structuration theory. Her work within large, corporate organisations led to the development of a practice perspective that incorporates the duality of technology, or that agency is instantiated through interaction between knowledgeable people and technology (Orlikowski, 1995). Orlikowski commented on the wide range of different uses and adaptations of technology that are possible when human agents engage with elements of a technological artefact, concluding that the intended use of 'designed' technology and actual emergent 'technology-in-use' were not always a direct fit (Orlikowski, 1995). The model of technology-in-use was a significant development of this research, described as the situated patterns of use involved in utilising a technological artefact (Orlikowski, 1995; p. 5).

Orlikowski (2000) further extended the structurational perspective and developed the Technology-in-Practice framework. It is important to note here that there exist two important differences between this framework and AST. First is the distinction between embodied versus emergent structures. Orlikowski argues that structures are not built into technology, but they are instead enacted as people interact with technology in their (work and social) practices and the enacted structures shape their use of that technology in an ongoing process. This is a departure from the view of 
AST that technologies embody structures as the practice view sees structures as emergent, through a person's repeated interaction with that technology. As noted by many researchers, the outcome of any IS/IT tool is emergent, since there will always exist opportunity for improvisation in ongoing technology use. ICT tools are expected to be used in different ways, either ignoring certain properties, working around them, or inventing new ones that may go beyond or even contradict designers' original expectations and intents (Prakash \& De, 2007; Massa \& Testa, 2005). Drawing on Giddens' (1984) concept of structuration, Orlikowski is explicit that these structures (ie, technologies-in-practice) are never completely fixed or stabilised; while they might be reinforced through the repeated use of technology in particular sorts of ways, they are always open to change (Dery et al., 2006)

The inclusion of enacted rather than appropriated structures is a second key difference. Orlikowski argues that, rather than starting with technology and examining how actors appropriate it's embodied structures, the practice lens instead begins with human action (agency) and examines how emergent structures are enacted through recurrent interaction with a particular technology. People always can (even if they do not) redefine the meaning, properties and application of a given technology after development or implementation (Prakash \& De, 2007; Pozzebon et al., 2009). Over time, particular properties of a technology become implicated in an ongoing process of structuration, and therefore a particular structure of technology use emerges (Orlikowski, 2000).

Thus, rather than focusing on technological aspects, embodied structures and their influence on use, this view starts with human behaviour and the enactment of emergent structures in the use of technology (Massa \& Testa, 2005). Technology structures then move away from being external or independent of people's actions, embodied in technologies and waiting to be appropriated. They are virtual, congruent with Giddens' writings, emerging from people's repeated interactions with particular technologies as situated in their practice (DeSanctis \& Poole, 1994). In her writings, Orlikowski stressed that the relationship of human agency and technological artefact is essential to understanding actual technology use. 
As organisational members use technology to engage in their work practices, sets of rules and resources are (re)constituted in their recurrent interaction with that technology (Orlikowski, 2000). While the importance of the object or technical artefact is acknowledged through the assessment of resources (facilities of a technology), Dery et al. (2006) argue that it is only through their enactment in practice that their impact on organisations can be understood. This highlights the need to focus on technology in practice to examine how technology structures users' actions, and influences people and organisations (Orlikwoski, 1995 \& 2000; Dery et al., 2006). The analyses and discussion of section 7.3 of this chapter describe such enactment and organisational impacts, as observed in the practice of participants at CI and AC.

Figure 15 is a graphical representation of the Technology-in-Practice framework, and Table 20 elaborates key elements. 


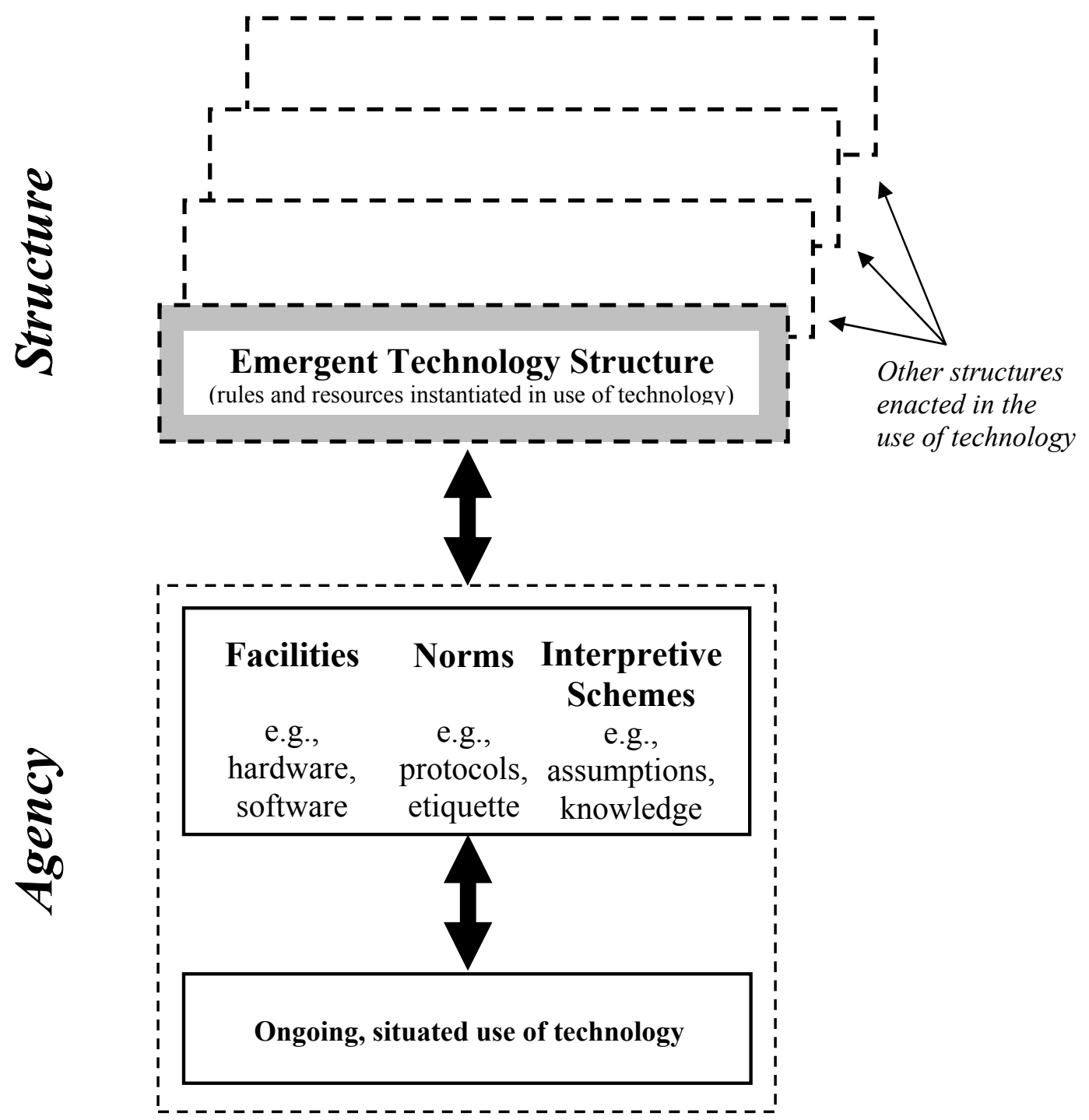

Figure 15: Enactment of Technologies-in-Practice (adapted from Orlikowski, 2000) 


\begin{tabular}{|c|c|c|}
\hline & Element & Description \\
\hline & Interpretive Schemes & $\begin{array}{l}\text { Rules that the reflect knowledge of the work being done, } \\
\text { including skills, power, and expectations }\end{array}$ \\
\hline$\frac{1}{2}$ & Norms & $\begin{array}{l}\text { Rules that define the organisationally sanctioned way of } \\
\text { executing that work, including values, standards, and policies }\end{array}$ \\
\hline & Facilities & $\begin{array}{l}\text { Resources to accomplish that work, such as the properties } \\
\text { (features/functions) comprising the technological artefact }\end{array}$ \\
\hline & Interaction & The actions of knowledgeable people within an organisation \\
\hline$\hat{3}$ & $\begin{array}{l}\text { Emergent technology } \\
\text { structure(s) }\end{array}$ & $\begin{array}{l}\text { The specific set of rules and resources that are enacted } \\
\text { through practice (technology use) }\end{array}$ \\
\hline$\frac{1}{2}$ & $\begin{array}{l}\text { Other organisational } \\
\text { structures }\end{array}$ & $\begin{array}{l}\text { Other enacted social structures, including authority structures, } \\
\text { organisational culture, incentive schemes }\end{array}$ \\
\hline
\end{tabular}

Table 20: Elements of the Technology-in-Practice Framework (adapted from Orlikowski, 2000)

Figure 15 displays a two-way arrow between the core elements of agency, and most importantly between elements of agency and structure. This two-way movement represents the way that structures of technology are constituted recursively; they come about when people repeatedly interact with particular features of a technology, the emerging structure of technology brings about a combination of rules and resources, which then shapes their future interactions (Orlikowski, 2000).

In summary, the use of technology by organisational members becomes structured by the existing facilities, norms and interpretive schemes (rules and resources) available. Such structuring enacts a specific set of rules and resources in practices that then serve to structure future technology use. As a result, over time people constitute and reconstitute a structure of technology use, and thereby enact a distinctive technologyin-practice.

Orlikowski called for researchers to apply the TiP framework in various research contexts to focus on how people use technology in their day-to-day activities, not on 
the technology itself. She believed that this could yield important insights into the influence of technology use on organisational change and effectiveness. Indeed, Boudreau \& Robey (2005) cited Orlikowski's (2000) conclusion that transformations of organisational practice were enacted in use rather than caused by technology. They went further to argue "as users enact technologies in response to their local experiences and needs, significant organisational changes may result over time" (Boudreau \& Robey, 2005; p. 4).

As a particular structure of technology is enacted and changes in practice begin to occur, other organisational structures are also enacted (see Figure 15). For the purposes of this study, these organisational structures are deemed to be the organisational factors that impacted on participant's progression through the model of peer consulting transition. It is important to highlight such organisational factors, as the organisational environment is one key determinant of the success of the introduction, adoption and continued use of new innovations, as described within this chapter.

In her recent writings, Orlikowski suggested that the TiP framework should be adopted for other types of organisations, particularly moving beyond the large corporate and multinationals to smaller, national or local organisations where technologies are a more recent 'add on' to organisational processes (Orlikowski, 2000). However, since its inception few researchers in the wider IS/IT discipline have applied this framework to assess the impact of human agency on organisational outcomes. Section 7.2 describes how the TiP framework has been applied in this study.

\subsection{Application of the Technology-in-Practice Framework}

As described above, the TiP framework provides a lens through which the ongoing situated use of technology and the structures that both emerge from and continue to shape technology use can be studied. Within this research, the TiP framework has 
been applied within each stage of peer consulting transition at Associated Counselling (AC) and Counselling Incorporated (CI) to elaborate the organisational or factors present that encouraged or inhibited programme transition.

Chapter 4 outlined the Model of Peer Consulting Transition, and Chapters 5 and 6 described the specific AC and CI cases respectively. Through these chapters, certain key insights were drawn out, analysed and described. A deeper level of analysis is possible through the application of the TiP framework, particularly of the interaction of participants with features of technology, and the social structures that emerged from this interaction and influenced ongoing technology use.

Dery et al. (2006) stated that Orlikowski's TiP model pays little attention to the important relationship between technologies-in-practice and other structures, and argued for further adaptation to promote the importance of other organisational social structures. Somewhat earlier, DeSanctis and Poole (1994) argued for the importance of considering other sources of structure that exist within the organisational environment within which technology is used, specifically listing "elements of the wider organisational environment".

Addressing this call and in light of Boudreau and Robey's confirmation that aspects of organisational change come about through technology use, I have applied the TiP framework to elucidate the organisational structures that were enacted as members of $\mathrm{AC}$ and $\mathrm{CI}$ used particular information systems for online peer consulting. Specifically, this application of the TiP framework has shed light on the organisational factors that supported $\mathrm{AC}$ in moving through the five stages of the Model of Peer Consulting Transition, and helps to explain why CI suspended progress through the model at the fourth stage, Engaging.

Through analysing the use of technology within a particular time period at each organisation, I was able to observe a particular set of rules and resources emerge as predominant, ie a predominant TiP was constituted. As participants of $\mathrm{AC}$ and $\mathrm{CI}$ 
enacted this TiP, they drew on and were constrained or enabled by other social/organisational structures existing within the organisations. Thus, as other organisational structures that were enacted I was able to conduct a more detailed description of the organisational factors (as elements of the organisational structures) that were both brought about by and influenced technology use.

An understanding of the nature and impact of these organisational factors acts as a powerful assist to organisations considering the introduction of new innovations. At $\mathrm{AC}$ and $\mathrm{CI}$ the organisational factors that emerged were closely linked to the organisational norms and assumptions present, and were enacted through early technology use and then acted to constrain further technology use.

To demonstrate the application of the TiP framework, section 7.3 provides a description of the predominant technologies-in-practice that emerged within the Engaging stage of peer consulting transition at each participant organisation. The set of technologies-in-practice build a picture of the organisational factors that supported $\mathrm{AC}$ in progressing through the developmental stages and extending their peer consulting programme, and organisational factors that caused CI to falter, then discontinue programme development.

\subsection{Technologies-in-Practice at the Engaging Stage}

At each organisation, the Engaging stage involved several key activities that combined to form the period of engaging in online peer consulting sessions, and reviewing and refining both the peer consulting session plan and systems use. This section focuses specifically on the nature of the interaction that members of each organisation had with various technologies to support group peer consulting at this fourth stage of peer consulting transition. 
The lens of technologies-in-practice (TiP) is used to describe the nature of this technology use. The TiP framework consists of two types of elements: rules and resources. These are specifically defined within the Engaging stage as knowledge and experience of professional development and the organisationally sanctioned ways that professional development was delivered at each organisation, and the technologyrelated resources that were used to support and deliver peer consulting (as a professional development activity) within each organisation. Within the Engaging stage, various rules and resources emerged that coupled with participants evolving experience with and understanding of technology supported professional development.

The factors that influenced participants' use of the various systems within the online peer consulting sessions are placed within the two categories of agency (participant's interactions with the systems) and structure (emergent sets of rules and resources).

Through the following descriptions, each of the key factors that influenced systems use within the online peer consulting sessions is described, and an overall picture of the most prominent TiP that emerged at $\mathrm{AC}$ and $\mathrm{CI}$ is provided (sections 7.3.1 and 7.3.2 respectively). The use of the TiP framework enabled me to explain the organisational factors in play that show why one organisation was more successful than the other in transitioning peer consulting to the online environment.

\subsubsection{Technology-in-Practice at AC}

At the Engaging stage, participants at AC took part in a series of six online peer consulting sessions. In addition to the ad hoc peer consulting of the Familiarising stage, systems use at this stage was extended and refined as the series of sessions progressed. This section describes the nature of the ongoing interaction of members of AC with the online collaboration system. As they used various functions of the system to support their peer consulting sessions, their actions and interactions were shaped in particular ways. The technology structure that emerged shaped further technology use, 
enabling and at times constraining their peer consultation. Thus, a reciprocal relationship between agency and structure was observed.

The lens of technologies-in-practice is used to explain this interaction and the interplay between elements of agency and structure and highlight the key organisational factors that influenced technology use. I have labelled the key outcome of this stage the Collaborative TiP. Within this, a number of key organisational structures were enacted that are discussed here. Firstly, the Cooperative Culture of $\boldsymbol{A C}$ is described in detail. A description of the emergent elements of participants' actions (agency) and the technology structure (structure) are provided, and an overview of these elements is given in Table 21 . The elements of the factors of the Distributed Nature of AC and the Adaptable Communication Structures are given within Table 22 and 23 respectively. Following these descriptions, all elements of all three factors observed within AC are presented in Figure 16.

\section{The Cooperative Culture of $A C$}

As described earlier (see Chapter 5) the management team of AC aim to engender a culture of cooperation amongst the staff. This desire for the cooperation needed for open communication, knowledge sharing and professional development was found to be an important influencing factor on the nature of technology use by members of AC as they moved through the series of online peer consulting sessions.

Most members of AC did not have a high level of knowledge of or experience with IS/IT at the outset of this project, which contributed to their apprehension towards technology use. Despite such apprehension, the majority of the participant group showed willingness to trial the online system and pioneered its use within AC. For example, the first online peer consulting session could have been held as a more traditional face-to-face regional supervision meeting. Instead, participants drew on the cooperative culture of AC and showed their enthusiasm towards experiencing the online system, choosing to hold this session online and include peers from other regions, as explained by $\mathrm{P} 4$ : 
"We have a larger group, and if we added in some one else from Chatterbox we though it might get a little unwieldy. But then we decided to try it anyway, and see what it was like"

Through this initial systems use, participants actually drew on their lack of IS/IT knowledge and experience as an encouraging factor, and questioned how the particular features of the system operated and the application of certain features in supporting group consulting and cooperation among staff. One member of AC describes this as the motivation for participating in their first online peer consulting session, stating: "I guess the most immediate thing was I wanted to come away with an understanding of what Chatterbox actually did. And how it could be useful for our staff' (P4).

While at the outset such questioning revealed their lack of knowledge of online systems, their forward thinking reinforced their openness towards technology use and advised further systems use during later sessions. As their initial interaction with the online system was a positive experience, most people found their openness towards technology use reinforced, simply put by P4: "I think it was more in the measure of 'oh, this does work, and looks like a good idea'”.

As groups of participants used the online system several times, their experience with and understanding of the basic functions of the online systems developed. This developing experience and understanding among the participant group is one key rule that was enacted through ongoing systems use across the six sessions. P2 describes this, after participating in Session 2:

"I still feel like exploring how it's working. I found it very useful and so I am really keen to continue to use it, as are, I think the [other regional] crew" (P2).

Those participants most apprehensive about interacting with the online system, some of whom also indicated some scepticism towards the value that such a system could offer, relied on the support and encouragement of those peers more experienced and 
knowledgeable. In particular, two key participants drew on the cooperative culture of the organisation and were instrumental in demonstrating additional features of the system to others, and leading 'training-in-action' when required. For example, within Session 3 P9 and P2 demonstrated the use of the text function by posting the session agenda. Other participants who were eager to learn how to do this and questioned P9 who then repeated the use of the function and explained how it worked to others. Essentially, this is an extension of the basic training-in-action that occurred at the Familiarising stage (see Chapter 5, section 5.3). P9 described offering such support on two separate occasions, at Session 2:

"We experimented a little bit with the text function after the consultation session. Just to familiarise people with it. For example, Malcolm wasn't sure how to enter text and so we just went through that. He just entered in some text, pretty random sort of information. It wasn't actually used as part of the consultation process" (P9).

and P9 offered support again for a different consulting group at Session 3:

"This is the first session for some of the people doing Chatterbox. So we spent the first part of the session talking about how we might do it. That's everybody toing and fro-ing. But eventually... to be honest in fact I presented the case so that... just to kick it off really, to kick off the session so that there was not so much a model, but so that they could see it being done" (P9).

This pattern of peer support and cooperation was evident throughout the series of online peer consulting sessions, and was highlighted as a key rule instantiated in their recurrent Collaborative TiP. The openness towards technology use of this participant led to them supporting others. In turn, their positive experience of the online system and the Collaborative-Use Practice that was enacted reinforced the organisational norm of peer support. This encouraged participants' acceptance of the new technology and its further use within later sessions and strengthened the cooperative culture of AC. However, this openness did not lead directly to uninhibited acceptance of the online collaboration system, but allowed participants to build experience with the way that it structured their discussions and added value to their practice. Some participants, especially P11 described their early use of the system as bizarre: "it's really bizarre 
when you are in a room with three people and you have to push the button and speak into a mic[rophone] to talk to them".

Through overcoming initial apprehension and being uncomfortable in the online environment, participants developed an individual and collective experience of the online system and the way that it can be used to support peer consulting in this way, participants demonstrated their openness towards technology use. This initial use of the voice and text functions of the system led to particular rules and resources being enacted (agency) and the resulting pattern of use as described above. This technology use, while at first uncomfortable for participants, led to continued use and emergence of the Collaborative TiP. In addition, their use of the online system through the series of six sessions led to a broadening of individual and collective understanding of the potential application of some of the features that they had used as they participated in online group consulting.

In turn, the collaborative technology structure that was enacted through the series of sessions influenced further use of the online system by those who had already experienced it and was an encouraging force for those joining the online peer consulting sessions for the first time. Through ongoing use of the online collaboration system at the Engaging stage, most members of AC continued to reinforce the organisational value of cooperation and their openness to technology use, building specific experience with and knowledge of the basic features of the system.

As they engaged with the system and enacted the Collaborative TiP, an additional social structure of the cooperative culture of $\mathrm{AC}$ was also reinforced. As discussed in Chapter 5, AC hold values around peer support and building a sense of community among staff. Although the organisation is still relatively young and within the start up phase, the cooperative culture is apparent and is emphasised by the organisation founder:

"When we look at the values around [AC], one of the values is that we try and support each other. And so something like this represents another tool for supporting each other" (P4). 
At the Engaging stage, members of AC cooperated within regions and across regions to begin to transition from regional face-to-face supervision meetings to online peer consulting sessions. Within sessions the cooperative culture was also apparent, as participants assisted each other with practice-based issues and also in learning to use the online system. The specific set of rules and resources enacted that highlighted the openness towards technology use of members of $\mathrm{AC}$ and the cooperative organisational culture are depicted in Table 21 below.

\begin{tabular}{|c|c|c|}
\hline \multirow{5}{*}{$\frac{\sum^{2}}{20}$} & Element & Description \\
\hline & $\begin{array}{l}\text { Interpretive } \\
\text { Schemes }\end{array}$ & $\begin{array}{l}\text { - Cooperation supported through peer consulting sessions } \\
\text { - Evolving understanding of the nature of online collaboration } \\
\text { - Openness towards new experiences of using online systems } \\
\text { (and features/functions) } \\
\text { - Limited prior experience using online information systems }\end{array}$ \\
\hline & Norms & $\begin{array}{l}\text { - Cooperative culture of AC } \\
\text { - Value of communication and collaboration } \\
\text { - Supporting colleagues in their professional development } \\
\text { - Organisation wide knowledge sharing performed }\end{array}$ \\
\hline & Facilities & $\begin{array}{l}\text { - Basic features of the online collaboration system } \\
\text { - Basic PC hardware } \\
\text { - Internet connection (dial up and broadband) }\end{array}$ \\
\hline & Interaction & $\begin{array}{l}\text { Participants use features of the online system, as needed to support } \\
\text { group peer consulting }\end{array}$ \\
\hline$\stackrel{0}{\mathbf{s}}$ & $\begin{array}{l}\text { Emergent } \\
\text { technology } \\
\text { structure(s) }\end{array}$ & $\begin{array}{l}\text { Use of the online system for group consulting supports the } \\
\text { cooperative and supportive culture of } \mathrm{AC} \text {, and the Collaborative } \\
\text { TiP is enacted }\end{array}$ \\
\hline$\frac{3}{2}$ & $\begin{array}{l}\text { Other } \\
\text { organisational } \\
\text { structures }\end{array}$ & $\begin{array}{l}\text { - Cooperative organisational culture } \\
\text { - Organisation-wide knowledge sharing }\end{array}$ \\
\hline
\end{tabular}

Table 21: Elements of the Cooperative Culture of AC 
In summary, through the collaborative use of the online system across six online peer consulting sessions, the cooperative culture of the organisation combined with participants openness towards technology use enabled them to add to their understanding of the value of IS/IT to support peer consulting and to develop individual and collective experience with using the online system. The collaborative technology structure that was enacted through systems use reinforced this openness towards technology use, the organisational value of peer support and the cooperative culture of AC.

Within the remainder of this section I describe the remaining factors of the Flexible Nature of AC and the Adaptable Communication Structures in less detail than I have discussed the Cooperative Culture of AC. This is because the Cooperative Culture was found to be the most influential factor and the Flexible Nature and Adaptable Communication Structures are less prominent, although had an impact on AC's progress through the stages of the Model of Peer Consulting Transition. The key elements of the two factors are outlined below, and an overview of the set of rules and resources for each is given within Tables 22 and 23 respectively.

\section{Flexible Nature of $A C$}

Chapter 5 described AC as a geographically distributed organisation, with small groups of staff located within several regions of NZ. Within this organisational structure, the members of AC are unable to meet face-to-face regularly enough and so they have explore more flexible ways of interacting. Here, flexible means more ad hoc, dynamic ways of engaging to meet particular needs at a particular time, as opposed to instituted communication and collaboration processes that are followed. As AC is still within the start up phase and a relatively young organisation, organisational policies, process and agreed practices for organisation wide communication and collaboration (including professional development) are still being developed, which allows staff to adopt flexible and innovative approaches to situations. As AC matures, it is likely that flexibility will give way to increased structure and preferred processes will be followed. 
The flexible nature of $\mathrm{AC}$ was found to have an important impact on the nature of participants' use of technology during the online peer consulting sessions. As previously described, AC offers a specialist service within several regions, resulting in staff spread geographically and the need for the organisation to adopt an effective distributed structure. The distance between staff was often a key inhibiting factor of conventional peer consulting and indeed could also have inhibited online peer consulting also. Instead, as issues of technical and process-related nature emerged, participant groups openly discussed such events and altered their consulting practice as necessary. Thus, the flexible nature of the organisation often ensured that participants continued through the series of online peer consulting sessions. In addition, participants were also flexible within sessions, for example with nominating a case presenter and accepting that peers may need to join a session late or leave early.

As the series of sessions unfolded, it was evident that elements of the flexible nature of the organisation carried over to the nature of the online peer consulting sessions, and participants remained open to change, reviewing each session as it occurred.

Participants' use of technology reinforced the distributed nature of the organisation in a number of ways. For example, groups of co-located peers kept to their usual meeting times and prioritised the inclusion of more isolated peers. This effectively also widened the pool of expertise from which they could gain perspectives, and decreased the professional isolation of members of AC. The key elements of this factor that were found to influence the Collaborative TiP are presented within Table 22 below.

The particular understandings and norms of the participant group combined with particular features of the technology brought about a set of rules and resources that, when enacted through ongoing technology use enabled the Collaborative TiP to emerge. As this structure of technology use was reinforced, other organisational structures emerged that supported the flexible and adaptable nature of AC and encouraged participants to continue through the series of online peer consulting sessions. 


\begin{tabular}{|c|c|c|}
\hline \multirow{5}{*}{ डे } & Element & Description \\
\hline & $\begin{array}{l}\text { Interpretive } \\
\text { Schemes }\end{array}$ & $\begin{array}{l}\text { - Flexible nature of peer consulting sessions } \\
\text { - Expectation that online peer consulting will widen the pool of } \\
\text { expertise available } \\
\text { - Developing understanding of the value of IS in supporting peer } \\
\text { consulting } \\
\text { - Developing experience of using online systems }\end{array}$ \\
\hline & Norms & $\begin{array}{l}\text { - Flexible and adaptable nature of AC } \\
\text { - Value of openness of communication } \\
\text { - Prioritise inclusion of isolated colleagues } \\
\text { - Regional peer consulting performed }\end{array}$ \\
\hline & Facilities & $\begin{array}{l}\text { - Features of the online collaboration system } \\
\text { - Basic PC hardware } \\
\text { - Internet connection (dial up and broadband) }\end{array}$ \\
\hline & Interaction & $\begin{array}{l}\text { Participants use features of the online system, as needed to support } \\
\text { group peer consulting }\end{array}$ \\
\hline$\Xi$ & $\begin{array}{l}\text { Emergent } \\
\text { technology } \\
\text { structure(s) }\end{array}$ & $\begin{array}{l}\text { Use of the online system for group consulting supports the flexible } \\
\text { and adaptable nature of AC, and the Collaborative TiP is enacted }\end{array}$ \\
\hline$\frac{2}{2}$ & $\begin{array}{l}\text { Other } \\
\text { organisational } \\
\text { structures }\end{array}$ & $\begin{array}{l}\text { - Distributed organisational structure } \\
\text { - Regional team meetings }\end{array}$ \\
\hline
\end{tabular}

Table 22: Elements of the Flexible Nature of AC

\section{Adaptable Communication Structures}

As $\mathrm{AC}$ is a relatively young organisation, there is not a long history of how peers interact with each other, and a formal peer consulting process was not followed. However, participants described many aspects of face-to-face interactions with peers that were translated into online peer consulting sessions (see Chapter 5, section 5.2 for a detailed discussion). Specifically, the general format of sessions was similar, as were the desired outcomes of sessions including improved understandings and altered (more effective or safer) practice. 
When comparing their experience of using the online system and engaging in online peer consulting sessions with face-to-face consulting, participants described both encouraging and inhibiting elements. For example, many participants commented on how the online peer consulting system structured their discussions. Initially, the cueing function of the online tools was frustrating for some participants used to speaking when they were ready to. However, as participants gained more experience with the system they commented that such structuring brought more clarity and focus to their discussions, allowing a presenter to speak their entire train of thought and enabling those responding to ask more considered questions.

Throughout the series of sessions, various participants commented on the impact of the lack of non-verbal cues such as facial expressions and other body language. Some participants found that they listened more intently, a positive impact, and others found that the lack of non-verbal cues disrupted the flow of communications at times and took some time to become accustomed to.

The set of rules and resources, the nature of interaction and the technology and organisational structures that were enacted are presented within Table 23 below. This table presents the particular set of rules and resources associated with the organisational factor of Adaptable Communication Structures. When combined, these rules and resources provided the environment within which participants could translate aspects of face-to-face communication/meeting processes to the online sessions, and enact the Collaborative TiP that supported peer consulting. Over the series of six sessions, the collaborative technology structure was reinforced by participants as they repeatedly drew on these rules and resources to form a new practice of communicating online within peer consulting sessions. 


\begin{tabular}{|c|c|c|}
\hline & Element & Description \\
\hline & $\begin{array}{l}\text { Interpretive } \\
\text { Schemes }\end{array}$ & $\begin{array}{l}\text { Expectation that online sessions will follow a similar } \\
\text { format to face-to-face sessions } \\
\text { - Developing experience of how the online system } \\
\text { structures their communication } \\
\text { - Gaining experience of communication without non-verbal } \\
\text { cues }\end{array}$ \\
\hline $\begin{array}{l}\text { हे } \\
\frac{1}{2}\end{array}$ & Norms & $\begin{array}{l}\text { - Value knowledge sharing practices } \\
\text { - Value openness of communication } \\
\text { - Informal peer consulting performed } \\
\text { - Importance of reflection in groups }\end{array}$ \\
\hline & Facilities & $\begin{array}{l}\text { Features of the online collaboration system (particularly } \\
\text { the cueing feature) } \\
\text { - Basic PC hardware } \\
\text { - Internet connection (dial up and broadband) }\end{array}$ \\
\hline & Interaction & $\begin{array}{l}\text { Participants use features of the online system to structure their } \\
\text { communications within online peer consulting sessions }\end{array}$ \\
\hline$\frac{U}{3}$ & $\begin{array}{l}\text { Emergent } \\
\text { technology } \\
\text { structure(s) }\end{array}$ & $\begin{array}{l}\text { Aspects of face-to-face communications are translated online and } \\
\text { enable the enactment of the Collaborative TiP }\end{array}$ \\
\hline$\frac{3}{2}$ & $\begin{array}{l}\text { Other } \\
\text { organisational } \\
\text { structures }\end{array}$ & - Face-to-face meeting structures \\
\hline
\end{tabular}

Table 23: Elements of the Adaptable Communication Structures within AC

\section{Summary of the Collaborative Technology-in-Practice}

The evidence gathered and discussed here shows that participants at AC enacted the online system through a Collaborative-Use Practice at the Engaging stage. Within this stage, ten members of AC participated in six online discussions over a three month period. The prominent TiP that emerged within the participant group was one of collaborative use of the functions of email and the online collaboration system. Such use of the features of the online system was deemed collaborative, as groups of 
participants engaged in practice-related discussions within which they presented client cases, discussed case issues and developed and shared strategies for addressing those issues. Thus, discussions were undertaken by a particular group of participants within each session, and additional collaborative discussions occurred between members of the wider participant group following a session. This led to collaboration within AC on a number of levels, and collective understandings being reached across the organisation.

The participants who took part in the series of online sessions used various features of the online collaboration system to support the particular communications tasks that made up their discussions. An additional participant used the online collaboration system once, for discussion on specific management and operational issues. For reasons including the newness of the regional team, unfamiliarity with organisational processes, the urgency of other service provision issues and infrastructure difficulties these participants were not compelled to participate in the series of online peer consulting sessions.

Engaging with the online system did alter the system itself through the posting of session agenda, use of the presentation and browsing functions and the saving of the record of text entered during a session. However, the core of discussions was verbal exchange, which was not recorded or archived. Outcomes of consultation discussions did inform further systems use and contributed to their developing understanding of how online systems can support professional development, specifically peer consulting within AC.

The collaborative use of the online system by members of AC was influenced by a number of factors, the most prominent emerging as the Cooperative Culture of the organisation. The cooperative culture and participant's openness towards technology use had the most impact on their ongoing use of technology, as it allowed them to experiment with the key functions of the system, ask questions about the features that they were using and wanted to use in the future, while providing value through peer support. Elements of this factor were presented within Table 21. 
Additional factors that impacted on peer consulting transition included the Flexible Nature of AC and the Adaptable Communication Structures that staff typically engaged in. An overview of each of these factors was given and a summary of the key elements of each factor was given in Tables 22 and 23 respectively. These three prominent organisational factors and the elements associated with each combine to form the set of rules and resources of the Collaborative TiP. This technology structure and the nature of participant interaction are presented in Figure 16, below.

Within this Collaborative TiP there was ongoing interplay between elements of agency and structure, as depicted by the two way arrows within Figure 16. For example, as a particular set of rules as resources was enacted (see Table 23 above), participants drew on their knowledge and experience of the organisationally accepted ways of interacting within AC and carried aspects of their usual face-to-face communications into their online engagement within peer consulting sessions. As this occurred, the organisational factor of Adaptable Communication Structures within AC emerged and acted to support ongoing use of the collaboration system by influencing some of their assumptions, supporting the organisational values and norms and adding to their positive experience of online peer consulting. This ongoing interplay between elements of participants' actions (agency), the emergent organisational and technology structures and the nature of ongoing technology use within peer consulting session led to the enactment of the Collaborative TiP.

In summary, as members of AC interacted with features of the online system, particular rules and resources emerged that brought about the Collaborative TiP. This collaborative technology structure supported participants to continue through the series of online peer consulting sessions, and influenced their ongoing interactions with features of the online system. As they extended the peer consulting programme and advanced their use of the online system, they were influenced by a number of organisational factors the most prominent emerging as the Cooperative Culture of the organisation, the Flexible Nature of AC and the Adaptable Communication Structures participants typically engaged in. 


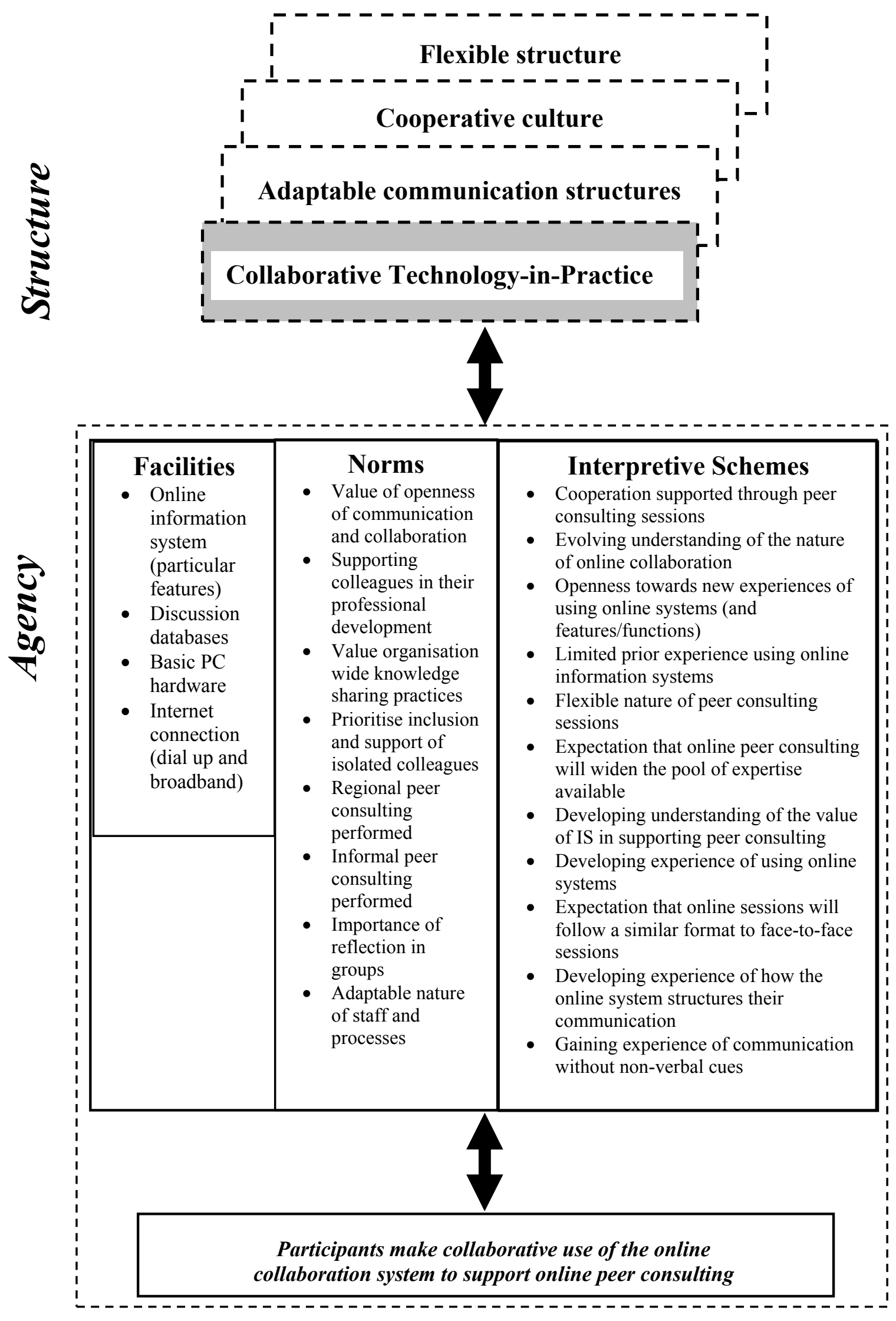

Figure 16: Collaborative Technology-in-Practice at the Engaging Stage within AC 
The next section describes the prominent TiP within CI and the key organisational factors that were observed to influence the nature of technology use, and ultimately impact peer consulting transition at this organisation.

\subsubsection{Technology-in-Practice at $\mathrm{Cl}$}

The focus of the Engaging stage at CI was participants' engagement in structured online peer consulting, through peer consulting session 1. Following on from the training sessions and informal use of the supporting system at the Experimenting stage, use of the online collaboration system at this stage was to be extended and refined through the session. At the Engaging stage, three of the 14 members of the participant group posted a message to the session forum, and seven participants accessed the forum and viewed their messages. This section describes the nature of their interaction with various technologies during this stage, and the impact of their actions on the session overall and on peer consulting transition.

In contrast to $\mathrm{AC}$, participants at $\mathrm{CI}$ enacted structures of technology that did not encourage but rather inhibited their progression through the stages of peer consulting transition. Applying the TiP framework to analyse elements of agency and structure revealed that participants within CI enacted the online system in a minimal and isolated manner at the Engaging stage. Therefore, I have termed the key outcome of technology use the Isolated Use Technology-in-Practice.

This isolated use of technology by participants was influenced by a number of factors, the most prominent emerging as the Regionally Focussed Structure of CI. This factor is described in detail, and the observed rules and resources are represented in Table 24. Two additional factors, the Individualistic Culture and the Institutionalised Meeting Structures of CI also influenced technology use. An overview of each of these factors is given, and the corresponding rules and resources associated with each are listed within Table 25 and 26. Finally, an overview of all elements of the Isolated Use 
Technology-in-Practice and a summary of the interplay between aspects of agency and structure is given.

\section{Regionally Focused Organisational Structure}

Chapter 6 described CI as a geographically distributed organisation that is structured regionally. This distributed nature has perpetuated a regional service provision focus at CI where typically one person is responsible for overseeing the staff and clients of one regional area. The distributed and regionally focused organisational structure has traditionally been viewed as an inhibiting factor to peer consulting, due to the lack of opportunities for staff to meet face-to-face. Within the Refining stage, this aspect of organisational structure emerged as factor that inhibited participants engaging with technology during the session.

The organisational structure of CI has led to an established organisational practice of participants working individually and regionally, largely in isolation of their peers. In keeping with this tradition and drawing on their past experience, each of the three participants that contributed to the online discussion forum did so in isolation of messages from other peers. The nature of the use of the discussion forum impacted the actions of others within session 1 , as one participant comments:

"what I initially did was log in and have a look and see what people were saying and I didn't find that it was being used very much at all, so I kind of let it go" (P2)

Other participants noted the value that they received from reading the messages of others, but felt that the nature of the work within their particular region was unique and any contribution they made to the discussion may not benefit others.

Indeed, interest in the individual and often unique practice of those in other regions was a motivating factor for some participants to access the online collaboration system and discussion forum, as P12 described: "we're both [P12 and P17] quite interested in getting more dialogue going with people who think differently". However, the emergent isolated-use practice acted to reinforce the regional 
differences to an extent that some participants did not see value in developing a collective approach to the session topic, and thus chose not to contribute to session 1. Although some level of individual knowledge and experience of these participants was shared with the seven participants who read the messages that were posted, the isolated-use practice acted to strengthen the sense of individual practice of each participant.

As participants contemplated participating in session 1 they drew on the regional service provision focus of $\mathrm{CI}$ as an inhibiting factor as this perpetuated significant time constrains for participants. Many participants were unable and/ or unwilling to spend time learning to use, or using a collaborative technology such as email and online discussion forums for peer consulting. This is mainly because their time was devoted to the day-to-day issues involved in supervising and supporting the staff in their region and providing services to their own clients. P15 captures the essence of this issue:

"I think there is something about the role that is very practical and either you are with your clients or you're supporting your [staff] or you're out in the community telling them about our work... I would say it's probably not in our job description to be reflecting on our practice with our peers regularly." (P15)

A small number of participants did give up their personal time for this, although not during the Refining stage and with no direct impact on session 1 (see Chapter 6, section 6.4). Nearly all participants indicated that time was an ongoing issue that influenced their use of online systems, including email. In the case of P12, having time to devote to online peer consulting, in comparison to other time-poor colleagues within other regions became an issue that impacted this participants involvement in session 1 and other, ad hoc online peer consulting:

"I was really keen and started a couple of threads and joined in threads until I got embarrassed by my over presence" (P12) 
Therefore, the emergent isolated use of the online collaboration system was a discouraging force for those who did contribute to session 1 , for those who access the session 1 forum and viewed the three messages posted and also for those who did not participate, as they were influenced by the actions of and discussion with other members of the participant group at CI.

In this recurrent $\mathrm{TiP}$, participants drew on aspects of agency, specifically rules including their knowledge and experience of CI institutional practices, in particular the regional service provision focus, and the perception that collaborative technology has limited benefit for supporting professional development. Within session 1, their individual and isolated use of the collaborative properties of the online system, as well as conference calls and email (see Chapter 6) reinforced these rules and led to the participant group enacting the Isolated Use TiP that failed to change their existing peer consulting practices, or elements of the online collaboration system itself (such as the forum and underlying databases). Orlikowski termed this recursive notion of technology the duality of technology, whereby technology is created and changed by human action at the same time that human action is mediated by existing technologies (Orlikowski, 1992).

In turn, the low level of technology use, especially the online discussion forum provided little value to those who actively participated in session 1 , or to the wider participant group. As the session went on, the lack of value received by participants from their Isolated-Use Practice acted to further reinforce their assumptions about the low degree of potential for technologies to be of benefit for peer consulting in the human services domain, as P12 stated, "it was a really extinguishing set of behaviours, it extinguished my contribution pretty effectively over the weeks". Also, the belief of many participants that the use of such technologies was not worth the cost (in time) of sacrificing either other work tasks or their personal time was upheld.

Through highlighting regional differences and reinforcing the individual practice and regional service provision focus at CI, the Isolated Use TiP enacted at the Refining stage thus supported and reinforced the Regionally Focused Organisational 
Structure and inhibited change within CI. The elements of this organisational factor that impacted on use of the system by participants and supported The Regionally Focussed Organisational Structure of CI and contributed towards the Isolated Use TiP at the Refining stage are included in Table 24 below.

\begin{tabular}{|c|c|c|}
\hline & Element & Description \\
\hline & $\begin{array}{l}\text { Interpretive } \\
\text { Schemes }\end{array}$ & $\begin{array}{l}\text { - Experience of system during function-based training } \\
\text { - Limited prior experience using technology for formal peer } \\
\text { consulting } \\
\text { - View that collaborative technology has limited benefit for } \\
\text { supporting professional development } \\
\text { - Developing understanding of value of collective approach to } \\
\text { issues }\end{array}$ \\
\hline & Norms & $\begin{array}{l}\text { - Regional staff and client focus } \\
\text { - Regions work largely in isolation of each other } \\
\text { - Staff and client needs prioritised over participant professional } \\
\text { development } \\
\text { - Acceptance of regional differences in practice }\end{array}$ \\
\hline & Facilities & $\begin{array}{l}\text { - Features of the online collaboration system (particularly the } \\
\text { discussion forum features) } \\
\text { - Basic PC hardware - accessibility an issue } \\
\text { - Internet connection (dial up and broadband) }\end{array}$ \\
\hline & Interaction & $\begin{array}{l}\text { The isolated use of the system highlighted regional differences in } \\
\text { practice, supports the regionally focused structure and leads to the } \\
\text { enactment of the Isolated Use TIP }\end{array}$ \\
\hline & $\begin{array}{l}\text { Emergent } \\
\text { technology } \\
\text { structure(s) }\end{array}$ & $\begin{array}{l}\text { Use of the system by few participants who posted separate messages } \\
\text { during session } 1 \text { leads to the enactment of the Isolated Use TiP }\end{array}$ \\
\hline 5 & $\begin{array}{l}\text { Other } \\
\text { organisational } \\
\text { structures }\end{array}$ & $\begin{array}{l}\text { - Regional service provision structure } \\
\text { - Distributed organisational structure }\end{array}$ \\
\hline
\end{tabular}

Table 24: Elements of the Regionally Focused Organisational Structure of CI 
In summary, the isolated use of the system by few members of the participant group at CI, and the Regionally Focused Organisational Structure combined with participant's low level of knowledge of and experience with online collaboration systems to inhibit their participation in peer consulting session 1. In turn, the isolated technology structure that began to emerge reinforced some participant's focus on regional service provision, the value placed in client focus and isolation of regions and peers.

The next two sections describe the organisational factors of the Individualistic Culture and the Institutionalised Meeting Structures of CI that contributed towards the Isolated Use TiP. As these factors had a lesser overall effect on technology use and the isolated technology structure that emerged at the Refining stage at CI, they are described in less detail than the Regionally Focused Organisational Structure. The key elements of the remaining two factors are outlined below, and the corresponding rules and resources associated with each are given in Tables 25 and 26 respectively.

\section{Regionally Focused Organisational Structure}

The distributed nature of CI has brought about the geographical isolation of each participant and the resultant regional service provision focus. Relatedly, the exact make up of the role of each participant differs due to the nature of the particular geographic region that they are responsible for, the community within which they are providing services and the number of staff that they manage (see Chapter 6 for a more detailed discussion). This regional focus sets the expectation that participants focus on the staff and clients within one area, and resulted in the organisational practice of participants working individually and regionally, largely in isolation of their peers.

Such elements of organisational structure and established working norms have seen an individualistic organisational culture emerge, that while enabling members of CI to effectively undertake their day to day work, was not supportive of cross-regional professional development. For example, within session 1 participants posted responses that detailed their experience and/ or perspective of the session topic, 
without responding to the messages of others. As stated above, some participants felt that aspects of their individual practice would not be of interest to others and chose not to contribute to the session discussion. Although some level of individual knowledge and experience of the participants who posted messages was shared with the seven participants who read the messages, the Isolated-Use Practice acted to strengthen the sense of individual practice of each participant, and therefore also the

\section{Individualistic Culture of CI.}

Adding to this, many participants have a low level of experience with or knowledge of using online collaboration systems, that was a contributing factor in the general scepticism towards the appropriateness and benefits of using technology as part of a formal and structured professional development programme observed within the participant group. As described below, participants preferred to use established methods of seeking and giving advice to their peers, such as face-to-face meetings and one-to-one telephone calls and email.

Although the training provided at the Structuring stage began to address such scepticism, it centred on the mechanical/ operational aspects of systems use and did not adequately cover the collaborative potential or value of the system (see Chapter 6 for additional discussion). Additionally, some participants experienced technical difficulties during the Engaging stage that added to their mistrust and scepticism towards the use of technology for professional development activities such as peer consulting. Drawing on the Individualistic Culture of CI, they then abandoned the session, and used already established peer networks to seek and give advice. This was reported to be nearly always on a one-to-one basis, which did not develop collaborative or collective understandings or knowledge of issues.

Although three isolated messages were posted and read by several participants, the message set did not greatly alter the technology, and therefore resources (functions of the online collaboration system) were minimally drawn on. Participants thus enacted these resources and particular elements of agency, including their assumptions, knowledge and organisational norms that did not modify their existing peer consulting practices, nor did they substantially alter the technology. 
In turn, the isolated technology structure that emerged influenced further use of technology by participants, discouraging others from engaging with the features of the system and posting a message. In summary, through the isolated use of technology participants previously held assumption that technology had limited value within the counselling domain, especially for formal professional development activities such as peer consulting was reinforced.

The key elements of this factor that were found to influence the Isolated Use TiP are presented within Table 25 below. This table shows that the particular knowledge and organisational norms within CI combined with aspects of the online collaboration system created a set of rules and resources that, when combined led to the Isolated Use TiP. As members of CI chose not to actively participate in peer consulting session 1, the Individualistic Culture of the organisation was reinforced, change to professional development practices did not occur and CI did not progress to the final stage of transition. 


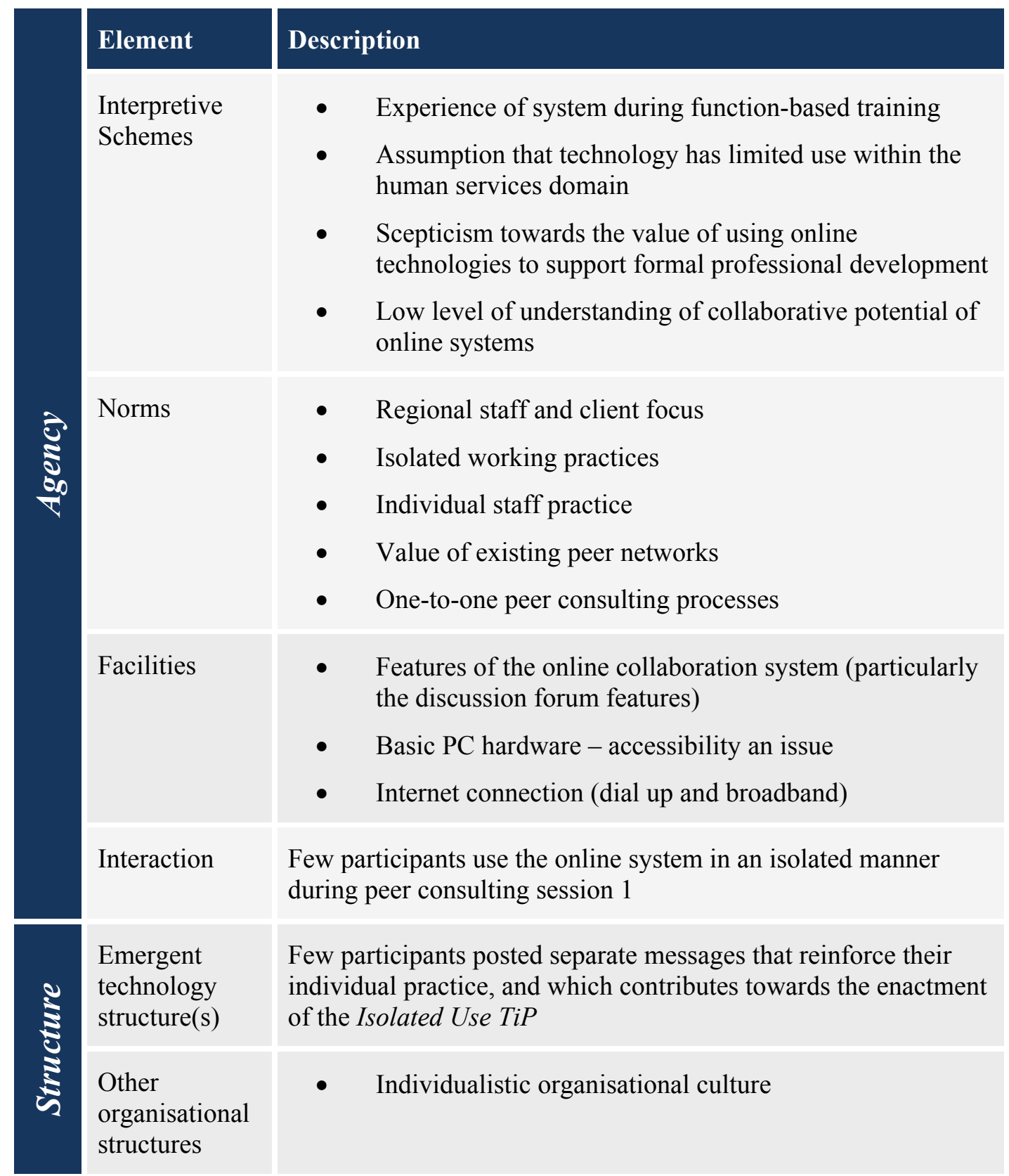

Table 25: Elements of the Individualistic Organisational Culture of CI

Combined with the two organisational factors already highlighted, one additional key factor, Institutionalised Meeting Structures at CI contributed to the emergence of the Isolated Use TiP, as described below. 


\section{Institutionalised meeting structures}

The Institutionalised Meeting Structures of CI strongly influenced participant's use of technology at the Refining stage. Most professional development within CI takes place within twice yearly, face-to-face national meetings where practice related information and experience is exchanged over a period of approximately four days with the goal of individual and collective staff professional development. Participants were generally satisfied with the outcomes of these meetings, and often 'parked' issues to be raised and discussed within them.

This third key factor influencing the use of online collaboration system at the Refining stage centres on participants' preferences for face-to-face interaction with clients, the staff that they manage and their colleagues both within and external to CI. The nature of the work that participants and other members of CI undertake is based on face-to-face engagement, usually on a one-to-one or less frequently one-to-many basis. This is due to the Regionally Focussed Structure, as outlined above, and also because of the personal and sensitive nature of the work of members of CI.

Due to this inherent sensitivity, the majority of practice related issues that participants identified as appropriate and valuable for discussion through peer consulting session 1 are also of a highly sensitive nature. Therefore, the type of information exchanged, or needing to be exchanged via the discussion forum had an impact on systems use by participants. Many participants held the perception that there was a high chance of the meaning of a message being misinterpreted, and that when posting a message they would not be able to immediately assess whether a message recipient had correctly interpreted the intended meaning. Therefore, many members of the group were apprehensive about engaging in asynchronous, text-based discussions on issues of practice.

Such apprehension acted to influence their actions within session 1, many participants preferring to read the three messages posted but not contribute a message themselves. As there were no direct replies to any messages on the session forum, the participants who had posted them were unsure if others had read and understood them, and were 
discouraged from posting further messages during the period of session 1 . Thus, the apprehension towards technology-supported interactions was a factor that led to participants enacting the Isolated Use TiP. In turn, the Isolated-Use Practice added to participants' apprehensions, and thus also acted to reinforce their preference for face-to-face engagement, a key organisational norm.

The preference for face-to-face interactions, combined with the lack of experience with and knowledge of collaborative technologies acted to reinforce the notions of participants that such use of technology within the counselling domain was inappropriate. Ultimately, these factors resulted in the isolated use of technology for peer consulting. The key elements of this factor that were found to influence the Isolated Use TiP are presented within Table 26 below.

This table illustrates that participants drew heavily on the organisational norms related to their practice and entrenched organisational and individual processes to enact a technology structure that supported established meeting structures and the value that such structures provided. Thus, through the minimal use of the online collaboration system in a way that reinforced the status quo at CI existing practices were not changed, and the Isolated Use TiP was enacted. 


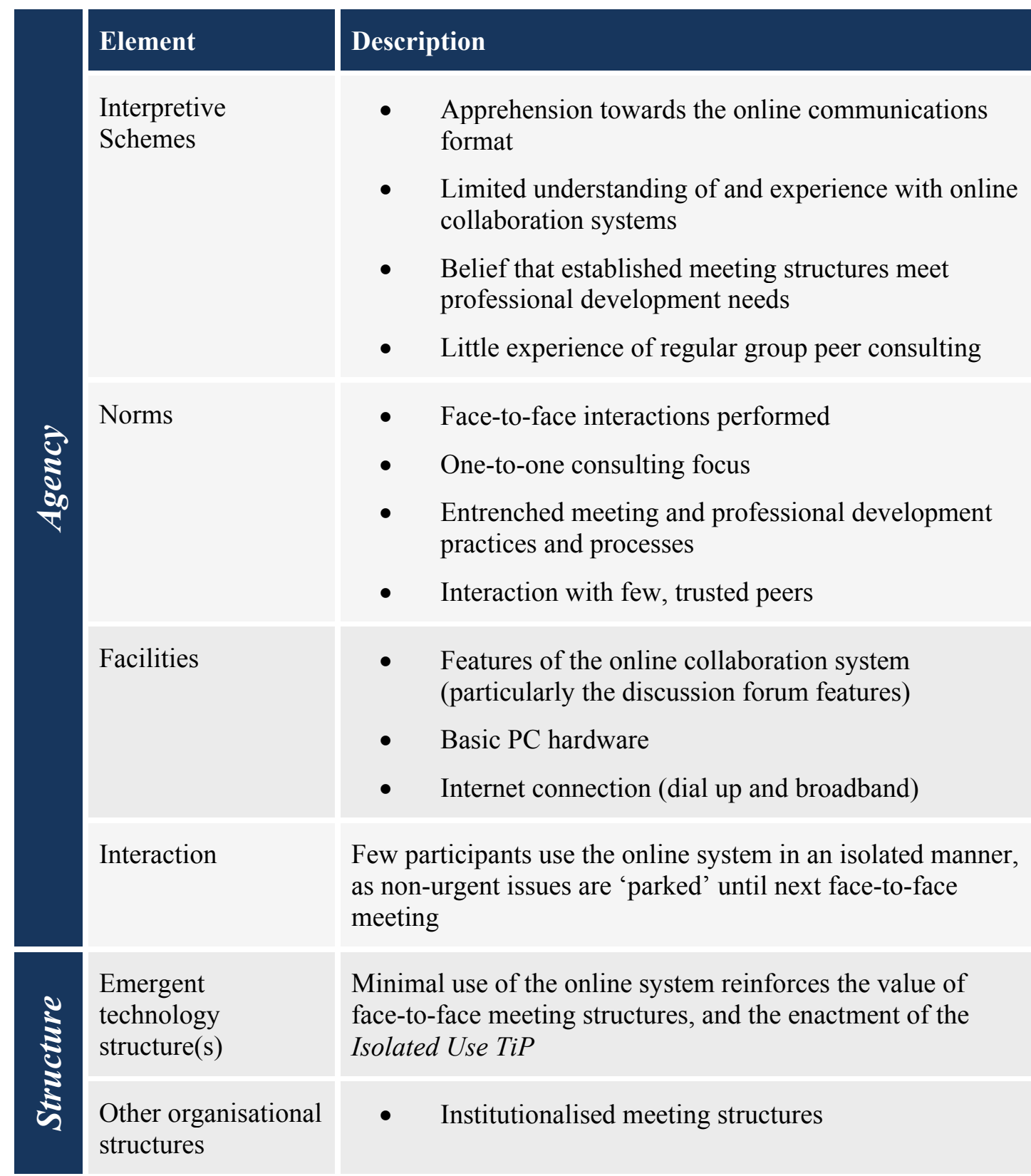

Table 26: Elements of the Institutionalised Meeting Structures of CI

\section{Summary of the Isolated Use Technology-in-Practice}

The discussion above shows that the Isolated Use TiP was most prominent within the participant group at CI at the Refining stage. I have deemed the nature of their technology use isolated as the three participants who took part in the online discussion accessed the discussion forum to read each others messages and post one reply each, resulting in a total of three isolated messages that constituted discussion forum use. 
Four additional participants entered the discussion forum to view messages, but were not compelled to contribute a message. The remaining members of the participant group chose not to participate in session 1 at all, brining another level of isolation into play.

While seven participants accessed the peer consulting session 1 discussion forum, only three messages were posted, that did not form the type of collaborative discussion that was evident during the Experimenting stage. Thus, while some degree of practice-related knowledge and experience sharing occurred, the level of discussion needed for peer consulting to occur was not reached. Thus, messages were not shared within the wider group which resulted in isolated messages and understandings.

The general lack of experience that participants had in using collaborative technologies, especially for professional development activities, their preference for face-to-face engagement with peers and the organisations regionally orientated and isolating structure influenced the use of technology during session 1. Many participants saw the use of technology, especially online discussion forums, as countercultural and incompatible with their preferred and established ways of working and engaging in professional development activities. This limited use of technology acted to reinforce the norms of face-to-face interaction and working in isolation of others, whist also supporting the regional service provision focus of the organisation and participants' previously held assumptions about the appropriateness of technology within their practice domain.

While some members of CI enacted more participatory, or collaborative TiPs, the prominent TiP that emerged within the participant group was one of isolated use of the functions of email, conference calls and most importantly the online collaboration system. Engaging with the online collaboration system in this manner brought about minimal changes to the system itself and no significant or long-lasting change to peer consulting processes at $\mathrm{CI}$. 
The isolated use of technology by participants was influenced by a number of factors, the most prominent emerging as the Regionally Focussed Structure of CI. This factor was described in detail, and the observed rules and resources represented in Table 24. Two additional factors, the Individualistic Culture and the Institutionalised Meeting Structures of CI also influenced technology use. An overview of each of these factors was given, and the corresponding elements of each were listed within Tables 25 and 26 respectively.

These three prominent organisational factors and the elements associated with each combine to form the set of rules and resources of the Isolated Use TiP, as illustrated in Figure 17. 


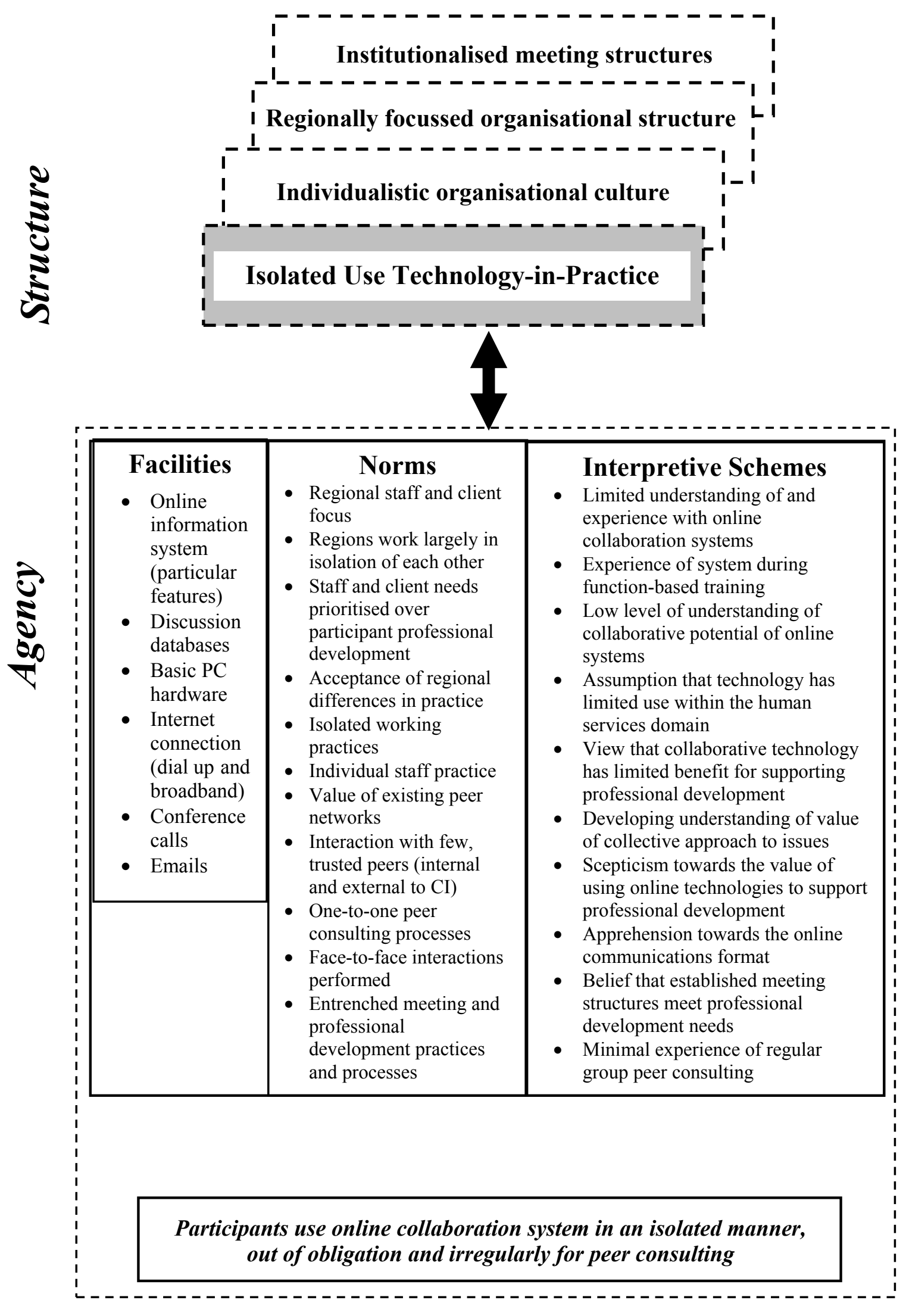

Figure 17: Isolated Use Technology-in-Practice at the Refining Stage within CI 
The arrows in Figure 17 depict the ongoing interplay between elements of agency and structure within the Isolated Use TiP. For example, in the emergence of the Individualistic Organisational Culture of CI (see Table 25) participants constantly drew on their experiences, assumptions and expectations of the use and value of online collaboration systems for supporting group professional development. As they drew on these rules, they were influenced by the organisational norms of working in isolation from peers and focusing on regional and individual needs and practice. Correspondingly, the nature of their use of features of the online collaboration system was minimal and isolated.

As the duration of Session 1 progressed, the minimal use of the discussion forum inhibited further use of the system by members of the participant group, and they turned to traditional, one-to-one peer consulting with their trusted colleagues within existing peer networks.

Thus, the organisational factor of the Individualistic Culture of CI influenced participant actions, and in turn the emergent isolated technology structure that emerged reinforced participant assumptions, strengthened the current ways if working and interacting at $\mathrm{CI}$ and inhibited further systems use by members of the participant group. Resultantly, the Isolated Use Technology-in-Practice discouraged participants from continuing with online peer consulting sessions, and contributed to CI halting the progress of peer consulting transition at the Refining stage.

In Section 7.4, I provide a reflection on the outcomes of applying the Technology-inPractice framework, particularly the various organisational factors identified at CI and $\mathrm{AC}$ that impacted online professional development sessions. 


\subsection{Technology-in-Practice Summary: Organisational Factors Revealed}

Within Chapter 7 I have described certain aspects of the ongoing use of technology by members of AC and CI at the Engaging stage of peer consulting transition.

Application of the Technology-in-Practice framework enabled an in-depth analysis of the interaction of groups of participants with particular features of the online systems, and revealed the various rules and resources, or knowledge of work and ways of undertaking work, and the facilities necessary to accomplish such work that were found to be at play within each organisation (see Figures 16 and 17 for an overview). Through this analysis I drew out a significant finding of this research: the particular set of rules and resources that emerged as most influential at AC and CI, which I identified as the predominant Technology-in-Practice. As described above, AC enacted the Collaborative TiP, and at CI the Isolated Use TiP was most prominent.

While these findings illuminated the nature of the technological artefacts or the key features and functions of the online systems, they also highlighted the importance of the actions of individuals, their agency, and the social context within which these elements interacted as technology was used to support online professional development (Dery, et al., 2006).

The second key set of findings of this chapter relate to the social and organisational structures that emerged from participants' interactions with features of the online systems, which acted to influence their ongoing systems use. This analysis answered the call of researchers such as DeSanctis and Poole (1994), Orlikowkski (2000) and Dery et al. (2006) as I gave particular emphasis to the 'other organisational structures', or organisational factors, present within each organisation that impacted technology use and ultimately also Peer Consulting Transition. At AC, the organisational factors of their Adaptable Communication Structures, the Flexible Nature of the organisation and most prominently their Cooperative Culture supported them in progressing to the final stage of transition. In contrast, CI experienced organisational factors of the Individualistic Culture, the Regionally Focussed Structures of the nationally distributed CI offices, and the Institutionalised Meeting Structures though 
which professional development historically took place. These factors were instrumental in prematurely suspending their progress through the model at the Engaging stage.

Additional findings, including the influence of the actions of key persons at $\mathrm{AC}$ and CI on the Technologies-in-Practice enacted, and a comparison of the Collaborative TiP of AC and the Isolated Use TiP of CI is given as part of the Chapter 8 discussion. 


\section{Discussion, Implications and Conclusion}

\subsection{Chapter Overview}

In this study I have worked with two resource poor, distributed social services organisations, AC and CI. The staff of these organisations had experienced differing levels of personal and professional isolation and realised the need for a higher level of connectedness with their peers. Also, key members of each organisation had identified online ICT as a possible means to support and enable additional professional development opportunities and had some awareness of the potential value this might offer individuals, peer groups, and the wider organisations. Despite such awareness, inhibiting factors such as organisational infrastructure, available resources, the knowledge and experience of AC and CI staff, and their commitment to service provision prevented them from addressing staff isolation and finding ways to offer increased opportunities for them to engage in regular and ongoing peer-based professional development.

Through this research I sought to introduce ICTs and professional development processes, specifically peer consulting, to relive the isolation of senior staff at AC and CI and enhance organisation-wide professional development, and also to extend the body of knowledge on how organisations transition towards complimenting traditional professional development activities with an online programme. To pursue these research objectives I gathered data from various sources, most importantly participant interviews and my own observations and employed qualitative analysis techniques to generate the reported research results.

Within this final chapter I summarise the main research results and other important findings, and discuss some implications for ongoing research, participant organisations, and other practitioners. Lastly, I conclude the thesis with some final comments. 


\subsection{Overview of Findings}

Exploring peer consulting transition at $\mathrm{AC}$ and $\mathrm{CI}$ involved intense periods of collaborative activity through which participants and I undertook particular actions, introduced ICT and peer consulting processes and evaluated the outcomes and resulting learning of each action. Alongside this, I carried out ongoing research activities to capture and analyse the journey of each organisation and the individual and collective experiences of participants. Taken together, the 'action' and 'research' components of the study provide a rich description of the pathway that each participant organisation took towards introducing an online peer consulting programme, presented in Chapter 4 as the Model of Peer Consulting Transition. This model comprises the five stages of Defining, Structuring, Experimenting, Engaging and Embedding and associated activities.

Not surprisingly each organisation experienced peer consulting transition in unique, although still comparable, ways and reached different outcomes. My analysis of the particular activities and decisions that participants at $\mathrm{AC}$ and $\mathrm{CI}$ undertook and the subsequent results and key findings are described in Chapters 5 and 6 (respectively).

A more in-depth investigation of the nature of the participants' use of online ICT to support their peer consulting activities enabled me to unearth several organisational factors that greatly influenced their movement through the transition process. Chapter 7 describes how I applied the Technology-in-Practice (TiP) framework to highlight the organisational factors at work within AC and CI at each stage of transition, and to show how such factors came about through a process of ongoing structuring. Basically, my TiP analysis described the interplay between elements of agency (the actions of participants) and structure (the emergent technology and other social structures) and revealed particular organisational factors and their impact on peer consulting transition at $\mathrm{AC}$ and $\mathrm{CI}$.

The following section outlines how I have addressed the research questions that guided this study and led to the main research findings. 


\subsection{Addressing the Research Questions}

In response to the situations faced by AC and CI, I undertook an action research study and worked collaboratively with both organisations on the development, initiation and evaluation of online peer consulting programmes. This study was guided by the primary research question:

“How do NZ social services organisations apply online technologies to enhance the professional development of their staff?"

I have answered this research question in two distinct yet interconnected ways:

1. Developing the Model of Peer Consulting Transition to explain each organisations journey towards introducing an online peer consulting programme

2. Applying the Technology-in-Practice framework to reveal the prominent organisational factors that brought about particular outcomes for each organisation

Within Chapter 7 I have described in detail the application of the TiP framework at the main action stage of the model, the Engaging stage, and identified key aspects of the nature of participant's use of ICT for online peer consulting in learning communities. This description therefore addresses the secondary research question:

"Within communities of practice, what is the nature of the use of online technologies for professional development activities?"

By combining the Model of Peer Consulting Transition with an in-depth explanation of the social and technological structure that impacted programme development, I have detailed the path that each organisation took to applying online ICTs to enhance the professional development of their staff, including inhibiting and encouraging organisational factors, and thus answered the primary research question. I summarise these two complimentary findings of the study below. 


\subsubsection{Model of Peer Consulting Transition}

While other models exist that depict various forming, functioning and ending stages of CoPs (see for example Wenger, 1998; McDermott, 2000; Gongla \& Rizzuto, 2001) and more recently online communities (Hildreth \& Kimble, 2000; Lee 2004), none of these models map the particular activities and decisions that need to be addressed during the establishment of an online professional development programme within distributed community organisations.

Conducting an action research study in which two participant organisations went through the process of complimenting their existing professional development practices with an online peer consulting programme enabled me to map this transition. Specifically, I gathered data throughout all research phases (mainly through interviews, observation and organisational documentation) and employed various data organisation and coding techniques to develop categories and data sets, identify themes within and across categories, and develop memos that explained the nature of each category and its links to other categories. My analysis resulted in the main finding of this study, the Model of Peer Consulting Transition (see Chapter 4). This model consists of five stages each made distinct by a particular combination of key activities and a Trigger Point, the catalyst for a learning community (in this research, a peer consulting group) moving to the next transition stage. Each stage builds on the outcomes (including new knowledge and experience) of previous stage(s) and naturally occurs over a period of time but, unlike many other development models, the transition model allows for an organisation to return to the activities of earlier stages when necessary, and then either continue progression through remaining stages or conclude their transition.

The model was generalised from the particular stages and various observed actions that participants at AC and CI undertook during the study (see Chapters 5 and 6 respectively). As such, the unique journey of each organisation gave rise to some important differences between the stages, activities, trigger points and outcomes as depicted in Table 27 below. Several points of this comparison are of most interest: 
- Despite differences between AC and CI (such as organisational development levels, staff numbers, geographical distribution, key drivers and elements of organisational culture), the observed actions of participants could be grouped within similar categories

- Although some activities were undertaken differently within each organisation, the sequence of their actions was comparable over the first four stages of transition

- Differences in elements of organisational structure, culture, and development level affected peer consulting transition in different ways, at different stages. Particular elements are discussed further below, as organisational factors (social and technology structures) of TiPs

- Key participants at each organisation exhibited leadership behaviours that greatly influenced attitudes and actions of other participants, and subsequently also the overall transition effort (see below for further explanation)

- Within each organisation there were some prominent factors that, at times, encouraged AC and CI's movement through the stages of Peer Consulting Transition, and sometimes inhibited progress. I applied the TiP framework to elucidate and analyse such factors, as next summarised. 


\begin{tabular}{|c|c|c|c|c|c|}
\hline $\begin{array}{l}\text { Transition } \\
\text { Stages }\end{array}$ & Stage 1 & Stage 2 & Stage 3 & Stage 4 & Stage 5 \\
\hline $\begin{array}{l}\text { Generalised } \\
\text { Model } \\
\text { (Chapter 4) }\end{array}$ & $\begin{array}{l}\text { Defining Stage: } \\
\text { - Mapping current activities } \\
\text { - Documenting current ICT } \\
\text { support } \\
\text { - Developing the concept }\end{array}$ & $\begin{array}{l}\text { Structuring Stage: } \\
\text { - Planning far a series of } \\
\text { sessions } \\
\text { - Selecting and setting up } \\
\text { systems } \\
\text { - Training on ICT use }\end{array}$ & $\begin{array}{l}\text { Experimenting Stage: } \\
\text { - Participating in informal online } \\
\text { peer consulting } \\
\text { - Altering the plan for sessions } \\
\text { - Reconfiguring systems }\end{array}$ & $\begin{array}{l}\text { Engaging Stage: } \\
\text { - Participating in peer } \\
\text { consulting sessions } \\
\text { - Formalising peer consulting } \\
\text { sessions } \\
\text { - Refining systems set up }\end{array}$ & $\begin{array}{l}\text { Embedding Stage: } \\
\text { - Cementing the online peer } \\
\text { consulting concept } \\
\text { - Advancing technical } \\
\text { infrastructure }\end{array}$ \\
\hline $\begin{array}{l}\text { AC's Instance } \\
\text { of Model } \\
\text { (Chapter 5) }\end{array}$ & $\begin{array}{l}\text { Scoping Stage: } \\
\text { - Developing concept } \\
\text { - Discovering ICT } \\
\text { - Determining activities }\end{array}$ & $\begin{array}{l}\text { Shaping Stage: } \\
\text { - Honing concept } \\
\text { - Selecting/setting up } \\
\text { systems }\end{array}$ & $\begin{array}{l}\text { Familiarising Stage: } \\
\text { - Training in action } \\
\text { - Planning sessions }\end{array}$ & $\begin{array}{l}\text { Engaging Stage: } \\
\text { - Reviewing IS use } \\
\text { - Reforming Sessions } \\
\text { - Performing online } \\
\text { consulting }\end{array}$ & $\begin{array}{l}\text { Embedding Stage: } \\
\text { - Advancing infrastructure } \\
\text { - Cementing the Concept }\end{array}$ \\
\hline $\begin{array}{l}\text { Cls Instance } \\
\text { of Model } \\
\text { (Chapter 6) }\end{array}$ & $\begin{array}{l}\text { Defining Stage: } \\
\text { - Documenting ICT Support } \\
\text { - Mapping activities } \\
\text { - Developing concept }\end{array}$ & $\begin{array}{l}\text { Structuring Stage: } \\
\text { - Training } \\
\text { - Selecting/setting up } \\
\text { systems } \\
\text { - Planning sessions }\end{array}$ & $\begin{array}{l}\text { Experimenting Stage: } \\
\text { - Restructuring plan } \\
\text { - Actioning sessions }\end{array}$ & $\begin{array}{l}\text { Refining Stage: } \\
\text { - Reconfiguring systems } \\
\text { - Reforming sessions } \\
\text { - Performing online } \\
\text { consulting }\end{array}$ & (CI did not reach this stage) \\
\hline $\begin{array}{l}\text { Desired } \\
\text { Outcomes }\end{array}$ & $\begin{array}{l}\text { Understanding is gained of } \\
\text { current key peer consulting } \\
\text { practices and ICT use, an } \\
\text { initial peer consulting concept } \\
\text { is developed. The potential of } \\
\text { online peer consulting is } \\
\text { apparent. }\end{array}$ & $\begin{array}{l}\text { The organisation is } \\
\text { prepared to engage in } \\
\text { online peer consulting by } \\
\text { planning for sessions and } \\
\text { introducing ICT. Interest is } \\
\text { sparked and motivation } \\
\text { raised. }\end{array}$ & $\begin{array}{l}\text { The organisation is ready to } \\
\text { engaged in formal sessions by } \\
\text { experiencing initial online peer } \\
\text { consulting, revising session plan } \\
\text { and setting up supporting ICT }\end{array}$ & $\begin{array}{l}\text { Following a series of peer } \\
\text { consulting session, } \\
\text { participant actions, session } \\
\text { structure and online ICT } \\
\text { combine to provide an ideal } \\
\text { environment }\end{array}$ & $\begin{array}{l}\text { Online peer consulting is } \\
\text { accepted and integrated with } \\
\text { other organisational } \\
\text { professional development } \\
\text { activities }\end{array}$ \\
\hline
\end{tabular}

Table 27: Components of Transition Models 


\subsubsection{Application of the TiP Framework}

After providing a description of the pathway of each organisation through peer consulting transition, I extended the model by applying the Technology-in-Practice (TiP) framework to explore the organisational factors at work at each stage of transition, and describe how they came about through a process of ongoing structuring (see Chapter 7 for a full discussion). This analysis described the interplay between agency (the actions of participants) and structure (the emergent technology and other social structures).

Comparing the cases of $\mathrm{AC}$ and $\mathrm{CI}$ shows two quite different technology structures emerging; at $\mathrm{AC}$, the Collaborative TiP emerged, whereas CI participants enacted the Isolated Use TiP (see sections 7.3.1 and 7.3.2 respectively). Table 28 provides a summary comparison of key elements of the prominent TiPs at AC and CI. Reviewing the components of agency, the TiPs at AC and CI shared some similar resources, namely the functions of ICT used to support peer consulting sessions (and also training and familiarisation periods). However, many differences emerged in the underlying organisational values and practices, as well as the assumptions, knowledge and experience of individual participants (listed as 'rules' in Table 28). It is these differences that had a greater cumulative impact on the nature of participants' interactions with ICTs, and ultimately brought about enacted TiPs.

I identified a number of emergent factors within each organisation that impacted further ICT use, influenced their actions and affected AC and CI's progress through the stages of the model. Specifically, AC's most influential organisational factors at the Engaging stage included:

- The cooperative organisational culture

- Distributed and flexible nature of AC

- Adaptable communication structures

These factors encouraged members of AC to actively use the ICT for online peer consulting, and explore ways to make better use of the functionality. As a result, AC 
held a series of active online peer consulting sessions, met desired outcomes and progressed through all stages of Peer Consulting Transition to reach the final stage of Embedding.

The key organisational factors that emerged at CI were:

- The regionally focused structure

- The individualistic organisational culture

- Pre-existing meeting structures

In contrast to what I observed within AC, these factors impeded the path of CI through the stages of the model and acted to reinforce existing ways of interacting with peers, and ultimately their progress arrested at the fourth transition stage. Consequently, CI did not reach the final Embedding stage of transition and no lasting effect was made to their professional development practices.

I draw parallels between these elements of AC and CI's organisational structure and culture and the operational (and to a lesser extent individual) level factors described by Dery et al. (2006). I propose that these factors could be potential indicators of organisational readiness for transitioning to online peer consulting, particularly in light of the nature of their influence at peer consulting sessions of the Engaging stage, namely:

- AC: participants make collaborative use of online ICTs to support online peer consulting

- CI: participants use online ICTs in an isolated manner, out of obligation and irregularly for peer consulting 


\section{Collaborative TiP at AC}

- Adaptable communications structures

\section{Organisational}

Factors:

Rules:

\section{Resources:}

- Flexible structure

- Cooperative culture

- Value openness of communication and collaboration

- Support colleagues' professional development

- Open to new experiences with ICTs

- Limited prior experience of online ICTs

- Expect similar format for online and face-to-face sessions

- Value organisation-wide knowledge sharing practices

- Expect online peer consulting will widen expertise poo

- Prioritise inclusion of isolated colleagues

- Cooperation supported through online peer consulting sessions

- Adaptable nature of staff and processes

- Flexible nature of sessions

- Features of online ICT:

- VOIP

- Instant messaging

- Web browsing

- Discussion forums and databases*

- File repository*

- Announcement function*

- Basic PC hardware

- Internet connection (dial up and broadband)

- Email

*minimal use of this ICT

\section{Isolated Use TiP at CI}

- Institutionalised meeting structures

- Regionally focussed structure

- Individualistic culture

- Value existing peer networks and few, trusted peers

- Regions work largely in isolation of each other

- Apprehensive towards online communications

- Scepticism towards value of ICT for PD \& in their industry

- Limited prior experience of online ICTs

- Experience of online ICT during training sessions

- Prioritise regional staff and client needs (over PD)

- Minimal experience of regular group consulting

- Growing understanding of value of group approach to issues

- Entrenched meeting and PD practices and processes

- Belief that established meeting structures meet PD needs

- Features of online ICT:

- Discussion forums

- Discussion databases

- File repository*

- Announcement function

- Participant profiles

- Basic PC hardware

- Internet connection (dial up and broadband)

- Conference calls

- Email

*minimal use of this ICT

Table 28: Overview of Key Elements of TiPs at AC and CI 


\subsection{Implications for Research}

In examining the main research question of this study (see section 8.2) I have intervened in actual organisational settings to address a particular issue of professional isolation by introducing online peer consulting processes and supporting ICT. Through careful collection and analysis of data I have generated two key research findings as well as gleaning other learning that contributes to academic knowledge and is of value to the wider academic community through raising several opportunities for further research.

This research makes several key contributions to current academic knowledge and the research community. I have focused this research on two NZ human services organisations. These organisations face unique conditions, including operating in main centres as well as isolated communities, needing to maintain professional standards with a changing and part time workforce and being traditionally 'resource poor'. In NZ, many service providers have been grappling with the financial pressures of investing in the areas of information technology and staff professional development (MSD, 2001). The need to invest in professional development has been spurred on by the need to improve the effectiveness of organisations in managing the staff who deliver social services to communities (MSD, 2001).

Although this need was realised several years ago, and the social and community aspects of continued adult learning and professional development are coming to the fore, there exists little research that addresses the introduction of professional development initiatives within NZ human services organisations. By exploring the conditions that affected two such organisations when introducing online peer consulting I have shed some light on the particular structure, culture and needs of these organisations and added to this growing body of knowledge. Specific implications related to the model generated through this study, the use of the TiP framework, the role of organisational leaders in the transition process, the use of MST, and the Distance Action Research approach are outlined below. 


\subsubsection{Generating the Model of Peer Consulting Transition}

As organisations strive to offer improved services to the communities within which they operate, researchers including Gittell et al. (2000), Brown (2001) and Denison et al. (2002) have highlighted the importance of providing additional information and communications channels for staff in order to facilitate information and knowledge sharing. Peer consulting is one way that such information and knowledge sharing typically occurs within organisations, and this study addressed online alternatives to traditional peer consulting activities and events. Specifically, the model that I have generated and its particular stages and activities offers enhanced understanding of the process that an organisation goes through when complimenting traditional professional development activities and channels with online alternatives. By mapping out the experience of two NZ social services organisations, this research offers important insights into the challenges faced by organisations that attempt to introduce an online peer consulting programme, and identifies some elements of organisational readiness previously unknown.

Researchers now have the opportunity to apply the Model of Peer Consulting Transition and assess the validity of the five stages and subsequent activities within other research settings. For example, it would be interesting to gather results from organisations of a different size, domain, and structure and compare them to the findings reported within this thesis. In addition, applying the model to an organisation outside of NZ may yield variations in the stages and/or activities.

There is also the possibility of adapting the model to make it relevant to other professional development processes, in addition to peer consulting. There are myriad activities, events and processes that an organisation may use to guide the professional development of their staff. The particular set of professional development offerings of an organisation are likely to change over time, and therefore a model to guide the development and introduction of other professional development processes would be beneficial. 


\subsubsection{Applying the Technology-in-Practice framework}

I have made a key contribution to research through applying the TiP framework (Orlikowski, 2000) within stages of the model to surface and analyse the organisational factors that influenced ICT use and affected the development of the online peer consulting programme within each organisation. Recently, Dery et al. (2006) called for further adaptation of the framework to promote the importance of the relationship between technologies-in-practice and other structures. In answer to this call I have demonstrated that the framework, as Orlikowski intended, provides a valuable lens through which to analyse the interaction between elements of agency and structure, and also offers the organisational researcher a way to draw out and explain the impact of other structures, that I have termed organisational factors. Researchers are now challenged to continue to apply the TiP framework in this manner, and perhaps develop further adaptations that are particularly effective in uncovering and explaining organisational, contextual or situational factors at work within organisations.

The Collaborative and Isolated TiPs at AC and CI (respectively) resulted from my TiP analysis highlighted the importance of the nature of ICTs selected to support peer consulting (in this study, the Blackboard and Chatterbox systems). At the time of the Structuring and Experimenting stage activities at both organisations (late 2004 to 2005) what has come to be known as Web 2.0 technologies such as social networking software were not readily available or used within NZ organisations. For example, the popular social networking site Facebook and the peer networking tools within it were launched in 2004, and available to the public from 2006 (Facebook factsheet, 2010). By the time participant organisations entered the Engaging stage (late 2005 to 2006) I deemed it problematic to switch to other ICTs, as this would likely have significantly disrupted the study by reversing the significant investments in time and thinking spent in the selection, set up, training and trialling of Blackboard and Chatterbox. 
Although the nature of ICT applied in this study could be deemed a technical limitation, there is now an opportunity for further research into the process of transitioning peer consulting with different supporting online ICTs. Researchers could build on this study by replicating the transition to online peer consulting using different supporting ICTs, such as the collaboration tools: peer-to-peer instant messaging and audio/video chat, blogs, wikis and customisable website home pages. Applying the transition model and analysing participant engagement with other supporting online ICT is likely to reveal a wider pool of elements of agency and structure (i.e. technology and other organisational structures) that impact transition towards, and overall success of, an online peer consulting programme. A greater understanding of the range and nature of agency and structure factors that combine to either propel or arrest an organisation's progress through a process of peer consulting transition will add more depth to academic knowledge in this area.

Although the TiP analysis led to valuable insights and findings in this study, I acknowledge that other models or frameworks may also offer explanations for why $\mathrm{AC}$ and $\mathrm{CI}$ experienced different outcomes. For example, the Technology Acceptance Model (TAM) could be used to draw out the factors that influenced participants' attitudes towards using online ICTs, and therefore also likely impacted their behaviours (Adams, Nelson \& Todd, 1992). Applying a model such as TAM to the early stages of the Model of Peer Consulting Transition would have revealed important insights about participants' assumptions and beliefs of the value of online ICTs in supporting professional development. Also, any apprehension or confidence they felt towards upcoming ICT use at the Experimenting and/or Engaging stages would be uncovered.

Within this study, my aim was to explore the introduction and use of online ICTs as participants engaged in online peer consulting sessions. Therefore, a dynamic model such as Orlikowski's TiP framework enabled the analysis of ongoing and situated use of ICTs over time. This revealed the nature and means by which organisational factors (including technology and social structures) emerged from participant action. A model such as TAM could not have been applied in this way, nor led to the 
findings presented in Chapter 7. The use of alternative models to examine elements of ICT use at stages of the Model of Peer Consulting Transition now provides researchers with the opportunity to extend the findings of this study.

\subsubsection{Applying Media Synchronicity Theory}

Earlier in this study I applied Media Synchronicity Theory (MST) to identify the communications processes present (specifically conveyance and convergence) at AC and CI, and the points at which ICTs could add most value to their professional development processes. As evident in Chapter 5, the particular ICTs and combination of technology tools suggested by MST (including email, discussion forums, audio conferencing) successfully supported AC's online peer consulting activities. On analysis, I determined that certain traits of these ICTs were aligned with aspects of AC's culture, such as flexibility, inclusiveness and adaptability. In contrast, elements of CI's preferred ways of working and organisational structure and culture were not supported by their use of ICT for online peer consulting, and their consulting programme was unsuccessful.

The dissimilarity in outcomes at AC and CI does not indicate that MST was, as a theory, more or less helpful or 'correct' in selecting ICTs to support peer consulting. Instead, MST was a valuable analysis tool applied in this study and I believe that the ICTs most appropriate for each organisation were identified, selected and applied. The reason that $\mathrm{AC}$ progressed through the main action stage of peer consulting transition and CI moved back to an earlier stage was most prominently influenced by organisational factors, as revealed through the TiP analysis and results (see 8.2.2 above or a fuller discussion in Chapter 7).

Reflecting on my use of MST and the results achieved, I have made an important contribution to research knowledge as few studies have successfully applied this theory and extended the recent academic literature in the area of media selection and application and synchronicity. Further value could now be derived by applying MST 
at various stages of the Model of Peer Consulting Transition, for example at the Engaging stage MST could be used as an analysis tool to unearth the nature of the particular communicative actions and processes of a peer consulting group, and whether they used the most effective features/functions of the available ICTs. The findings could then influence participants' interactions with particular features of ICT within subsequent online peer consulting sessions, and perhaps improve their communication and peer consulting experience and subsequently also the individual, group and organisational value derived from professional development.

\subsubsection{Highlighting leadership roles}

There is one additional contribution related to the Model of Peer Consulting Transition and my use of the TiP framework in this study, related to the role of key persons. Rogers (1995) classified behavioural characteristics, such as the role of change agents as one of the three main categories of factors that influence the adoption of technology by SMEs. Although my research has not focused on what is traditionally defined as technology adoption, by analysing the interplay between elements of agency and structure I have shed some light onto the role of key participants during the introduction of new ICT. Additionally, the TiP analyses of Chapter 7 describe in further detail elements of the role of the key participants and how they encouraged and influenced the technology use of others.

The actions of key persons at AC (P2 \& P8) and CI (SMa \& SMb) emerged as an important influencing factor on peer consulting transition, as they went beyond simple managing or administering the transition process. I view their actions as exhibiting characteristics of leadership, or more precisely championing. The broadly accepted definition of championing is a person who acts as a supporter or a defender of a person, or more often of a cause, such as introducing online per consulting (Pinto \& Millet, 1999). I propose that there may exist a relationship between the championing concept and elements of the TiP framework (see Figure 15 of Chapter 7). 
As the analysis of Chapter 7 has demonstrated, participants' use of ICT was structured by existing facilities, norms and interpretive schemes (rules and resources). Such structuring enacted a specific set of rules and resources in their peer consulting practice that then served to structure future ICT use. Over an extended time period, members of $\mathrm{AC}$ and $\mathrm{CI}$ would constitute and reconstitute a particular structure of technology use, such as the Collaborative TiP and Isolated-Use TiP (respectively) described in this study. The "other structures enacted in the use of technology" (see the top right hand side of Figure 15 of Chapter 7) is of most interest here.

Orlikowski (2000) asserts that human interaction with technology will always enact other social structures along with the emergent TiP. Social structures are generally defined as sets of customs/norms, relationships, institutions, etc. which combine to form a social system (Giddens, 1994). Orlikowski gives examples of social structures that may be evident within a particular TiP as:

- authority structures

- cooperative culture

- incentive schemes

- legal requirements

This has particular significance when examining the link between the concept of championing and the TiP framework. I believe that it is reasonable to posit that the role or the type of role of the organisational champion can be included as one such social structure enacted through ICT use. It now falls to other researchers to examine the role of the champion, or change agent within the stages of the model and analyse in more depth the nature of their role and how their behaviours influence the technology use of others and overall progression through the stages of the model.

\subsubsection{The Distance Action Research approach}

A final academic contribution of this study is the use of the Action Research methodology. While I have followed the key tenets of Action Research, faced with the realities of the situations of the two participant organisations I have also extended 
the traditional action research method, and undertaken Distance Action Research (DAR) (see Chapter 3). The organisations that took part in this study are distributed across NZ, and have a small number of staff offering specialised services within diverse communities, different regions, and from large cities to remote and rural communities. It is this distributed organisational structure, along with other elements of the nature of $\mathrm{AC}$ and $\mathrm{CI}$ that gave rise to the organisational situation that I attempted to relieve through this research, and also demanded an adapted research methodology. As I was unable to meet with all participants face-to-face at frequent enough intervals to undertake key research activities, I successfully adopted and carried out DAR.

I now invite researchers who intend to study the introduction of new ICTs, processes or other change initiatives within distributed NZ organisations to use this adapted form of AR as a guide, and make further adaptations as necessary. For example, there would be value in extending the notion of DAR by examining the benefits and challenges of this method in more detail, and strategies to either take advantage of or overcome them. Also, researchers could now apply this method systematically and map out a DAR cycle that is distinct from the traditional AR cycle, offering IS researchers a valuable guide for those struggling to organise or operationalise their AR study.

\subsection{Implications for Practice}

Actively working with two participant organisations has provided some immediate benefit to the participant organisations and their staff, and the findings of this study now have potential to be of value to other NZ and international organisations. The following sections outline the implications of the research process and key findings to the academic and practitioner communities. Firstly, value was provided through highlighting and examining the primary research question: "how do NZ social services organisations apply online ICTs to enhance the professional development of their staff? " Examining this question was intended to provide value to organisations 
and their staff, as a greater understanding of this process would assist organisations in applying online technologies to span the distances over which they operate to provide more regular professional development activities for their staff. There are several aspects of the findings that resulted from pursuing this research question that have implications for practice.

The use of the Action Research method and extension to Distance Action Research (as discussed in Chapter 3) enabled the direct change intervention - introducing the online technology and peer consulting process - within each participant organisation. Compared with larger firms, the small to medium sized organisations in NZ usually cannot afford to employ staff with specialised technical expertise, or provide existing staff with the technology/systems training that is required for them to undertake such an intervention (Yoong \& Huff, 2000). Therefore, exposing key members of the participant organisations to new technology enabled them to gain a better understanding of the potential for ICT to add value within their organisation and industry. The use of Action Research method also enabled participants to gain first hand experience of these technologies, and of online peer consulting. This is an important aspect of this study, as the staff of many NZ human services organisations do not have a good understanding of, or experience with, the use of online ICT that support professional development and/or their work directly.

The MSD (2001, p.103) found that there was a "growing professionalism in counselling, advocacy, care and protection services" within the NZ environment. This shift towards increased professionalism presents NZ human services organisations with a challenge of providing improved professional development opportunities for staff. Participating in the peer consulting sessions as part of this study directly impacted on the professional practice of participants, for example at AC one participant stated:

"I have taken those ideas that came out of the supervision in regard to the case that I wanted to discuss and I used those with my client. And he's found, he's said that it was really good to have talked about the range of ways that we could deal through the process. So that has worked really well." (P2) 
The Model of Peer Consulting Transition provides organisations with some guidance to the stages that they are likely to encounter on the journey to introducing this type of non-traditional professional development activity, and the activities that are necessary within each stage. This knowledge will enable organisations to be better informed of the challenges that they may face and the behavioural, process and technology-related issues to consider. Additionally, the model descriptions of Chapters 5 and 6 highlight the important impact of the role and actions of key persons, or 'champions' in the development effort.

Linking to the stages of the model, the application of the TiP framework to elucidate the organisational factors at play within each organisation holds particular practitioner value. An understanding of the nature of particular organisational factors and how these form social structures (such as elements of organisational culture and structure), and their influence on peer consulting transition may assist organisations in determining their readiness for introducing online professional development. This knowledge could enable organisations to better prepare for the introduction of new forms of professional development activity and also for any changes in infrastructure sand/ or ICT required, leading to a greater possibility of successful outcomes.

The application of the TiP framework within the stages of the model holds another significant benefit for practitioners. Many frameworks that can be used to explore the interaction between people and technology begin with aspects or features/functions of the technology and then proceed to analyse how users engage with them. In contrast, the TiP framework has at its core the actions of people and how, through engaging with a particular technology a structure of technology use is enacted, called the technology in practice. By identifying the actions of staff as they interact with a technology during the course of their work, or professional development activities organisations can gain important insights into such issues as effective and ineffective behaviours, training requirements or incentive schemes. 


\subsection{Limitations of the Study}

The findings of this research have provided significant value to the participant organisations and participants groups within them. As described above, I believe that the outcomes of this study now offer value to practice and research in a number of ways. Although the benefits of the research are clear, issues of the validity of the research, the method adopted and of my researcher role, and of the organisational settings within which the research was conducted must be addressed.

The interpretive action researcher is not so concerned with the more traditional notions of internal and external validity. Instead, the concepts of plausibility, credibility, evidence and richness become key concerns (Hammersley, 1990). This research has adhered to the standards of:

- Plausibility: Within Chapters 5-7 I have provided convincing explanations of the actions that participants took when developing and participating in the online peer consulting programme. These chapters developed a storyline, based on the theoretical model developed (see Chapter 4) that was relevant and accepted by individual participants and the research organisations.

- Credibility: The narrative of this thesis was written in a way that is intended to immerse the reader in the contextual situation of each organisation and to show how the results reported emerged.

- Evidence: Evidence was collected mainly through interviews at all stages of the research. Throughout the thesis I have provided first-hand accounts of participants' experiences to illustrate and support key points and findings.

- Richness: I have presented detailed, situated descriptions of peer consulting transition at each organisation. Such descriptions have offered a research story of user actions in preparing for session and their engagement with online ICTs within peer consulting sessions, and the emergent technology and other social structures.

Although I have demonstrated the validity and relevance of this study, there are limitations that I will now discuss. Firstly, the DAR method offered limitations. As participants were situated across the country I conducted many interviews via phone and the online collaboration system. This was difficult at times due to the absence of contextual or non-verbal cues. Such cues provided additional richness to face-to-face interviews, which were conducted whenever possible. However, due to the nature of this research the DAR method enabled participants and I to use other 
communications channels such as email, cell phone, teleconference and meeting via the online collaboration system.

Another important limitation arises through the choice and application of ICTs in this study. As described in Chapters 5 and 6 (see, for example Section 5.2.2) and reiterated in section 8.3.2 above, the choice of Blackboard and Chatterbox involved an assessment of many factors affecting AC and CI. Such factors were systematically assessed through application of Media Synchronicity Theory (MST), which suggested that a combination of particular media choices would be appropriate for supporting peer consulting at $\mathrm{AC}$ and $\mathrm{CI}$, specifically:

- Face-to-face

- Telephone

- Tele/Video conference

- Asynchronous groupware (threaded discussions)

- Synchronous groupware (online chat, text and voice based)

While staff at both organisations already consulted via face-to-face, telephone (and/or cellular telephone) and tele/video conference mediums, the focus of ICT selection was on the provision of asynchronous and synchronous groupware. After consideration of the abovementioned factors, as well as the ease of use and technical set up and maintenance requirements, Blackboard and Chatterbox were chosen as the supporting ICTs for this study. While Chatterbox has been successfully used in nonacademic organisational environments for many years, Blackboard is primarily intended for educational support (as a course/learning management system) and its use in commercial settings has been limited to date. The use of Blackboard to support professional learning communities in this study is similar to application described in the case study of Watson Pharmaceuticals (Blackboard Incorporated, 2009), who created a continuous learning environment within which sales professionals engaged with their peers and managers before and after synchronous training events. Members of Watson Pharmaceuticals' sales force accessed resources such as information and tutorials through the online resource centre and also engaged regularly with peers and managers to discuss learning and training issues and events and receive feedback. 
While such use of Blackboard is increasing and provides a useful comparison to this study, the Blackboard software applied in this study was specifically designed for educational purposes, and could give rise to additional user resistance factors. For example, the University welcome and support mechanisms detailed on the Blackboard log in page may have confused participants, and the Menu structure was specifically designed for the higher tertiary academic environment. While I acknowledged and attempted to overcome such issues, their impact on ICT use at the Experimenting and Engaging stages of the study (as described in Chapter 5 and 6) remains largely uncertain.

My researcher role also raises some important issues that I wish to address. Prominent IS authors support and encourage various levels of involvement of the action researcher in the organisational setting, McKay \& Marshall (2001) arguing that "the active and deliberate self-involvement of the researcher in the context of their investigation" is one distinguishing feature of action research (p. 47). Within this research I adopted a role similar to that of the 'Expert' as described by Chaisson and Dexter (2001) and outlined in Chapter 3. Thus, I shared knowledge with participants and influenced their actions in certain appropriate ways at different stages of this study, while also allowing key organisational members to set the direction of the development of their online peer consulting programme. The value of my researcher role was described by many participants, P8 at AC stating: "having your input has just been fantastic. It's just really valuable that we've tried, and we've been supported to try it, I think that's been the key thing. I think there's probably lots of other organisations out there that'd benefit from that too, the 'have a go' sort of thing."

The research settings also included some limitations. Firstly, the findings of this research are derived from an in-depth study within two organisations. While this provides a high level of relevance, the generalisability of the study is reduced as I undertook a unique intervention within each of the two organisations and the results are specific to the experience of the participants, the study cannot be repeated exactly. However, the high validity of the study means that the findings can be generalised. In 
this case, I have generated the Model of Peer Consulting Transition, which can now be generalised to other organisational settings (as described above) and provide further practitioner and academic value.

Additionally, the regional offices of each participant organisation had varying levels of technical infrastructure that, at times, impacted participation in this study. For example, within CI some participants had dedicated access to a computer and a highspeed (broadband) internet connection. In contrast, the some participants in other regions had one shared office computer and slower, dial up internet access that could not be used while colleagues were on the phone or if a client was paying for services via EFTPOS. As the intent of this research was to study the process of applying ICT to support professional development activities within natural organisational settings, such limitations were reported and discussed within the chapters of this thesis and ultimately resulted in a richer narrative.

\subsection{Concluding Comments}

Through following the Distributed Action Research method and undertaking a change intervention within two participant organisations I have answered the primary research question of "how do NZ social services organisations apply online ICTs to enhance the professional development of their staff?" The Model of Peer

Consulting Transition that I generated through this study reflects the five stages and various activities that participants moved through when transitioning from traditional peer consulting activities to an online programme of peer consultation. I extended this mapping, and applied the TiP framework to explore participants' interaction with ICT within online peer consulting sessions. I found a number of key organisational factors that impacted their technology use and progress through the stages of the model.

The research process followed and the findings reported here provide benefits to practitioner and academic communities. I am now interested to see how practitioners 
and researchers will apply and extend the Model of Peer Consulting Transition and application of the Technology-in-Practice framework. 


\section{Appendix A - Community of Practice Frameworks}

This Appendix discusses various community of practice models developed by prominent authors in the field. Firstly, Wenger's cross-sectional view of CoPs including Domain, Community, and Practice elements is discussed as a key example. Next, a set of three models that look at community development stages are discussed.

Wenger, McDermott \& Snyder (2002) posit that communities of practice share a basic structure that is combined of three fundamental elements: domain, community and practice (p. 27).

\section{Domain}

The domain is where common grounds and a sense of common identity are created. Generally, a domain consists of key issues/problems that members commonly experience. As such, it is not a fixed set of problems but evolves along with the external environment and the community. A domain inspires members to contribute and participate, guides their learning and gives meaning to their actions, allowing them to see what is worth sharing among community members. Within a community a shared domain creates a sense of accountability to a body of knowledge and development of a practice.

\section{Community}

The community creates the 'social fabric' of learning within the group by fostering interactions and relationships based on mutual respect and trust. A CoP is essentially a group of people who interact, learn together, and build relationships. These interpersonal relationships are critical, as knowing each other makes it easier for members to share ideas and ask questions. Over time, interactions become more regular and on issues important to the domain. As interactions take place, a community builds relationships based on trust and a sense of common history and identity.

\section{Practice}

This element encompasses a set of frameworks, ideas, tools, information, language, stories, documents and repositories that $\mathrm{CoP}$ members share. Essentially, the practice of a CoP consists of a set of socially defined ways of doing things in a specific domain: a set of common approaches and shared standards that create a basis for action, communication, and problem solving within the group. The practice can also be described as the specific knowledge the community develops, shares and maintains that explores both the existing body of knowledge related to the domain and the latest advances in the field.

Each community has a certain way of making its practice visible through the ways that it develops and shares knowledge, examples include stories, formulas, 
procedures in articles, best practices and discussions. Successful practice development depends on a balance of joint activities and the production of knowledge items such as documents and tools.

Wenger et al. (2002) state that when Domain, Community and Practice function well together, a CoP can evolve into an ideal 'knowledge structure' - a social structure that can assume responsibility for developing and sharing knowledge (p. 40). Defining domain, community and practice helps to clarify the definition of a $\mathrm{CoP}$ as a social structure and distinguish it from other social structure types. In addition, the model provides a 'common language' for a community that facilitates discussion, collective action and efforts to gain legitimacy, funding and sponsorship in an organisation.

A discussion of three different models of community of practice development, created by Wenger (1998), McDermott (2000) and Gongla \& Rizzuto (2001) is below.

\section{The Stages of Development model (Wenger, 1998)}

Wenger (1998) postulated that, over time CoPs move through various stages of development that are characterised by levels of interaction among members and the different kinds of activities that they undertake. Wenger (1998) developed a 'Stages of Development' model comprised of five stages: Potential, Coalescing, Active, Dispersed and Memorable (see Figure 18).

\section{Stages of Development}

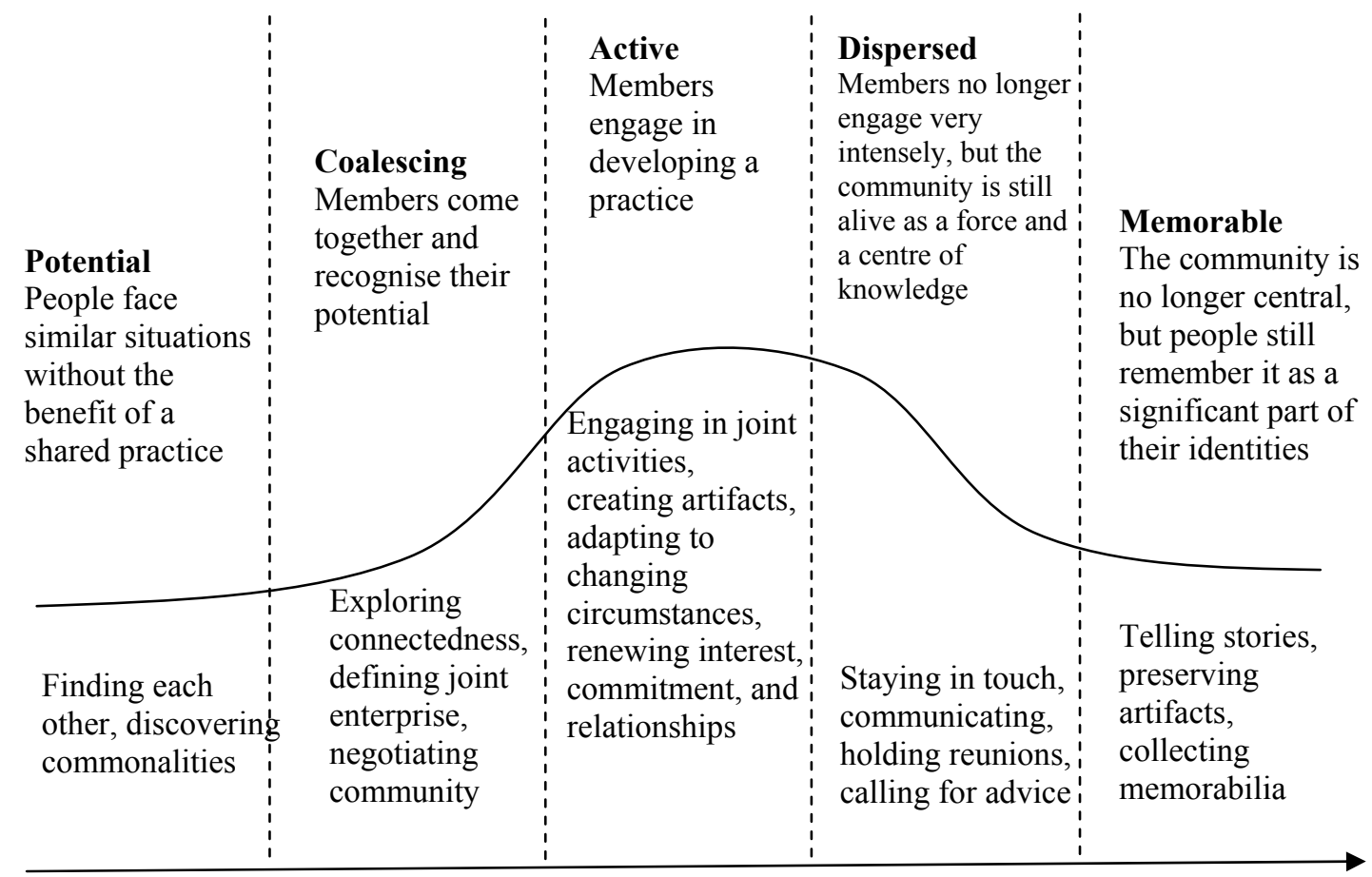

Figure 18: Stages of Development (Wenger, 1998; p. 3). 
At the Potential stage, people in an organisation are facing similar problem situations, without the benefit of a shared practice. There is the potential for a community to form to tackle such problems and jointly generate possible solutions. Activities at this stage include potential community members locating each other and engaging in discourse to discover commonalities.

At the Coalescing stage people come together and recognise the potential value that a community could bring to them as a group, as individuals and the wider organisation. At this stage the group explores possible connectedness, defines joint enterprise and negotiates a 'community' definition.

At the Active stage community members engage in developing a particular practice. Many activities are undertaken: engaging in joint activities, creating communal artefacts, adapting to changing circumstances, renewing interest in the community and practice area, and building commitment and relationships among community members.

The Dispersed stage is where members no longer engage intensely, but the community is still alive and recognised as a 'force' within the organisation and as a centre of knowledge. Activities here are: staying in touch and communicating within the community, holding reunions and calling community members for advice.

During the final stage, Memorable, the community is no longer recognised as central, but still remembered or referred to as a significant part of their identities. Activities at this stage are centred on telling community stories, preserving artefacts and collecting memorabilia.

These stages build on each other, over a period of time, and a CoP needs to fulfil the goals/ reach the outcomes of one stage before moving on to the next. As such it is not possible for a community of practice to move from the active stage back to the coalescing stage, for example. Therefore, over time a community is born at the Potential stage, matures through the Coalescing and Active stages, begins to die off at the Dispersed stage and finally ceases to exist at the Memorable stage.

\section{The Stages of Community Development model (McDermott, 2000)}

McDermott (2000) developed The Stages of Community Development model that is based on a life-cycle approach similar to that of Wenger (1998), discussed above. Each stage of community development, like each stage of human development, poses tensions that a community needs to resolve before it can move to the next development stage. A community must experience such tensions, wrestle with them and come to a resolution that propels them to the next development stage. 
As with the previous model, McDermott's community development model incorporates five stages: Plan, Start Up, Grow, Sustain/Renew and Close (see Figure 19).

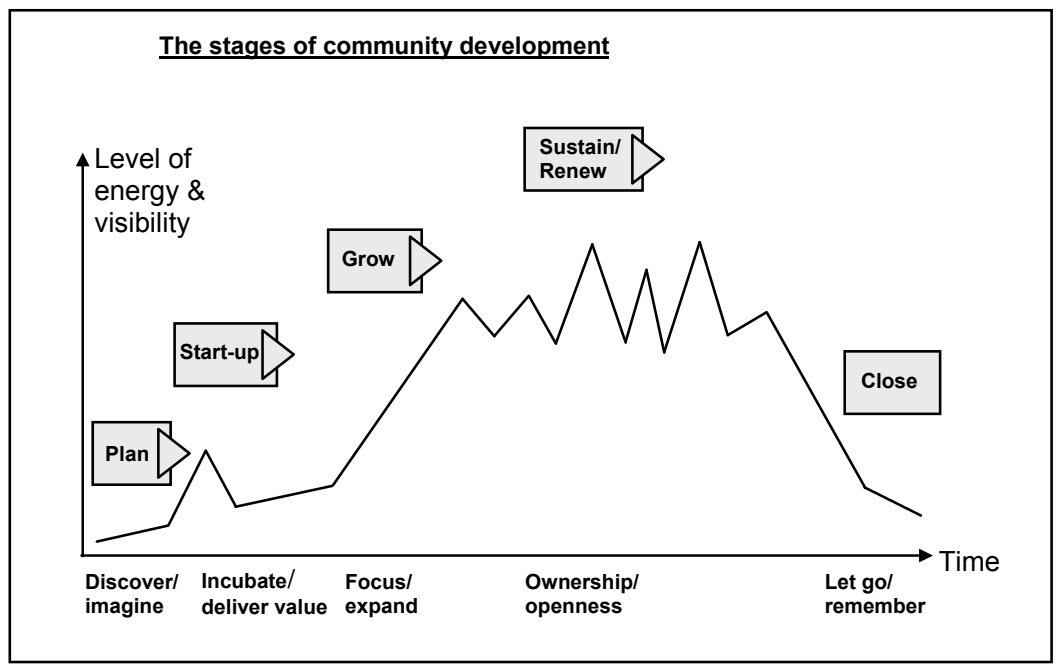

Figure 19: The Stages of Community Development (McDermott, 2000; p. 17).

At the Planning Stage, a community emerges out of members' existing social networks. There needs to be careful thought and planning to expand the community from a group of people that share an interest to a community that is concerned with developing a practice.

The Start Up stage is where a 'spike' of interest and/ or excitement encourages the community to form, although as individuals' time and energy are consumed this excitement may decrease. The community faces a tension between 'incubate or deliver': do they develop relationships and decide what is valuable to share or attempt to deliver immediate value to those involved? One is usually at the cost of the other, and the community will need to assess which is more urgent for their survival.

During the third stage of development, Growth, the community of practice responds to interest from newcomers and may expand their membership from the core group. The decision faced here is whether to focus or expand. Growth may bring the community new ideas and perspectives, but it will expose the core members to outsiders. Tension may be created if newcomers disrupt the pattern of interaction that the core community had developed, and this disruption of norms can lessen the sense of intimacy and familiarity felt by the core group.

Sustain is where an active community goes through periods of high and low energy as it responds to changes in its practice, membership and relationship to the organisation. The community is then faced with deciding how to sustain its momentum through these changes. They need to develop a strong sense of ownership of their shared knowledge to maintain a strong sense of practice. In order to prevent 
stagnancy, the community should remain open to new ideas, new membership and changes in discussion topics. Sustaining momentum is important as it can help community leaders see opportunities for the community to take on new challenges such as accepting new members, and new opportunities to apply their insights.

Close is the final stage in this model where the community dies. This can occur in two ways: 1) The community may fade away, losing members until finally no one turns up any more, or 2) the community can turn into a 'social club' as it loses its sense of guiding a practice. Community members decide whether to let the community fade away or to 'articulating its legacy' by preserving documents and identifying key community insights and individuals within the organisation who can carry them on.

These stages take place in relation to time, and a $\mathrm{CoP}$ must experience, struggle with and resolve various tensions at each stage before moving on to the next. Like Wenger's model, it is only possible to move through the community development model sequentially from the Plan stage through to the Close stage.

\section{The Community Evolution Model (Gongla \& Rizzuto, 2001)}

Gongla and Rizzuto spent about five years studying over sixty communities of practice at IBM. They presented a model for CoP development based on an 'evolution pattern' than can be summarised into five stages: potential, building, engaged, active and adaptive (see Table 29).

\begin{tabular}{|c|c|c|c|c|c|}
\hline & Potential & Building & Engaged & Active & Adaptive \\
\hline Definition & $\begin{array}{l}\text { A } \\
\text { community } \\
\text { is forming }\end{array}$ & $\begin{array}{l}\text { The } \\
\text { community } \\
\text { defines } \\
\text { itself and } \\
\text { formalises } \\
\text { its } \\
\text { operating } \\
\text { principles }\end{array}$ & $\begin{array}{l}\text { The } \\
\text { community } \\
\text { executes } \\
\text { and } \\
\text { improves } \\
\text { its } \\
\text { processes }\end{array}$ & $\begin{array}{l}\text { The } \\
\text { community } \\
\text { understands } \\
\text { and } \\
\text { demonstrates } \\
\text { benefits } \\
\text { from } \\
\text { knowledge } \\
\text { management } \\
\text { and the } \\
\text { collective } \\
\text { work of the } \\
\text { community }\end{array}$ & $\begin{array}{l}\text { The } \\
\text { community } \\
\text { and its } \\
\text { supporting } \\
\text { organisation(s) } \\
\text { are using } \\
\text { knowledge for } \\
\text { competitive } \\
\text { advantage }\end{array}$ \\
\hline
\end{tabular}

Table 29: Community Evolution Model Definition (Gongla \& Rizzuto, 2001; p. 845).

This evolution model describes those characteristics that distinguish communities in one stage from those in another. The model illustrates that communities are distinct at each stage, having different characteristics as they move from stage to stage. It is 
important to note that the model supposes that at each of the later stages, the functions and characteristics of the previous stages already exist to some degree.

As evident from the discussions above, the models of Wenger (1998) and McDermott (2000), both adopt 'life-cycle' approaches to community development whereby the stages of development are not unlike the stages of birth, maturation and death. Gongla \& Rizzuto's model clearly has similar elements to both the Wenger and McDermott models, as it recognises formative and growth stages of community of practice development. However, the evolution model proposed by Gongla \& Rizzuto (2001) is not a lifecycle approach. Within their evolution model, a community can mature and dissolve at any one of the four stages beyond the initial formation level: "the model describes instead how communities transform themselves, becoming more capable at each stage, while at the same time maintaining a distinct, coherent identity throughout" (Gongla \& Rizzuto, 2001; p. 846).

Each stage of the evolution model has fundamental, or underlying functions. These functions help to explain why one stage is different from the next and show what a community needs to build on from previous stages (see Table 30).

\begin{tabular}{|l|l|l|l|l|l|}
\hline & $\begin{array}{l}\text { Potential } \\
\text { Stage }\end{array}$ & Building Stage & $\begin{array}{c}\text { Engaged } \\
\text { Stage }\end{array}$ & \multicolumn{1}{|c|}{$\begin{array}{c}\text { Active } \\
\text { Stage }\end{array}$} & $\begin{array}{c}\text { Adaptive } \\
\text { Stage }\end{array}$ \\
\hline $\begin{array}{l}\text { Fundamental } \\
\text { Functions }\end{array}$ & Connection & $\begin{array}{l}\text { Memory and } \\
\text { context creation }\end{array}$ & $\begin{array}{l}\text { Access and } \\
\text { learning }\end{array}$ & Collaboration & $\begin{array}{l}\text { Innovation } \\
\text { and } \\
\text { generation }\end{array}$ \\
\hline
\end{tabular}

Table 30: Fundamental Functions for the Stages of Evolution (Gongla \& Rizzuto, 2001; p. 846).

By grouping their observations into a 'capability oriented' model, Gongla \& Rizzuto were able to describe the variations in behaviour or people, process and enabling technology as communities evolved:

- People: social individuals and their individual and group behaviours, as well as the larger organisational behaviour influence

- Processes: "sets of documented steps with clearly defined roles and activities for people to perform" (p. 848). This includes internal (community-oriented) and external (organisational) processes

- Technology: application of science and the body of IS knowledge that can be used to fashion tools, practice knowledge arts, and extract the needed data and information

These constructs are essentially 'influencing elements' on the evolution process. For example, they are the enablers for promoting a connection at the Potential stage (see Table 31). 


\begin{tabular}{|l|l|}
\hline STAGE & POTENTIAL \\
\hline Fundamental Function & Connection \\
\hline People Behaviour & Individuals find one another and link up \\
\hline & $\begin{array}{l}\text { The organisation may be unaware of or uninterested in the } \\
\text { potential community OR the organisation may provide some } \\
\text { support to locate and introduce individuals }\end{array}$ \\
\hline Process Support & Identifying potential community members \\
\hline & Locating potential community members \\
\hline Enabling Technology & Facilitating bringing community members together \\
\hline & Electronic messaging systems: email, chat rooms, lists \\
\hline & Phone calls and teleconferences \\
\hline & On-line forms \\
\hline & On-line directories \\
\hline
\end{tabular}

Table 31: Potential Stage Enablers that Promote Connection (Gongla \& Rizzuto, 2001; p. 846).

Specific to IS research, the community evolution model developed by Gongla \& Rizzuto (2001) holds some important advantages over the Wenger (1998) and McDermott (2000) models. Most apparent is the inclusion of enabling technology factors and their influence on promoting the fundamental functions of each stage. This provides a direct link between community of practice development and information technology and systems that is absent from other community development models. The evolution model also makes it possible to describe how the various people, process and technology factors work together to nurture and sustain a community and enable to evolution from one development stage to the next.

This model does not assume that CoPs can only contribute value to the organisation at the final or later stages of evolution. As a CoP begins to actively steward a practice and develop knowledge items relevant to that practice, the wider organisation may experience value in terms of increased productivity, the development of new work procedures and practices, and increased innovation. This is important, as the formative, development stages of the evolution model are not strictly sequential, as in Wenger's (1998) and McDermott's (2000) model. Within the evolution model CoPs may stay at certain stages and not evolve to another level of the model, move backward and forward between the stages, have some characteristics of one stage while still primarily at another stage, or they may 'rest' for extended periods at one stage and suddenly evolve quickly to another stage (Gongla \& Rizzuto, 2001). The ability for a community to build capacity and add value to the organisation at any stage of the evolutionary process is therefore advantageous. 


\section{Appendix B - Codes List}

This Appendix provides an example of a key list of codes used to analyse data and generate themes and categories that led to the Model of Peer Consulting Transition.

\begin{tabular}{|l|l|l|}
\hline Code & Communities of Practice & \\
\hline CoP_FTF & Evidence of community & Evidence of face-to-face community \\
\hline CoP_OL & & Evidence of online community \\
\hline CoP_Grw & & Growth of community \\
\hline CoP_Pos & Positive aspect of community & \\
\hline CoP_Neg & Negative aspect of community & \\
\hline CoP_Potential & Potential Stage factors & Forming, connection (People, process or tech factors) \\
\hline CoP_Building & Building Stage factors & Defining, formalising operating principles, memory creation \\
\hline CoP_Engaged & Engaged Stage factors & Executes and improves processes, access and learning \\
\hline CoP_Active & Active Stage factors & Benefits from KM, collaboration \\
\hline CoP_Adapt & Adaptive Stage factors & Using KN for competitive advantage, innovation and generation \\
\hline Code & Technology & \\
\hline Tech_ExpComf & Experience/comfort with IT & \\
\hline Tech_Bb & Type of technology mentioned & Blackboard \\
\hline Tech_Cbx & & Chatterbox \\
\hline Tech_Email & & Email \\
\hline Tech_Phone & & Phone \\
\hline Tech_Other & & Other (notes, paper-based, fax) \\
\hline Tech_Access & Access to technology & Any issues with accessing computers/Internet \\
\hline Code & Blackboard & \\
\hline Bb_Train & & Training given/needed \\
\hline Bb_Feat & & Features need \\
\hline
\end{tabular}




\begin{tabular}{|c|c|c|}
\hline $\mathrm{Bb}$ Use & & Ease of use \\
\hline Bb_Format & & Format (text based) \\
\hline Bb_Cues & & Absence or presence of cues (social, verbal, non-verbal) \\
\hline Bb_Diffic & & Difficulties with Blackboard use (technical) \\
\hline Bb_Value & & Value derived from engagement via Blackboard \\
\hline Code & Chatterbox & \\
\hline Cbx_Train & & Training given/needed \\
\hline Cbx_Feat & & Features need \\
\hline Cbx_Use & & Ease of use \\
\hline Cbx_Format & & Format (voice based) \\
\hline Cbx_Cues & & Absence or presence of cues (social, verbal, non-verbal...) \\
\hline Cbx_Diffic & & Difficulties with Chatterbox use \\
\hline Cbx_Value & & Value derived from engagement via Chatterbox \\
\hline Code & Relationships & \\
\hline Relat_Prob & Problems within relationships & Any influence felt or suggested \\
\hline Relat_Isolatn & References to isolation & Isolation - professional and/or social and/or geographical \\
\hline Relat_Comm_Diff & Communications difficulties & Communication problems (attitudinal also) \\
\hline Relat_Comm_Suprt & Pre-existing relationships & Influence on communications format - Supportive/encouraging \\
\hline Relat_Comm_Hindr & & Influence on communications format - Hindering/inhibiting \\
\hline Relat_OL_Dev & Online Relationships & Relationship development \\
\hline Relat_OL_Maint & & Relationship maintenance \\
\hline Relat_OL_F\I & & Formal \Informal \\
\hline Code & Champion Role & \\
\hline Ch_Who & Evidence of & Who is acting as a champion? \\
\hline Ch_Facilit & Characteristic of championing & Facilitation / Coordination, Driver, Cheerleader etc \\
\hline Ch_Passn & & Passion / enthusiasm / encouragement / motivation \\
\hline Ch_Lead & & Leadership (traditional, evidence of...) \\
\hline Ch_Method & Championing at project stages & Methodology stage - developing approaches, concepts etc \\
\hline Ch_Actn & & Project 'action' stage - changing processes, sessions etc \\
\hline
\end{tabular}




\begin{tabular}{|c|c|c|}
\hline $\mathrm{Ch} \_\mathrm{CoP}$ & Championing at CoP stages & Which stage? (or Model stage) \\
\hline Code & Researcher Role & \\
\hline Role_ARD_Method & Any references to ARD stages & \\
\hline Role_ARD_Media & AR at a distance & Media/Channels used \\
\hline Role_ARD_Int & Distance Interviewing & \\
\hline Role_ARD_Contact & & Difficulties with AR at a distance \\
\hline Role_RrVis_Hi & Visibility at AR stage & High visibility of researcher \\
\hline Role_ProjVis_Hi & & High visibility of project \\
\hline Role_RrVis_Lo & & Low visibility of researcher \\
\hline Role ProjVis Lo & & Low visibility of project \\
\hline Role_Com_Media & Communication & Role / use of media \\
\hline Role_Com_Freq & & Frequency of communication \\
\hline Role_Com_Prob & & Communications difficulties \\
\hline Role_Com_Tone & & Formality / tone of communication \\
\hline Role_Collab & Collaboration & Evidence of working collaboratively with key participants \\
\hline Role_ChAgnt & Change agent & How have I acted to bring about change? \\
\hline Role_Train_Bb & Trainer & Blackboard (including sessions, resources and ideas) \\
\hline Role_Train_Cbx & & Chatterbox (including sessions, resources and ideas) \\
\hline Role_TechSup & Technology support & Both initial and ongoing support provided \\
\hline Role_Relat_Ch & Relationships with participants & Champions/leaders \\
\hline Role_Relat_Other & & All other participants \\
\hline Code & Organisational Culture & \\
\hline Cul_Pos & Aspects of culture & Positive impact on project and/or sessions \\
\hline Cul_Neg & & Negative impact on project and/or sessions \\
\hline Cul_Reinf & Actions that reinforce culture & Strength of organisational culture \\
\hline Cul_Change & Actions contrary to culture & Changing culture \\
\hline Code & Peer Consulting Process & \\
\hline Proc_Struct & Structuring of the process & \\
\hline Proc_Plan & Pre-session planning actions & \\
\hline
\end{tabular}




\begin{tabular}{|l|l|l|}
\hline Proc_Plan_Media & Tech used in session planning & Email/phone needed in the setting up of sessions \\
\hline Proc_Steps & Steps of an actual session & \\
\hline Proc_Duratn & Duration of a session & \\
\hline Proc_Value & Benefit/ value of a session & An OL session \\
\hline Proc_FTF_Sim & FTF \& OL session & Similarities between \\
\hline Proc_FTF_Diff & FTF \& OL session & Differences between \\
\hline Proc_Inhbt_Tech & Inhibits participation & Technology related factors \\
\hline Proc_Inhbt_Othr & Inhibits participation & Other factors (not tech related) \\
\hline Proc_Encrg & Encourages participation & Factors that encourage participation \\
\hline
\end{tabular}

Table 32: Example Codes List 


\section{Appendix C - AC Scoping Document}

This Appendix presents the scoping document developed at AC's Scoping stage.

Proposal for joint research project between $\mathrm{AC}$ and the School of Information Management

Victoria University of Wellington

by

Julie Abbott

PhD candidate

School of Information Management

Victoria University of Wellington 


\section{Introduction}

The sharing of professional knowledge contributes to the success of all organisations. When organisational members are based at different geographical locations the sharing of knowledge often comes at a significant cost and inconvenience. However, practice-related problems, process innovations and program developments must still be shared between professionals in order for an organisation to progress and evolve.

As there is typically a vast amount of new knowledge generated during the start-up phase of an organisation, the processes of collecting, organising and sharing this new knowledge can be critical. However, without adequate infrastructure geographically dispersed staff members can soon miss out on participating in these processes, and sharing in and applying the new knowledge that is generated at another location.

\section{Purpose}

The purpose of this research project is to explore how information communication technologies can support the staff of geographically distributed organisations in collecting, organising and sharing professional knowledge.

Currently within AC there are few opportunities for staff from around the South Island to meet face-to-face and share knowledge in peer consulting sessions. As all staff face limitations in terms of their available time and finances, they cannot always gain the benefits of consulting with others. This may result in some staff being unaware of recent process and program innovations and a delay in the opportunity to further develop such processes and programs and apply innovations.

In addition to this, counsellors are faced with difficulties surrounding the accessibility and availability of documentation, as 'parcels' of information exist within each staff member's particular location.

\section{Project scope}

In response to the situation outlined above, Julie proposes the adoption of a simple online system that will enable AC professionals to discuss the practice-related problems and issues that are important to them and exchange the necessary documentation that will contribute to their professional development. It is anticipated that the overall management of $\mathrm{AC}$ will also be positively affected.

With the support of technology, groups of practitioners could engage regularly over the Internet to share professional knowledge, reducing the need to travel for face-toface meetings. More regular connections could be made through this system between geographically separated practitioners, supporting the communications and 
collaborations that are needed for the collection, organisation and sharing of knowledge to take place.

An online system could also support the development of documentation (eg policies), the sharing of documentation, and act as a central repository for selected documentation around a process or program. In this way, the most current versions of important documents would be available in one central store and accessible to all staff at any time.

An effective way of experimenting with a simple online system may be to focus on a discrete component of AC's work, e.g. the Journey component of the ADC programme. A journey requires a great deal of communication and collaboration. This would likely be enhanced by the system described above. Including:

- The pre journey preparations including logistical tasks as well as client and programme risk management

- The on journey phase as captured within journey reports, briefing of counsellors not present with clients on the journey, debriefing critical incidents etc.

- And post journey tasks such as procedure and document updating, etc.

A single journey includes many counsellors, and the collaborations that take place each day create a unique opportunity for peers to learn from each other and generate new knowledge. The insights and new knowledge generated need to be shared with other AC counsellors who participate in the journey program.

- How can this new knowledge be captured?

- How can this new knowledge be discussed and shared it in a timely way?

- How can the innovations from one journey feed into the preparation for the next journey, no matter how soon it is?

Here, a simple online system could prove most effective. During the journey notes are taken and discussed by the group of counsellors. At the conclusion of the journey these notes are then collated into a journey report that is shared among the attending counsellors. There are two main ways that an online system could assist here. Firstly, the evolving report could be posted and a text based discussion board used to pose questions and seek responses from peers or to seek more general feedback. An online real-time discussion ('chat') could then take place based around the key feedback points offered. Any learning that takes place and results in new organisational knowledge could then be documented and posted in the system somewhere for others to see. These could also be written into another version of the journey report, which could be stored in an appropriate online repository that is accessible by AC staff at any time, from anywhere. 
The outcomes of a particular journey could be used to influence the preparation of the next journey - counsellors responsible for the next journey could simply go to the online repository and view the relevant journey report(s) and look back over the discussion to note any additional explanations of points or issues that may be important for them to know. This would also be an opportunity for the program developer and/or management team to view both the journey documents and the discussions to be aware of program innovations and potential program developments and offer their suggestions.

It is anticipated that this scenario could be replicated to support the interactions that are needed within many organisational processes, such as forming policy documents, developing new program areas, or preparing a tender for a contract.

An online system would be intended not as a replacement for the current face-to-face meetings and peer consulting methods that exist within $\mathrm{AC}$, but rather as an extension of them, adding another dimension to work and learning.

\section{Significance of the project}

The main benefit of this project will be the development of an alternative way to enable AC staff to share professional knowledge on a more regular basis, no matter where in the South Island they are. The organisation may not only save time and financial resources, but the staff will benefit directly and immediately through being part of a much closer community of practitioners.

Within this technology-supported community they will be able to share and discuss the most up to date organisational issues, information and documentation. Such information can then be easily distributed to others within AC. Through engaging with peers on a more regular basis the latest program innovations can be shared soon after they happen and opportunities to further develop the programs and directly benefit AC clients will not be lost.

It is anticipated that the management of $\mathrm{AC}$ as an organisation will also be positively affected through the management team having increased opportunities to share professional knowledge and increased access to organisational documents. In addition, having a more direct link to the experiences of AC counsellors will offer greater insight into the running of programs. It is also anticipated that the learning that ensues from participating in this joint research project will be directly transferable to other process and programs within AC.

\section{Action Research}

The action research method will be used in undertaking this research project. Action research is cyclical in nature and involves the following steps: 
- assessing the current organisational situation

- putting in place the online system to help relieve the identified problem

- interviewing AC staff to gather their insights and experiences of using the system

- conducting data analysis to assess the outcomes of the actions taken

- presenting the outcomes to $\mathrm{AC}$ in the form of a report

The introduction of a pilot online system for peer consultation is the centre of the 'action' component of the study. Julie will mainly work from a distance in collaboration with various members of AC. Assessing the current organisational situation, setting up the pilot system and testing its usefulness in real work situations will mainly involve interviews with staff during the life of the project to gain their perspectives and experiences on this topic. Most interviews would be conducted via phone, although some fact-to-face interviews may be necessary. It is expected that the joint research project between AC and Julie Abbott would be over a period of time not greater than one year.

Within any research project issues of privacy and security arise. Formal documents outlining the privacy and security of research material will be presented AC staff before any data is collected. The privacy of data that is collected during this project is guaranteed. Julie Abbott will ask for written informed consent before collecting any data, whether through interviews or other means (such as documentation). All data collected will be kept in password-protected files on Julie's personal computer. She will be the only person who has access to such files, although Associate Professor Pak Yoong ${ }^{20}$ may need to view particular data from time to time. Written/ hard copy data, reports etc will be kept under lock and key, and again Julie will be the only person with access to such material.

When reporting the findings of this research, $\mathrm{AC}$ and individuals within $\mathrm{AC}$ will not be identified in any way, unless express written permission from the organisation and individuals concerned is obtained. At the conclusion of the research project $\mathrm{AC}$ will have the option of having data returned, or Julie will destroy all data collected.

When using online information systems the issues of privacy and security are important. Each separate system used has in-built security features, namely they are username and password protected. Each research participant will be given a unique username and password for the systems involved. Specific users will be given access to the actual sites that will be used for this pilot project, meaning that access is restricted to particular AC staff and Julie Abbott and Associate Professor Pak Yoong. There are also other technical administration personnel of Victoria University that may access the sites from time to time, but Julie will be aware of this and can advise you accordingly. No one will have access to the sites that will be used in this study

\footnotetext{
${ }^{20}$ Assoc. Prof. Pak Yoong is Julie's PhD supervisor.
} 
without legitimate users being aware of their presence - ie no one can 'lurk' anonymously in the background without your knowledge.

There is an additional issue of the privacy and security of information about the clients of AC. At this stage it is not expected that Julie would need to collect or view any information relating to any particular clients, whether 'online' or in a physical document. If, during the course of this project AC staff do need to mention particular clients, the use of pseudonyms or other identifiers (such as client numbers) could be used in replacement of actual names, protecting the identity of clients and the privacy of client information.

\section{Summary}

Professionals within geographically dispersed organisations, especially start-ups, may experience difficulties when attempting to engage in knowledge sharing. The processes of capturing, organising and sharing knowledge cannot be ignored and are critical to the development of organisational processes and programs and the organisation as a whole.

Through this research project $\mathrm{AC}$ will have the opportunity to assess and evaluate the usefulness of a simple online system in supporting organisation-wide knowledge sharing. The benefits of participating in this project are many, including the creation of a closer community of professionals, more regular sharing of knowledge about processes and counselling programs, as well as the time and financial resources that may be saved. The learning gained from this project will most likely be transferable to other organisational situations in the future.

Julie Abbott is a PhD student within the School of Information Management at Victoria University, Wellington. Her research interest is in the various ways that technology can support people that are working at different geographical locations. Assoc. Prof. Pak Yoong supervises Julie.

Julie Abbott

Email: Julie.Abbott@vuw.ac.nz Phone: 0210714651
Assoc. Prof. Pak Yoong

Email: Pak.Yoong@vuw.ac.nz

Phone: 044635878 


\section{Appendix D - Media Synchronicity Theory}

This appendix includes additional detail on Media Synchronicity Theory (MST). This theory was used to advise the researcher and research participants on the ICT features that could most appropriately support online peer consulting.

\section{Selection of MST}

Media richness theory, also called information richness theory was developed by Shannon \& Weaver (1949) and applied and extended by such researchers as Weick $(1979 \ldots)$ and later extensively by Daft \& Lengel (1986...). This theory is concerned with task performance and essentially argues that this performance will be improved when task needs are matched to a mediums ability to convey information. While addressing the processing of information, this argument focuses on the degree of social presence that a medium is able to convey. The theory assumes a hierarchy of media and ranks a set of media (such as face-to-face, email, phone etc) from 'richest' to 'leanest'. Richness of a medium is related to its ability to convey information and change understanding within a time interval.

Media richness theory assesses the richness of media when groups undertake the tasks of reducing equivocality or uncertainty (Daft \& Lengel, 1986; Lengel \& Daft, 1988). Tasks that are highly equivocal centre on a lack of understanding and those tasks that are highly uncertain generally involve a lack of information (Weick, 1979). Media richness theory argues that highly equivocal tasks require media with high richness, such as within the face-to-face environment and that a highly uncertain task will have higher performance when lean media are used, such as written mail/ electronic mail (Lengel \& Daft, 1988).

There is a well-established stream of research in the psychology, organisational behaviour and information systems disciplines that examines the matching of media to a task to improve group and organisational performance. However, with changing internal and external organisational environments and the constant introduction of new technological innovations into organisational life, some researchers have questioned the appropriateness of media richness theory to the modern organisational situations and have argued for the application of theories alternative to media or information richness theory.

Such researchers have turned to other theories to help explain the communications events and media choices present within the organisational situations that they have studied, for example Task-Media Fit Hypothesis (as discussed by Mennecke, Valacich \& Wheeler, 2000) Genre Theory (see for example Yates \& Orlikowski, 2002) and MST (see for example Carlson \& George, 2004 and Maruping \& Agarwal, 2004). 
Following the reasoning of these researchers, media richness theory was found to go only part way to describing the media that could best support the communications within the peer consulting process at CI. Thus, MST was selected and applied, as next discussed.

\section{The nature of MST}

Synchronous activity is defined as that which occurs or moves at the same rate and exactly together (www.oed.com). Media synchronicity refers to the extent to which individuals within a larger group are able to work on the same activity at the same time, also termed a 'shared focus' (Dennis \& Valacich, 1999).

Dennis \& Valacich (1999) developed MST based on the argument that the key to effective media use is the matching of media capabilities to the primary

communication processes required to perform a particular group task. MST is said to extend the ideas of media richness theory by still includes communications at the task level, but also devolving to a further level of abstraction to assess media that can best support communications processes that are inherently within tasks.

These communication processes are termed conveyance and convergence and when combined with five key media capabilities communications effectiveness can be improved.

\section{Conveyance}

Conveyance is the dissemination of a diversity of information from many sources, information previously unknown to participants. The goal of conveyance is to disseminate and obtain as much relevant information as possible to aid in understanding the situation at hand. Once information is shared through conveyance, a second fundamental process is necessary: Convergence.

\section{Convergence}

This refers to the convergence on a shared meaning of the information. The focus here is on understanding each individual interpretation of the information, not the information itself. The goal of convergence is for a group to agree on the meaning of this information in the context of the current situation. Those involved need to reach a common understanding and agree that they have achieved this understanding (or that common understanding is not possible). Convergence uses a comparison process whereby individuals compare their conclusions to those of others in a group.

MST posits that matching these communications process to certain media characteristics can improve communications performance (see Table 33). Media richness theory developed a set of media characteristics: language variety, multiplicity of cues, personalization and rapid feedback. MST extends this set, 
combining language variety and multiplicity of cues into the characteristic of symbol variety, renaming rapid feedback immediacy of feedback, and distributing elements of personalization into parallelism, reprocessability and rehearsability, as defined:

- Immediacy offeedback

This characteristic is concerned with the extent to which a user can give rapid feedback on a communication that they receive. With regards to a particular medium, it is the ability of that medium to support "bi-directional communication".

- Symbol variety

Symbol variety is the number of ways that information can be communicated. Dennis \& Valacich (1999) described four ways that symbol variety can affect communication and understanding of messages:

1. Some information may be easier to convey in one format as opposed to another

2. Verbal and nonverbal symbols enable the sender to include information beyond the words themselves when a message is transmitted

3. The cost to compose a message or to process an incoming message using some symbol set may impose a delay cost or production cost that alters the way in which the sender creates message or reduces the understanding of the receiver

4. Lack of verbal and nonverbal symbols can have significant effects on social perceptions. ie, when verbal/nonverbal cues are removed there is a loss of social presence, such that the people with whom one is communicating become less like real people and more like objects

- $\quad$ Parallelism

This refers to the number of simultaneous conversations that can exist effectively, also termed "multiple addressability". For example, with the use of the telephone, only one conversation can effectively use the medium at one time. Contrastingly, many electronic media can be structured to enable many simultaneous conversations to occur. However, it is important to note that as the number of conversations increase, it becomes more difficult to monitor and coordinate the conversations.

\section{- Rehearsability}

This characteristic refers to the extent to which media enables the sender to rehearse or fine tune the message before sending, also termed "editability". Some media allow the sender to carefully edit a message to ensure that the intended meaning is expressed exactly, and that extraneous information is minimised.

- Reprocessability

Reprocessability refers to the extent to which a message can be re-examined or processed again within the context of the communication event, also termed "externally recorded memory". 


\begin{tabular}{|c|c|c|c|c|c|}
\hline & 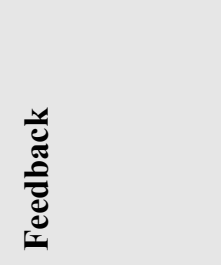 & 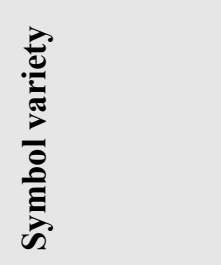 & 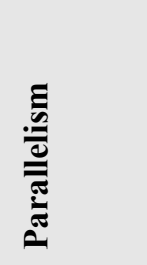 & 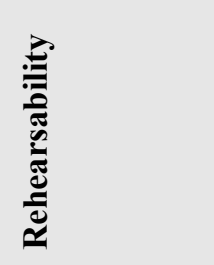 & 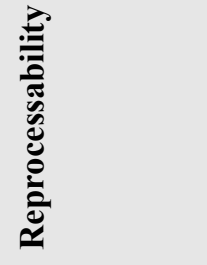 \\
\hline Face-to-face & High & Low-high & Low & Low & Low \\
\hline Video conference & Medium-high & Low-high & Low & Low & Low \\
\hline Telephone & Medium & Low & Low & Low & Low \\
\hline Written mail & Low & Low-medium & High & High & High \\
\hline Voice mail & Low & Low & Low & Low-medium & High \\
\hline Electronic mail & Low-medium & Low-high & Medium & High & High \\
\hline Online chat & Medium & Low-medium & Medium & Low-medium & Low-medium \\
\hline $\begin{array}{l}\text { Asynchronous } \\
\text { groupware }\end{array}$ & Low & Low-high & High & High & High \\
\hline $\begin{array}{l}\text { Synchronous } \\
\text { groupware }\end{array}$ & Low-medium & Low-high & High & Medium-high & High \\
\hline
\end{tabular}

Table 33: Relative Trait Salience of Selected Media (Dennis \& Valacich, 1999; p. 3)

These media characteristics are important in understanding the effect of media use on the ability to communicate and process information. Moving away from the concern of social presence that dominated media richness theory, the inclusion of parallelism, rehearsability and reprocessability show that while still considering the role of social presence in conveying information, MST is also concerned with information processability. This is especially important to the current study as Managers are faced with a large amount of information from varying sources that they must process in order to improve their understanding of a practice-related issue and inform their practice.

Within Table 33 some media have a range of capabilities, for example electronic mail has a feedback capability of 'low-medium'. This is because the capability can differ depending on how the email system is configured for a particular group undertaking a particular task within a particular organisational environment. Media can be seen to possess different capabilities, each of which is more or less effective to support a particular communications process within a given situation. It is apparent then, that no one medium has the highest values on all dimensions, and therefore none is the 'richest' or the 'best' as asserted by media richness theory. The media most appropriate to a particular situation is that which best provides the set of capabilities needed by that situation: the individuals, the task and social context within which they interact.

Following this, matching the five media characteristics of feedback, symbol variety, parallelism, rehearsability and reprocessability to the communication processes of conveyance and convergence that are required for a particular task is the key to successful media use. This is the underlying premise of MST. Dennis \& Valacich defined high synchronicity as that which has a high level of feedback and a low level of parallelism, and low synchronicity as that which has a low level of feedback and high parallelism. There is therefore not one 'richest' media as in media richness 
theory, as different set of media can provide high (or low) synchronicity depending on the nature of the task and the configurability of the media itself.

Assessing the ability of the media capabilities to support the communications processes of conveyance and convergence, Dennis \& Valacich (1999) drew several conclusions that are summarised here:

- Conveyance: low media synchronicity and higher reprocessability is preferred

- Convergence: high media synchronicity is preferred

- For both conveyance and convergence:

- A medium's symbol variety will only affect performance when a needed symbol set is unavailable

- Use of media that provide higher rehearsability will lead to better performance

Groups undertaking synchronous tasks (and to a lesser extent asynchronous tasks) need to select from a set of media that the group uses at different times when undertaking tasks, dependent on the prominent communications process. Furthering this point, Dennis \& Valacich found that most tasks require conveyance and convergence, and the media that is best matched to one process is often not ideal for the other. Therefore media switching was deemed most appropriate for groups that are performing synchronous tasks21 (Dennis \& Valacich, 1999).

${ }^{21}$ This is discussed further in sections 5.2.2 and 6.2.2 where MST is applied to specify the technology choices most appropriate in supporting peer consulting within AC and CI (respectively). 


\section{Appendix E - Hewson Triangle}

This Appendix illustrates the Hewson Triangle framework, parts of which AC used to structure their online peer consulting sessions of the Engaging stage. This is a supervision framework for analysing cases that moves through identifying and discussing a series of twelve points organised into three categories: client or problem; clinician; the relationship between them. The framework assists counsellors in professional supervision sessions to analyse key elements of a particular client case and/or issue, their clinical role and past and future actions, and the relationship between clinician and client.

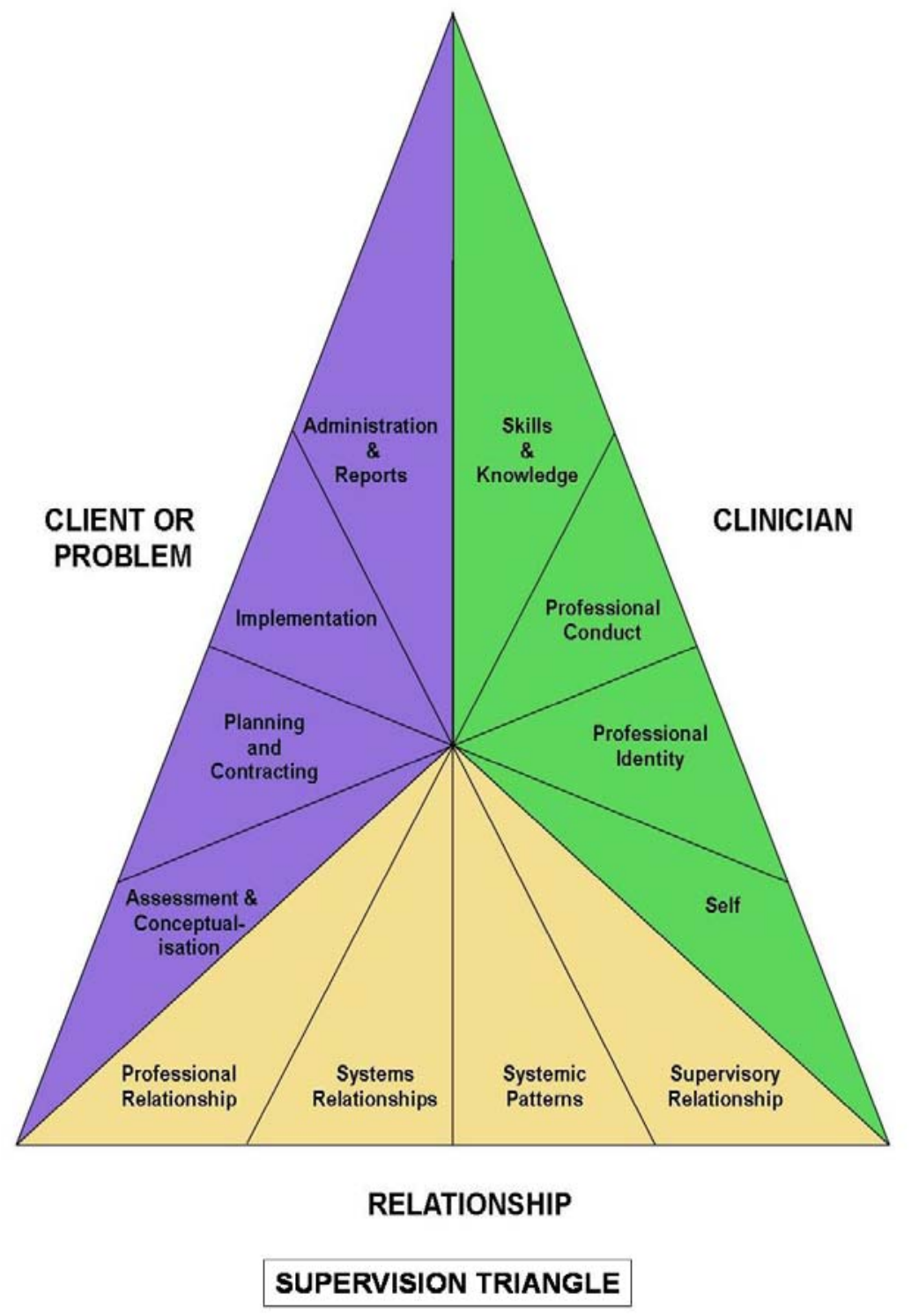

Hewson, D (2002) Supervision of psychologists: a supervision triangle.

In M.McMahon \& W. Patton (eds), Supervision in the Helping Professions: A Practical Approach. Pearson Education, Frenchs Forest, Australia.

Figure 20: A Supervision Triangle (Hewson, 2002). 
Additional detail is provided in Figure 21, which delineates elements of the Hewson Supervision Structure.

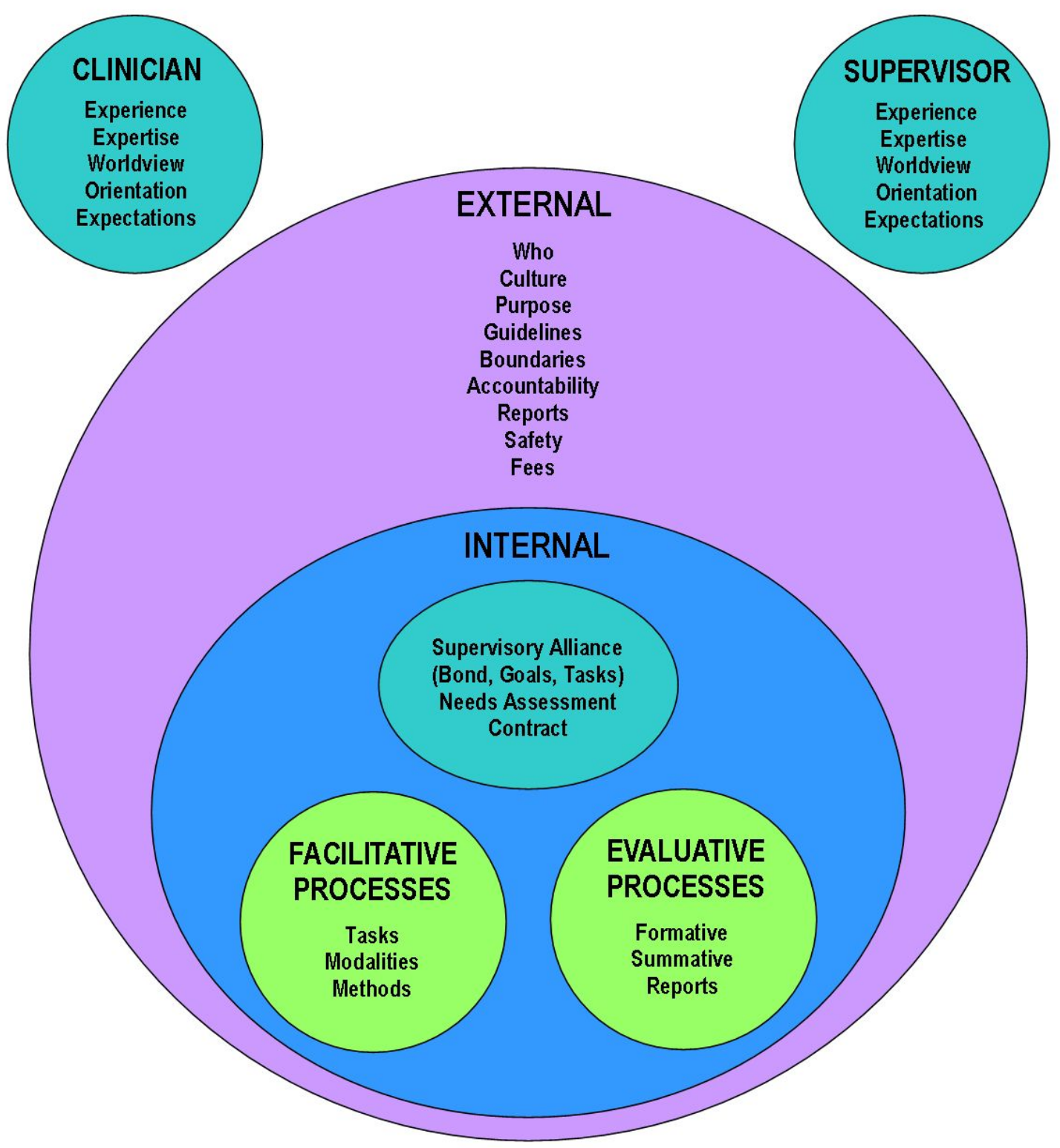

Figure 21: Detail of Hewson Supervision Structure 


\section{Appendix F - Issues on which CI Peers Consult}

The Managers within CI are tasked with assisting other Managers and counsellors with a wide range of issues and problem situations on an ongoing basis. In addition to situations requiring peer consultation (as discussed throughout Chapter 6 and within Appendix F) some examples of the types of issues that Managers face are highlighted here.

- The presenting of an issue centred on a 2-location, Family Court case At times, a case may involve members of a family that are located at two (or on occasion more) different geographical locations. In such a situation, two (or more) counsellors from separate CI regions will work together to offer counselling services to the family. At times, problems or issues are encountered by the counselling staff (such as inadequate reports, disagreement over outcomes etc) that require the attention of the appropriate Managers. A Manager may need to ascertain the problem/issue faced by the counsellor in their region and then consult with the Manager from the other region(s) in order to resolve the situation at hand.

- The exchange of paperwork around a particular process A recent example of this type of situation is an audit that was carried out within CI. Managers were directly responsible for completing some audit requirements for the office(s) within their region. As many Managers did not have experience with this process, they needed to consult with peers, which resulted in the exchange of paperwork regarding a successful audit process.

- Issues arising on the introduction of a new process/practice $\mathrm{CI}$ is continually evolving as an organisation and this includes the counselling programs and practices that are undertaken by counsellors and overseen by Managers. When a counsellor is involved with running a program that he/she has not previously run, the Manager must ensure that the counsellor(s) involved have the training (knowledge and skills) and resources necessary. A Manager must also be prepared to answer any questions that the counsellor has during the running of the program and be on hand to debrief at the conclusion of the session. 


\section{Appendix G - Cl Scoping Document}

Appendix D includes the project scoping document that was generated as part of the Defining stage activity of developing the online peer consulting concept.

\section{Joint peer consulting project between Counselling Incorporated and Julie Abbott}

Julie Abbott is a PhD student within the School of Information Management at Victoria University, Wellington. Her research interest is in how technology can support people in the not-for-profit sector that are working at different geographical locations. Pak Yoong, who has helped students to undertake research in partnership with Counselling Incorporated in the past, supervises Julie. She will be continuing some of this past work as part of her $\mathrm{PhD}$.

Many of you would have asked for advice or assistance from other counsellors from time to time, and may even be involved in a group of counsellors that support and help each other when needed. Such a group of practitioners can be termed a 'community of practice'. When counsellors meet to share professional knowledge and practices that will help them in their work, they are undertaking what researchers such as Julie call 'peer consulting'. Peer consulting is one type of professional development activity that groups, including communities of practice, undertake.

Currently within Counselling Incorporated there are few opportunities for counsellors from around NZ to meet face-to-face to be involved in peer consulting sessions. As all counsellors face limitations in terms of their available time and finances, they cannot always gain the benefits of consulting with other counsellors.

Julie proposes the adoption of a simple online system to enable different groups of counsellors to discuss the practice-related problems and issues that are important to them and that will contribute to their professional development. With the support of technology, groups of practitioners could engage regularly over the Internet for peer consulting without the need to travel for face-to-face meetings. Regular connections could be made through this system between geographically separated counsellors where there were none, supporting the communications and collaborations that are needed for peer consulting to take place.

As part of this project, Julie will be working closely with various counsellors within Counselling Incorporated to get the pilot online peer consulting system up and running, and to test its usefulness in current work situations. This will mainly involve interviews with staff during the life of the project to gain their perspectives and experiences on this topic. 
The main benefit of this project will be the development of an alternative way to enable counsellors to undertake regular peer consulting sessions, no matter where in NZ they are. Counselling Incorporated may not only save time and financial resources, but the staff will benefit directly and immediately through being part of a community of practitioners. 


\section{Appendix H - The Nature of Cl's Peer Consulting Process}

Appendix $\mathrm{G}$ includes additional detail of the peer consulting process, developed through the Defining stage activity of mapping current peer consulting activities.

\section{The nature of the consultee process}

When faced with an issue or concern that they cannot resolve themselves a Manager would most often contact the National Manager of Professional Practice located in Wellington. Issues that would prompt this interaction were given as clinical issues and/or procedural or organisational issues.

When seeking advice from an internal or external peer, Managers generally know who has the knowledge and experience needed to engage in consultation and assist in improving their understanding of a presenting issue. They therefore have not had difficulty in identifying the best person to contact. This was reported to be because of the organisational knowledge that they have built up during their time with CI, and because of the contacts made and maintained through their managerial position.

The typical duration of a consultation in which a Manager is seeking advice varies, depending on the situation at hand, the level of urgency surrounding the consultation and by other events within CI at the particular time of the consultation. Duration of a consultation was reported to vary from five minutes, to one hour, to one day, to three days, to one week, to 'ongoing'. Furthermore, the part-time nature and different working hours of most positions within CI can cause delays within the consulting process that impact the overall duration of the consultation.

\section{The nature of the consultor process}

On occasion, a Manager will be required to give help or assistance to another Manager. An example of an issue that may prompt this interaction is issues surrounding the supervision of a peer. However, Managers most often give advice, or are consulted by the staff that work in the same or 'immediate' office. This consultation typically occurs outside of the formal supervision relationship that a Manager may have with staff and is therefore of an informal and often 'ad hoc' nature.

The staff within the wider area that a Manager is responsible for also frequently consult them. Examples of when a Manager gives advice were given as:

- Confirmation of an CI policy surrounding a particular case issue

- The running of a program that is new to CI

- The handling of requests for information from other agencies about CI cases 
As with the description of seeking advice or help, they also gives advice or help to the relevant Area Manager quite frequently, though less often than to other staff and usually based around managerial issues as opposed to clinical issues.

Managers reported that they did not often need to contact staffs (excluding Managers) from outside of their particular area to give assistance, except perhaps during exceptional circumstances such as the sudden unexpected absence of a Manager. Instead of direct contact with staff, a Manager would typically contact the other relevant Manager, who then contacts the staff member in question directly to discuss the presenting issue.

The frequency with which a peer consults a Manager varies greatly, although all Managers did state that being consulted is a regular occurrence. The degree of regularity varies from multiple times per day, to daily, to weekly. Again, the typical duration of a consultation in which they give advice or help to a peer varies, from five minutes to up to one week. The frequency and duration of a consultation are dependent on the nature of the issue, the time urgency involved (although this is usually low) and the other events going on within the organisation at the time.

Managers experienced some difficulties when being consulted by a peer. These difficulties essentially involve communication and the 'fractured' work hours of regional staff and Managers was the main contributing factor. Some examples of difficulties were given as:

- Connecting Managers and staff, and connecting staff to other staff

- Responding to requests for help in a timely manner

- Being accessible and available to regional staff regularly

\section{Additional peer consulting issues}

It was most common for peer consulting to take place in an informal, ad hoc basis between Managers and/or staff from within the same office. This was supported by a culture in which a high level of trust and trusting relationships are essential.

In addition, there are two national meetings per year - the Manager meeting and the Manager and area manager meeting - where all Managers meet face-to-face in Wellington to discuss organisational and practice related issues. One participant described these two national meetings as "an opportunity to discuss practice related issues with a group of peers" (P12). This same participant viewed these meetings as a supportive environment where networking could take place.

In addition to the two national meetings, regional teleconference meetings (up to three per year) are also held within Northern, Central and Southern areas. 


\section{Appendix I - Process Maps Addendum}

This research revealed that the consultee will often be consulting with one or more peers, not only one. Therefore, the consultee and consultor process mappings presented here extend previous research further by incorporating not only the individual, or one-to-one perspective of peer consulting, but also the one-to-many and many-to-many perspectives. This occurs at the step "consultation takes place" (Figs. 2 \& 3) This is also reflected in the amendments made to the titles of some steps, changing from the individual 'person' to the individual or multiple 'person(s)' (Steps 2 and 3 of the Consultee diagram). The addition of the step 'set up consulting group' (step 8 of the Consultor process) further reflects the inclusion of the group perspective (one-to-many or many-to-many) of the peer consulting process.

Note also that the relevant parties to a consultation may be from within or outside of CI. Thus, during the steps 'consultation takes place', 'set up consulting group' or 'discussion with a $3^{\text {rd }}$ party' one or more persons may be external to CI. 


\section{Appendix J - Cl Peer Consulting Session Plan}

This appendix includes the series structure document that was generated as part of the Structuring stage at CI.

\section{Peer Consulting Session Structure}

The purpose of this document is to introduce and outline the structure of a series of peer consulting sessions to be held at Counselling Incorporated during 2005-2006. These peer consulting sessions are intended as an opportunity for Managers to connect and engage in discussion on current practice related issues. As such, the peer consulting sessions will offer value from two perspectives: 1) the topics for discussions are related to current practice issues, so individual and group learning on these practice areas can take place. 2) to explore the possibilities of using different systems tools to support the ongoing professional development of Managers at Counselling Incorporated.

\section{Letting people know about a session}

SMa and Julie will let Managers know of an upcoming session in two ways: through email and also via conference calls.22 Managers will know about each peer consulting session at least one week before the session commences, in order to allow enough time for any necessary scheduling and preparation to take place.

\section{Timeframe for sessions}

Each peer consulting session will be of approximately two weeks duration. As most Managers hold part time positions a period of two weeks has been set to ensure that each Manager has an opportunity to engage with others during a session, at a time(s) that is convenient to them. The time period between each two week peer consulting session will be three weeks. During this time SMa, SMb and Julie will work on other activities to conclude a session and set up for the next session.

\section{Session themes}

Each peer consulting session will be organised around a particular professional development theme related directly to the professional practice of Managers. A set of initial topics has been determined:

- Working with children

- Working with youth

- Performance management

- Couples counselling

${ }^{22}$ Conference calls that SMa has with each regional practice manager group. 
Nearer to the time of each peer consulting session there may be other topics that present themselves as being current and relevant, and of value for discussion by Managers. It is anticipated that such topics may be substituted for those on this initial list.

\section{Session resources}

Introducing an article, passage (statement, quote etc) or case situation for discussion during a peer consulting session will help to focus the session and add some structure. For each separate topic the resources for discussion may vary, depending on the nature of that topic and the appropriate resources available / sourced. SMa and SMb will decide on the resources to be used for each session, and these will be available to Managers at least a week before each session takes place.

The 'technical' resources to support the peer consulting sessions may also change as the session schedule progresses. The discussion forums and document repository area of the Blackboard system will be used for the initial sessions. Based on feedback gained from Managers on their use of Blackboard, a decision will be made on the use of the audio system 'Chatterbox' for later peer consulting sessions.

\section{Output of sessions}

A summary of key discussion points and issues will be generated at the conclusion of each peer consulting session. With feedback from SMa and SMb, Julie will be responsible for collating the discussions that take place during each peer consulting session and forming them into a document.

Each summary document will be given to Managers via email and also posted on the Blackboard system. These documents will combine to form a set of 'learning outcomes' of the series of peer consulting sessions.

\section{Feedback on sessions}

To gather the Managers' experiences of participating in the peer consulting sessions, SMa and Julie will ask for feedback in three main areas:

- Their perceived value of the peer consulting sessions

- Their experience of changes to the wider peer consulting process

- Their use of the system(s) supporting the sessions

It is intended that this feedback can be gained from Managers through discussions during conference calls. As interesting feedback is given as the peer consulting sessions progress, interviews with Managers may be held to gather additional insights on points that arise. In addition, useful feedback will be given to Managers on the peer consulting sessions as they progress. 


\section{Time line for sessions}

A time line for what the session period is likely to look like, with activities before and after the actual peer consulting session, is included below.

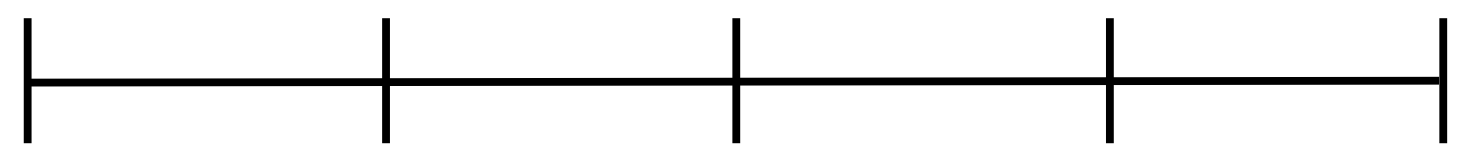

Week 1

Regional practice
managers are
informed of the
upcoming session
Session resources
(discussion case
or question) are
given to
participants via
email and
Blackboard
The Blackboard
discussion forum
is set up

Regional practice managers are informed of the

is set up
Week 2

The peer consulting session begins:

The discussion forum is activated and discussions begin

The Blackboard system is monitored and any needed support is given to participants
Week 3

Week 4
The session continues:

Discussions continue until the end of the week, and any needed Blackboard support is given

At the end of the week the session concludes, and the discussion forum is 'closed'
During weeks 4 \& 5 the discussion points are collated, checked and then given to regional practice managers via email and Blackboard

Participants are asked about their experience of participating in the session, and the value of the session

Preparation is made for the next session, including confirmation of the topic and resources, and setting up of the support system(s) 


\section{References}

Adams, D., Nelson, R. \& Todd, P. (1992). "Perceived usefulness, ease of use, and usage of information technology: a replication." MIS Quarterly archive 16 (2): 227 247.

Ahuja, G. (2000). "Collaboration networks, structural holes, and innovation: A longitudinal study." Administrative Science Quarterly, 45(3): 425-455.

Akkerman, S., Petter, A. \& de Laat, M. (2008). "Organising communities of practice: facilitating emergence." Journal of Workplace Learning, 20(6): 383-399.

Allen, N. (2003). "Using email and the web to acquire and cultivate donors." Nonprofit World, 21(1): 27-28.

Al-Qirim, N. (2007). "E-Commerce Adoption in Small Business: Cases from New Zealand." Journal of Information Technologhy Case and Application Reasearch, 9(2): $28-57$.

Ariss, S., Raghunathan, T. \&Kunnathar, A. (2000). "Factors affecting the adoption of advanced manufacturing technology in small firms." S.A.M. Advanced Management Journal, 65(2): 14-23.

Avison, D., Baskerville, R. \& Myers, M. (2001). "Controlling action research projects.” Information Technology \& People, 14(1): 28-45.

Barker-Ruchti, N. (2002). "A study journey: A useful example of action research." Journal of Physical Education New Zealand, 35(1): 12-19.

Barrett, M. (2001). "A stakeholder approach to responsiveness and accountability in non-profit organisations.” Social Policy Journal of New Zealand, 17: 36-51.

Baskerville, R. (1999). "Investigating information systems with action research." Communications of the AIS, 2(19): 1-23.

Baskerville, R. (2001). "Conducting action research: High risk and high reward in theory and practice," in Trauth, E. (Ed), Qualitative Research in IS: Issues and Trends, Idea Group Publishing, London: 192-217.

Baskerville, R. \& Pries-Heje, J. (1999). "Grounded action research: A method for understanding IT in practice.” Accounting, Management and Information Technology, 9: $1-23$.

Baskerville, R. \& Wood-Harper, A. (1996). “A critical perspective on action research as a method for information systems research." Journal of Information Technology, 11: $235-246$. 
Baskerville, R. \& Wood-Harper, A. (1998). "Diversity in information systems action research methods." European Journal of Information Systems, 7: 90-107.

Bernard, R., de Rubalcava, B. \& St-Pierre, D. (2000). "Collaborative online distance learning: Issues for future practice and research.” Distance Education, 21(2): 260-277.

Benbasat, I., Goldstein, D. \& Mead, M. (1987). The Case Research Strategy in Studies of Information Systems. MIS Quarterly, 11(3): 369-386.

Blackboard Incorporated. (2009). Blackboard Lean Powers Watson's Conintuous Learning Environment. Published Case Study, Sourced from:

http://www.blackboard.com/CMSPages/GetFile.aspx?guid=ba4dd6c7-a814-4bd08997-816ded1ab3c2

Bostrom, R. \& Clawson, V. (1992). "The role of the facilitator in computer-supported environments." Unpublished doctoral thesis.

Boudreau, M. \& Robey, D. (2005). "Enacting Integrated Information Technology: A Human Agency Perspective.” Organization Science, 16(1): 3-18.

Braa, R. \& Vidgen, K. (1999). "Interpretation, intervention, and reduction in the organisational laboratory: A framework for in-context information systems research." Accounting, Management and Information Technology, 9 (1): 1-21.

Briggs, R., Adkins, M., Mittleman, D. \& Kruse, J. (1999). "A technology transition model derived from field investigation of GSS use abroad the U.S.S. CORONADO." Journal of Management Information Systems, 15(3): 151-195.

Briggs, R., Nunamaker, J. \& Tobey, D. (2001). "The technology transition model: A key to self-sustaining and growing communities of GSS users." Proceedings of the 34th Hawaii International Conference on Systems Sciences, p. 1-9.

Brown, M. (2001). "Learning about leadership from community organizers." The Journal for Quality and Participation, 24(3): 50-55.

Bruce, B. \& Easley, J. (2000). "Emerging communities of practice: Collaboration and communication in action research." Educational Action Research, 8(2): 243-259.

Bussell, H. \& Forbes, D. (2002). "Understanding the volunteer market: The what, where, who and why of volunteering." International Journal of Nonprofit and Voluntary Sector Marketing, 7(3): 244-257.

Cecez-Kecmanovic, D. (2002). "Enabling knowledge sharing: New challenges for information systems." Proceedings of the Thirteenth Australian Conference on Information Systems, December 4-6: 61-73.

Chaisson, M. \& Dexter, A. (2001). "System development conflict the use of an information systems prototyping method of action research: Implications for practice and research." Information Technology \& People, 14(1): 91-108. 
Chambers, T. \& Parker, C. (2000). "Factors motivating and inhibiting the use of web commerce by rural small businesses." Proceedings of the Eleventh Australian Conference on Information Systems, December 4-6: p. 1-12.

Chang, H. \& Johnson, J. (2001). "Communications networks as predictors of organizational members' media choices.” Western Journal of Communication, 65(4): 349-369.

Chau, S. \& Turner, P. (2002). "An exploration of factors that influence the ability of small and medium sized enterprises to engage in electronic commerce: Preliminary findings from 34 Australian case studies." Proceedings of the Thirteenth Australian Conference on Information Systems, December 4-6: p. 209-217.

Chisholm, R. \& Elden, M. (1993). "Features of an emerging action research." Human Relations, 46(2): 275-295.

Cockburn, L. \& Trentham, B. (2002). "Participatory action research: Integrating community occupational therapy practice and research." The Canadian Journal of Occupational Therapy, 69(1): 20-32.

Coetzer, A. \& Perry, M. (2008). "Factors influencing employee learning in small businesses." Education and Training, 50(8/9): 648-660.

Conrad, D. (2005). "Building and Maintaining Community in Cohort-Based Online Learning." Journal of Distance Education, 20(1): 1-20.

Cragg, P. (2008). "Identifying key information systems competencies in small firms." Total Quality Management \& Business Excellence, 19 (1-2): 29-35.

Cragg, P., Mills, A.M. and Suraweera, T. (2010). "Understanding IT Management in SMEs." Electronic Journal of Information Systems Evaluation, 13(1): 27-34.

Cragg, P., Tagliavini, M., Mills, A. (2007). "Evaluating the Alignment of IT with Business Processes in SMEs." University of Southern Queensland, Toowoomba, Australia: 18th Australasian Conference on Information Systems (ACIS 2007), 5-7 Dec 2007. Proceedings of the 18th Australasian Conference on Information Systems, p. 38-48.

Creswell, J. (1994). Research Design: Qualitative and Quantitative Approaches. Beverley Hills: Sage Publications.

Creswell, J. (1998). Qualitative Inquiry and Research Design: Choosing Among Five Traditions. California: Sage Publications.

Daft, R. L. \& Lengel. (1986). “Organizational information requirments, media richness and structural design." Management Science 33(5): 554-571. 
Davison, R. (2001). "GSS and action research in the Hong Kong police." Information Technology \& People, 14(1): 60-77.

De Cagna. J., (2001). "Interview - Tending the garden of knowledge: A look at communities of practice with Etienne Wenger." Information Outlook, 5(7): 6-12.

DeLone, W. \& McLean, E. (2003). "The DeLone \& McLean Model of Information Systems Success: A Ten-Year Update.” Journal of Management Information Systems, 19(4): 9-30.

DeLuca, D. (2006). "Virtual teams in and out of Synchronicity." Information Technology \& People, 19 (4): 323-344.

Denison, T., Hardy, G., Johanson, G., Stillman, L. \&Schauder, D. (2002).

"Community networks: Identities, taxonomies and evaluations."Proceedings of Electronic Networking 2002 - Building Community, July 3-5: p. 1-17.

Dennis, A. Valacich, J. (1999). "Rethinking Media Richness: Towards a Theory of Media Synchronicity." In Proceedings of the 32nd Hawaii International Conference on System Sciences, January 05-08, 1999.

Dennis, A., Fuller, R. \& Valacich, J. (2008). "Media, tasks, and communication processes: a theory of media synchronicity." MIS Quarterly, 32(3): 575-600.

Dery, K., Hall, R. \& Wailes, N. (2006). “ERPS as 'technologies-in-practice': social construction, materiality and the role of organisational factors". New Technology, Work and Employment, 21(3): 229 - 241.

DeSanctis, G. \& Poole, M. (1994). "Capturing the complexity in advanced technology use: Adaptive Structuration Theory”. Organisation Science, 5(2): 121 - 147.

Desouza, K. \& Awazu, Y. (2006). "Knowledge management at SMEs: five peculiarities." Journal of Knowledge Management,10(1): 32-43.

Einstein, W. \& Humphreys, J. (2002). "The changing face of leadership: The influence of information technology": 1-26.

Eisenhart, M. (1998) "On the subject of interpretive reviews." Review of Educational Research, 68(4): 391-399.

Eisen, M. (2001). "Peer-based professional development viewed through the lens of transformative learning." Holistic Nursing Practice, 16(1): 30-42.

Elden, M. \& Chisholm, R. (1993). "Emerging varieties of action research." Human Relations, 46(2):121-141.

Ellison, N. (1999). "Social impacts: new perspectives on telework." Social Science Computer Review, 17(3): 338-356. 
Ercetin, S. (2002). “Action research...organisational intelligence...curriculum development...” Educational Research Quarterly, 26(1): 41-46.

Facebook (2010). Facebook Factsheet. Retrieved from:

http://www.facebook.com/press/info.php?factsheet

Fink, D. \& Disterer, G. (2006). "International case studies: To what extent is ICT infused into the operations of SMEs?" Journal of Enterprise information Management, 19(6): 608-624.

Franke, U. (2001). "The concept of virtual web organisations and its implications on changing market conditions." Electronic Journal of Organizational Virtualness, 4(1): 43- 64 .

Franke, U. \& Hickmann, B. (1999). "Is the net-broker an entrepreneur? What role does the net-broker play in virtual webs and virtual corporations?" Virtual Organisation Net, 1(1): 120-138.

Fulk, J. (1993). "Social Construction of Communication Technology.” Academy of Management Journal, 36(5): 921-950.

Garcia, L. (1993). “A new role for Government in standard setting?" Standard View, 1(2): 2-10.

Green, M. \& Cifuentes, L. (2008). “An Exploration of Online Environments Supporting Follow-Up to Face-to-Face Professional Development.” Journal of Technology and Teacher Education 16(3): 283-306.

Gibb, A. (2000). "SME policy, academic research and the growth of ignorance, mythical concepts, myths, assumptions, rituals and confusions." International Small Business Journal, 18(3): 13-35.

Giddens, A. (1984). The constitution of society: Outline of the theory of structuration. Berkeley, University of California Press.

Gittell, M., Ortega-Bustamante, I. \& Steffy, T. (2000). "Social capital and social change: Women's community activism." Urban Affairs Review, 36(2): 123-147.

Gnyawali, D. \&Madhavan, R. (2001). "Cooperative networks and competitive dynamics: A structural embeddedness perspective." The Academy of Management Review, 26(3): 431-445.

Gongla, P. \&Rizzuto, C. (2001). "Evolving communities of practice: IBM Global Services experience.” IBM Systems Journal, 404: 842-862.

Grandhi, S., Jones, Q., Chivakula, K. \& Patten, K. (2003). "Media Switching and Media Integration: An Examination of Instant Messaging and IP-Calling Practices." Ninth Americas Conference on Information Systems. 2142-2147 
Haggerty, N. (2000). "Understanding the link between IT project manager skills and project success: Research in progress." Communications of the ACM, 1(58): 192-195.

Hammersley, M. (1990). "What's wrong with ethnography? The myth of theoretical description." Sociology: the Journal of the British Sociological Association 24(4): 597-615.

Harris, M. \& Harris, J. (2002). "Achieving organisational collaboration in the nonprofit sector: An action research approach." Organisational Development Journal, 20(1): 28-35.

Haythornthwaite, C. (2001). "Tie strength and the impact of new media." Proceedings of the $34^{\text {th }}$ Hawaii International Conference on System Sciences, Unpublished

Hildreth, P., Kimble, C. \& Wright, P. (2000). "Communities of practice in the distributed international environment." Journal of Knowledge Management, 4(1): 2738 .

Holbeche, L. (1996). "Peer mentoring: The challenges and opportunities" Career Development International, 1(7): 24-27.

Huxham, C. \& Vangan, S. (2000). "Leadership in the shaping and implementation of collaboration agendas: How things happen in a (not quite) joined-up world." Academy of Management Journal, 43(6): 1159-1175.

Igbaria, M., Zinnatelli, N., Cragg, P. \&Cavaye, A. (1997). "Personal computing acceptance in small firms: A structural equation model." MIS Quarterly, 21(3): 279305.

Inram, A. \& Hathorn, L. (2004). "Methods for Analyzing Collaboration in online Communications." In Roberts, T. (Ed.). Online Collaborative Learning: Theory and Practice (pp 215-309). Information Science Publishing, London.

Jagers, H., Jansen, W. \& Steenbakkers, W. (1998). "Characteristics of virtual organisations.” Proceedings of VoNet - Workshop, April $27-28$.

Jansen, W., Steenbakkers, W. \&Jagers, H. (1999). "Electronic commerce and virtual organisations.” Electronic Journal of Organisational Virtualness, 1(1): 54-68.

Jones, N. \& Robinson, G. (1997). "Do organisations manage continuing professional development?" The journal of Management Development, 16(3): 197-204.

Jonsson, S. (1991). "Action research,” in Nissen, H., Klein, H. \& Hirscheim, R. (eds), Information Systems Research: Contemporary approaches \& emergent traditions, Elsevier Science Publishers, New York: 371-396.

Kayworth, T. \& Leidner, D. (2002). "Leadership effectiveness in global virtual teams." Journal of Management Information Systems, 18(3): 7-40. 
Keats, D. (2003). "Towards an online peer consulting system for Counselling Incorporated." Unpublished INFO 408 Research Project. Victoria University of Wellington, New Zealand.

Kehrwald, B. (2008). "Understanding social presence in text-based online learning environments.” Distance Education, 29 (1): 89-106.

Kimble, C. \& Hildreth, P. (2005). "Dualities, distributed communities of practice and knowledge management." Journal of Knowledge Management, 9(4): 102-113.

Kimble, C., Li, F. \& Barlow, A. (2000). 'Effective virtual teams through communities of practice.” Management Science, (9): 1-15.

Klein, H. \& Myers, M. (1999). "A set of principles for conducting and evaluating interpretive field studies in information systems.” MIS Quarterley, 23(1): 67-88.

Klein, H. \& Myers, M. (2001). "A classification scheme for interpretive research in information systems," in Trauth, E. (ed), Qualitative Research in IS: Issues and Trends, Idea Group Publishing, London: 218-239.

Kock, N. \& Lau, F. (2001). "Information systems action research: Serving two demanding masters." Information Technology \& People, 14(1): 6-11.

Lanam, W. (2001). "Running the gauntlet.” Enterprise Systems Journal, 16(1): 20-24.

Lau, F. (1997). "A Review on the Use of Action Research in Information Systems Studies," In A.S. Lee, J. Liebenau and J.I. DeGross (Eds.). Information Systems and Qualitative Research (pp. 31-68). Chapman and Hall, London.

Lau, F. (1999). "Toward a framework for action research in information systems studies.” Information Technology \& People, 12(2): 148-164.

Leedy, P. (1997). Practical Research: Planning and Design. New Jersey: Prentice Hall.

Leh, A. (2002). "Action research on the changing roles of the instructors and the learners." TechTrends, 46(5): 44-49.

Lengel, R.H. \& Daft, R.L. (1988). "The Selection of Communication Media as an Executive Skill.” Academy of Management Executive, 2(3), 225-232

Leslie, K., Lindsay, V., Mullings, H. \&Salkeld, N. (1999). "Why companies have much to learn from charities: VIEWPOINT MCKINSEY ON LESSONS FROM THE VOLUNTARY SECTOR: The voluntary sector and commercial world have much in common - and charities are further ahead in handling change." Financial Times: 1618.

Lesser, E. \&Prusak, L. (1999). White Paper "Communities of practice, social capital and organizational change.” IBM Institute for Knowledge Management, Cambridge, MA. 
Lewis, K., Massey, C., Ashby, M., Coetzer, A. \& Harris, C. (2007) "Business assistance for SMEs: New Zealand owner-managers make their assessment." Journal of Small Bussiness and Enterprise Development, 14(4): 551-566

Lewis, K., Massey and Harris, C. (2007). "Learning by ding: six dimensions f complexity in researching SME's." Qualitative Research in Accounting \& Management, 4(2): 151-163.

Lock, J. (2006). 'A New Image: Online Communities to Facilitate Teacher Professional Development." Journal of Technology and Teacher Education, 14(4): 663-678.

Locke, S. (2004). "ICT Adoption and SME Growth in New Zealand" Journal of American Academy of Business, Cambridge, 4(1/2): 93-102.

Majchrzak, A., Rice, RE., Malhotra, A., King, N. \& Ba, S. (2000). "Technology Adaption: The Case of A Copmuter-Supported Inter-organizational Virtual Team." MIS Quarterly, 24(4): 569-600.

Marquardt, M. (2000). “Action learning and leadership.” The Learning Organization, 7(5): 233-240.

Massey, C. (2004). "Employee practices in New Zealand SMEs.” Employee Relations, 26(1): 94-105.

Meister, D. \& Gronksi, C. (2007). "Action research in a virtual setting: Cautions from a failed project." In Kock, L. (Ed.), Information Systems Action Research: An Applied View of Emerging Concepts and Methods (pp. 217-240). Springer Science + Business Media, USA.

Massa, S. \& Testa, S. (2005). "Data warehouse-in-practice: exploring the functions of expectations in organizational outcomes". Information \& Management, 42: 709-718.

Maznevski, M. \& Chudoba, K. (2000). "Bridging Space Over Time: Global Virtual Team Dynamics and Effectiveness.” Organization Science, 11(5), 473-492.

McCotter, S. (2001). "The journey of a beginning researcher.” The Qualitative Report, $\underline{6}(2): 1-21$.

McDermott, R. (2000). "Community development as a natural step: Five stages of community development." Knowledge Management Review, 3(5): 16-19.

McKay, J. \& Marshall, P. (1999). “2 x $6=12$, or does it equal action research?” Proceedings of the 10th Australasian Conference on Information Systems, 1999: 597 609.

McKay, J. \& Marshall, P. (2001). "The dual imperatives of action research." Information Technology \& People, 14(1): 46-59. 
McKay, J. \& Marshall, P. (2007) "Driven by two masters, serving both: The interplay of problem solving and research in information systemsi" In Kock, L. (Ed.), Information Systems Action Research: An Applied View of Emerging Concepts and Methods (pp. 131-158). Springer Science + Business Media. USA.

Miles, M. \& Huberman, A. (1984). Qualitative Data Analysis: A Sourcebook of New Methods. CA: Sage Publications. USA.

Ministry of Economic Development. (2002). SMEs in New Zealand: Structure and Dynamics. The Ministry of Economic Development SME Policy documents. Available at: http://www.med.govt.nz/irdev/ind_dev/smes2002/smes2002-02.html

Ministry if Economic Development. (2007). SMEs in New Zealand: Structure and Dynamics. The Ministry of Economic Development SME Policy documents. Available at: http://www.med.govt.nz/irdev/ind_dev/smes2002/smes2002-02.html

Ministry of Economic Development. (2009). SMEs in New Zealand: Structure and Dynamics. The Ministry of Economic Development SME Policy documents. Available at: http://www.med.govt.nz/irdev/ind_dev/smes2002/smes2002-02.html

Ministry of Social Policy. (2001). "Communities and Government: Potential for partnership, whakatopuwakakaaro." Community Policy Team. Available at http://www.msd.govt.nz/documents/work-areas/ocvs/communities-potential-partnershipwhakatoopu-whakaaro-full-report.pdf

Miranda, S.M. \& Bostrom, R.P. (1993-1994). "The impact of group support systems on group conflict and conflict management." Journal of Management Information Systems, 10(3): 63-95.

Miranda, S.M. \& Bostrom, R.P. (1999). "Meeting facilitation: process versus content interventions." Journal of Management Information Systems, 15(4): 89-114.

Moore, J. \&Barab, S. (2002). "The inquiry learning forum: A community of practice approach to online professional development." TechTrends, 46(3): 44-49.

Morris, D. (1999). "Recent cases. Commentary. Volunteering and unemployment status." The Industrial Law Journal, 28(3): 249-268.

Morrison, B. \& Lilford, R. (2001). "How can action research apply to health services?" Qualitative Health Research, 11(4): 436-449.

Mumford, E. (2001). “Advice for an action researcher." Information Technology \& People, 14(1): 12-27.

Munkvold, B. (2002). "Organisational implementation of collaboration technologies: An integrative review." Proceedings of the Thirteenth Australian Conference on Information Systems, December 4-6: p. 355-367. 
Myers, M. (1997). "Qualitative research in information systems.” MIS Quarterly, 21(2): 241-242. MISQ Discovery, archival version.

Nonaka, I., Toyama, R. \& Konno, N. (2000). "SECI, ba and leadership: A unified model of dynamic knowledge creation." Long Range Planning, 33(1): 5-34.

Nonaka, I., von Krogh, G. \& Voelpel, S. (2006). "Organisational Knowledge Creation Theory: Evolutionary Paths and Future Advances." Organization Studies, 27(8): 1179-1208.

Nosek, J. (2007) "Insider as action researcher.” In Kock, L. (Ed.), Information Systems Action Research: An Applied View of Emerging Concepts and Methods (pp. 405-419). Springer Science + Business Media, USA.

Olesen, K. \& Myers, M. (1999). "Trying to improve communication and collaboration with information technology: An action research project which failed." Information Technology \& People, 12(4): 317-328.

Orlikowski, W. (1992). "The Duality of Technology: Rethinking the Concept of Technology in Organizations.” Organization Science, 3(3): Focused Issue: 398-427.

Orlikowski, W. (1995). "Action and artifact: The structuring of technologies-in-use." Retrieved 11th March, 2007, from http://hdl.handle.net/1721.1/2600.

Orlikowski, W. (2000). "Using technology and constituting structures: A practice lens for studying technology in organisations." Organization Science, 11(4): 404-428.

Orlikowski, W. \& Barley, (2001). "Technology and institutions: what can research on information technology and research on organizations learn from each other?" MISQ; Management Information Systems, 25(2): 145-165.

Orlikowski, W. \& Baroudi, J. (1991) "Studying information technology in organisations: Research approaches and assumptions." Information Systems Research, 2(1): $1-28$.

Paine, C., Reips, U., Stieger, S., Joinson, A., \& Buchanan, T. (2007). "Internet users' perceptions of 'privacy concerns' and 'privacy actions'." International Journal of Human-Computer Studies, 65(6): 526-536.

Peck, H. \&Juttner, U. (2000). "Strategy and relationships: Defining the interface in supply chain contexts." The International Journal of Logistics Management, 11(2): $33-44$.

Piggot-Irvine, E. (1996). "Reflection: The key to change in teaching action research." Action Learning and Action Research Journal, 1(2): 3-12.

Pozzebon, M., Diniz, E. \& Jayo, M. (2009) “Adapting the Structurationist View of Technology for Studies at the Community/Societal Levels." In Dwivedi, Y.K, Lal, B., 
Williams, M.D., Schneberger, S.L., and Wade, M.R. (Eds.). Handbook of Research on Contemporary Theoretical Models in Information Systems (pp. 18-33).

Information Science Reference. USA.

Prakash, A. \& De, R. (2007). "Enactment of Technology Structures in ICT4D Projects: A Study of Computerization of Land Records in India", 11th Pacific-Asia Conference on Information Systems, Auckland, New Zealand, 3-6 July, 2007:1-13

Putnam, R. (1993). "The prosperous community: Social capital and public life." The American Prospect, 13: 1-12.

Quadus, M. \& Xu, J. (2002). "Factors of adoption and diffusion of knowledge management systems in Australia: A structural equation modelling approach." Proceedings of the Thirteenth Australasian Conference on Information Systems, December 4-6: 75-86.

Ramsey, E. \& McCole, P. (2005). "E-business in professional SMEs: the case of New Zealand." Journal of Small Business and Enterprise Development, 12(4): 528-544.

Rapoport, R. (1970). “Three dilemmas in action research.” Human Relations, 23 (6): 499-513.

Riverin, S. \& Stacey, E. (2008). "Sustaining an Online Community of Practice: A Case Study." Journal of Distance Education, 22(2) 43: 58.

Robinson, D. (2001). "Social capital and voluntary activity: Giving and sharing in Maori and non-Maori society." Social Policy Journal of New Zealand, 17: 52-71.

Rock, T. \& Levin, B. (2002). "Collaborative action research projects: Enhancing preservice teacher development in professional development schools." Teacher Education Quarterly, 29(1): 7-18.

Rogers, E. M. (1995). Diffusion of innovations, 4th edition. The Free Press, New York.

Russell, K. (2001). "What is wilderness therapy?" The Journal of Experiential Education, 24(2): 70-79.

Saint-Onge, H. and Wallace, D. (2003). Leveraging Communities of Practice for Competitive Advantage. Butterworth- Hienemann. New York.

Sandhoff, G. (1999). "Virtual organisations as power-asymmetrical networks." Virtual Organisation Net, 1(1): 103-119.

Shane, S. (1994). "Championing innovation in the global corporation." Research Technology Management, 37(4): 29-34. 
Shultze, U. (2001). "Reflexive ethnography in information systems research." In Trauth, E. (Ed.). Qualitative Research in IS: Issues and Trends (pp. 78-103). Idea Group Publishing, London.

Siegel, P. (2000). "Using peer mentors during periods of uncertainty." Leadership \& Organisation Development Journal, 21(5): 243-253.

Smith, H. \&McKeen, J. (2003a). "Networks: Knowledge management's 'killer app'?" Queen's Centre for Knowledge-Based Enterprises 3(6): 1-13.

Smith, H. \&McKeen, J. (2003b). "Creating and Facilitating Communities of Practice." Queen's Centre for Knowledge-Based Enterprises 3(9): 1-16.

Snow, C., Miles, R. \& Coleman, H. (1992). "Managing $21^{\text {st }}$ century network organisations." Organizational Dynamics, 2(3): 5-16.

Stead, V. (2005). "Mentoring: a model for leadership development.” International Journal of Training and Development 9(3): 170-184.

Stokes, A. (2001). "Using telementoring to deliver training to SMEs: A pilot study." Education \& training, 43(6): 317-234.

Strauss, A. \& Corbin, J. (1990). Basics of Qualitative Research: Grounded Theory Procedures and Techniques. California: Sage Publications.

Swartz, E., Boaden, R. (1997). "A methodology for researching the process of information management in small firms." International Journal of Entrepreneurial Behavior \& Research, 3(1): 53-65.

Symonds, J., McCullough, G., Oliver, G. \& Brown, M. (2003). "Use of IT to support knowledge sharing: The New Zealand perspective." Proceedings of the Seventh Pacific Asia Conference on Information Systems, 10-13 July: 209-217.

Tanudidjaja, F., Kankanhalli, A. \& Tan, B. (2003). "Knowledge integration in global virtual teams." 7th Pacific Asia Conference on Information Systems, Adelaide, South Australia, 10-13 July, 2003: 1210-1223.

Thornton, G. (2008). Take a fresh look at some of the Not for Profit issues currently affecting the sector. Retrieved from Office for the Community \& Voluntary Sector, website: http:/www.ocvs.govt.nz/work-programe/building-knowledge/study-of-thenon-profit-sector/indix.html

Thornton, G. (2010). Pressing issues impacting New Zealand's Not for Profit sector. Retrieved from Office for the Community \& Voluntary Sector, website: http://www.ocvs.govt.nz/work-programe/building-knowledge/study-of-the-non-profitsector/indix.html

Tidd, J., Bessant, J. \&Pavitt, K. (1997). Managing Innovation: Integrating Technological, Organizational \& Market Change (pp.214-235). John Wiley \& Sons. 
Townsend, A., DeMarie, S. \& Hendrickson, A. (1998). "Virtual teams: Technology and the workplace of the future." Academy of Management Executive, 12(3): 17-29.

Trauth, E. (2001). "The choice of qualitative methods in IS research," in Trauth, E. (Ed.). Qualitative Research in IS: Issues and Trends (pp. 1-19). Idea Group Publishing, London.

Watson-Manheim, M. B. \& Bélanger, F. (2007). "Communication Media Repertoires: Dealing With the Multiplicity of Media Choices.” MIS Quarterly, 31(2): 267-293.

Weaver, D., Robbie, D. \& Borland, R. (2008). “The Practitioner's Model: Designing a Professional Development Program for Online Teaching." International Journal on ELearning 7(4): 759-774.

Weber, R. (2003). “The reflexive researcher.” MIS Quarterly, 27(4): v-xiv.

Weber, R. (2004). "The Rhetoric of positivism versus Interpretivism: A personal view." MIS Quarterley, 28(1): iii-xii.

Webster-Wright, A. (2009). "Reframing Professional Development Through Understanding Authentic Professional Learning." Review of Educational Research, 72(2): 702-739.

Weick, K.E. (1979a. “Cognitive processes in organization.” In B.M. Staw (Ed.). Research in organizational behavior Vol. 1 (pp. 41-74). CT: JAI Press. Greenwich.

Wenger, E. (1998). "Communities of practice: Learning as a social system." The Systems Thinker, 9(5): 1-12.

Wenger,E., McDermott, R. \& Snyder, W. (2002). Cultivating Communities of Practice: A Guide to Managing Knowledge. Harvard Business School Press. USA.

Yin, R. (1994). Case study research: Design and methods. Thousand Oaks, CA: Sage Publications

Yoong, P. and Huff, S. (2000). "Current Issues and Concerns Regarding E-commerce: An Exploratory Study of SMEs in New Zealand."Proceedings of the 2000 ETEC Conference (Track 6), Kuala Lumpur, Malaysia, November 2000: 1-5. 